\title{
Protein/peptide characterization using mass spectrometry and molecular dynamics simulations
}

\author{
Ahmad Kiani Karanji \\ West Virginia University, ak0041@mix.wvu.edu
}

Follow this and additional works at: https://researchrepository.wvu.edu/etd

Part of the Analytical Chemistry Commons, and the Biochemistry Commons

\section{Recommended Citation \\ Kiani Karanji, Ahmad, "Protein/peptide characterization using mass spectrometry and molecular dynamics simulations" (2020). Graduate Theses, Dissertations, and Problem Reports. 7953. \\ https://researchrepository.wvu.edu/etd/7953}

This Dissertation is protected by copyright and/or related rights. It has been brought to you by the The Research Repository @ WVU with permission from the rights-holder(s). You are free to use this Dissertation in any way that is permitted by the copyright and related rights legislation that applies to your use. For other uses you must obtain permission from the rights-holder(s) directly, unless additional rights are indicated by a Creative Commons license in the record and/ or on the work itself. This Dissertation has been accepted for inclusion in WVU Graduate Theses, Dissertations, and Problem Reports collection by an authorized administrator of The Research Repository @ WVU.

For more information, please contact researchrepository@mail.wvu.edu. 


\title{
Protein/peptide characterization using mass spectrometry and molecular dynamics simulations
}

\author{
Ahmad Kiani Karanji \\ Dissertation submitted to the \\ Eberly College of Arts and Sciences \\ at West Virginia University \\ in partial fulfillment of the requirements \\ for the degree of \\ Doctor of Philosophy \\ In \\ Chemistry \\ Stephen J. Valentine, Ph.D., Committee Chairperson \\ Blake Mertz, Ph.D., Committee Chairperson \\ Brian Popp, Ph.D. \\ Fabien Goulay, Ph.D. \\ Mark McLaughlin, Ph.D. \\ C. Eugene Bennett Dept. of Chemistry \\ Morgantown, West Virginia \\ 2020
}

Key Terms: protein/peptide, molecular dynamics simulation, mass spectrometry, hydrogen deuterium exchange (HDX), Huntingtin, electron transfer dissociation, Nt17, lipid vesicle, ion mobility, collision cross section, blue proteorhodopsin,

Copyright 2020 Ahmad Kiani Karanji 


\title{
Abstract \\ Protein/peptide characterization using mass spectrometry and molecular dynamics simulations
}

\begin{abstract}
Ahmad Kiani Karanji
Mass spectrometry (MS) based-techniques and molecular dynamics (MD) simulations have been used to characterize protein/peptide structure as well as their interactions with lipid vesicles and detergents. Chapter 1 introduces an introduction to the concepts and tools that were used in this work. In Chapter 2, the dominant gas-phase conformer of $[\mathrm{M}+3 \mathrm{H}]^{3+}$ ions of the model peptide Acetyl-PSSSSKSSSSKSSSSKSSSSK are examined with ion mobility spectrometry (IMS), gas-phase hydrogen deuterium exchange (HDX), and mass spectrometry (MS) techniques. This section furthers the development of a protein structural prediction tool by providing information about gas-phase ion conformers of two model peptides having different solution conformational states. In Chapter 3, interactions between the first 17 amino acid residues (Nt17 peptide) of the Huntingtin protein (htt) with lipid vesicles comprised of two subclasses of phospholipids are examined using electrospray ionization - mass spectrometry (ESI-MS) and MD simulations. Notably, the aggregation of Htt results in Huntington's disease (HD). Chapter 4 presents a MD simulations study of proteorhodopsin (PR) oligomers in different membrane environments. Proteorhodopsins are transmembrane proteins and members of the microbial rhodopsin family which act as a proton pump in the bioenergetics processes in bacteria. Finally, Chapter 5 discusses future directions for utilizing experimental and computational methods to obtain information about protein/peptide structure. Overall, the combination of MS-based techniques and MD simulations is shown to provide unique and enhanced structural information for intractable protein/peptide species.
\end{abstract}




\section{Dedication}

I dedicate this work to my lovely and caring wife, Marziyeh! I am lucky to have you as my companion; I could not go through this journey without you. And to my daughter, Melika! I want you to know that you are always my first concern. Also, to my parents Maman and Baba, your love is always inspiring for me, and my brothers Mohammad, Rasoul, and Mostafa and my sister Fatemeh. I missed all of you the entire time.

\section{Acknowledgements}

I must thank my research advisors Dr. Stephen J. Valentine and Dr. Blake Mertz for all the life and research skills that they taught me. These two men thought me how to best approach the research subjects; I applied the same critical thinking approach to the other aspects of my life. I would like to express my thanks to all my committee members: Dr. Stephen J. Valentine, Dr. Blake Mertz, Dr. Brian V. Popp, Dr. Fabian Goulay, and Dr. Mark McLaughlin for serving on my committee as well as Dr. Peng Li and Dr. Justin Legleiter for helping me. Also, I would like to thank a long list of outstanding friends and colleagues with whom I worked including: Dr. Hossein Maleki, Dr. Sadegh Faramarzi, Kushani Attanayake, Sandra N. Majuta, Daud Sharif, Anthony DeBastiani, Kinkini Jayasundara, Madison Pursell, Samira Hajian, Maryssa Beasley, Dr. Jim Arndt, Dr. Mahdiar Khakinejad, Dr. Samaneh Kondalaji, Dr. Chitrak Gupta, Nandhini Ranganathan, Chong Li, Eric Sefah, Corey Cleavenger, Violetta Burns Casamayor, Nick Frazee, and Kyle Billings. 


\section{Table of Contents}

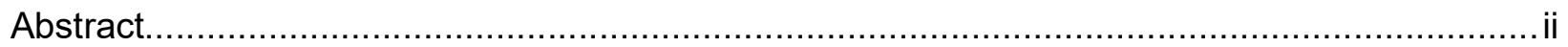

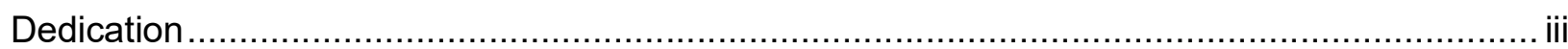

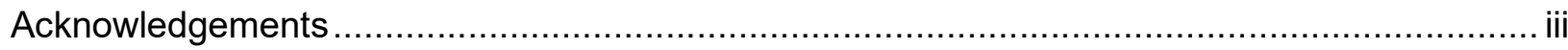

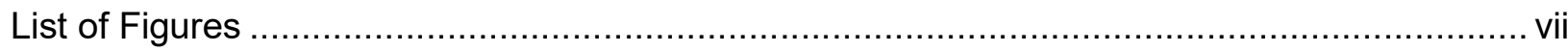

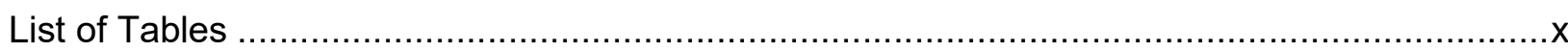

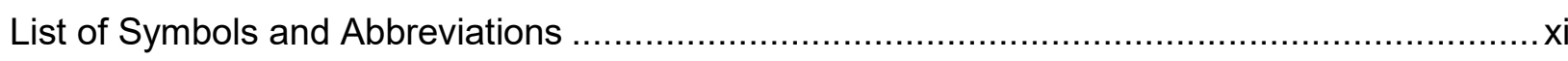

1. Introduction: Tools Used for protein/peptide structure characterization and identification of protein-lipid interactions: Electron Transfer Dissociation (CID), Ion Mobility Spectrometry-Mass Spectrometry (IMS-MS), Molecular Dynamics (MD) Simulations, and Hydrogen Deuterium

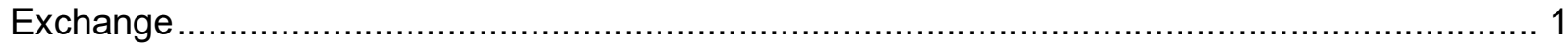

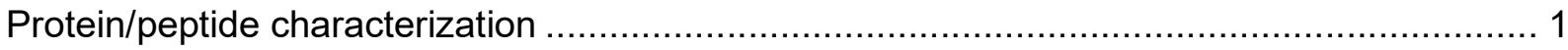

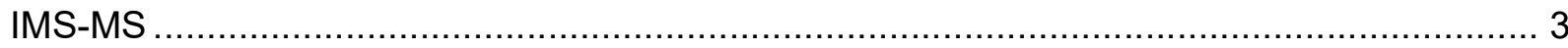

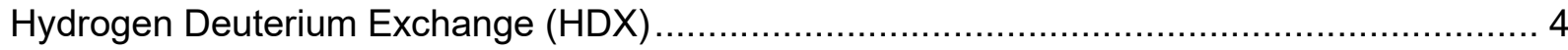

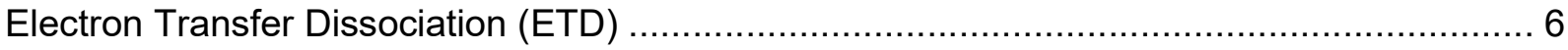

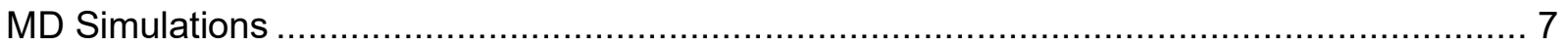

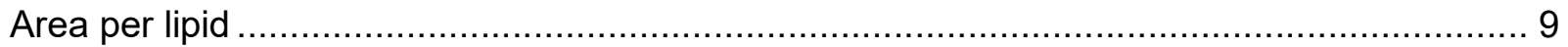

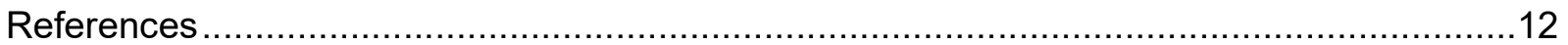

2. Comparison of Peptide Ion Conformers Arising from Non-Helical and Helical Peptides Using Ion Mobility Spectrometry and Gas-Phase Hydrogen/Deuterium Exchange ............................20

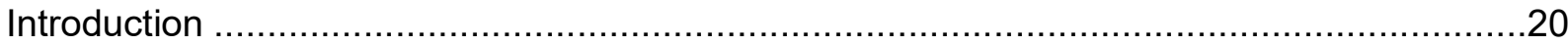

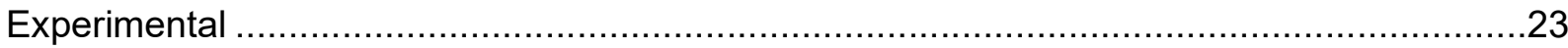

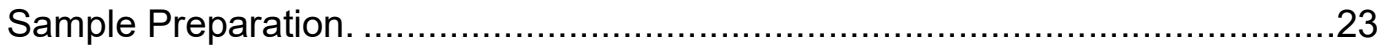

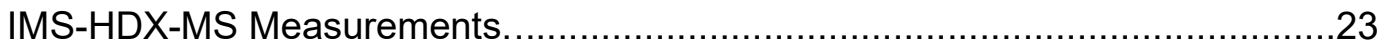

Peptide Ion Dissociation by ETD and Per-Residue Deuterium Uptake

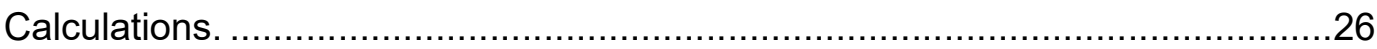

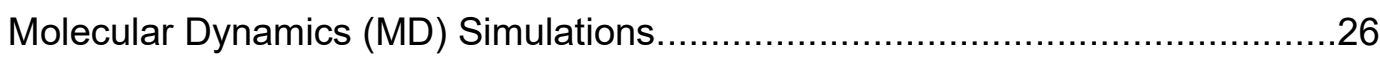

Hydrogen Accessibility Scoring (HAS) and HDX Kinetics Modeling. .................27

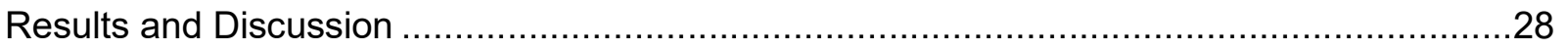

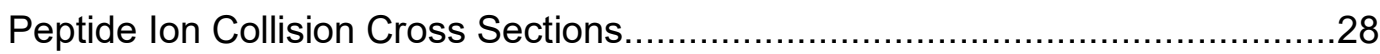

Per-residue Deuterium Uptake...............................................................

S- and A-residue deuterium content comparison............................................3

Comparison to candidate structures from MD simulations.................................36

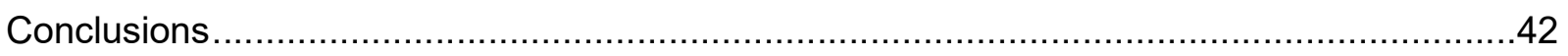

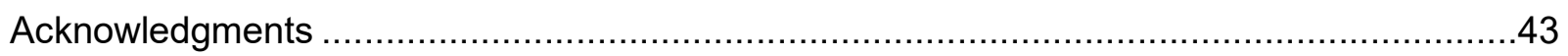


References

3. Investigating the interactions of the first 17 amino acid residues of Huntingtin with lipid vesicles using mass spectrometry and molecular dynamics .......................................48

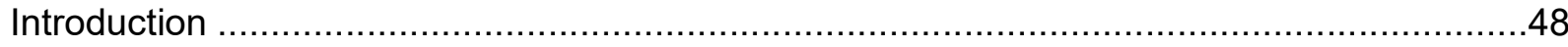

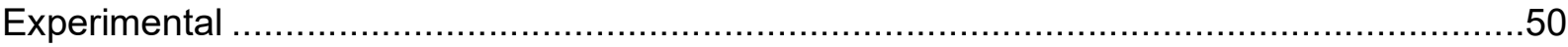

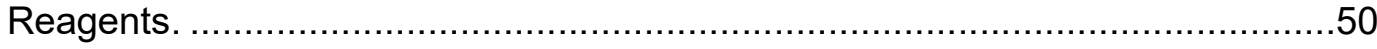

Vesicle Capture-Freeze-Drying (VCFD) . ............................................... 50

Electrospray-Mass Spectrometry (ESI-MS) ......................................... 51

Molecular Dynamics (MD) Simulations.....................................................52

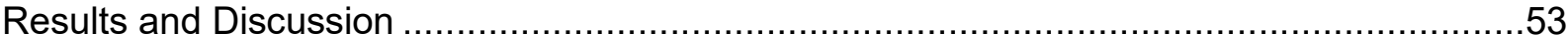

Peptide-lipid interactions examined with native MS ...................................53

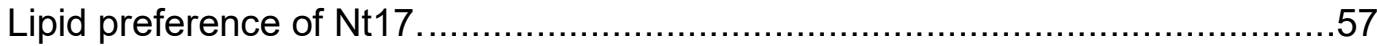

Co-mixing Nt17 peptide and lipids without vesicle formation. .......................58

Consideration of peptide-lipid ion intensities. ..........................................59

Molecular dynamics (MD) simulations: random coil interaction. .....................60

Molecular dynamics (MD) simulations: helix-membrane compatibility. ..............64

Molecular Dynamics (MD) Simulations: peptide orientation...........................67

MD simulations: potential origin of interaction differences............................71

MD simulations: peptide helical persistence........................................ 71

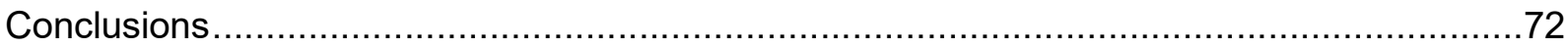

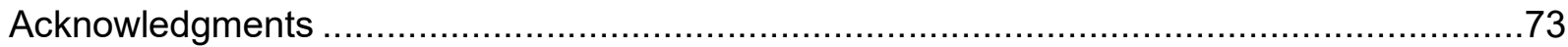

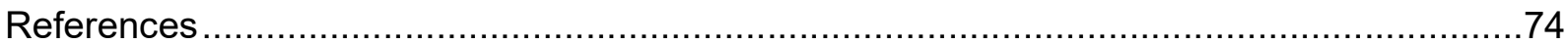

4. Molecular dynamics simulations of Proteorhodopsin oligomers in lipid bilayer and micellar

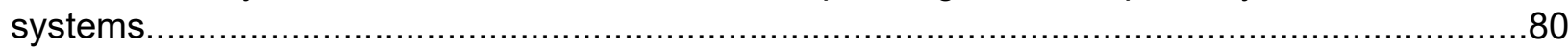

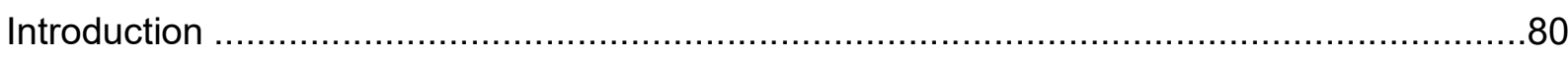

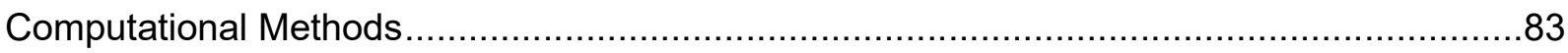

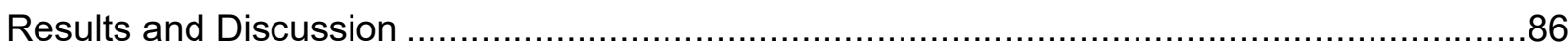

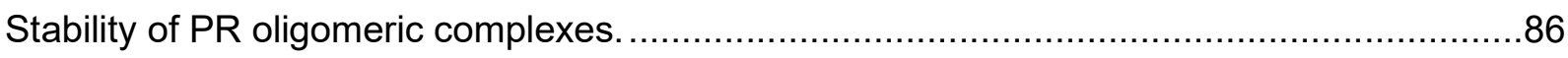

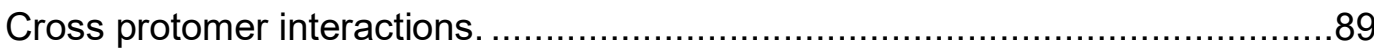

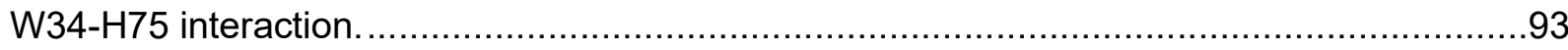

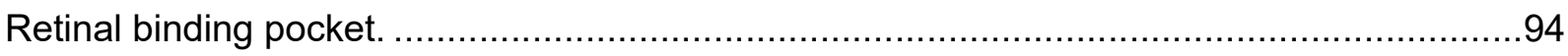

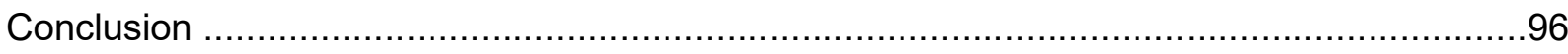

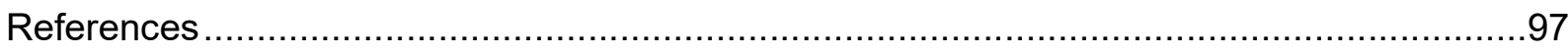

5. Future Directions: protein characterization using mass spectrometry and molecular dynamics simulations. 


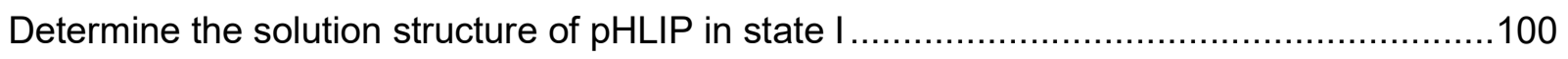

Determine the effect of membrane composition on permeation of pHLIP in membrane.......103

The strange case of leptin; an electrospray ionization study .....................................106

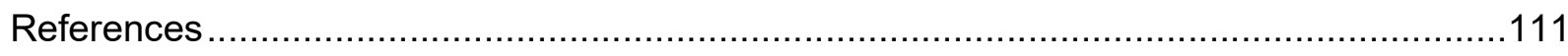

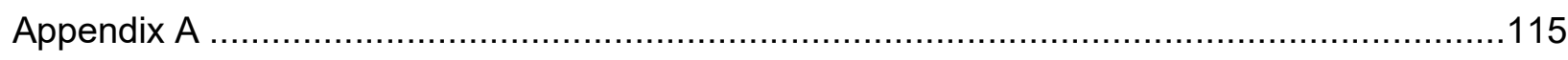

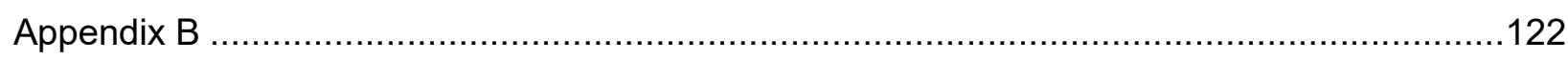

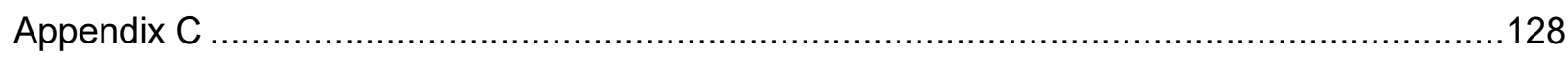




\section{List of Figures}

Figure 1. 1 Schematic diagram of an IMS-MS instrument used in the study of protein and peptide ion structures. Shown is the electrospray ionization source, the source desolvation region, the ion funnel $(F 1)$, ion activation $(I A)$, and ion gate $(G)$ regions. Also shown are the drift tube lenses. Finally, the linear ion trap mass spectrometer is also shown. Figure adapted with permission from ${ }^{70}$.

Figure 1. 2 Area per lipid Phenomenon. Shown are the lipid structures (left) for DMP, DOPC, and PhPC. The bilayer formed by each lipid system is illustrated in front of each lipid. The areaper-lipid values for DMPC, DOPC and PhPC are 59.0 A22 [93], $67.4 \AA 2$ [94], and $80.6 \AA 2$ [20] respectively at $30^{\circ} \mathrm{C}$.

Figure 2. $1 \mathrm{t}_{\mathrm{D}}$ distributions for the $[\mathrm{M}+3 \mathrm{H}]^{3+}$ ions of the model peptide AcetylPSSSSKSSSSKSSSSKSSSSK 25

Figure 2. 2 lon fragmentation spectra obtained upon ETD of the $[\mathrm{M}+3 \mathrm{H}] 3+$ ions of the model peptide Acetyl-PSSSSKSSSSKSSSSKSSSSK.

Figure 2. 3 Bar graph showing the fraction of deuterium for $S$ (red bars) and A (blue bars) residues obtained from $\mathrm{MS} / \mathrm{MS}$ analysis of mobility selected ions.

Figure 2. 4 The comparison of the experimental and theoretical fraction deuterium incorporated values.

Figure 3. 1 Full range mass spectrum of the peptide-DOPC system.

Figure 3. 2 Area per lipid calculations of the 100-member lipid bilayer for the DOPC, POPC and POPE and relative helical propensity for Nt17 peptide in the presence of these lipid vesicles...61 Figure 3. 3 Schematic representations of random coil Nt17 peptide interacting with the DOPC bilayer.....

Figure 3. 4 Heat maps showing the distance between $\alpha$-carbons of each residue on the Nt17 peptide to the center of the bilayer as a function of MD simulation time. .66 Figure 3. 5 Molecular representations of the position/orientation of the Nt17 peptide on the surface of the DOPC lipid bilayer, and the POPE lipid bilayer.

Figure 4. 1 Proteorhodopsin photocycle. 81

Figure 4. 2 Pentameric form of Proteorhodopsin. ........................................................................... 82

Figure 4. 3 Packing score as measured for all oligomeric systems. ........................................87

Figure 4. 4 Monomer-monomer distances between helix bundles: lipid bilayer vs. detergents. 89 Figure 4. 5 Salt bridge distances between E50-R51 ................................................................ 90

Figure 4. 6 Salt bridge distances between D52-R51 .............................................................. 92

Figure 4. 7 Salt bridge distances between D52-K59 ............................................................... 93

Figure 4. 8 Hydrogen bond between W34 and H75 .............................................................94

Figure 4. 9 Retinal binding pocket: Schiff base to D227 ............................................................ 95 
Figure 5. $1 \mathrm{CD}$ spectrum of pHLIP recorded at two different $\mathrm{pH}$ levels.

Figure 5. 2 Online hydrogen deuterium exchange apparatus. The peptide solution and water mixing occurs at T1 and HDX occurs along L. By changing this length, different exchange rates can be measured.

Figure 5. 3 The mass spectrum (ESI-MS) for a pHLIP-POPC system. Different monomeric and multimeric ions are labeled (see legend for details.

Figure 5. 4 Mass spectra for for A) Myoglobin and B) leptin generated by native MS. Here the proteins were electrosprayed from buffered (100 mM ammonium acetate) solutions. Charge states are labeled for each protein. Insets show the structures of the two different proteins. Note the long, unstructured region for the protein leptin which comprises an entire face of the protein.

Figure 5. 5 The distance profile of the center of mass for myoglobin and leptin proteins to the water droplet center of mass. The distance to the droplet surface is provided. The two different traces are labeled for the respective proteins.

Figure S2. 1 CD spectroscopy of both model peptides PAAAAKAAAAKAAAAKAAAAK and PSSSSKSSSSKSSSSKSSSSK.

Figure S2. 2 Conformational space sampling of 40-ps and 1200-ps SA for each conformer arrangement.

Figure S2. 3 An example of very compact ion conformer type with charge configuration of K6_K11_K21 and theoretical CCS value of 348 Å2 Calculated by Mobcal software.

Figure S2. 4 The increase in the predicted deuterium content for the conformer with high accessibility at the terminal peptide portions rather than the interior residues.

Figure S2. 5 An example of a structure with a wrong CCS value but showing a high D incorporation value for S20.

Figure S3. 1 Mass spectrum showing the ions obtained upon ESI of the Nt17-POPC and Nt17POPE vesicle systems.

Figure S3. 2 Relative intensities of lipid-bound peptide peaks in mass spectra for Nt17-lipid vesicles.

Figure S3. 3 Isolation of $[\mathrm{Nt} 17+1 \mathrm{~L}+2 \mathrm{H}] 2+$ ions for DOPC $(\mathrm{A})$ and POPE $(B)$ in the linear ion trap mass spectrometer.

Figure S3. 4 The relative degree of peptide helicity as a function of simulation time for the three lipid systems.

Figure S4. 1 R51 dihedral angle in DHPC-H. A) D52-R51 distance, B) E50-R51 distance. 128 Figure S4. 2 R51 dihedral angle in DHPC-P. A) D52-R51 distance, B) E50-R51 distance. 129 
Figure S4. 3 Packing score for WT vs mutations.

Figure S4. 4 Distance between the center of monomers, WT vs mutations. 130

Figure S4. 5 Distance between $\mathrm{H} 75$ and D97.

Figure S4. 6 H75 dihedral angle. A) H75-D97 B) W34-H75 distances in LB-Pentamer system.

Figure S4. 7 H75 dihedral angle. A) H75-D97 B) W34-H75 distances in LB-Hexamer system. 


\section{List of Tables}

Table 3. 1 Assigned compounds and their corresponding mass to charge ratio 56

Table 4. 1 Simulation method and run times for all membrane systems.

.85

Table S2. 1 Deuterium uptake values calculation for serine residues in the model peptide.....121

Table S3. 1 Structural properties of the modeled lipid membranes. 


\section{List of Symbols and Abbreviations}

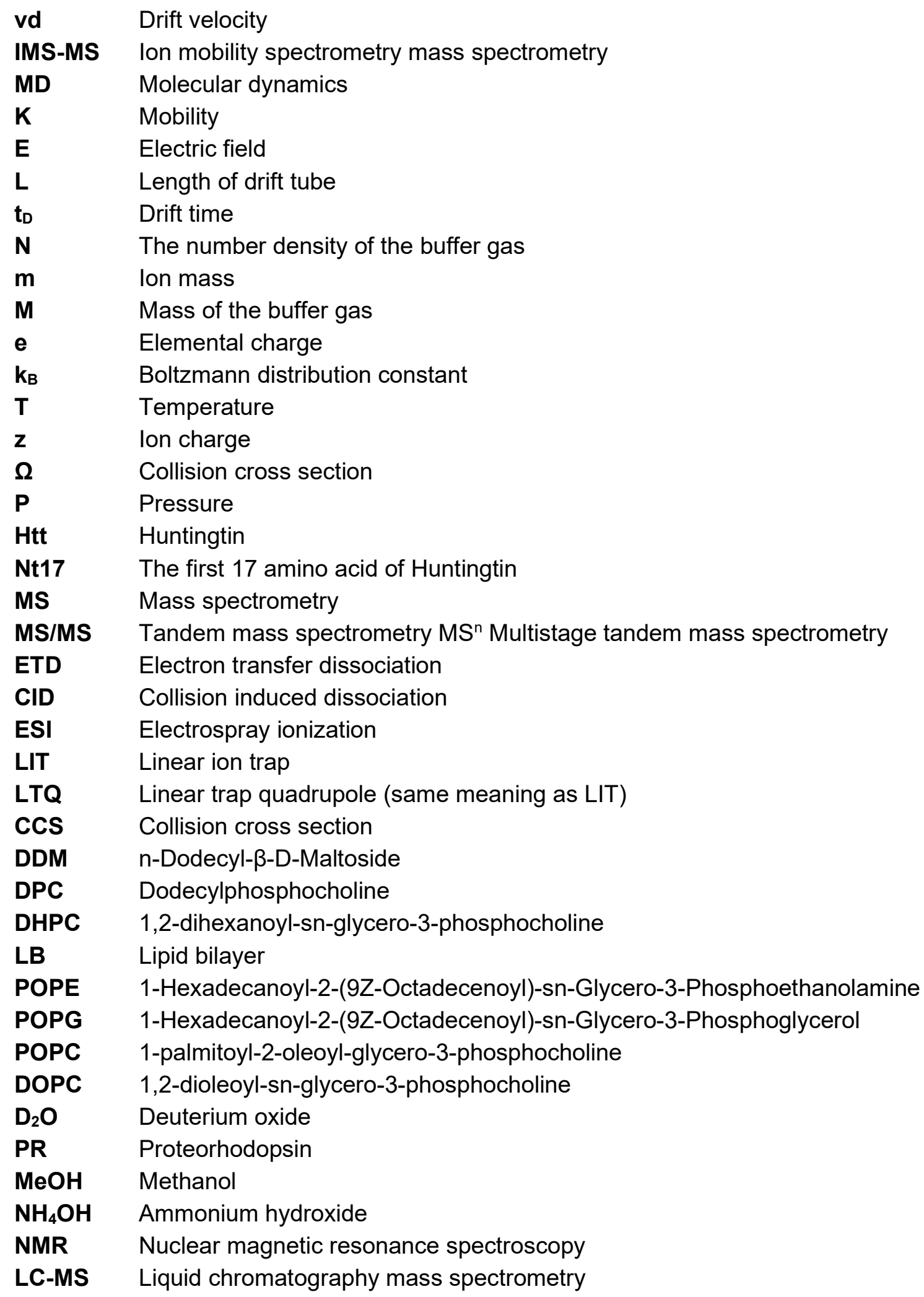




$\begin{array}{ll}\text { GC } & \text { Gas chromatography } \\ \text { GC-MS } & \text { Mass spectrometry-Gas chromatography } \\ \text { LC } & \text { Liquid chromatography } \\ \text { RMSD } & \text { Root mean square of the deviation } \\ \text { HAS } & \text { Hydrogen accessibility scoring } \\ \text { PDB } & \text { Protein data bank } \\ \text { VMD } & \text { Visual Molecular Dynamics } \\ \text { NAMD } & \text { Nanoscale Molecular Dynamics }\end{array}$




\section{Introduction: Tools Used for protein/peptide structure characterization and identification of protein-lipid interactions: Electron Transfer Dissociation (CID), lon Mobility Spectrometry-Mass Spectrometry (IMS-MS), Molecular Dynamics (MD) Simulations, and Hydrogen Deuterium Exchange}

\section{Protein/peptide characterization}

Proteins are biopolymers containing sequences of amino acids bound together by peptide bonds. These large biomolecules are involved in many biological activities such as energy conversions, and chemical transportation ${ }^{1-3}$. The 3D structural characterization of proteins is an important pre-requisite for studying the function of these large biomolecules in biological media ${ }^{4-5}$. An error in the protein structure may lead to disease; for example; misfolding of Huntingtin protein (htt) can lead to aggregation and the formation of amyloid fibrils in neurons ${ }^{6}$.

Historically, protein characterization has been conducted with spectroscopic methods such as nuclear magnetic resonance (NMR) ${ }^{7-14}$, fluorescence ${ }^{15-18}$ and circular dichroism (CD) 12, 19-20, UV-based methods ${ }^{21-22}$ and Raman spectroscopy ${ }^{23}$. Along with these powerful analytical techniques high resolution methods such as x-ray crystallography ${ }^{24-26}$ and cryoelectron microscopy ${ }^{27-30}$ can be used to study protein structure and their interactions. Although these traditional techniques are widely used, they suffer from some limitations such as difficulties in sample preparation ${ }^{31}$. It is worth mentioning that there is a limitation in crystalizing some of the proteins ${ }^{32}$. In addition, many of the membrane proteins are not soluble in water because of their hydrophobic nature; therefore it can be difficult to study such structures using NMR ${ }^{9}$. Furthermore, some globular proteins tend to aggregate and create big systems which are difficult to study using NMR ( $15 \mathrm{KDa}$ limitation $){ }^{31}$. Another limitation can be that some of 
the protein interactions are transient and cannot be captured using these traditional techniques 33. Finally, it should be noted that an increasing number of studies is beginning to show the presence of multiple, coexisting structures under native conditions ${ }^{34-36}$. Some of these limitations can be addressed with mass spectrometry (MS) and mass spectrometry-based techniques such as hydrogen deuterium exchange (HDX). Several advantages include those associated with analysis speed and sensitivity as well as an ability to "freeze out" coexisting structures ${ }^{37-40}$.

Early MS-based studies of proteins involved studies of the conformational properties and the folding pathways of native-like proteins using electrospray ionization-mass spectrometry (ESI-MS) ${ }^{41-45}$. These studies were often performed to obtain information about the mechanism of action of these important biomolecules. Shortly later, gas-phase separation techniques were introduced as a tool to characterize protein structure ${ }^{46-49}$. In the gas phase, protein ion structure is not stabilized through hydrogen bonding with the solvent molecules $46,50-51$. Ion mobility spectrometry-mass spectrometry (IMS-MS) was introduced to effectively increase the resolution of the MS data and reveal different gas-phase structures corresponding to co-existing protein conformations in solution ${ }^{48,52}$. For gas-phase ion structure characterization, IMS is an appropriate separation technique because of its high compatibility with mass spectrometry measurements ${ }^{53-56}$. Indeed, IMS-MS measurements were used early on to suggest that many protein ions could remain in their solution conformational state for hundreds of milliseconds which is sufficiently long enough to study with ion mobility instruments ${ }^{57-59}$. Other aspects of MS-based studies include the theoretical investigations of the ionization processes of protein ${ }^{60-}$ 61, and labeling techniques ${ }^{31,37,62-63}$.

The combination of experimental techniques such as MS with molecular dynamics (MD) simulations can provide valuable information regarding protein structures as well as their 
interactions with other chemical species ${ }^{64-67}$. For example, MS can be used to provide information about the contact sites of a protein interacting with other proteins or with other biological compounds, as well as finding the structured/unstructured and rigid/dynamic regions of a protein followed by relating this information to the higher-order structure ${ }^{31}$. The atomistic view of MD simulations enables scientists to have a better understanding of the chemistry of these molecules.

\section{IMS-MS}

Ion mobility spectrometry (IMS) is a gas-phase ion separation technique that has frequently been used to study the structure of biomolecular ions ${ }^{49}$. The high compatibility of this separation technique with MS makes it available in many newly designed commercial instrumentations ${ }^{68-69}$. In this technique the separation is realized according to the ion's charge and overall size. IMS instrumentation consists of a drift tube (a pressurized enclosure) with electrostatic lenses (Figure 1.1) ${ }^{70}$. The first step in an IMS measurement scheme is to ionize the molecules thereby transferring the solution-phase molecules to the gas phase as ions. To detect the molecules using a mass analyzer requires that the molecules are charged. Therefore, an ionization technique to be used in such experiments should provide these two requirements (volatilization and ionization) ${ }^{60}$. The ions originating from the source are then introduced to a drift tube such as that shown in Figure 1.1 and subsequently separated according to their sizeto-charge ratios. The drift tube can be coupled with different mass analyzers such as the LTQ mass spectrometer for mass analysis and ion detection ${ }^{71}$. 


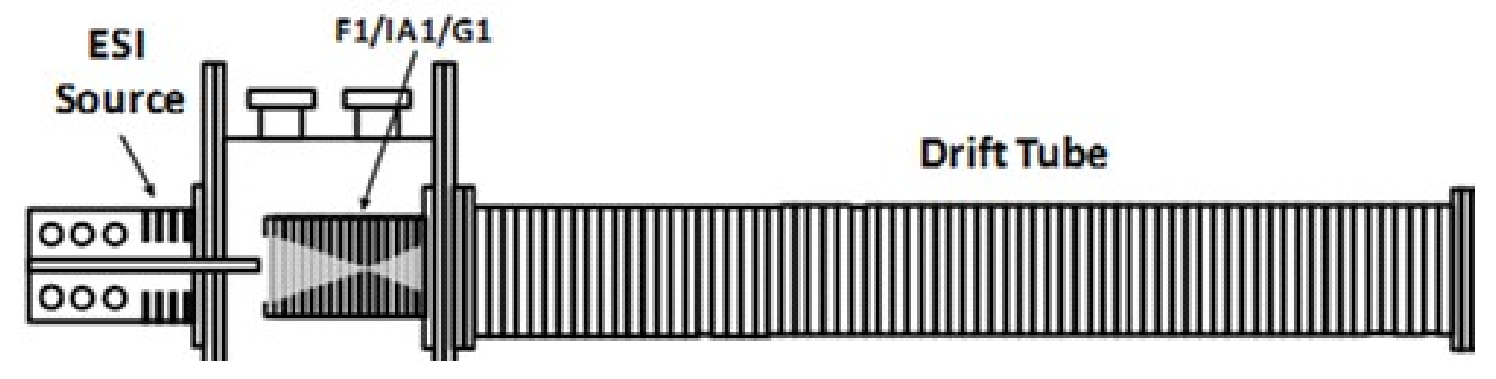

Figure 1. 1 Schematic diagram of an IMS-MS instrument used in the study of protein and peptide ion structures. Shown is the electrospray ionization source, the source desolvation region, the ion funnel $(\mathrm{F} 1)$, ion activation $(\mathrm{IA})$, and ion gate $(\mathrm{G})$ regions. Also shown are the drift tube lenses. Finally, the linear ion trap mass spectrometer is also shown. Figure adapted with permission from ${ }^{70}$.

An ion's transit time inside the drift tube is referred to as the drift time $\left(t_{D}\right)$. This tD value can be used to calculate the collision cross-section (CCS) of the ion ${ }^{72}$. The CCS can be loosely described as the projection of the orientationally-averaged ion or the average area swept through the buffer gas. CCS values can be calculated using the equation below.

$$
\Omega=\frac{(18 \pi)^{1 / 2}}{16} \frac{z e}{\left(k_{B} T\right)^{1 / 2}}\left[\frac{1}{m_{I}}+\frac{1}{m_{B}}\right]^{1 / 2} \frac{t_{D} E}{L} \frac{760}{P} \frac{T}{273.2} \frac{1}{N} \quad \text { Equation } 1.1
$$

In Equation 1.1, $k_{B}$ is the Boltzmann's constant, $T$ is the buffer gas temperature, the term $\left[\frac{1}{m_{I}}+\right.$ $\left.\frac{1}{m_{B}}\right]$ is the reduced mass, $E$ is the electric field, and $L$ is the length of the drift tube.

\section{Hydrogen Deuterium Exchange (HDX)}

One of the commonly used MS-based techniques in the study of protein structure is that of hydrogen deuterium exchange (HDX). Traditionally performed in solution, HDX is based on the replacement of accessible amide hydrogens with deuteriums along the protein backbone ${ }^{1}$, 73-74. Areas in which exchange does not occur can then be ascertained using MS measurements and such areas can be suggested to be involved in important interactions such as the hydrogen bonding networks associated with secondary structural elements (a helices and b sheets) ${ }^{63,75-79}$. HDX experiments are possible because accessible protons on the protein are 
constantly exchanging with the solvent protons; this process is not detectable by MS because no mass change occurs ${ }^{1}$. To carry out HDX experiments the sample is reacted with a deuterated solvent such as $\mathrm{D}_{2} \mathrm{O}$. Depending on the structure of the protein, some of the backbone amide hydrogens will exchange with deuteriums. The HDX reaction can be quenched after a certain period of time by lowering the $\mathrm{pH}$ and temperature ${ }^{80}$. Factors that affect the amount of deuterium incorporation include the solution temperature and $\mathrm{pH}$ and the tertiary structure of the protein ${ }^{81}$. Lowering the $\mathrm{pH}$ to a point $(\sim 3.0){ }^{80}$ will result in decreased HDX rates. Thereafter, HDX rates increase and deuterium incorporation is increased. Complete HDX can happen on the ms time scale for random coil portions of proteins, but it will take minutes to hours for the parts of the protein involved in establishing secondary structure. Therefore, protection information can be correlated to higher order structure of proteins ${ }^{81}$.

Different methods can be utilized to detect the incorporated deuteriums in the protein structure. One approach is to digest the protein to obtain smaller peptides using an enzyme in the solution phase. This is followed by cold liquid chromatography (LC) to separate the peptides and detect them using subsequent mass analysis ${ }^{82-85}$. Another approach is to utilize the intact protein ions and fragment them in the gas phase to determine where the deuteriums were incorporated. In either case, the gas-phase dissociation technique to be used should be non-ergodic in order to avoid the mobilization and scrambling of the deuterium label ${ }^{86-88}$.

More recently, gas-phase HDX has been introduced as a means to elucidate protein/peptide ion structure $62-63,89-91$. Here, the gas-phase protein ions are subjected to collisions with a deuterating reagent gas (typically ND3 and D2O). As with solutionphase experiments, reaction occurs for accessible hydrogens. A notable difference is that accessible hydrogens are those that are not buried (accessible to collisions) or are accessible to original incorporation sites $1,75,78$. Therefore, the determination of 
incorporation site is directly related to structural features such as surface accessibility as well as accessibility to charge sites. These features have been successfully modeled to provide an estimation of the overall ion structure ${ }^{92-93}$.

\section{Electron Transfer Dissociation (ETD)}

As mentioned above, HDX is a powerful method to study the structures of biomolecules. Additionally, HDX can be performed in the solution phase using liquid chromatography-mass spectrometry (LC-MS) ${ }^{94}$ and online HDX ${ }^{95}$, or in the gas phase using drift tube ion mobility spectrometry ${ }^{96}[$ more refs including Clemmer]. There are two main methods of protein characterization in the fields of structural and comparative proteomics. Introducing the peptides resulting from enzymatic cleavage in the solution phase to the mass spectrometer is known as the bottom-up approach ${ }^{79-80}$. Conversely, transferring the intact protein into the mass spectrometer and using an appropriate gas-phase dissociation method is known as the topdown approach ${ }^{87-88}$. The former approach is the most commonly used method to detect deuterium exchange in proteins, but it suffers from some limitations. One of the limitations for bottom-up HDX is the deuterium back exchange with hydrogens ${ }^{97}$. This can occur to some extent during the separation step and for this reason the HPLC separation is maintained at cold temperatures. Additionally, it can occur in the gas phase before the de-solvated ions are analyzed by the mass spectrometer. Another limitation can be the lack of complete sequence coverage using a bottom-up approach for some proteins ${ }^{98-99}$. To overcome these limitations, the top-down approach can be utilized to determine the sites of deuterium incorporation. Top-down

proteomics can result in a per-residue level of resolution ${ }^{100}$. That said, there are only few dissociation methods that can be used effectively in the top-down approach. The main problem with conventional gas-phase fragmentation methods like collision-Induced dissociation (CID) and higher-energy collisional dissociation (HCD) is that these methods elevate the internal 
energy of the biomolecular ions ${ }^{1}$. This excess amount of energy increases the mobilization of exchangeable hydrogens which cases the protons (and deuterons) to move across the peptide backbone and to other heteroatom sites. In the case of HDX this will randomize the distribution of deuterium across the biomolecule. This process is called HDX scrambling ${ }^{94}$. Strategies to overcome this problem are to use faster fragmentation techniques such as electron transfer dissociation (ETD) ${ }^{101-103}$. Here, fragmentation happens after the molecule reacts with the ETD reagent ions (typically radical anions). For ETD, a highly-charged ion (triply charged or quadropoly charged) is often required.

\section{Simulations}

Molecular dynamics (MD) simulations is based on Newton's second law ${ }^{104}$. Basically, it is possible to calculate the position of the system at $t+\Delta t$ and write a trajectory for the system using Newton's second law of motion (Equation 1.3) when the acceleration of the system, the initial position at time $t$, and the position at time $t-\Delta t$ are available as demonstrated in Equation 1.2 and 1.3 .

$$
\begin{array}{cr}
r(t+\delta t) \approx 2 r(t)-r(t-\delta t)+a(t) \delta t^{2} & \text { Equation 1.2 } \\
m_{i} \frac{d^{2} \vec{r}_{i}}{d t^{2}}=\vec{F}_{i}=-\vec{\nabla} U(\vec{R}) & \text { Equation 1.3 }
\end{array}
$$

In these equations, $m_{i}$ is the mass, and $\vec{r}_{i}$ is the position for each atom and $\vec{F}_{i}$ is the applied force on that atom. Also $U(\vec{R})$ is the potential energy which is defined as:

$$
U\left(\vec{r}_{1}, \vec{r}_{2}, \vec{r}_{3}, \ldots \vec{r}_{N}\right)=U(\vec{R}) \quad \text { Equation } 1.4
$$

To write a trajectory it is essential to calculate the acceleration of an atom. Using equation 1.3 the acceleration (a) of each atom can be calculated. In order to calculate $a$, a knowledge of the forces exerted upon it is required. And finally, to acquire the forces, it is necessary to define energy functions $U\left(\vec{r}_{1}\right)$ for each particle. These energy functions are the sum of bonded and nonbonded terms. The bonded terms are usually defined for bonds 
(Equation 1.5), angles, dihedrals angles, and improper angles (Equation 1.6). Non-bonded interactions consist of Lenard-Jones potentials and charge-charge potentials (Equation 1.7).

$$
\begin{array}{ll}
U(\vec{R})=U_{\text {bonded }}+U_{\text {nonbonded }} & \text { Equation 1.5 } \\
U_{\text {bonded }}=U_{\text {bond }}+U_{\text {angle }}+U_{\text {dihedral }}+U_{\text {improper }} & \text { Equation 1.6 } \\
U_{\text {nonbonded }}=U_{\text {Vander Waals }}+U_{\text {Electrostatics }} & \text { Equation 1.7 }
\end{array}
$$

For each of the $U_{\text {bonded }}$ terms the definition is:

$$
\begin{array}{ll}
U_{\text {bond }}=\sum k_{i}^{\text {bond }}\left(r_{i}-r_{0}\right)^{2} & \text { Equation 1. 8, } \\
U_{\text {angle }}=\sum k_{i}^{\text {angle }}\left(\theta_{i}-\theta_{0}\right)^{2} & \text { Equation 1. 9, and } \\
U_{\text {dihedral }}=\sum k_{i}^{\text {dihedral }}\left[1+\cos \left(n_{i} \emptyset_{i}+\delta_{i}\right)\right] & \text { Equation 1. 10, }
\end{array}
$$

in which $k_{i}^{b o n d}$ is the bond energy constant, $r_{i}$ is the distance between any pair of atoms, $r_{0}$ is the equilibrium distance between any pair of atoms, $k_{i}^{\text {angle }}$ is the angular energy constant, $\theta_{i}$ is the angle between three atoms bond together, $\theta_{0}$ is the equilibrium angle between three atoms bond together, $k_{i}^{\text {dihedral }}$ dihedral energy constant, $n_{i}$ is multiplicity, $\emptyset_{i}$ is the dihedral angle between 4 atoms, and $\delta_{i}$ is the angle where the potential passes through its minimum value.

For the $U_{\text {nonbonded }}$ terms the definition is:

$$
\begin{array}{lr}
U_{\text {Vander Waals }}=\sum_{i=1} \sum_{j \neq i} 4 \varepsilon_{i j}\left[\left(\frac{\sigma_{i j}}{r_{i j}}\right)^{12}-\left(\frac{\sigma_{i j}}{r_{i j}}\right)^{6}\right] & \text { Equation 1. 11, and } \\
U_{\text {Electrostatics }}=\sum_{i=1} \sum_{j \neq i} \frac{q_{i} q_{i}}{\varepsilon r_{i j}} & \text { Equation 1. 12, }
\end{array}
$$

in which $\varepsilon_{i j}$ is the depth of the potential well, $\sigma_{i j}$ is the finite distance at which the inter-atomic potential is zero, $r_{i j}$ is the distance between two atoms, $q_{i}$ and $q_{j}$ are atomic charges, and $\varepsilon$ is Coulomb's constant.

One of the applications of MD simulations in the field of mass spectrometry is to study the behavior of molecules during the final stages of the ESI process ${ }^{60}$. An advantage of this application arises because of the time scale over which the MD simulations can now be 
performed as well as the fact that they provide an atomistic view that cannot be obtained in any other fashion. This is the subject of Chapter 5 in this report.

\section{Area per lipid}

One of the concepts that is widely used in this document is the change of lipid composition along with the tracking of the interaction of peptides with such membrane systems. The lipid composition in eukaryotic membranes consists primarily of phosphatidylcholine (PC) 105. This lipid type occupies more than $50 \%$ of the phospholipids in almost all eukaryotic membranes. In an aqueous environment, phosphatidylcholine self-organizes spontaneously and creates a lipid bilayer in which the polar headgroups face the polar solution and the nonpolar tails face each other. Some of the PC lipids are saturated and therefore their tails are straighter. A saturated PC has a low area-per-lipid value because of this ordered structure (Figure 2). DMPC is one of the PC lipids that has a low area-per-lipid value because of the completely saturated tail. Most PC lipids have one or two cis-unsaturated fatty acyl chain(s). This causes an increase in the area-per-lipid value for the bilayer. Another way to increase the area-per-lipid value is to introduce methyl groups in the tail such as 1,2-di-O-phytanyl-sn-glycero-3phosphocholine (PhPC). Figure 2 summarizes the area per lipid phenomenon.

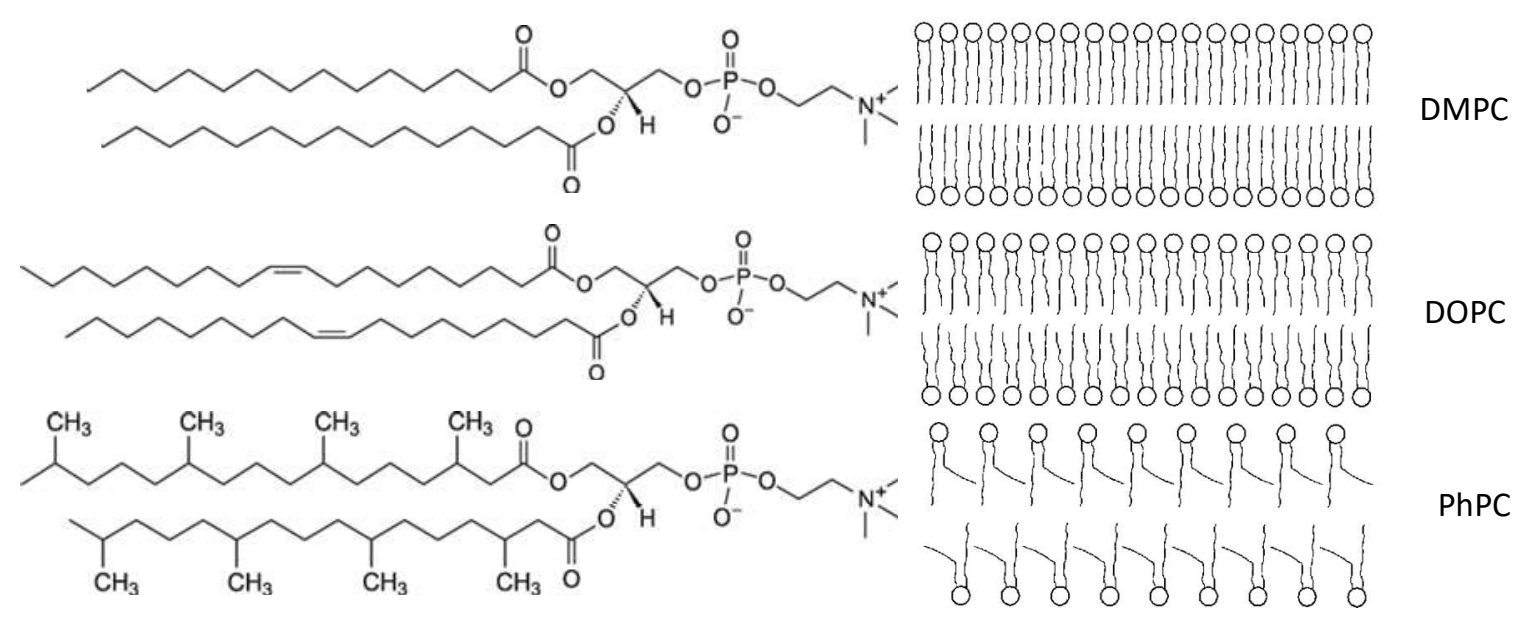


Figure 1. 2 Area per lipid Phenomenon. Shown are the lipid structures (left) for DMPC, DOPC, and PhPC. The bilayer formed by each lipid system is illustrated in front of each lipid. The area-per-lipid values for DMPC, DOPC and PhPC are $59.0 \AA 2$ [93], $67.4 \AA 2$ [94], and $80.6 \AA 2$ [20] respectively at $30^{\circ} \mathrm{C}$.

\section{Structure Studies}

In Chapter 2 a comparison between two synthetic peptides that differ significantly in their native structure in the solution phase is performed using IMS, HDX, and MD simulations. For a serine containing peptide (Acetyl-PSSSSKSSSSKSSSSKSSSSK), the $[\mathrm{M}+3 \mathrm{H}]^{3+}$ ions are observed predominantly as a relatively compact conformer type. Upon subjecting these ions to electron transfer dissociation (ETD), the level of protection for each amino acid residue in the peptide sequence has been assessed. The overall per-residue deuterium uptake is observed to be relatively more efficient for the neutral residues than for the model peptide Acetyl-PAAAAKAAAAKAAAAKAAAAK. In comparison, the N-terminal and C-terminal regions of the serine peptide show greater relative protection compared with interior residues. Molecular dynamics (MD) simulations are used to generate candidate structures for collision cross section and HDX reactivity matching. Hydrogen accessibility scoring (HAS) for select structural candidates from MD simulations is used to suggest conformer types that could contribute to the observed HDX patterns. The results are discussed with respect to recent studies employing extensive MD simulations of gasphase structure establishment of a peptide system.

In Chapter 3 of this report, the interactions of the first 17 amino acid residues (Nt17 peptide) of the Huntingtin protein (htt) with lipid vesicles is investigated ${ }^{106}$. Huntington's disease $(\mathrm{HD})$ is a neurodegenerative disease ${ }^{3,107}$ caused by the expansion of a polyglutamine-coding CAG repeat in the HTT gene ${ }^{108-112}$. The first 17 amino acid residues of htt (Nt17 of htt) are thought to play an important role in the protein's function; $\mathrm{Nt17}$ is one of two membrane-binding domains in htt. The Nt17 peptide is intrinsically disordered in 
solution and it undergoes a conformational shift from unstructured to an amphipathic $\alpha$ helix in the presence of a membrane ${ }^{6,111,113-114}$. This conformational change makes the Nt17 peptide a lipid-binding domain for $\mathrm{htt}{ }^{115}$. It is believed that the amphipathic characteristic of the peptide causes the more hydrophilic residues to face toward the polar solvent and the more hydrophobic residues to face toward the lipid vesicles ${ }^{116}$. The composition of the lipid vesicle plays an important role in this interaction as the peptide

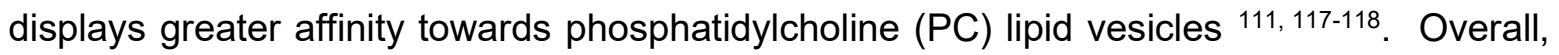
the peptide is shown to have a greater propensity to interact with vesicles of phosphatidylcholine $(\mathrm{PC})$ rather than phosphatidylethanolamine (PE) lipids. Mass spectra show an increase in lipid-bound peptide adducts where the ordering of the number of such specie is 1,2-dioleoyl-sn-glycero-3-phosphocholine (DOPC) > 1-palmitoyl-2-oleoyl-glycero3-phosphocholine (POPC) > 1-palmitoyl-2-oleoyl-sn-glycero-3 phosphoethanolamine (POPE). MD simulations suggest that the compactness of the bilayer plays a role in governing peptide interactions. The peptide shows greater disruption of the DOPC bilayer order at the surface to interact with the hydrophobic tails of the lipid molecules via hydrophobic residues. Conversely, the POPE vesicle remains ordered and lipids display transient interactions with the peptide through the formation of hydrogen bonds with hydrophilic residues. The POPC system displays intermediate behavior regarding the degree of peptide-membrane interaction. Finally, the simulations suggest a helix stabilizing effect resulting from the interactions between hydrophobic residues and the lipid tails of the DOPC bilayer.

In Chapter 4, the stability and the role of important residues in oligomeric forms of PR in a POPE:POPG lipid bilayer and different detergent systems is investigated. Several residues play a role in the ion pumping process from the cytoplasmic side to the extracellular side including E108 which acts as a proton donor, the retinol, D97 which acts 
as a proton acceptor, and E142 which is the proton releaser. In the dark state where no photon is illuminated, all these residues are in their ground state ${ }^{119-120}$. It has been suggested that the native state of the protein is a pentameric/hexametric form because these oligomeric forms are dominant for many detergent/lipid conditions. The protein obtains structural stability upon forming noncovalent interactions between the monomers in the oligomeric form. In this work molecular dynamics (MD) simulations are utilized to characterize the effect of different membrane environments on the stability of PR in the dark state. Dodecylphosphocholine (DPC), 1,2-dihexanoyl-sn-glycero-3-phosphocholine (DHPC), and n-Dodecyl- $\beta$-D-Maltoside (DDM) detergent systems along with 1Hexadecanoyl-2-(9Z-Octadecenoyl)-sn-Glycero-3-Phosphoethanolamine (POPE) and 1Hexadecanoyl-2-(9Z-Octadecenoyl)-sn-Glycero-3-Phosphoglycerol (POPG) lipid bilayer systems are used to study the pentameric/hexameric forms of PR.

\section{References}

1. Konermann, L.; Pan, J.; Liu, Y. H., Hydrogen exchange mass spectrometry for studying protein structure and dynamics. Chem Soc Rev 2011, 40 (3), 1224-34.

2. Masson, G. R.; Burke, J. E.; Ahn, N. G.; Anand, G. S.; Borchers, C.; Brier, S.; Bou-Assaf, G. M.; Engen, J. R.; Englander, S. W.; Faber, J.; Garlish, R.; Griffin, P. R.; Gross, M. L.; Guttman, M.; Hamuro, Y.; Heck, A. J. R.; Houde, D.; lacob, R. E.; Jorgensen, T. J. D.; Kaltashov, I. A.; Klinman, J. P.; Konermann, L.; Man, P.; Mayne, L.; Pascal, B. D.; Reichmann, D.; Skehel, M.; Snijder, J.; Strutzenberg, T. S.; Underbakke, E. S.; Wagner, C.; Wales, T. E.; Walters, B. T.; Weis, D. D.; Wilson, D. J.; Wintrode, P. L.; Zhang, Z.; Zheng, J.; Schriemer, D. C.; Rand, K. D., Recommendations for performing, interpreting and reporting hydrogen deuterium exchange mass spectrometry (HDX-MS) experiments. Nat Methods 2019, 16 (7), 595-602.

3. Ross, C. A.; Poirier, M. A., Protein aggregation and neurodegenerative disease. Nat Med 2004, 10 Suppl, S10-7.

4. Orengo, C. A.; Todd, A. E.; Thornton, J. M., From protein structure to function. Current Opinion in Structural Biology 1999, 9 (3), 374-382.

5. Shenoy, S. R.; Jayaram, B., Proteins: sequence to structure and function--current status. Curr Protein Pept Sci 2010, 11 (7), 498-514.

6. Burke, K. A.; Kauffman, K. J.; Umbaugh, C. S.; Frey, S. L.; Legleiter, J., The interaction of polyglutamine peptides with lipid membranes is regulated by flanking sequences associated with huntingtin. J Biol Chem 2013, 288 (21), 14993-5005. 
7. Wand, A. J.; Englander, S. W., Two-dimensional 1H NMR studies of cytochrome c: assignment of the N-terminal helix. Biochemistry 1986, 25 (5), 1100-6.

8. Jeng, M. F.; Englander, S. W.; Elove, G. A.; Wand, A. J.; Roder, H., Structural description of aciddenatured cytochrome $c$ by hydrogen exchange and 2D NMR. Biochemistry 1990, 29 (46), 10433-7.

9. Mainz, A.; Religa, T. L.; Sprangers, R.; Linser, R.; Kay, L. E.; Reif, B., NMR spectroscopy of soluble protein complexes at one mega-dalton and beyond. Angew Chem Int Ed Eng/ 2013, 52 (33), 8746-51.

10. Maciejko, J.; Mehler, M.; Kaur, J.; Lieblein, T.; Morgner, N.; Ouari, O.; Tordo, P.; Becker-Baldus, J.; Glaubitz, C., Visualizing Specific Cross-Protomer Interactions in the Homo-Oligomeric Membrane Protein Proteorhodopsin by Dynamic-Nuclear-Polarization-Enhanced Solid-State NMR. I Am Chem Soc 2015, 137 (28), 9032-43.

11. Buck, M.; Schwalbe, H.; Dobson, C. M., Characterization of conformational preferences in a partly folded protein by heteronuclear NMR spectroscopy: assignment and secondary structure analysis of hen egg-white lysozyme in trifluoroethanol. Biochemistry 1995, 34 (40), 13219-32.

12. Omichinski, J. G.; Clore, G. M.; Sakaguchi, K.; Appella, E.; Gronenborn, A. M., Structural characterization of a 39-residue synthetic peptide containing the two zinc binding domains from the HIV-1 p7 nucleocapsid protein by CD and NMR spectroscopy. FEBS Lett 1991, 292 (1-2), 25-30.

13. House, K. L.; Dunlap, R. B.; Odom, J. D.; Wu, Z. P.; Hilvert, D., Structural characterization of selenosubtilisin by selenium-77 NMR spectroscopy. Journal of the American Chemical Society 1992, 114 (22), 8573-8579.

14. Birnbaum, E. R.; Sykes, B. D., Nuclear magnetic resonance studies of a Ca2+-binding fragment of troponin C. Biochemistry 1978, 17 (23), 4965-71.

15. McCubbin, W. D.; Kay, C. M., Physicochemical and biological studies on the metal-induced conformational change in troponin A. Implication of carboxyl groups in the binding of calcium ion. Biochemistry 1973, 12 (21), 4228-32.

16. Ohyashiki, T.; Sekine, T., Studies on calcium ion-induced conformation changes in the actintropomyosin-troponin system by fluorimetry IV. Conformational changes in the region containing fluorescence-labeled sulfhydryl group(s) of troponin. Biochimica et Biophysica Acta (BBA) - Protein Structure 1979, 576 (1), 51-60.

17. Leavis, P. C.; Lehrer, S. S., Intrinsic fluorescence studies on troponin C. Archives of Biochemistry and Biophysics 1978, 187 (1), 243-251.

18. Lehrer, S. S.; Leavis, P. C., Fluorescence and conformational changes caused by proton binding to troponin C. Biochemical and Biophysical Research Communications 1974, 58 (1), 159-165.

19. Radford, S. E.; Dobson, C. M.; Evans, P. A., The folding of hen lysozyme involves partially structured intermediates and multiple pathways. Nature 1992, 358 (6384), 302-7.

20. Mani, R. S.; McCubbin, W. D.; Kay, C. M., Circular dichroism and fluorescence studies on troponin-tropomyosin interactions. FEBS Letters 1975, 52 (1), 127-131.

21. Meyer, T. E., Isolation and characterization of soluble cytochromes, ferredoxins and other chromophoric proteins from the halophilic phototrophic bacterium Ectothiorhodospira halophila. Biochimica et Biophysica Acta (BBA) - Bioenergetics 1985, 806 (1), 175-183.

22. Moura, I.; Bruschi, M.; Le Gall, J.; Moura, J. J. G.; Xavier, A. V., Isolation and characterization of desulforedoxin, a new type of non-heme iron protein from Desulfovibrio gigas. Biochemical and Biophysical Research Communications 1977, 75 (4), 1037-1044.

23. Jenkins, A. L.; Larsen, R. A.; Williams, T. B., Characterization of amino acids using Raman spectroscopy. Spectrochim Acta A Mol Biomol Spectrosc 2005, 61 (7), 1585-94.

24. Zhang, F.; Basinski, M. B.; Beals, J. M.; Briggs, S. L.; Churgay, L. M.; Clawson, D. K.; DiMarchi, R. D.; Furman, T. C.; Hale, J. E.; Hsiung, H. M.; Schoner, B. E.; Smith, D. P.; Zhang, X. Y.; Wery, J. P.; Schevitz, R. W., Crystal structure of the obese protein leptin-E100. Nature 1997, 387 (6629), 206-9.

25. Drenth, J., Principles of Protein X-Ray Crystallography. 2002, Third Edition. 
26. Cartailler, J. P.; Luecke, H., X-ray crystallographic analysis of lipid-protein interactions in the bacteriorhodopsin purple membrane. Annu Rev Biophys Biomol Struct 2003, 32, 285-310.

27. Zhang, X.; Walker, S. B.; Chipman, P. R.; Nibert, M. L.; Baker, T. S., Reovirus polymerase lambda 3 localized by cryo-electron microscopy of virions at a resolution of 7.6 A. Nat Struct Biol 2003, 10 (12), 1011-8.

28. Fang, Q.; Shah, S.; Liang, Y.; Zhou, Z. H., 3D reconstruction and capsid protein characterization of grass carp reovirus. Sci China C Life Sci 2005, 48 (6), 593-600.

29. Samso, M.; Shen, X.; Allen, P. D., Structural characterization of the RyR1-FKBP12 interaction. J Mol Biol 2006, 356 (4), 917-27.

30. Meng, X.; Wang, G.; Viero, C.; Wang, Q.; Mi, W.; Su, X. D.; Wagenknecht, T.; Williams, A. J.; Liu, Z.; Yin, C. C., CLIC2-RyR1 interaction and structural characterization by cryo-electron microscopy. J Mol Biol 2009, 387 (2), 320-34.

31. Limpikirati, P.; Liu, T.; Vachet, R. W., Covalent labeling-mass spectrometry with non-specific reagents for studying protein structure and interactions. Methods 2018, 144, 79-93.

32. Robinson, C. V.; Sali, A.; Baumeister, W., The molecular sociology of the cell. Nature 2007, 450 (7172), 973-82.

33. Laganowsky, A.; Reading, E.; Hopper, J. T.; Robinson, C. V., Mass spectrometry of intact membrane protein complexes. Nat Protoc 2013, 8 (4), 639-51.

34. Yu, B.; Yang, D., Coexistence of multiple minor states of fatty acid binding protein and their functional relevance. Sci Rep 2016, 6, 34171.

35. Shen, Y.; Zhao, X.; Wang, G.; Chen, D. D. Y., Differential Hydrogen/Deuterium Exchange during Proteoform Separation Enables Characterization of Conformational Differences between Coexisting Protein States. Anal Chem 2019, 91 (6), 3805-3809.

36. Esposito, L.; Donnarumma, F.; Ruggiero, A.; Leone, S.; Vitagliano, L.; Picone, D., Structure, stability and aggregation propensity of a Ribonuclease A-Onconase chimera. Int J Biol Macromol 2019, $133,1125-1133$.

37. Liu, T.; Limpikirati, P.; Vachet, R. W., Synergistic Structural Information from Covalent Labeling and Hydrogen-Deuterium Exchange Mass Spectrometry for Protein-Ligand Interactions. Anal Chem 2019, 91 (23), 15248-15254.

38. Yu, X.; Wang, J.; Ulrich, J., Solvent-freeze-out (SFO) technology: A controlled crystallization process-Case study of jack bean urease. Chemical Engineering Science 2015, 135, 137-144.

39. Dickinson, E. R.; Jurneczko, E.; Pacholarz, K. J.; Clarke, D. J.; Reeves, M.; Ball, K. L.; Hupp, T.; Campopiano, D.; Nikolova, P. V.; Barran, P. E., Insights into the conformations of three structurally diverse proteins: cytochrome $\mathrm{c}, \mathrm{p} 53$, and $\mathrm{MDM} 2$, provided by variable-temperature ion mobility mass spectrometry. Anal Chem 2015, 87 (6), 3231-8.

40. Kelstrup, C. D.; Young, C.; Lavallee, R.; Nielsen, M. L.; Olsen, J. V., Optimized fast and sensitive acquisition methods for shotgun proteomics on a quadrupole orbitrap mass spectrometer. J Proteome Res 2012, 11 (6), 3487-97.

41. Robinson, C. V.; Gross, M.; Eyles, S. J.; Ewbank, J. J.; Mayhew, M.; Hartl, F. U.; Dobson, C. M.; Radford, S. E., Conformation of GroEL-bound alpha-lactalbumin probed by mass spectrometry. Nature 1994, 372 (6507), 646-51.

42. Creighton, T., Disulphide-coupled protein folding pathways. Philos Trans $R$ Soc Lond B Biol Sci 1995, 348 (1323), 5-10.

43. Miranker, A.; Robinson, C. V.; Radford, S. E.; Dobson, C. M., Investigation of protein folding by mass spectrometry. FASEB J 1996, 10 (1), 93-101.

44. Fenn, J. B.; Mann, M.; Meng, C. K.; Wong, S. F.; Whitehouse, C. M., Electrospray ionization for mass spectrometry of large biomolecules. Science 1989, 246 (4926), 64-71. 
45. Von Helden, G.; Wyttenbach, A.; Bowers, M. T., Inclusion of a MALDI ion source in the ion chromatography technique: conformational information on polymer and biomolecular ions. Int. J. Mass Spectrom. Ion Processes 1995, 146, 349-364.

46. Clemmer, D. E.; Hudgins, R. R.; Jarrold, M. F., Naked Protein Conformations - Cytochrome-C in the Gas-Phase. J Am Chem Soc 1995, 117 (40), 10141-10142.

47. Mesleh, M. F.; Hunter, J. M.; Shvartsburg, A. A.; Schatz, G. C.; Jarrold, M. F., Structural Information from Ion Mobility Measurements: Effects of the Long-Range Potential. The Journal of Physical Chemistry 1996, 100 (40), 16082-16086.

48. Shelimov, K. B.; Clemmer, D. E.; Hudgins, R. R.; Jarrold, M. F., Protein Structure in Vacuo: Gas-Phase Conformations of BPTI and Cytochrome c. Am. Chem. Soc. 1997, 119 (9), 2240-2248.

49. Dugourd, P.; Hudgins, R. R.; Clemmer, D. E.; Jarrold, M. F., High-resolution ion mobility measurements. Rev. Sci. Instrum. 1997, 68, 1122-1129.

50. Johnson, A. R.; Dilger, J. M.; Glover, M. S.; Clemmer, D. E.; Carlson, E. E., Negatively-charged helices in the gas phase. Chem Commun (Camb) 2014, 50 (64), 8849-51.

51. Gabelica, V.; Shvartsburg, A. A.; Afonso, C.; Barran, P.; Benesch, J. L. P.; Bleiholder, C.; Bowers, M. T.; Bilbao, A.; Bush, M. F.; Campbell, J. L.; Campuzano, I. D. G.; Causon, T.; Clowers, B. H.; Creaser, C. S.; De Pauw, E.; Far, J.; Fernandez-Lima, F.; Fjeldsted, J. C.; Giles, K.; Groessl, M.; Hogan, C. J., Jr.; Hann, S.; Kim, H. I.; Kurulugama, R. T.; May, J. C.; McLean, J. A.; Pagel, K.; Richardson, K.; Ridgeway, M. E.; Rosu, F.; Sobott, F.; Thalassinos, K.; Valentine, S. J.; Wyttenbach, T., Recommendations for reporting ion mobility Mass Spectrometry measurements. Mass Spectrom Rev 2019, 38 (3), 291-320.

52. Valentine, S. J.; Counterman, A. E.; Clemmer, D. E., Conformer-dependent proton-transfer reactions of ubiquitin ions. J Am Soc Mass Spectr 1997, 8 (9), 954-961.

53. Khristenko, N.; Amato, J.; Livet, S.; Pagano, B.; Randazzo, A.; Gabelica, V., Native Ion Mobility Mass Spectrometry: When Gas-Phase Ion Structures Depend on the Electrospray Charging Process. J Am Soc Mass Spectrom 2019, 30 (6), 1069-1081.

54. Young, L. M.; Cao, P.; Raleigh, D. P.; Ashcroft, A. E.; Radford, S. E., Ion mobility spectrometrymass spectrometry defines the oligomeric intermediates in amylin amyloid formation and the mode of action of inhibitors. J Am Chem Soc 2014, 136 (2), 660-70.

55. Zucker, S. M.; Lee, S.; Webber, N.; Valentine, S. J.; Reilly, J. P.; Clemmer, D. E., An Ion Mobility/Ion Trap/Photodissociation Instrument for Characterization of Ion Structure. Journal of the American Society for Mass Spectrometry 2011, 22 (9), 1477-1485.

56. Shi, H.; Clemmer, D. E., Evidence for two new solution states of ubiquitin by IMS-MS analysis. J Phys Chem B 2014, 118 (13), 3498-506.

57. Light-Wahl, K. J.; Schwartz, B. L.; Smith, R. D., Observation of the Noncovalent Quaternary Associations of Proteins by Electrospray Ionization Mass Spectrometry. J Am Chem Soc 1994, 116 (12), 5271-5278.

58. van Berkel, W. J.; van den Heuvel, R. H.; Versluis, C.; Heck, A. J., Detection of intact megaDalton protein assemblies of vanillyl-alcohol oxidase by mass spectrometry. Protein Sci 2000, 9 (3), 435-9.

59. Wyttenbach, T.; Bowers, M. T., Structural Stability from Solution to the Gas Phase: Native Solution Structure of Ubiquitin Survives Analysis in a Solvent-Free Ion Mobility-Mass Spectrometry Environment. Journal of Physical Chemistry B 2011, 115 (42), 12266-12275.

60. Konermann, L.; Ahadi, E.; Rodriguez, A. D.; Vahidi, S., Unraveling the mechanism of electrospray ionization. Anal Chem 2013, 85 (1), 2-9.

61. Kim, D.; Wagner, N.; Wooding, K.; Clemmer, D. E.; Russell, D. H., lons from Solution to the Gas Phase: A Molecular Dynamics Simulation of the Structural Evolution of Substance P during Desolvation of Charged Nanodroplets Generated by Electrospray lonization. J Am Chem Soc 2017, 139 (8), 29812988. 
62. Rand, K. D.; Pringle, S. D.; Murphy, J. P., 3rd; Fadgen, K. E.; Brown, J.; Engen, J. R., Gas-phase hydrogen/deuterium exchange in a traveling wave ion guide for the examination of protein conformations. Anal Chem 2009, 81 (24), 10019-28.

63. Rand, K. D.; Pringle, S. D.; Morris, M.; Brown, J. M., Site-Specific Analysis of Gas-Phase Hydrogen/Deuterium Exchange of Peptides and Proteins by Electron Transfer Dissociation. Anal. Chem. 2012, 84 (4), 1931-1940.

64. Mazzitelli, C. L.; Wang, J.; Smith, S. I.; Brodbelt, J. S., Gas-phase stability of G-quadruplex DNA determined by electrospray ionization tandem mass spectrometry and molecular dynamics simulations. J Am Soc Mass Spectrom 2007, 18 (10), 1760-73.

65. Segev, E.; Wyttenbach, T.; Bowers, M. T.; Gerber, R. B., Conformational evolution of ubiquitin ions in electrospray mass spectrometry: molecular dynamics simulations at gradually increasing temperatures. Phys Chem Chem Phys 2008, 10 (21), 3077-82.

66. Saikusa, K.; Fuchigami, S.; Takahashi, K.; Asano, Y.; Nagadoi, A.; Tachiwana, H.; Kurumizaka, H.; Ikeguchi, M.; Nishimura, Y.; Akashi, S., Gas-phase structure of the histone multimers characterized by ion mobility mass spectrometry and molecular dynamics simulation. Anal Chem 2013, 85 (8), 4165-71.

67. Merkley, E. D.; Rysavy, S.; Kahraman, A.; Hafen, R. P.; Daggett, V.; Adkins, J. N., Distance restraints from crosslinking mass spectrometry: mining a molecular dynamics simulation database to evaluate lysine-lysine distances. Protein Sci 2014, 23 (6), 747-59.

68. Inutan, E. D.; Wang, B.; Trimpin, S., Commercial intermediate pressure MALDI ion mobility spectrometry mass spectrometer capable of producing highly charged laserspray ionization ions. Anal Chem 2011, 83 (3), 678-84.

69. Gabelica, V.; Marklund, E., Fundamentals of ion mobility spectrometry. Curr Opin Chem Biol 2018, 42, 51-59.

70. Donohoe, G. C.; Maleki, H.; Arndt, J. R.; Khakinejad, M.; Yi, J. H.; McBride, C.; Nurkiewicz, T. R.; Valentine, S. J., A New Ion Mobility-Linear Ion Trap Instrument for Complex Mixture Analysis. Anal. Chem. 2014, 86 (16), 8121-8128.

71. Donohoe, G. C.; Khakinejad, M.; Valentine, S. J., lon mobility spectrometry-hydrogen deuterium exchange mass spectrometry of anions: part 1. Peptides to proteins. J Am Soc Mass Spectrom 2015, 26 (4), 564-76.

72. Graves, D. B., Transport properties of ions in gases by Edward A. Mason and Earl W. McDaniel, John Wiley and Sons, New York, 1988, 560+ xvi pp. AIChE Journal 1989, 35 (4), 701-701.

73. demmers, J. A.; Haverkamp, J.; Heck, A. J.; Koeppe, R. E., 2nd; Killian, J. A., Electrospray ionization mass spectrometry as a tool to analyze hydrogen deuterium exchange kinetics of transmembrane peptides in lipid bilayers. PNAS 2000, 97 (7), 3189-3194.

74. Freitas, M. A.; Hendrickson, C. L.; Emmett, M. R.; Marshall, A. G., Gas-phase bovine ubiquitin cation conformations resolved by gas-phase hydrogen/deuterium exchange rate and extent. International Journal of Mass Spectrometry 1999, 185, 565-575.

75. Pan, J. X.; Heath, B. L.; Jockusch, R. A.; Konermann, L., Structural Interrogation of Electrosprayed Peptide Ions by Gas-Phase H/D Exchange and Electron Capture Dissociation Mass Spectrometry. Anal. Chem. 2012, 84 (1), 373-378.

76. Straus, R. N.; Jockusch, R. A., Probing the Gaseous Structure of a beta-Hairpin Peptide with H/D Exchange and Electron Capture Dissociation. J Am Soc Mass Spectrom 2017, 28 (2), 358-369.

77. Vadas, O.; Jenkins, M. L.; Dornan, G. L.; Burke, J. E., Using Hydrogen-Deuterium Exchange Mass Spectrometry to Examine Protein-Membrane Interactions. Methods Enzymol 2017, 583, 143-172.

78. Wyttenbach, T.; Bowers, M. T., Gas phase conformations of biological molecules: The hydrogen/deuterium exchange mechanism. Journal of the American Society for Mass Spectrometry 1999, 10 (1), 9-14. 
79. Zhang, H.; Cui, W.; Gross, M. L., Mass spectrometry for the biophysical characterization of therapeutic monoclonal antibodies. FEBS Lett 2014, 588 (2), 308-17.

80. Brown, K. A.; Wilson, D. J., Bottom-up hydrogen deuterium exchange mass spectrometry: data analysis and interpretation. Analyst 2017, 142 (16), 2874-2886.

81. Marcsisin, S. R.; Engen, J. R., Hydrogen exchange mass spectrometry: what is it and what can it tell us? Anal Bioanal Chem 2010, 397 (3), 967-72.

82. Prince, J. T.; Marcotte, E. M., Chromatographic alignment of ESI-LC-MS proteomics data sets by ordered bijective interpolated warping. Anal Chem 2006, 78 (17), 6140-52.

83. Qiao, J.; Wang, J.; Chen, L.; Tian, X.; Huang, S.; Ren, X.; Zhang, W., Quantitative iTRAQ LC-MS/MS proteomics reveals metabolic responses to biofuel ethanol in cyanobacterial Synechocystis sp. PCC 6803. J Proteome Res 2012, 11 (11), 5286-300.

84. Ranganathan, N.; Li, C.; Suder, T.; Karanji, A. K.; Li, X.; He, Z.; Valentine, S. J.; Li, P., Capillary Vibrating Sharp-Edge Spray Ionization (cVSSI) for Voltage-Free Liquid Chromatography-Mass Spectrometry. J Am Soc Mass Spectrom 2019, 30 (5), 824-831.

85. Rappsilber, J.; Ishihama, Y.; Mann, M., Stop and go extraction tips for matrix-assisted laser desorption/ionization, nanoelectrospray, and LC/MS sample pretreatment in proteomics. Anal Chem 2003, 75 (3), 663-70.

86. Demirev, P. A.; Feldman, A. B.; Kowalski, P.; Lin, J. S., Top-down proteomics for rapid identification of intact microorganisms. Anal Chem 2005, 77 (22), 7455-61.

87. Toby, T. K.; Fornelli, L.; Kelleher, N. L., Progress in Top-Down Proteomics and the Analysis of Proteoforms. Annu Rev Anal Chem (Palo Alto Calif) 2016, 9 (1), 499-519.

88. Tran, J. C.; Zamdborg, L.; Ahlf, D. R.; Lee, J. E.; Catherman, A. D.; Durbin, K. R.; Tipton, J. D.; Vellaichamy, A.; Kellie, J. F.; Li, M.; Wu, C.; Sweet, S. M.; Early, B. P.; Siuti, N.; LeDuc, R. D.; Compton, P. D.; Thomas, P. M.; Kelleher, N. L., Mapping intact protein isoforms in discovery mode using top-down proteomics. Nature 2011, 480 (7376), 254-8.

89. Karanji, A. K.; Khakinejad, M.; Kondalaji, S. G.; Majuta, S. N.; Attanayake, K.; Valentine, S. J., Comparison of Peptide Ion Conformers Arising from Non-Helical and Helical Peptides Using lon Mobility Spectrometry and Gas-Phase Hydrogen/Deuterium Exchange. J Am Soc Mass Spectrom 2018, 29 (12), 2402-2412.

90. Khakinejad, M.; Ghassabi Kondalaji, S.; Tafreshian, A.; Valentine, S. J., Comprehensive Gas-Phase Peptide Ion Structure Studies Using Ion Mobility Techniques: Part 2. Gas-Phase Hydrogen/Deuterium Exchange for lon Population Estimation. J Am Soc Mass Spectrom 2017, 28 (5), 960-970.

91. Khakinejad, M.; Kondalaji, S. G.; Tafreshian, A.; Valentine, S. J., Gas-Phase Hydrogen-Deuterium Exchange Labeling of Select Peptide Ion Conformer Types: a Per-Residue Kinetics Analysis. J Am Soc Mass Spectrom 2015.

92. Lindh, E. L.; Salmén, L., Surface accessibility of cellulose fibrils studied by hydrogen-deuterium exchange with water. Cellulose 2016, 24 (1), 21-33.

93. Winters, M. S.; Spellman, D. S.; Lambris, J. D., Solvent accessibility of native and hydrolyzed human complement protein 3 analyzed by hydrogen/deuterium exchange and mass spectrometry. $J$ Immunol 2005, 174 (6), 3469-74.

94. Mistarz, U. H.; Bellina, B.; Jensen, P. F.; Brown, J. M.; Barran, P. E.; Rand, K. D., UV Photodissociation Mass Spectrometry Accurately Localize Sites of Backbone Deuteration in Peptides. Anal Chem 2018, 90 (2), 1077-1080.

95. Donohoe, G. C.; Arndt, J. R.; Valentine, S. J., Online deuterium hydrogen exchange and protein digestion coupled with ion mobility spectrometry and tandem mass spectrometry. Anal Chem 2015, 87 (10), 5247-54. 
96. Majuta, S. N.; Maleki, H.; Kiani Karanji, A.; Attanyake, K.; Loch, E.; Valentine, S. J., Magnifying ion mobility spectrometry-mass spectrometry measurements for biomolecular structure studies. Curr Opin Chem Biol 2018, 42, 101-110.

97. Zhang, Y.; Fonslow, B. R.; Shan, B.; Baek, M. C.; Yates, J. R., 3rd, Protein analysis by shotgun/bottom-up proteomics. Chem Rev 2013, 113 (4), 2343-94.

98. Gillet, L. C.; Leitner, A.; Aebersold, R., Mass Spectrometry Applied to Bottom-Up Proteomics: Entering the High-Throughput Era for Hypothesis Testing. Annu Rev Anal Chem (Palo Alto Calif) 2016, 9 (1), 449-72.

99. Savaryn, J. P.; Catherman, A. D.; Thomas, P. M.; Abecassis, M. M.; Kelleher, N. L., The emergence of top-down proteomics in clinical research. Genome Med 2013, 5 (6), 53.

100. Khakinejad, M.; Kondalaji, S. G.; Maleki, H.; Arndt, J. R.; Donohoe, G. C.; Valentine, S. J., Combining ion mobility spectrometry with hydrogen-deuterium exchange and top-down MS for peptide ion structure analysis. J Am Soc Mass Spectrom 2014, 25 (12), 2103-15.

101. Mistarz, U. H.; Brown, J. M.; Haselmann, K. F.; Rand, K. D., Simple Setup for Gas-Phase H/D Exchange Mass Spectrometry Coupled to Electron Transfer Dissociation and Ion Mobility for Analysis of Polypeptide Structure on a Liquid Chromatographic Time Scale. Anal. Chem. 2014, 86 (23), 11868-11876. 102. Rand, K. D.; Adams, C. M.; Zubarev, R. A.; Jorgensen, T. J., Electron capture dissociation proceeds with a low degree of intramolecular migration of peptide amide hydrogens. J Am Chem Soc 2008, 130 (4), 1341-9.

103. Zehl, M.; Rand, K. D.; Jensen, O. N.; Jorgensen, T. J., Electron transfer dissociation facilitates the measurement of deuterium incorporation into selectively labeled peptides with single residue resolution. J Am Chem Soc 2008, 130 (51), 17453-9.

104. van der Ploeg, P.; Berendsen, H. J. C., Molecular dynamics simulation of a bilayer membrane. The Journal of Chemical Physics 1982, 76 (6), 3271-3276.

105. Haider, A.; Wei, Y. C.; Lim, K.; Barbosa, A. D.; Liu, C. H.; Weber, U.; Mlodzik, M.; Oras, K.; Collier, S.; Hussain, M. M.; Dong, L.; Patel, S.; Alvarez-Guaita, A.; Saudek, V.; Jenkins, B. J.; Koulman, A.; Dymond, M. K.; Hardie, R. C.; Siniossoglou, S.; Savage, D. B., PCYT1A Regulates Phosphatidylcholine Homeostasis from the Inner Nuclear Membrane in Response to Membrane Stored Curvature Elastic Stress. Dev Cell 2018, 45 (4), 481-495 e8.

106. Karanji, A. K.; Beasley, M.; Sharif, D.; Ranjbaran, A.; Legleiter, J.; Valentine, S. J., Investigating the interactions of the first 17 amino acid residues of Huntingtin with lipid vesicles using mass spectrometry and molecular dynamics. J Mass Spectrom 2019.

107. Chen, M.; Wolynes, P. G., Aggregation landscapes of Huntingtin exon 1 protein fragments and the critical repeat length for the onset of Huntington's disease. Proc Natl Acad Sci U S A 2017, 114 (17), 4406-4411.

108. Arndt, J. R.; Chaibva, M.; Beasley, M.; Kiani Karanji, A.; Ghassabi Kondalaji, S.; Khakinejad, M.; Sarver, O.; Legleiter, J.; Valentine, S. J., Nucleation Inhibition of Huntingtin Protein (htt) by Polyproline PPII Helices: A Potential Interaction with the N-Terminal alpha-Helical Region of Htt. Biochemistry 2019.

109. Arndt, J. R.; Kondalaji, S. G.; Maurer, M. M.; Parker, A.; Legleiter, J.; Valentine, S. J., Huntingtin NTerminal Monomeric and Multimeric Structures Destabilized by Covalent Modification of Heteroatomic Residues. Biochemistry 2015, 54 (28), 4285-96.

110. Jayaraman, M.; Mishra, R.; Kodali, R.; Thakur, A. K.; Koharudin, L. M.; Gronenborn, A. M.; Wetzel, R., Kinetically competing huntingtin aggregation pathways control amyloid polymorphism and properties. Biochemistry 2012, 51 (13), 2706-16.

111. Kegel, K. B.; Sapp, E.; Yoder, J.; Cuiffo, B.; Sobin, L.; Kim, Y. J.; Qin, Z. H.; Hayden, M. R.; Aronin, N.; Scott, D. L.; Isenberg, G.; Goldmann, W. H.; DiFiglia, M., Huntingtin associates with acidic phospholipids at the plasma membrane. J Biol Chem 2005, 280 (43), 36464-73. 
112. Levy, G. R.; Shen, K.; Gavrilov, Y.; Smith, P. E. S.; Levy, Y.; Chan, R.; Frydman, J.; Frydman, L., Huntingtin's N-Terminus Rearrangements in the Presence of Membranes: A Joint Spectroscopic and Computational Perspective. ACS Chem Neurosci 2018.

113. Chaibva, M.; Burke, K. A.; Legleiter, J., Curvature enhances binding and aggregation of huntingtin at lipid membranes. Biochemistry 2014, 53 (14), 2355-65.

114. Suopanki, J.; Gotz, C.; Lutsch, G.; Schiller, J.; Harjes, P.; Herrmann, A.; Wanker, E. E., Interaction of huntingtin fragments with brain membranes--clues to early dysfunction in Huntington's disease. $J$ Neurochem 2006, 96 (3), 870-84.

115. Adegbuyiro, A.; Sedighi, F.; Pilkington, A. W. t.; Groover, S.; Legleiter, J., Proteins Containing Expanded Polyglutamine Tracts and Neurodegenerative Disease. Biochemistry 2017, 56 (9), 1199-1217.

116. Cote, S.; Binette, V.; Salnikov, E. S.; Bechinger, B.; Mousseau, N., Probing the Huntingtin 1-17 membrane anchor on a phospholipid bilayer by using all-atom simulations. Biophys $J$ 2015, 108 (5), 1187-98.

117. Kegel, K. B.; Sapp, E.; Alexander, J.; Valencia, A.; Reeves, P.; Li, X.; Masso, N.; Sobin, L.; Aronin, N.; Difiglia, M., Polyglutamine expansion in huntingtin alters its interaction with phospholipids. J Neurochem 2009, 110 (5), 1585-97.

118. Atwal, R. S.; Xia, J.; Pinchev, D.; Taylor, J.; Epand, R. M.; Truant, R., Huntingtin has a membrane association signal that can modulate huntingtin aggregation, nuclear entry and toxicity. Hum Mol Genet 2007, 16 (21), 2600-15.

119. Faramarzi, S.; Feng, J.; Mertz, B., Allosteric Effects of the Proton Donor on the Microbial Proton Pump Proteorhodopsin. Biophys J 2018, 115 (7), 1240-1250.

120. Ran, T.; Ozorowski, G.; Gao, Y.; Sineshchekov, O. A.; Wang, W.; Spudich, J. L.; Luecke, H., Crossprotomer interaction with the photoactive site in oligomeric proteorhodopsin complexes. Acta Crystallogr D Biol Crystallogr 2013, 69 (Pt 10), 1965-80. 


\section{Comparison of Peptide lon Conformers Arising from Non- Helical and Helical Peptides Using Ion Mobility Spectrometry and Gas-Phase Hydrogen/Deuterium Exchange}

Reprinted with permission from Journal of American Society for Mass Spectrometry: Comparison of Peptide Ion Conformers Arising from Non-Helical and Helical Peptides Using Ion Mobility Spectrometry and Gas-Phase Hydrogen/Deuterium Exchange. Ahmad Kiani Karanji, Mahdiar Khakinejad, Samaneh Ghassabi Kondalaji, Sandra N. Majuta, Kushani Attanayake, Stephen J. Valentine. J. Am. Soc. Mass Spectrom. 2018, 29, 12, 2402-2412.

\section{Introduction}

Since the discovery of the structure of the a-helix in polypeptides, ${ }^{1}$ significant research has been devoted to determining factors that affect the formation of elements of secondary structure and in particular those effecting helix formation ${ }^{2}$. Initial efforts, boosted by the expanding available protein structure data, focused on the relationship between the presence of a-helices and the frequency of occurrence of specific amino acid residues ${ }^{3-7}$. Shortly later, Chou and Fasman proposed a method for predicting this secondary structure element using amino acid sequence information ${ }^{8}$. Over the next several decades, interest in helix structure prediction progressed to the point where a relatively high degree of accuracy was obtained ${ }^{9-10}$. This progress coincided with efforts to refine helix propensities of the individual amino acid residues $^{2,11-12}$ and the concept has even been extended to structure destabilization imparted by D-amino acid residues ${ }^{13-14}$.

The rapid increase in the number of experimentally-determined protein structures over the last three decades ${ }^{15-16}$ has significantly enabled protein structure prediction capabilities. Perhaps, the simplest conception is to use sequence homology based on comparisons to known structures to infer structural elements for proteins of interest ${ }^{17-19}$. Over the years other computational techniques have advanced significantly and protein structure prediction efforts can currently be grouped into those using ab initio methods (or ab initio guided approaches) and 
template-based methods (fold recognition and threading, comparative modelling) ${ }^{20}$. Currently, protein structure prediction remains a highly active and rapidly evolving area of research ${ }^{21-23}$.

A challenge in the characterization of polypeptide structure are species that have been designated as intrinsically disordered ${ }^{24-26}$. Although efforts have been developed for predicting unstructured regions of proteins ${ }^{27-28}$, recent developments regarding the nature of species classified as intrinsically disordered have called into question whether or not structural assessments are possible. One area of interest is the study of peptide structure. For example, the peptide bradykinin was examined by NMR many years ago and only a small portion of the molecule was assigned to a $\beta$-turn structural type ${ }^{29}$. Recent experiments that employed ion mobility spectrometry (IMS) combined with mass spectrometry (MS) have shed some light on the inability of solution-phase measurements to determine prevailing structural motifs for peptides; for bradykinin, these experiments revealed the presence of multiple, co-existing solution structures ${ }^{30-31}$. Although the exact relationship of gas-phase structures to antecedent solution states is still under investigation, such efforts suggested that gas-phase analyses could provide insight into the various structures of polypeptides that exist in solution. Interestingly, extending the approach has suggested the presence of multiple protein structures for species for which well-defined native conformations predominate ${ }^{32}$.

Results from the early IMS-MS experiments lead to questions regarding the degree to which elements of secondary structure are preserved in the gas-phase for such dynamic systems and whether or not solution structures can be derived from high-quality gas-phase structures. To study this, recent experiments have examined the conformations of $[\mathrm{M}+3 \mathrm{H}]^{3+}$ ions of the model peptide Acetyl-PAAAAKAAAAKAAAAKAAAAK (hereafter referred to as the Acontaining peptide) using a combination of IMS-MS and gas-phase hydrogen/deuterium exchange (HDX) measurements and molecular modeling techniques ${ }^{33-35}$. This peptide was selected because of its relatively large propensity to form a helix in solution ${ }^{2,36-37}$. In general, 
studies suggested that the presence of multiple conformer types of varying degree of helicity can contribute to the $[\mathrm{M}+3 \mathrm{H}]^{3+}$ ion conformers that were studied ${ }^{33}$. In many respects, this work was similar in nature to early efforts aimed at designing gas-phase peptide ion structure ${ }^{38}$ as well as studies that have characterized peptide structural motifs with gas-phase HDX ${ }^{39-40}$.

The present work examines the $[\mathrm{M}+3 \mathrm{H}]^{3+}$ ions of the model peptide AcetylPSSSSKSSSSKSSSSKSSSSK (hereafter referred to as the S-containing peptide). This peptide has been designed to exhibit less helical propensity in solution ${ }^{2}$. Indeed, because the hydroxyl oxygen of serine $(S)$ can hydrogen bond with the carbonyl oxygen of the $3^{\text {rd }}$ residue, $S$ is known to destabilize $\alpha$-helix structure ${ }^{41}$. Additionally, regions of high $\mathrm{S}$ density are associated with helix-breaking structure ${ }^{42}$. The model peptide was also designed because of the potential to experience a different process of gas-phase conformation establishment when compared with the previously studied A-containing peptide. Previous work has suggested that the non-polar A residues may strongly associate with the a polar vacuum environment during the final stages of $\mathrm{ESI}{ }^{35,43}$; it may be expected that the $\mathrm{S}$ residues will more strongly favor solvent interaction during the final stages of ESI. These differences offer the opportunity to compare the ion structure types formed by very different solution conformers and possibly by different processes of gas-phase conformer establishment.

Overall, ion collision cross section values are nearly the same compared with the Acontaining peptide. However, because the S-containing peptide has a larger molecular weight, one may conclude the overall structure is more compact. Additionally, there are significant differences in the relative accessibility of exchange sites of terminal and middle amino acid residues with the latter being more accessible to the S-containing peptide. These characteristics are discussed below using structures obtained from simulated annealing to help provide insight into relative accessibility. By providing information about gas-phase ion conformer establishment from different solution states, these efforts may help to enhance protein structure 
prediction tools in the future. Finally, this work adds to the growing repertoire of studies that employ IMS-MS, gas-phase HDX, and/or MD simulation techniques for peptide/protein ion structure characterization ${ }^{44-53}$.

\section{Experimental}

Sample Preparation. The model peptide acetyl-PSSSSKSSSSKSSSSKSSSSK $(97.3 \%$ purity) was purchased from GenScript (Piscataway, NJ, USA) and used without further purification. To prepare the $1 \mathrm{mg} / \mathrm{mL}$ stock solution, the peptide was dissolved in the appropriate amount of Milli-Q water. The stock solution was then diluted 10 fold with a $100 \mathrm{mM}$ solution of ammonium acetate in water to prepare the ESI solutions. This solution was infused $(0.5 \mu \mathrm{L} / \mathrm{min})$ through a pulled-tipped capillary which was biased $\sim+2200 \mathrm{~V}$ relative to the entrance of the hybrid IMS-MS instrument.

IMS-HDX-MS Measurements. For the ion mobility measurements conducted here, a dual-gate drift tube similar to others described in the literature was used ${ }^{54-55}$. The details of the drift time $\left(t_{D}\right)$ and MS measurements have been described in detail previously ${ }^{56-57}$. Briefly, for this study, ions generated by ESI were periodically $(50 \mathrm{~Hz})$ pulsed into a 1-m-long drift tube that was filled with $\sim 2.5$ Torr of $300 \mathrm{~K}$ helium buffer gas. For the dual ion gate approach, the second gate was set to transmit at specific delay times in order to record mobility-resolved mass spectra. This delay time was scanned $(200 \mu$ increments $)$ across the mobility distribution of the $[\mathrm{M}+3 \mathrm{H}]^{3+}$ ions. Mass spectra were recorded by a linear ion trap (LTQ Velos; ThermoScientific, San Jose, CA, USA) instrument. A mass-to-charge $(\mathrm{m} / \mathrm{z})$ range of 400 to 1000 was employed. For each $t_{D}$ increment mass spectra were recorded for $0.5 \mathrm{~min}$. For mobility selection, the second ion gate was set to transmit for $200 \mu \mathrm{s}$. 
The HDX measurements conducted here are modeled after foundational work in which the reactivities of different biomolecular ions were examined upon reaction with gaseous deuterating reagent ${ }^{58-65}$. For HDX measurements, $\sim 0.02$ Torr of $\mathrm{D}_{2} \mathrm{O}$ was added to the buffer gas. With the addition of $\mathrm{D}_{2} \mathrm{O}$, the $t_{D}$ distributions shifted to longer times as shown in Figure 2.1. Therefore, for the tandem MS (MS/MS) measurements, the delay times were scanned across the $t_{D}$ distributions obtained when employing $\mathrm{D}_{2} \mathrm{O}$ to determine the mobility selection time corresponding with the dominant, compact conformer type for the $[\mathrm{M}+3 \mathrm{H}]^{3+}$ ions. As it was desired to examine the HDX behavior of the dominant, most compact ion conformer type, mobility selection was initiated $200 \mu$ s prior to the peak maximum time point in order to minimize contamination from larger ions (Figure 2.1). 


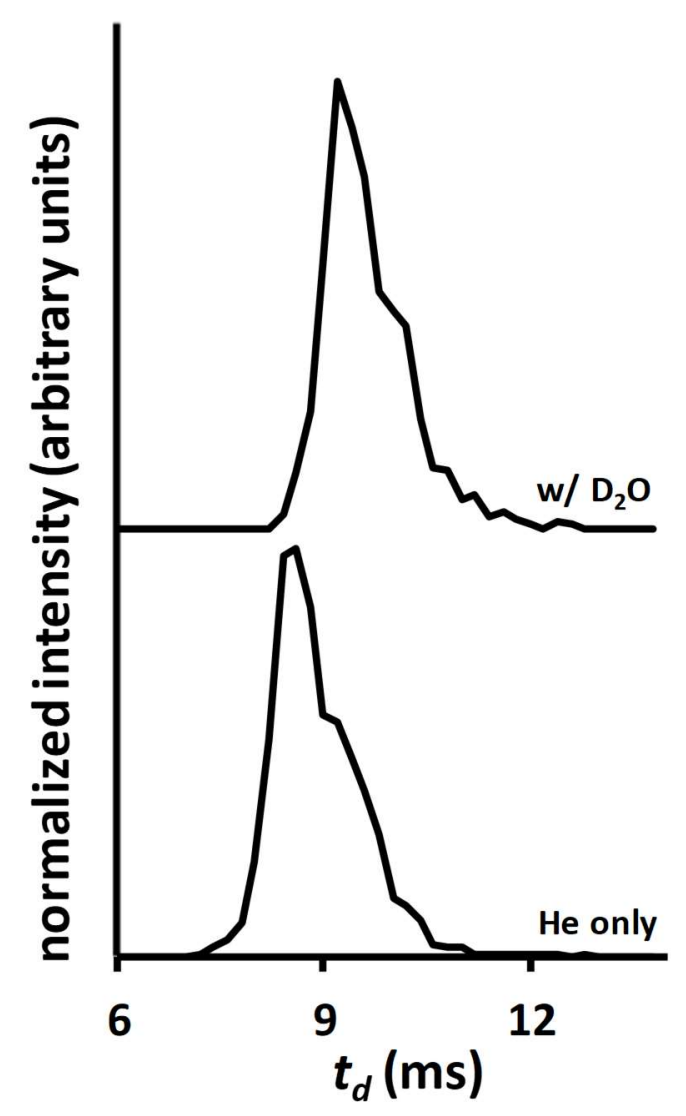

Figure 2. $1 \mathrm{t}_{D}$ distributions for the $[\mathrm{M}+3 \mathrm{H}]^{3+}$ ions of the model peptide AcetylPSSSSKSSSSKSSSSKSSSSK.

The bottom and top traces show the distributions obtained in the absence of $D_{2} \mathrm{O}$ and after the introduction of $\sim 0.02$ Torr $\mathrm{D}_{2} \mathrm{O}$. For both distributions, a delay step time of $200 \mu$ s was used and each mobility-resolved spectrum was collected for $0.5 \mathrm{~min}$. Integration of the isotopic envelope for the $[\mathrm{M}+3 \mathrm{H}]^{3+}$ ions at each delay time setting provided the intensity values for the distributions. The distributions have been normalized to show relative intensities. The red bar in the top trace shows the typical mobility selection time and width. 
Peptide Ion Dissociation by ETD and Per-Residue Deuterium Uptake Calculations. The MS/MS analyses described here have also been influenced by foundational work demonstrating the utility of MS/MS to determine deuterium uptake from gas- and solution-phase experiments ${ }^{66-68}$. Briefly, mobility-selected $[\mathrm{M}+3 \mathrm{H}]^{3+}$ ions were subjected to electron transfer dissociation (ETD) ${ }^{69}$ to determine the per-residue deuterium incorporation as described previously ${ }^{56,70}$. To perform ETD measurements, mobility-selected ions were isolated by $\mathrm{m} / \mathrm{z}$ and the ion injection time was maintained at $200 \mathrm{~ms}$ (5 microscans). Because ETD did not generate a number of lower $\mathrm{m} / \mathrm{z}$ ions, the deuterium uptake value for each residue was obtained using both $z$ - and $c$-type ions. The per-residue deuterium uptake values for the fragment ions were obtained by first subtracting the average mass of the fragment ions obtained in the absence of $D_{2} \mathrm{O}$ from the average mass of those generated upon using $\mathrm{D}_{2} \mathrm{O}$ reagent gas. For the triplicate measurements, the amount of deuterium incorporated in each residue was calculated by subtracting the deuterium uptake values of the appropriate adjacent fragment ions. Here it is noted that mass spectrometer conditions including all focusing optics were maintained at conditions that have been shown to not induce deuterium scrambling ${ }^{71}$.

Molecular Dynamics (MD) Simulations. Details regarding the in-vacuo MD simulations can also be found in the prior, related work ${ }^{72}$. Briefly, the initial structures of the $[\mathrm{M}+3 \mathrm{H}]^{3+}$ ions for simulated annealing were generated using the Visual Molecular Dynamics (VMD). The CHARMM36 force field was employed for structural parameterization. All of the parameters were defined in this force field. The CNEU patch in the top_all36_prot topology file were used to protonate the $\mathrm{C}$-terminal $\mathrm{K}$ residue resulting in $\mathrm{a}+1$ formal charge. The LSN patch was used to protonate the more $\mathrm{N}$-terminal $\mathrm{K}$ residues. Acetyl groups were attached to the $\mathrm{N}$-terminus portion of the $\mathrm{P}$ residue by using the ACP patch. 5000 steps of geometry and energy minimization were carried out using the Nanoscale Molecular Dynamics (NAMD) software suite for the $[M+3 H]^{3+}$ peptide ions with charge arrangements of $K(6)-K(11)-K(16), K(6)-K(11)-K(21)$, 
$\mathrm{K}(6)-\mathrm{K}(16)-\mathrm{K}(21)$ and $\mathrm{K}(11)-\mathrm{K}(16)-\mathrm{K}(21)$. Then, the structures were subjected to cyclic simulated annealing (SA) for conformational space sampling. For SA runs (timestep of $1 \mathrm{fs}$ ), the Berendsen temperature coupling algorithm was used. For the SA algorithm, the temperature of the in-vacuo system was suddenly increased to $1010 \mathrm{~K}$ over 10 steps. The heated structure was maintained at that temperature for $40 \mathrm{ps}$ and then cooled to a lower temperature $(10 \mathrm{~K})$ over two different timescales (40 and 1200 ps). The annealed structures were subsequently energy minimized to $0 \mathrm{~K}$ to generate candidate structures. The energy minimized structure of each cycle was imported as the starting point for the next heating-cooling cycle. Each minimized structure was gradually heated to $300 \mathrm{~K}$ and equilibrated. The structures then undergo $5 \mathrm{~ns}$ of MD simulations with no non-bonded cutoffs for long-range interactions while the temperature was constant. The Mobcal software suite ${ }^{73}$ was used to calculate theoretical collision crosssection values using the trajectory method ${ }^{74}$.

Hydrogen Accessibility Scoring (HAS) and HDX Kinetics Modeling. HAS scores were calculated for in-silico structures with matching collision cross sections. Two candidate structures which contained CCS values matching experimental measurements were selected for discussion. The selection was based on a first-pass examination of HAS scores for which one ion structure displayed increased proximity of charge sites to middle residues while the other displayed less access to these residues (see discussion below) as is suggested to be required by the "relay" mechanism for HDX ${ }^{75}$. Hydrogen accessibility was computed as described previously ${ }^{76}$ where each carbonyl site was scored based on its relative access to charge sites on the hypothetical peptide ions. Then the distances between exchange sites and these scored carbonyls were used to obtain relative theoretical reactivities of each exchange site which were summed for individual residues to provide a residue score. Finally, the residue scores were scaled based on relative values of surface accessibility using the solvent accessibility surface area (SASA) approach in the VMD software suite. Using these residue scores, the deuterium incorporation 
was estimated for $[\mathrm{M}+3 \mathrm{H}]^{3+}$ ions using an HDX kinetics model described previously ${ }^{56}$. Briefly, a population of ions (1000) was stepped $(1 \mu \mathrm{s})$ through a mobility separation $(\sim 10 \mathrm{~ms})$ and the exchange nature of each site was computed at each step based on the contribution of the residue to the overall rate of exchange for the ions. After stepping all ions through the mobility separation time, the HDX simulation algorithm determined the amount of deuterium incorporation for each residue; this theoretical uptake value is compared to the experimental results below.

\section{Results and Discussion}

Peptide Ion Collision Cross Sections. Summing the spectral counts for the $[\mathrm{M}+3 \mathrm{H}]^{3+}$ peptide ions at each mobility selection allows the generation of the $t_{D}$ distribution shown in Figure 2.1 (bottom trace). The distribution is dominated by a feature having a $t_{D}$ of $\sim 8.5 \mathrm{~ms}$. A shoulder, indicating the presence of larger ion conformer types is also observed. With the addition of $\sim 0.02$ Torr of $\mathrm{D}_{2} \mathrm{O}$, the entire distribution shifts to longer times. The dominant peak is observed to have a $t_{D}$ of $\sim 9.3 \mathrm{~ms}$ representing a shift of $\sim 0.8 \mathrm{~ms}$. Again, a shoulder of similar intensity is observed at longer $t_{D}$ values. The shift in the shoulder feature is also observed to be of the same magnitude. That the distribution is similar in the presence and absence of $\mathrm{D}_{2} \mathrm{O}$ suggests that the HDX process does not result in structural transformations and it is therefore possible to monitor the reactivities of select ion conformer types. This is similar to results that have been presented for protein ions ${ }^{60}$.

From the ion $t_{D}$ distribution, it is possible to determine the collision cross section of the $[\mathrm{M}+3 \mathrm{H}]^{3+}$ ions. Collision cross sections are determined using Equation $1^{77}$ :

$$
\Omega=\frac{(18 \pi)^{1 / 2}}{16} \frac{z e}{\left(k_{B} T\right)^{1 / 2}}\left[\frac{1}{m_{I}}+\frac{1}{m_{B}}\right]^{1 / 2} \frac{t_{D} E}{L} \frac{760}{P} \frac{T}{273.2} \frac{1}{N} \quad \text { Equation 2. } 1
$$


In Equation 1, ze and $k_{B}$ are the charge of the ion and Boltzmann's constant, respectively. The variables $m_{l}$ and $m_{B}$ are the mass of the ion and the mass of the buffer gas, respectively. $E, L$, $T$, and $P$ represent the electric field, the length of the drift tube, and the temperature and pressure of the buffer gas, respectively. Finally, $N$ is the neutral number density at standard temperature and pressure (STP) conditions. Using Equation 1, the ${ }^{\mathrm{He}} \Omega_{\mathrm{DT}}(\mathrm{DT}=$ linear drift tube) is determined to be $424 \AA^{2}$ and $464 \AA^{2}$ for the prominent feature and shoulder, respectively. These values are larger but similar to those determined for more compact and partially-unfolded A-containing peptide conformers $\left(417 \AA^{2} \text { and } 438 \AA^{2}\right)^{34,76}$.

A goal in examining the model peptide ions is to begin to determine the degree of similarity in solution- and gas-phase peptide structure in order to better understand ion conformer establishment. A number of studies suggest that a high degree of structure preservation is observed for relatively small proteins ${ }^{78-79}$. The fate of even smaller peptide ions is less clear. As mentioned above, comparisons of the data reported here for the S-containing peptide are made with those obtained from previous studies of the A-containing peptide because of expected differences in predominant solution conformers. Indeed, CD spectroscopy of the Acontaining peptide suggests a high-degree of helical character as indicated by the negative bands at $\sim 207$ and $223 \mathrm{~nm}$ as well as the positive band at $\sim 190 \mathrm{~nm}$ shown in Figure $\mathrm{S} 1$ in the Supplementary Information section (Appendix A). The CD spectrum for the S-containing peptide (Figure S1 in the Supplementary Information section (Appendix A)) shows a very clear difference in predominant solution structure for this peptide where the features indicating a-helix are clearly missing and a broad band at lower wavelengths is indicative of more random coil character.

Because of these differences in solution structure, it is of interest to consider the compact ion conformers for both the S- and A-containing peptides. Notably, the collision cross section of the S-containing peptide is only $\sim 1.7 \%$ larger than that of the A-containing peptide ion (see above). 
This occurs even though the molecular weight of the S-containing peptide is $\sim 14.1 \%$ larger than that of the comparative peptide. Thus, the overall gas-phase "packing" of the S-containing peptide is more extensive than that of the A-containing peptide. This increased atom density for the S-containing peptide ions offers the opportunity to determine whether or not distinctive HDX behavior can be observed that would be consistent with such ion structures. Thus for the Scontaining peptide, the residue specific deuterium content was examined in detail; the deuterium content was then compared to that of the A-containing peptide and the results are discussed below. Here it is noted that it was not possible to compare the more elongated conformer of the $[\mathrm{M}+3 \mathrm{H}]^{3+}$ ions as the signal level for the mobility-selected ions was insufficient to obtain ion fragmentation spectra.

Per-residue Deuterium Uptake. Upon introduction of $\mathrm{D}_{2} \mathrm{O}$ to the drift tube, the $\mathrm{m} / \mathrm{z}$ of the $[\mathrm{M}+3 \mathrm{H}]^{3+}$ ions shifted to higher values $(\mathrm{m} / \mathrm{z}$ 695.4) indicating the incorporation of 20.1 deuteriums (19.9 \pm 0.9 , on average). Overall there are 48 exchangeable hydrogens (including the three protonation sites). Thus, $\sim 41 \%$ of the exchangeable hydrogens are observed to undergo exchange. This is similar to the level of deuterium incorporation observed for the Acontaining peptide ${ }^{76}$. Therefore, it is useful to consider the amount of deuterium incorporation occurring at the neutral S and A residues. Notably, because the ETD approach used here does not differentiate between side-chain and backbone sites, the question of relative accessibility of different site types for the $S$ residues cannot currently be answered. However, a semiquantitative comparison of residue accessibility can still be achieved based on the per-residue deuterium content.

To determine the S-residue deuterium content for the gas-phase HDX studies, the more compact $[\mathrm{M}+3 \mathrm{H}]^{3+}$ precursor ions were mobility selected and subjected to ETD both before and after introduction of the $\mathrm{D}_{2} \mathrm{O}$ reagent gas. Figure $2.2 \mathrm{a}$ shows the fragmentation spectrum produced for the unlabeled ions. Two strong features are observed at $m / z \sim 688.7$ and 
$\mathrm{m} / \mathrm{z} 1032.5$ and correspond to the $[\mathrm{M}+3 \mathrm{H}]^{3+}$ precursor ions and the charge-reduced, doublycharged ions. Overall, a general lack of low- $\mathrm{m} / \mathrm{z}$ ions is observed in the fragmentation spectrum; dataset features for the $z_{4}$ through $z_{20}$ and the $c_{6}$ through $c_{20}$ fragment ions are observed. Observed ions used in deuterium content calculations with their associated $\mathrm{m} / \mathrm{z}$ values are provided in Table S1 in the Supplementary Information section (Appendix A). Because many lower $\mathrm{m} / \mathrm{z}$ fragment ions are not observed, the deuterium uptake values for individual amino acid residues have been determined using a combination of $c$ and $z$ ions. There are two advantages in using both $c$ and $z$ ions to obtain the uptake values. The first is that nearly complete sequence coverage can be obtained and the second is that a double check of deuterium uptake is provided especially for dataset features of lower intensity.

After undergoing gas-phase HDX, the fragment ions produced by ETD broaden due to an increased number of isotopologues and shift to higher $\mathrm{m} / \mathrm{z}$ values as shown in Figure $2.2 \mathrm{~b}$. In the case of the $z$ ions, by subtracting the $z_{i-1}$ ion from the $z_{i}$, the deuterium uptake value for the residue numbered $N-i-1$ can be determined for which $N$ is the number of total peptide residues (21 in this case). For the $c$ ions, by measuring the difference between the $c_{i-1}$ and the $c_{i}$ ions, the deuterium content of the residue number $i$ can be calculated. For example, the average $\mathrm{m} / \mathrm{z}$ for the peak corresponding to $z_{15}$ ions is 1432.68 and the same peak when $D_{2} \mathrm{O}$ is introduced is $\mathrm{m} / \mathrm{z}$ 1445.40. Thus the deuterium content is calculated to be 12.72 . Another example in Figure 2.2 is the $c_{6}$ ions. In this case the average $\mathrm{m} / \mathrm{z}$ is 633.70 and the corresponding average $\mathrm{m} / \mathrm{z}$ in the presence of $\mathrm{D}_{2} \mathrm{O}$ is 638.78 . Here the deuterium content is 5.08. Table S1 in the Supplementary Information section (Appendix A) provides the deuterium uptake values for the amino acid residues obtained from the $z$ and $c$ ion fragments. 


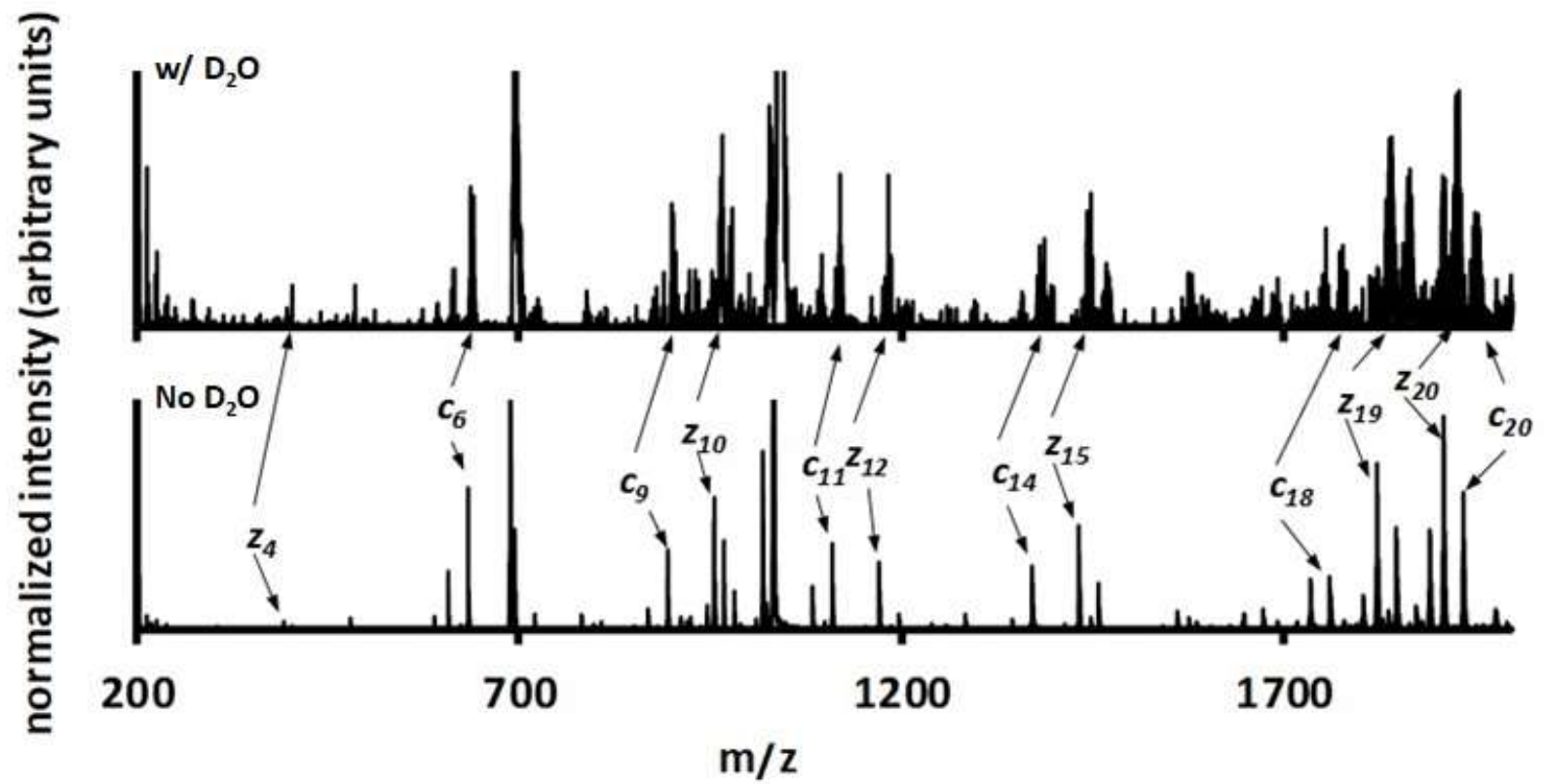

Figure 2. 2 lon fragmentation spectra obtained upon ETD of the $[\mathrm{M}+3 \mathrm{H}] 3+$ ions of the model peptide Acetyl-PSSSSKSSSSKSSSSKSSSSK.

a) Shows a typical ion fragmentation spectrum for ions that are not exposed to $D_{2} O$ in the drift tube. b) Shows a typical ion fragmentation spectrum for ions that have been exposed to $D_{2} O$ in the drift tube. Several $c$ and $z$ ions are labeled with arrows indicating their location in both spectra. 
To provide further insight into the determination of deuterium uptake, it is instructive to examine expanded regions of the mass spectra shown in Figure 2.2. Figure S2 in the Supplementary Information section (Appendix A) shows expanded regions for three fragment ions. In general, as the fragment ion mass increases, an increased shift to higher $\mathrm{m} / \mathrm{z}$ is observed and a wider isotopic distribution is encountered for ions produced after HDX (Figure S2 in the Supplementary Information section (Appendix A)). For fragment ions produced after HDX, the average $\mathrm{m} / \mathrm{z}$ value is computed by locating the dataset feature edges (typically $\leq 20 \%$ peak height) and then computing the weighted average of the isotope peaks between the edges. The average $\mathrm{m} / \mathrm{z}$ of the fragment ion produced prior to introduction of $\mathrm{D}_{2} \mathrm{O}$ is then subtracted from this value to obtain the overall $\mathrm{m} / \mathrm{z}$ shift resulting from deuterium incorporation.

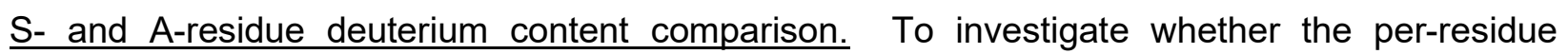
deuterium content reveals differences in relative residue accessibility between the $S$ - and Acontaining peptides, the per-residue deuterium content is here compared. As mentioned above, one comparison that is readily available is that of overall HDX efficiency for these resideus. For the S-containing peptide, on average, the number of deuteriums incorporated for all $\mathrm{S}$ residues is $\sim 9.4(\sim 28.5 \%$ of the 33 total available). For the A-containing peptide, a value of $\sim 19.5 \%$ efficiency ( 3.3 of 17 available sites), on average, is obtained. The higher efficiency could be reflective of the more compact nature of the S-containing peptide as more exchange sites are in closer proximity to charge sites and deuterium incorporation sites as required by the reaction mechanism ${ }^{75}$.

For the reactivity comparisons at the per-residue level, a question arises as to how the evaluation should be performed. For example, the S-containing peptide contains 16 more exchangeable hydrogens due to the side-chain site on the $S$ residues when compared with the A-containing peptide ions. Therefore a direct comparison of per-residue deuterium content may not provide the simplest representation of the relative accessibility. Thus the $\mathrm{S}$ and $\mathrm{A}$ deuterium 
content values are scaled according to their fractional values of the total deuterium incorporated into the neutral residues. $\mathrm{K}$ residues are not included in this analysis because, considering the HDX mechanism, different charge site configurations could contribute to the observed differences. Here, rather the interest is in comparing the average differences in accessibility to different regions of the peptide through the use of the $S$ and $A$ residues.

Figure 2.3 shows the comparison of the deuterium content of the $S$ and $A$ residues on the respective peptide ions. For the A-containing peptide, only four residues show significantly greater accessibility; these are the A4, A5, A19 and A20 residues. For the S-containing peptide, the residues showing significantly increased accessibility are the $S 2, S 9, S 10, S 13, S 15$, and S17 residues. Overall, the results suggest a greater accessibility to middle residues for the Scontaining peptide ion. This could result from increased charge solvation by the $S$ residues as discussed below. Notably, the relative per-residue deuterium uptake values shown in Figure 2.3 exhibit different levels of precision (also shown as independent values in Table S1). This appears to primarily result from variability in determining the peak centers of low abundance fragment ions. For example, fragment ions used to determine the deuterium content for S3 and S18 residues are $\sim 40 \%$ lower in intensity relative to those used for the S13 and S19 residues. That said, the error levels are in line with those reported previously $72,76,80-81$. 


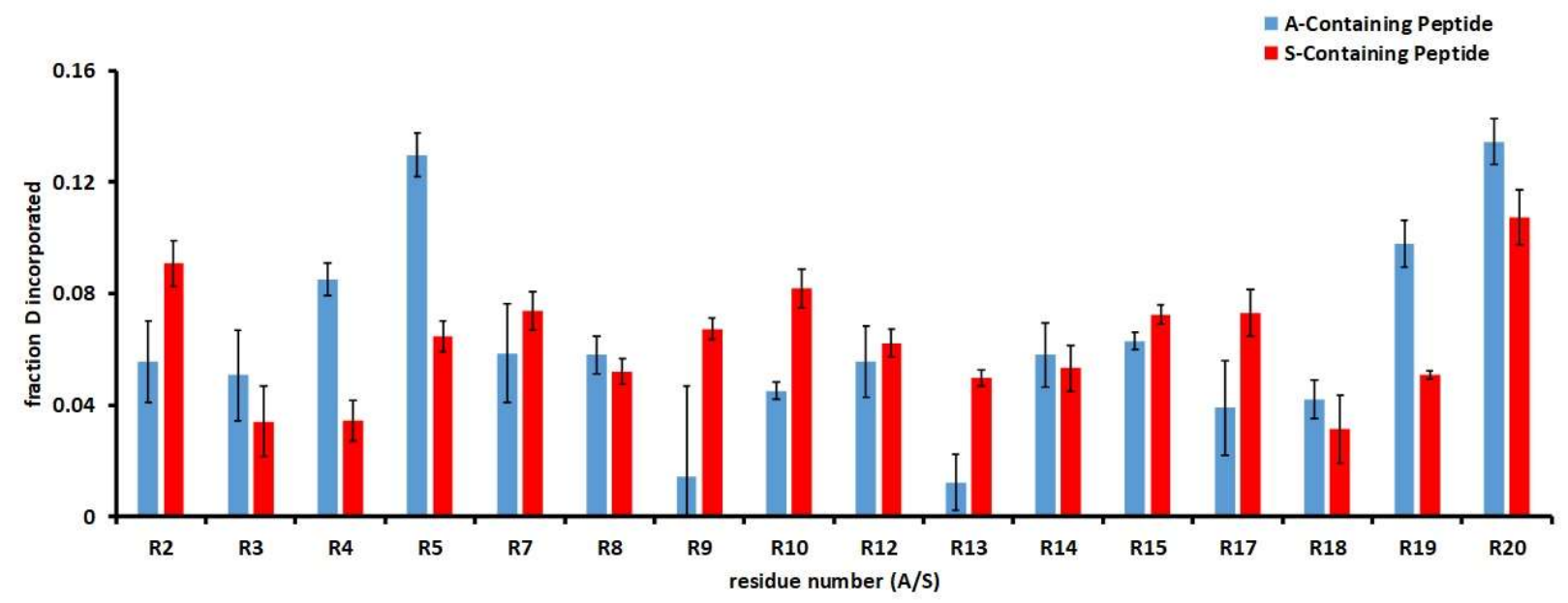

Figure 2. 3 Bar graph showing the fraction of deuterium for $S$ (red bars) and A (blue bars) residues obtained from MS/MS analysis of mobility selected ions.

Fractional values are calculated as the ratio of the deuterium content of the specified residue to the total deuterium content from neutral residues for the respective peptides. Error bars are obtained by propagating the error (one standard deviation) of the individual deuterium uptake values. 
Comparison to candidate structures from MD simulations. The HDX behavior of the Scontaining peptide was further considered using MD simulations. To find the best structural type (or types) exhibiting similar exchange site accessibility to the gas-phase conformer type, simulated annealing was conducted for $[\mathrm{M}+3 \mathrm{H}]^{3+}$ peptide ions using two different charge configurations as well as two different simulation times (see Experimental section). As shown in Figure S3 in the Supplementary Information section (Appendix A), the collision cross sections obtained from MD simulations are, for the most part, significantly smaller than the experimental value. An examination of some of these structures, reveals extremely compact ion conformer types with significant charge solvation of the charge sites by the serine residues as shown in Figure S4 (Supplementary Information section (Appendix A)). Indeed, as an indicator of how the MD simulations captured such charge solvation, consider that only 71 of 8000 structures were observed to have collision cross sections within $\pm 2 \%$ of the experimental value for the compact $[\mathrm{M}+3 \mathrm{H}]^{3+}$ ions.

To consider which of the structural types with matching collision cross sections best represented the experimental data, HDX kinetics modeling was employed (see Experimental section above). Notably, this work does not include the very extensive MD trajectory modeling that was performed previously for the A-containing peptide ${ }^{34-35,76}$. Rather, here the MD simulations are only performed to provide an idea of conformer types that could account for the observed HDX accessibility ${ }^{72}$. From HAS scoring and kinetics modeling of the 71 structures, several conformer types appeared to exhibit significant charge solvation by middle residues. Based on the best ability to match the experimental results (see below), one of these ion structures was selected for discussion. This ion structure is shown in Figure 2.4C. 


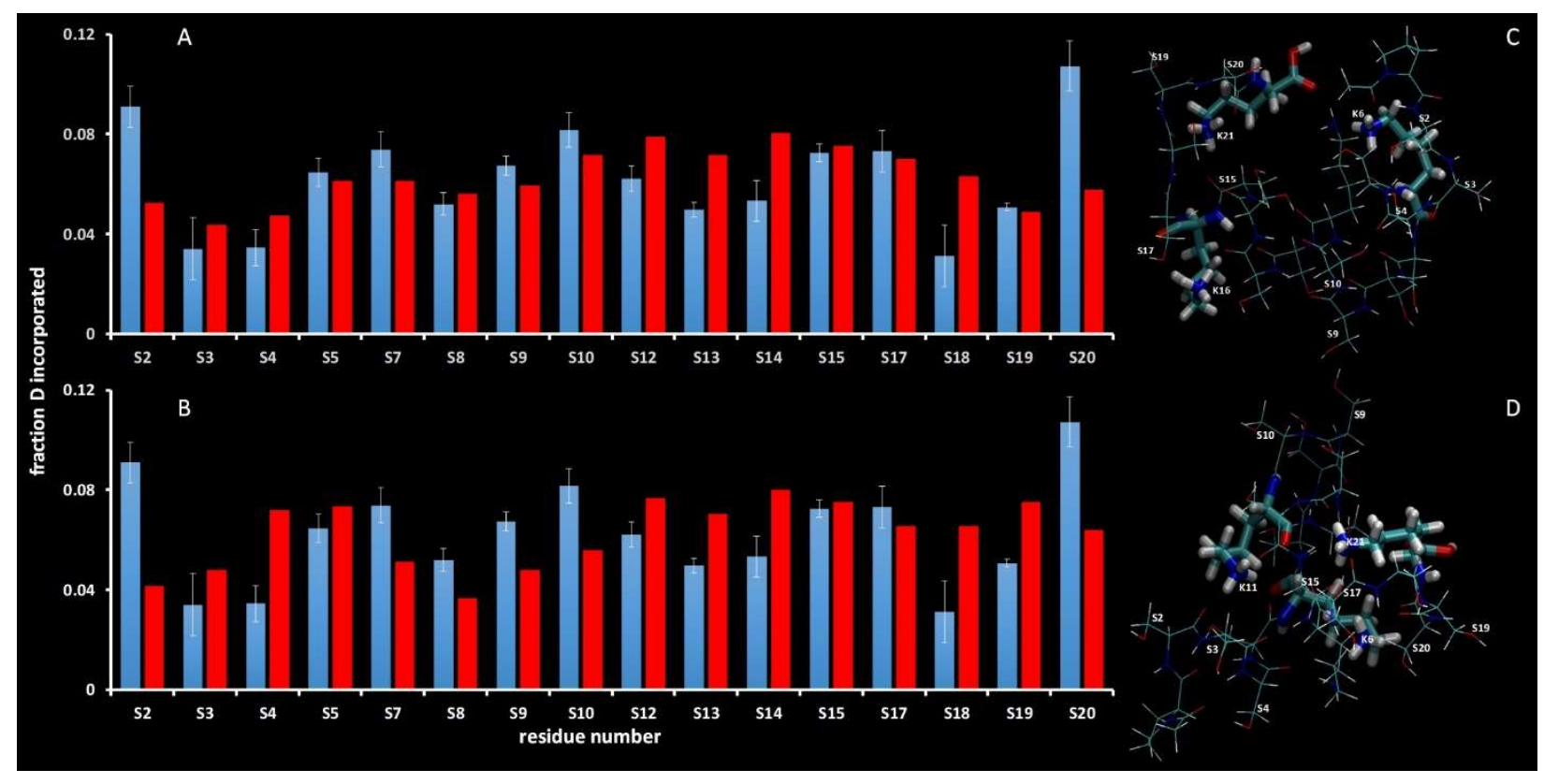

Figure 2. 4 The comparison of the experimental and theoretical fraction deuterium incorporated values.

Panel A shows the comparison of the experimental fraction deuterium incorporated values (blue bars) and the theoretical fraction deuterium incorporated values (red bars) for the ion conformation shown in panel $\mathrm{C}$ of the model peptide Acetyl-PSSSSKSSSSKSSSSKSSSSK. Panel B shows the same comparison for the ion conformation shown in panel D. In panels $C$ and $D$, several residues are labeled to demonstrate their relative accessibility for HDX (see text for details). 
The agreement between experimental and theoretical (HDX kinetics modeling) relative deuterium content is shown as a bar graph in Figure 2.4A. A number of trends are shown to agree between the experimental and theoretical values. Here we note that the theory favors sites that are in relative proximity to backbone carbonyls that are in relative proximity to charge sites. A trend captured by the theory for the first ion conformer is the relatively low levels of uptake for S3 and S4 and an increase in uptake for S5 and S7. This is followed by a slight decrease for S8 and then increases for S9 and S10. The model slightly overestimates the deuterium content for S12 to $\mathrm{S} 14$. This is followed by relatively good agreement between $\mathrm{S} 15$ and S17. The worst agreement is observed at the C-terminal and N-terminal ends for the S2, S18, and S20 residues. Overall, the average percent difference between experiment and theory is observed to be $\sim 27.5 \%$ across all $S$ residues.

Figure 2.4D shows a separate ion conformer for which increased accessibility is observed for the terminal peptide portions relative to middle residues. For example, for this ion conformer there is an increase in estimated deuterium content for the N-terminal residues S3, S4, and S5 with an increase also observed for the C-terminal residues S18, S19, and S20. These increases are offset by decreases in theoretical deuterium content for residues S7 to S14 as well as S17. For this structure, the average percent difference between experiment and theory is $\sim 41.4 \%$.

The differences in the quality of HDX reactivity matching for the ion candidate structures can be described via the HAS scores and the structure images shown in Figure 2.4. For the ion structure providing the best HDX reactivity matching, the charge site assignments are $\mathrm{K} 6, \mathrm{~K} 16$, and $\mathrm{K} 21$ while the charge site assignments for the second ion structure are $\mathrm{K} 6, \mathrm{~K} 11$, and $\mathrm{K} 21$. One noticeable difference in the structures is that the former ion conformer with its charge arrangement exhibits less charge density. Indeed, the relative Coulomb energy of the former ion conformer is only $69 \%$ that of the latter. Thus a more even dispersion of charge throughout 
the ion conformer is observed. For the first ion conformer (Figure 2.4C), the relative increase in theoretical deuterium content would be elevated for the $S 17$ residue due to its hydrogens proximity to the $\mathrm{S} 13(2.9 \AA), \mathrm{S} 14(2.1 \AA)$, and $\mathrm{S} 17(3.6 \AA)$ carbonyls. These carbonyls are highly scored because they are in close proximity to the K21 charge site (all $\leq 1 \AA$ ) and the S13 carbonyl is also within $2.5 \AA$ of the K16 charge site. The S15 residue has a large theoretical deuterium uptake as well due to its hydrogens proximity to the $\mathrm{K} 11$ (1.9 $\AA)$, S12 (3.0 $)$, and S14 (3.1 A) carbonyls. The K11 and S12 carbonyls are scored highly because they are within 3 $\AA$ of the K16 and K21 charge sites. In comparison, only one scored carbonyl is positioned at $\leq 4 \AA$ from the $\mathrm{S} 19$ hydrogens. The increased theoretical deuterium uptake for the $\mathrm{S} 10$ to the S14 sites results from the fact that these residues fall between the $\mathrm{K} 6$ and $\mathrm{K} 16$ charge sites and there is a significant compaction of this portion of the ion such that exchange sites are in close proximity to a greater number of carbonyl sites. For example, the $\mathrm{S} 10$ residue has hydrogens that are within $1.8 \AA$ and $2.9 \AA$ of scored carbonyls on the S4 and S7 residues. Additionally, labile hydrogens are $\leq 4 \AA$ of scored carbonyls on the S8 and S9 residues. These scored carbonyls are in closer proximity to the K6 charge site. Finally, although the S2, S3, and S4 residues are in relative proximity to the $\mathrm{K} 6$ charge site, there is a decreased number of close carbonyl sites. For example, for hydrogens on the $\mathrm{S} 2$ residue, only the $\mathrm{S} 2$ carbonyl is within $\leq 4$ $\AA$.

For the ion conformation presenting decreased agreement in theoretical and experimental deuterium content (Figure 2.4), the decrease at S2 results from the placement of this residue further away from charge sites. This is evident from the fact that the only carbonyl sites within 4 $\AA$ of any hydrogen on the $\mathrm{S} 2$ residue are the $\mathrm{N}$-terminal acetyl group and the $\mathrm{P} 1$ residue and both of these carbonyls are not highly scored due to their distance to charge sites. Decreases in middle residues $S 9$ and $\mathrm{S10}$ can be seen to result from the placement of these residues further away from charge sites and scored carbonyl sites. For example, hydrogens on the S10 
residue only encounter the S8 carbonyl at a distance that is $\leq 4 \AA$. Finally, the increased theoretical deuterium content at the C-terminal residues (S18, S19, and S20) results from the fact that these residues are in close proximity to the $\mathrm{K} 6$ and $\mathrm{K} 21$ charge sites as well as a number of carbonyl sites. For example, the hydrogens on the $S 19$ residue are within $1.5 \AA$ and $2.5 \AA$ of the scored carbonyls on the S18 and S19 residues. These carbonyls are scored highly as they are $0.5 \AA$ and $1.7 \AA$ from the K21 charge site. Additionally, the latter carbonyl is also within $5.7 \AA$ of the $\mathrm{K} 6$ and $\mathrm{K} 11$ residues. As shown in Figure 2.4, the structure exhibiting the decreased HDX reactivity match appears to position the S4 and S5 residues such that they would not exhibit greater accessibility as determined by the HAS approach. However, upon rotation of the ion structure, it becomes apparent that the exchangeable hydrogens on the S4 and S5 residues are near a number of highly scored carbonyl sites as shown in Figure S5 in the Supplementary Information section (Appendix A). Indeed, the hydrogens on these residues are $\leq 2 \AA$ from the $\mathrm{N}$-terminal acetyl group carbonyl and the $\mathrm{P} 1$ carbonyl.

It is instructive to consider the type of ion structure that would be required to result in increased exchange of the S2 and S20 residues as is observed experimentally (Figure 2.4). One consideration is that more compact structures in which the $\mathrm{N}$-terminal and C-terminal ends turn back toward the middle residues would provide increased HAS scores for these residues. Figure S6 in the Supplementary Information section (Appendix A) shows a compact ion structure $\left({ }^{\mathrm{He}} \Omega_{\mathrm{DT}}=363.0 \AA^{2}\right)$ for which HAS scoring and HDX kinetics modeling was performed. The relative deuterium incorporation levels for several residues are also shown in Figure S6 (Appendix A). Notably, the value for the S20 residue is the largest of all residues and that of S2 is among the largest values as well. Additionally, the deuterium incorporation values for the neighboring S4 and S18 residues are among the lowest. These results are similar to what is observed experimentally for $\mathrm{N}$ - and C-terminal residues (Figure 2.3 and Figure 2.4). The high value for the S2 residue results from the fact that a $\mathrm{N}$-terminal bend positions this residue such 
that the $S 2$ backbone hydrogen is in relatively close proximity of the acetyl carbonyl (3.3 $\AA$ ), the P1 carbonyl (3.1 $\AA$ ), and the S2 carbonyl (2.1 $)$. These carbonyl sites also have relatively high scores as they are located near the K6 charge site. The side-chain hydrogen of S2 is located near $(1.7 \AA)$ the $\mathrm{S} 10$ carbonyl which is one of the highest scoring carbonyl sites being near multiple charge sites. In contrast, the backbone hydrogen and the side-chain hydrogen of the S4 residue are not within 3 and $4 \AA$, respectively, of any scored carbonyl. For the $S 20$ residue, hydrogens are in close proximity to the highly scored carbonyls on S19 (3.1 $\AA$ ) and S8 (2.0 $\AA)$. The S18 residue hydrogens are not within $3 \AA$ of any scored carbonyl.

It is necessary to present a note of caution regarding the comparisons between theoretical and experimental deuterium uptake here. Such comparisons are not intended to be definitive as they do not consider ion conformer dynamics or the possible contribution by multiple ion conformers with different charge site configurations. As mentioned above, the failings of the approach are especially obvious in the inability of either ion structure to account for the increased deuterium content of the $S 2$ and $S 20$ residues observed experimentally. The improved matching of HAS scores with experimental values for these residues when using a far too compact ion structure is intriguing. For example, such analysis may suggest that multiple ion conformer types comprise the mobility selection and a portion of these contain bends at either the $\mathrm{N}$-terminal or the C-terminal end with the other end extended (in order to exhibit the correct cross section). That said, the comparisons are useful insofar as they demonstrate conformer types exhibiting specific charge solvation that could account for the observed differences in relative deuterium content of specific peptide regions. Additionally, such comparisons represent the foundational work that will be extended to exhaustive MD simulations that do account for conformer dynamics as well as the presence of different conformer types. In future works, such simulations will be conducted in the gas and solution 
phases as was performed for the A-containing peptide ${ }^{34-35}$ and the results will be compared with regard to the preferred types of structure formed for the different ions.

\section{Conclusions}

IMS-HDX-MS/MS measurements coupled with MD simulations have been used to gain insight into the behavior of $[\mathrm{M}+3 \mathrm{H}]^{3+}$ ions of the model peptide Acetyl-PSSSSKSSSSKSSSSKSSSSK. From the IMS measurements a dominant, compact ion conformer is observed. In comparison to the same ions for the model peptide Acetyl-PAAAAKAAAAKAAAAKAAAAK, the S-containing peptide is observed to be relatively more compact. This may be somewhat expected as CD spectroscopy suggests that the former peptide exhibits more random coil nature while the Acontaining peptide exhibits significantly more a-helical character. In addition to, and likely attributed to, the increased compactness, the S-containing peptide shows increased accessibility of interior residues to gas-phase HDX. MD simulations suggest that ion conformers demonstrating specific, relatively disperse charge solvation by interior residues could account for the observed HDX behavior of the $[\mathrm{M}+3 \mathrm{H}]^{3+}$ ions.

The disparity in hypothetical and experimental deuterium uptake suggests the need for highly extensive MD simulations to better understand the contributions of ion structure dynamics as well as contributions from multiple ion conformers. This limitation was also encountered for the A-containing peptide where exhaustive gas-phase and ESI droplet simulations have been conducted to better fit the experimental data ${ }^{34-35}$. Thus, in order to gain insight into the ion structures resulting from very different solution structures, it will be necessary, in future studies, to conduct these same exhaustive analyses where a convergence between gas-phase ion structures and those emerging from ESI droplets is obtained. With these structures it will be possible to compare the major differences in structures arising from the S- and A-containing peptides. The comparison of such predominant structures could provide information about the 
degree of preservation of secondary structure for these smaller biomolecules and could thus help with our understanding of the process of gas-phase ion structure establishment.

\section{Acknowledgments}

We are grateful for financial support from the National Institutes of Health (R01GM114494).

\section{References}

1. Pauling, L.; Corey, R. B., TWO HYDROGEN-BONDED SPIRAL CONFIGURATIONS OF THE POLYPEPTIDE CHAIN. J Am Chem Soc 1950, 72 (11), 5349-5349.

2. Pace, C. N.; Scholtz, J. M., A helix propensity scale based on experimental studies of peptides and proteins. Biophysical Journal 1998, 75 (1), 422-427.

3. Davies, D. R., A CORRELATION BETWEEN AMINO ACID COMPOSITION AND PROTEIN STRUCTURE. Journal of molecular biology 1964, 9, 605-609.

4. Guzzo, A. V., The Influence of Amino Acid Sequence on Protein Structure. Biophysical Journal 1965, 5 (6), 809-822.

5. Prothero, J. W., Correlation between the distribution of amino acids and alpha helices. Biophysical Journal 1966, 6 (3), 367-370.

6. Cook, D. A., The relation between amino acid sequence and protein conformation. Journal of Molecular Biology 1967, 29 (1), 167-171.

7. Ptitsyn, O. B., Statistical analysis of the distribution of amino acid residues among helical and non-helical regions in globular proteins. Journal of Molecular Biology 1969, 42 (3), 501-510.

8. Chou, P. Y.; Fasman, G. D., Conformational parameters for amino acids in helical, $\beta$-sheet, and random coil regions calculated from proteins. Biochemistry 1974, 13 (2), 211-222.

9. Defay, T.; Cohen, F. E., Evaluation of current techniques for ab initio protein structure prediction. Proteins 1995, 23 (3), 431-45.

10. Frishman, D.; Argos, P., The future of protein secondary structure prediction accuracy. Folding and Design 1997, 2 (3), 159-162.

11. Creamer, T. P.; Rose, G. D., Alpha-helix-forming propensities in peptides and proteins. Proteins 1994, 19 (2), 85-97.

12. Horovitz, A.; Matthews, J. M.; Fersht, A. R., Alpha-helix stability in proteins. II. Factors that influence stability at an internal position. J Mol Biol 1992, 227 (2), 560-8.

13. Krause, E.; Bienert, M.; Schmieder, P.; Wenschuh, H., The Helix-Destabilizing Propensity Scale of d-Amino Acids: The Influence of Side Chain Steric Effects. J Am Chem Soc 2000, 122 (20), 4865-4870.

14. Towse, C.-L.; Hopping, G.; Vulovic, I.; Daggett, V., Nature versus design: the conformational propensities of d-amino acids and the importance of side chain chirality. Protein Engineering, Design and Selection 2014, 27 (11), 447-455.

15. Manjasetty, B. A.; Büssow, K.; Panjikar, S.; Turnbull, A. P., Current methods in structural proteomics and its applications in biological sciences. 3 Biotech 2012, 2 (2), 89-113.

16. Berman, H. M.; Westbrook, J.; Feng, Z.; Gilliland, G.; Bhat, T. N.; Weissig, H.; Shindyalov, I. N.; Bourne, P. E., The Protein Data Bank. Nucleic Acids Research 2000, 28 (1), 235-242. 
17. Petrey, D.; Honig, B., Protein structure prediction: inroads to biology. Molecular cell 2005, 20 (6), 811-9.

18. Qu, X. T.; Swanson, R.; Day, R.; Tsai, J., A Guide to Template Based Structure Prediction. Current Protein \& Peptide Science 2009, 10 (3), 270-285.

19. Forrest, L. R.; Tang, C. L.; Honig, B., On the Accuracy of Homology Modeling and Sequence Alignment Methods Applied to Membrane Proteins. Biophysical Journal 2006, 91 (2), 508-517.

20. Dorn, M.; MB, E. S.; Buriol, L. S.; Lamb, L. C., Three-dimensional protein structure prediction: Methods and computational strategies. Computational biology and chemistry 2014, 53pb, 251-276.

21. Li, B.; Fooksa, M.; Heinze, S.; Meiler, J., Finding the needle in the haystack: towards solving the protein-folding problem computationally. Critical Reviews in Biochemistry and Molecular Biology 2018, 53 (1), 1-28.

22. Tian, X. Y.; Chen, D. Z.; Gao, J., An Overview on Protein Fold Classification via Machine Learning Approach. Current Proteomics 2018, 15 (2), 85-98.

23. Almeida, J. G.; Preto, A. J.; Koukos, P. I.; Bonvin, A.; Moreira, I. S., Membrane proteins structures: A review on computational modeling tools. Biochimica Et Biophysica Acta-Biomembranes 2017, 1859 (10), 2021-2039.

24. Uversky, V. N., What does it mean to be natively unfolded? European Journal of Biochemistry 2002, 269 (1), 2-12.

25. Chatterjee, A.; Kumar, A.; Chugh, J.; Srivastava, S.; Bhavesh, N. S.; Hosur, R. V., NMR of unfolded proteins. Journal of Chemical Sciences 2005, 117 (1), 3-21.

26. Fink, A. L., Natively unfolded proteins. Current Opinion in Structural Biology 2005, 15 (1), 35-41.

27. Bracken, C.; lakoucheva, L. M.; Rorner, P. R.; Dunker, A. K., Combining prediction, computation and experiment for the characterization of protein disorder. Current Opinion in Structural Biology 2004, 14 (5), 570-576.

28. Meng, F. C.; Uversky, V. N.; Kurgan, L., Comprehensive review of methods for prediction of intrinsic disorder and its molecular functions. Cellular and Molecular Life Sciences 2017, 74 (17), 30693090.

29. Hicks, R. P., Recent advances in NMR: expanding its role in rational drug design. Current medicinal chemistry 2001, 8 (6), 627-50.

30. Pierson, N. A.; Chen, L.; Valentine, S. J.; Russell, D. H.; Clemmer, D. E., Number of solution states of bradykinin from ion mobility and mass spectrometry measurements. J Am Chem Soc 2011, 133 (35), 13810-3.

31. Pierson, N. A.; Valentine, S. J.; Clemmer, D. E., Evidence for a Quasi-Equilibrium Distribution of States for Bradykinin $\mathrm{M}+3 \mathrm{H}(3+)$ lons in the Gas Phase. Journal of Physical Chemistry B 2010, 114 (23), 7777-7783.

32. Shi, H. L.; Pierson, N. A.; Valentine, S. J.; Clemmer, D. E., Conformation Types of Ubiquitin M+8H (8+) lons from Water:Methanol Solutions: Evidence for the $\mathrm{N}$ and A States in Aqueous Solution. Journal of Physical Chemistry B 2012, 116 (10), 3344-3352.

33. Khakinejad, M.; Ghassabi Kondalaji, S.; Tafreshian, A.; Valentine, S. J., Comprehensive Gas-Phase Peptide Ion Structure Studies Using Ion Mobility Techniques: Part 2. Gas-Phase Hydrogen/Deuterium Exchange for Ion Population Estimation. Journal of The American Society for Mass Spectrometry 2017, 111.

34. Ghassabi Kondalaji, S.; Khakinejad, M.; Tafreshian, A.; S, J. V., Comprehensive Peptide Ion Structure Studies Using Ion Mobility Techniques: Part 1. An Advanced Protocol for Molecular Dynamics Simulations and Collision Cross-Section Calculation. J Am Soc Mass Spectrom 2017, 28 (5), 947-959.

35. Ghassabi Kondalaji, S. K., M.; Valentine, S. J., Comprehensive Peptide Ion Structure Studies Using Ion Mobility Techniques: Part 3. Relating Solution-phase to Gas-phase Structures. Journal of the American Society for Mass Spectrometry (accepted) 2018. 
36. Chakrabartty, A.; Kortemme, T.; Baldwin, R. L., Helix propensities of the amino acids measured in alanine-based peptides without helix-stabilizing side-chain interactions. Protein Science : A Publication of the Protein Society 1994, 3 (5), 843-852.

37. Palencar, P.; Bleha, T., Molecular dynamics simulations of the folding of poly(alanine) peptides. Journal of molecular modeling 2011, 17 (9), 2367-74.

38. Hudgins, R. R.; Ratner, M. A.; Jarrold, M. F., Design of helices that are stable in vacuo. J Am Chem Soc 1998, 120 (49), 12974-12975.

39. Straus, R. N.; Jockusch, R. A., Probing the Gaseous Structure of a beta-Hairpin Peptide with H/D Exchange and Electron Capture Dissociation. J Am Soc Mass Spectrom 2017, 28 (2), 358-369.

40. Pan, J. X.; Heath, B. L.; Jockusch, R. A.; Konermann, L., Structural Interrogation of Electrosprayed Peptide Ions by Gas-Phase H/D Exchange and Electron Capture Dissociation Mass Spectrometry. Anal. Chem. 2012, 84 (1), 373-378.

41. Ballesteros, J. A.; Deupi, X.; Olivella, M.; Haaksma, E. E.; Pardo, L., Serine and threonine residues bend alpha-helices in the chi(1) = g(-) conformation. Biophysical Journal 2000, 79 (5), 2754-2760.

42. Imai, K.; Mitaku, S., Mechanisms of secondary structure breakers in soluble proteins. Biophysics 2005, 1, 55-65.

43. Konermann, L.; Ahadi, E.; Rodriguez, A. D.; Vahidi, S., Unraveling the Mechanism of Electrospray Ionization. Anal. Chem. 2013, 85 (1), 2-9.

44. Kim, D.; Wagner, N.; Wooding, K.; Clemmer, D. E.; Russell, D. H., Ions from Solution to the Gas Phase: A Molecular Dynamics Simulation of the Structural Evolution of Substance $\mathrm{P}$ during Desolvation of Charged Nanodroplets Generated by Electrospray lonization. J Am Chem Soc 2017, 139 (8), 29812988.

45. Glaskin, R. S.; Khatri, K.; Wang, Q.; Zaia, J.; Costello, C. E., Construction of a Database of Collision Cross Section Values for Glycopeptides, Glycans, and Peptides Determined by IM-MS. Anal. Chem. 2017, 89 (8), 4452-4460.

46. Soper-Hopper, M. T.; Petrov, A. S.; Howard, J. N.; Yu, S. S.; Forsythe, J. G.; Grover, M. A.; Fernandez, F. M., Collision cross section predictions using 2-dimensional molecular descriptors. Chemical Communications 2017, 53 (54), 7624-7627.

47. Zheng, X. Y.; Deng, L. L.; Baker, E. S.; Ibrahim, Y. M.; Petyuk, V. A.; Smith, R. D., Distinguishing Dand L-aspartic and isoaspartic acids in amyloid beta peptides with ultrahigh resolution ion mobility spectrometry. Chemical Communications 2017, 53 (56), 7913-7916.

48. Daly, S.; MacAleese, L.; Dugourd, P.; Chirot, F., Combining Structural Probes in the Gas Phase Ion Mobility-Resolved Action-FRET. Journal of the American Society for Mass Spectrometry 2018, 29 (1), 133-139.

49. Benigni, P.; Porter, J.; Ridgeway, M. E.; Park, M. A.; Fernandez-Lima, F., Increasing Analytical Separation and Duty Cycle with Nonlinear Analytical Mobility Scan Functions in TIMS-FT-ICR MS. Anal. Chem. 2018, 90 (4), 2446-2450.

50. Stocks, B. B.; Melanson, J. E., In-Source Reduction of Disulfide-Bonded Peptides Monitored by Ion Mobility Mass Spectrometry. Journal of the American Society for Mass Spectrometry 2018, 29 (4), 742-751.

51. Heo, C. E.; Choi, T. S.; Kim, H. I., Competitive homo- and hetero- self-assembly of amyloid-beta 142 and 1-40 in the early stage of fibrillation. International Journal of Mass Spectrometry 2018, 428, 1521.

52. Fouque, K. J. D.; Garabedian, A.; Porter, J.; Baird, M.; Pang, X. Q.; Williams, T. D.; Li, L. J.; Shvartsburg, A.; Fernandez-Lima, F., Fast and Effective Ion Mobility-Mass Spectrometry Separation of DAmino-Acid-Containing Peptides. Anal. Chem. 2017, 89 (21), 11787-11794. 
53. Lee, J. W.; Lee, H. H. L.; Davidson, K. L.; Bush, M. F.; Kim, H. I., Structural characterization of small molecular ions by ion mobility mass spectrometry in nitrogen drift gas: improving the accuracy of trajectory method calculations. Analyst 2018, 143 (8), 1786-1796.

54. Clowers, B. H.; Hill, H. H., Mass analysis of mobility-selected ion populations using dual gate, ion mobility, quadrupole ion trap mass spectrometry. Anal. Chem. 2005, 77 (18), 5877-5885.

55. Zucker, S. M.; Lee, S.; Webber, N.; Valentine, S. J.; Reilly, J. P.; Clemmer, D. E., An Ion Mobility/Ion Trap/Photodissociation Instrument for Characterization of Ion Structure. Journal of the American Society for Mass Spectrometry 2011, 22 (9), 1477-1485.

56. Khakinejad, M.; Kondalaji, S. G.; Maleki, H.; Arndt, J. R.; Donohoe, G. C.; Valentine, S. J., Combining ion mobility spectrometry with hydrogen-deuterium exchange and top-down MS for peptide ion structure analysis. J Am Soc Mass Spectrom 2014, 25 (12), 2103-15.

57. Donohoe, G. C.; Maleki, H.; Arndt, J. R.; Khakinejad, M.; Yi, J. H.; McBride, C.; Nurkiewicz, T. R.; Valentine, S. J., A New Ion Mobility-Linear Ion Trap Instrument for Complex Mixture Analysis. Anal. Chem. 2014, 86 (16), 8121-8128.

58. Wood, T. D.; Chorush, R. A.; Wampler, F. M.; Little, D. P.; Oconnor, P. B.; McLafferty, F. W., GASPHASE FOLDING AND UNFOLDING OF CYTOCHROME-C CATIONS. Proc. Natl. Acad. Sci. U. S. A. 1995, 92 (7), 2451-2454.

59. Suckau, D.; Shi, Y.; Beu, S. C.; Senko, M. W.; Quinn, J. P.; Wampler, F. M.; McLafferty, F. W., COEXISTING STABLE CONFORMATIONS OF GASEOUS PROTEIN IONS. Proc. Natl. Acad. Sci. U. S. A. 1993, 90 (3), 790-793.

60. Valentine, S. J.; Clemmer, D. E., H/D exchange levels of shape-resolved cytochrome c conformers in the gas phase. J Am Chem Soc 1997, 119 (15), 3558-3566.

61. Wyttenbach, T.; Bowers, M. T., Gas phase conformations of biological molecules: The hydrogen/deuterium exchange mechanism. Journal of the American Society for Mass Spectrometry 1999, $10(1), 9-14$.

62. Freitas, M. A.; Hendrickson, C. L.; Emmett, M. R.; Marshall, A. G., Gas-phase bovine ubiquitin cation conformations resolved by gas-phase hydrogen/deuterium exchange rate and extent. International Journal of Mass Spectrometry 1999, 185, 565-575.

63. Chipuk, J. E.; Brodbelt, J. S., Investigation of the gas-phase hydrogen/deuterium exchange behavior of aromatic dicarboxylic acids in a quadrupole ion trap. International Journal of Mass Spectrometry 2007, 267 (1-3), 98-108.

64. Zhang, J. M.; Brodbelt, J. S., Gas-phase hydrogen/deuterium exchange and conformations of deprotonated flavonoids and gas-phase acidities of flavonoids. J Am Chem Soc 2004, 126 (18), 59065919.

65. Gabelica, V.; Rosu, F.; Witt, M.; Baykut, G.; De Pauw, E., Fast gas-phase hydrogen/deuterium exchange observed for a DNA G-quadruplex. Rapid communications in mass spectrometry : RCM 2005, 19 (2), 201-8.

66. Mistarz, U. H.; Brown, J. M.; Haselmann, K. F.; Rand, K. D., Simple Setup for Gas-Phase H/D Exchange Mass Spectrometry Coupled to Electron Transfer Dissociation and Ion Mobility for Analysis of Polypeptide Structure on a Liquid Chromatographic Time Scale. Anal. Chem. 2014, 86 (23), 11868-11876.

67. Rand, K. D.; Zehl, M.; Jensen, O. N.; Jorgensen, T. J., Loss of ammonia during electron-transfer dissociation of deuterated peptides as an inherent gauge of gas-phase hydrogen scrambling. Anal Chem 2010, 82 (23), 9755-62.

68. Rand, K. D.; Adams, C. M.; Zubarev, R. A.; Jorgensen, T. J. D., Electron capture dissociation proceeds with a low degree of intramolecular migration of peptide amide hydrogens. J Am Chem Soc 2008, 130 (4), 1341-1349. 
69. Syka, J. E. P.; Coon, J. J.; Schroeder, M. J.; Shabanowitz, J.; Hunt, D. F., Peptide and protein sequence analysis by electron transfer dissociation mass spectrometry. Proc. Natl. Acad. Sci. U. S. A. 2004, 101 (26), 9528-9533.

70. Khakinejad, M.; Kondalaji, S. G.; Tafreshian, A.; Valentine, S. J., Gas-Phase Hydrogen-Deuterium Exchange Labeling of Select Peptide Ion Conformer Types: a Per-Residue Kinetics Analysis. J Am Soc Mass Spectrom 2015.

71. Donohoe, G. C.; Arndt, J. R.; Valentine, S. J., Online deuterium hydrogen exchange and protein digestion coupled with ion mobility spectrometry and tandem mass spectrometry. Anal Chem 2015, 87 (10), 5247-54.

72. Khakinejad, M.; Kondalaji, S. G.; Tafreshian, A.; Valentine, S. J., Gas-Phase Hydrogen-Deuterium Exchange Labeling of Select Peptide Ion Conformer Types: a Per-Residue Kinetics Analysis. J Am Soc Mass Spectrom 2015, 26 (7), 1115-27.

73. Jarrold Research Group, I. U. MOBCAL - A Program to Calculate Mobilities. http://www.indiana.edu/ nano/software.html (accessed April 17).

74. Mesleh, M. F.; Hunter, J. M.; Shvartsburg, A. A.; Schatz, G. C.; Jarrold, M. F., Structural Information from lon Mobility Measurements: Effects of the Long-Range Potential. The Journal of Physical Chemistry 1996, 100 (40), 16082-16086.

75. Campbell, S.; Rodgers, M. T.; Marzluff, E. M.; Beauchamp, J. L., Deuterium exchange reactions as a probe of biomolecule structure. Fundamental studies of cas phase $H / D$ exchange reactions of protonated glycine oligomers with D2O, CD3OD, CD3CO2D, and ND3. J Am Chem Soc 1995, 117 (51), 12840-12854.

76. Khakinejad, M.; Ghassabi Kondalaji, S.; Tafreshian, A.; Valentine, S. J., Comprehensive Gas-Phase Peptide Ion Structure Studies Using Ion Mobility Techniques: Part 2. Gas-Phase Hydrogen/Deuterium Exchange for Ion Population Estimation. J Am Soc Mass Spectrom 2017, 28 (5), 960-970.

77. Mason, E. A. M., E. W., Transport Properties of lons in Gases. Wiley: New York, 1988.

78. Wyttenbach, T.; Bowers, M. T., Structural Stability from Solution to the Gas Phase: Native Solution Structure of Ubiquitin Survives Analysis in a Solvent-Free Ion Mobility-Mass Spectrometry Environment. Journal of Physical Chemistry B 2011, 115 (42), 12266-12275.

79. Seo, J.; Hoffmann, W.; Warnke, S.; Bowers, M. T.; Pagel, K.; von Helden, G., Retention of Native Protein Structures in the Absence of Solvent: A Coupled Ion Mobility and Spectroscopic Study. Angewandte Chemie-International Edition 2016, 55 (45), 14173-14176.

80. Rand, K. D.; Pringle, S. D.; Morris, M.; Brown, J. M., Site-Specific Analysis of Gas-Phase Hydrogen/Deuterium Exchange of Peptides and Proteins by Electron Transfer Dissociation. Anal. Chem. 2012, 84 (4), 1931-1940.

81. Rand, K. D.; Pringle, S. D.; Murphy, J. P., 3rd; Fadgen, K. E.; Brown, J.; Engen, J. R., Gas-phase hydrogen/deuterium exchange in a traveling wave ion guide for the examination of protein conformations. Anal Chem 2009, 81 (24), 10019-28. 


\section{Investigating the interactions of the first 17 amino acid residues of Huntingtin with lipid vesicles using mass spectrometry and molecular dynamics}

Reproduced with permission from the Journal of Mass Spectrometry: Investigating the interactions of the first 17 amino acid residues of Huntingtin with lipid vesicles using mass spectrometry and molecular dynamics. Kiani Karanji, A., Beasley, M., Sharif, D., Ranjbaran A., Legleiter, J. and Valentine, S. J. Journal of Mass Spectrometry 55 (1), e4470. Copyright ( 2019 John Wiley \& Sons, Ltd.

\section{Introduction}

The majority of cell membrane volume in biological systems is occupied by lipids and proteins. The interactions between such components within complex systems have been studied for years ${ }^{1-5}$. Protein/peptide interaction with the membrane at its surface is an important topic in many fields of study including drug delivery, cell-cell recognition, signal transduction, as well as the study of disease processes ${ }^{6-16}$.

Peptides that interact with the plasma membrane through processes of traversal, fusion, or residing directly at the membrane surface interface are termed membrane active ${ }^{17}$. It has been suggested that the manner in which the peptide locates within the membrane is highly dependent on the membrane lipid composition ${ }^{18}$. Broadly categorized, the interactions have been described as the "barrel-stave" and "carpet" mechanisms ${ }^{17}$. The former process involves the insertion of the peptide into the membrane core while the latter involves binding at the surface primarily through interactions with the polar head groups.

Recently a special type of peptide-membrane interaction has come under increased examination. This involves peptides that form amphipathic helices. In general amphipathic helices are polypeptide regions that form a helix upon interacting with hydrophilic/hydrophobic interfaces. ${ }^{19} \mathrm{~A}$ characteristic of such protein regions is the regular incorporation of non-polar amino acid residues at every third for fourth position. This allows the helix to form in which the 
non-polar face interacts with the lipid constituents of the membrane while the polar face is positioned in the aqueous environment. This anchors the protein to the membrane and orients the helical region parallel to the surface ${ }^{19}$.

Huntington's disease (HD) is one of several neurological conditions characterized by Huntingtin protein (htt) fibril formation in brain tissue. Fibril formation is caused by a polyglutamine (polyQ) expansion occurring in the $\mathrm{N}$-terminal region of the htt ${ }^{20}$. Studies have shown that the first 17 amino acid residues ( $\mathrm{Nt17}$ ) as well as a downstream proline-rich region of htt play important roles in protein stability as well as sub-cellular localization ${ }^{21-25}$. The Nt17 peptide is suggested to play an important role in fibril formation ${ }^{26}$ in which the self-association of the peptide favorably positions the expanded polyQ tracts for aggregation. The subcellular location of htt, necessitates its interaction with the lipid membrane bilayer and is mediated by posttranslational modifications of $\mathrm{Nt17}$ residues ${ }^{27-29}$. Additionally, the interaction of $\mathrm{Nt17}$ with the lipid membrane bilayer has been shown to be an important factor in protein aggregation in HD ${ }^{14,30-32}$ where Nt17 can participate in lipid-binding as well as protein-protein interactions.

The location of hydrophobic residues (L4, L7, A10/F11, L14, and F17) within the primary sequence of the Nt17 peptide suggests a propensity to form an amphipathic helix at the lipid membrane surface. This is supported by recent solid-state NMR studies which suggest that the peptide lies horizontally along the membrane surface ${ }^{33}$. A recent study by Mousseau et al. examined effects of the Nt17 peptide on a phospholipid bilayer ${ }^{34}$. The per-residue helix propensity in the presence of the POPC lipid bilayer was compared with the NMR structure. Overall general agreement was achieved for the two models; however, the MD study suggested that residue numbers 1-4 are more ordered because they are less accessible to the solvent.

Here we present studies of the interaction of Nt17 with the different lipid bilayer systems [1,2-dioleoyl-sn-glycero-3-phosphocholine ～(DOPC), 1-palmitoyl-2-oleoyl-glycero-3phosphocholine (POPC) and 1-palmitoyl-2-oleoyl-sn-glycero-3 phosphoethanolamine (POPE)] 
using native mass spectrometry (MS) ${ }^{35}$ and molecular dynamics (MD) simulations. MS reveals that Nt17 has a different propensity for interaction with vesicles of different lipid compositions. The data suggest that the level of peptide interaction is DOPC $>$ POPC $>$ POPE. Modeling these peptide-lipid systems using MD simulations also supports this interpretation of the MS data and helps to provide a molecular view of the tendency of the peptide to form non-specific interactions with lipids.

In general, the combined study presented here is representative of a growing body of work that utilizes native MS with a goal of elucidating protein interactions with lipid membranes $^{36-40}$ as well as the effect of such interactions upon biomolecular structure ${ }^{41}$. Additionally, the work becomes part of a larger body of studies aimed at determining the mechanisms of aggregation for pathologically-relevant proteins. ${ }^{26,42-52} 53$

\section{Experimental}

Reagents. The Nt17 peptide (>90\% purity) was synthesized by GenScript (Piscataway, NJ, USA) and used without further purification. The peptide sequence is: MATLEKLMKAFESLKSF. The peptide was dissolved in ACS/USP absolute ethanol (Pharmco, Brookfield, CT) at a concentration of $1 \mathrm{mg} / \mathrm{mL}$ to make stock solution. The powder lipids $(1,2-$ dioleoyl-sn-glycero-3-phosphocholine (DOPC), 1-palmitoyl-2-oleoyl-glycero-3-phosphocholine (POPC) and 1-palmitoyl-2-oleoyl-sn-glycero-3 phosphoethanolamine (POPE)) were obtained from Avanti Polar Lipids (Alabaster, $\mathrm{AL}$ ) and dissolved in HPLC grade chloroform (Fisher Scientific, Fair Lawn, NJ) to yield a $10 \mathrm{mg} / \mathrm{mL}$ stock solution for each lipid.

Vesicle Capture-Freeze-Drying (VCFD). Details about sample preparation using the vesicle production method are provided elsewhere ${ }^{41}$. Briefly, $100 \mu \mathrm{L}$ of the stock solution $(\sim 1200 \mu \mathrm{M})$ for each lipid were dried using a speedvac (Thermo Scientific Speedvac) 
maintained under nitrogen gas flow. The dried samples were rehydrated in $1 \mathrm{ml}$ of HPLC grade water with $10 \mathrm{mM}$ ammonium acetate buffer and sonicated for 30 minutes. Then, the samples were subjected to 10 freeze-thaw cycles using liquid nitrogen and a $\sim 50{ }^{\circ} \mathrm{C}$ water bath. Subsequently, $100 \mu \mathrm{L}$ of the stock solution of the $\mathrm{Nt17}$ peptide $(\sim 100 \mu \mathrm{M})$ was added to each lipid solution. For all samples, lipids were maintained at a final concentration that was $\sim 10$ times greater than the Nt17 peptide. The peptide/lipid complex solutions were stored over night at 37 ${ }^{\circ} \mathrm{C}$ for equilibration. Notably, a limitation of this study is that the physiological concentration of Nt17-containing protein is largely unknown although it has been estimated to be $0.15 \mu \mathrm{M}$ in the cytosol of neural cells ${ }^{54}$. Experiments here were designed with higher peptide concentrations $(10 \mu \mathrm{M})$ to enhance the level of peptide-membrane interactions as well as to abbreviate the timescale of such interactions as well as peptide-peptide interactions. Notably this approach is used to study htt aggregation using $\mathrm{THT}^{55}$ and PDA assays ${ }^{56}$ and to perform CD spectroscopy ${ }^{14}$ and $\mathrm{AFM}^{57}$ and $\mathrm{MS}^{38}$ measurements.

Electrospray-Mass Spectrometry (ESI-MS). All of the experiments were carried out using a Q Exactive Hybrid quadruple Orbitrap mass spectrometer (Thermo Fisher, San Jose, CA). The experiments were conducted using the commercial HESI source available with the MS instrument. For both ionization sources MS spectra were recorded in positive ion mode over a mass-to-charge $(\mathrm{m} / \mathrm{z})$ range of 500 to 4000 . The samples were infused at a rate of $10 \mu \mathrm{L} / \mathrm{min}$ using a heated syringe and infusion line to ensure that the POPE vesicles did not undergo a phase transition. The needle was biased at $3,500 \mathrm{~V}$ relative to the instrument inlet. The instrument parameters used for for $\mathrm{ESI}$ were: $450{ }^{\circ} \mathrm{C}$ for the capillary inlet temperature, $80 \mathrm{~V}$ for the S-lens assembly, $400 \mathrm{~ms}$ for the maximum injecting time, $1 \times 10^{6}$ for the AGC, and 70,000 for the MS resolution. Each spectrum was recorded in triplicate for 180 seconds each to ensure data collection reproducibility. To analyze the MS data, the Xcalibur 2.2 software suite (Thermo Scientific) was used. 
A limited number of experiments were performed on a linear ion trap mass spectrometer (LTQ, Thermo Fisher, San Jose, CA) to examine product ions upon isolation of lipidated species (see below). For these experiments, the commercial HESI source was utilized and mass spectra were recorded in positive ion mode using a $\mathrm{m} / \mathrm{z}$ range of 900 to $2000 \mathrm{~m} / \mathrm{z}$ ). The same flow rate and needle bias parameters were used for these experiments. Precursor ions were isolated using a $10 \mathrm{~m} / \mathrm{z}$ window and no collisional activation was employed.

Molecular Dynamics (MD) Simulations. For MD studies, two types of models were used. The first involved initiation with a linear structure to represent the initial interaction between the peptide and the membrane surface. Because full helix formation at the surface of the membrane appeared to be an unreachable event because of the long timescale, MD simulations were also performed in which the peptide was forced to be helical and placed directly above the membrane. Notably the NMR structure suggests that it is helical at the surface $^{33}$. For the latter simulations, the goal was to determine the degree of amphipathic helix interactions with the different lipid membranes.

For the initial random coil simulation of the Nt17 peptide, the fully linear structure was built in Avogadro ${ }^{58}$ and subjected to $10 \mathrm{~ns}$ of simulation in pure water. The output was used as an input file for the Nt17-lipid bilayer simulation. For the helix model, the Nt17 peptide pdb structure (2LD2) was obtained from the protein data bank website ${ }^{33}$ and used as the initial input file for the MD simulations. The CHARMM-GUI server (www.charmm-gui.org) was used to create the lipid bilayer system ${ }^{59}$. The peptide was placed above the bilayer in the $z$ axis and solvated (explicit model) with water. All the simulations were performed in triplicate. The M1 residue at the $\mathrm{N}$-terminus as well as the lysine residues $(\mathrm{K} 6, \mathrm{~K} 9, \mathrm{~K} 15)$ were protonated. The carboxylic acid of F17 at the C-terminus and the two glutamic acid residues (E5, E12) were deprotonated. This provided a net charge of +1 for the modeled peptides. The nano-scale molecular dynamics (NAMD 2.13) software ${ }^{60}$ was utilized to perform minimization and 
equilibration of the system based on the CHARMM-GUI protocol and CHARMM c36 force field for lipids and proteins ${ }^{61}$. The protocol consists of 1950 ps minimization and equilibration process in which restraints from the peptide backbone, lipids, water and ion molecules are gradually removed in six steps ${ }^{62}$. Parameters for all-atom MD simulations using the periodic boundary condition were: NPT ensemble, 2 fs for the timestep, $310 \mathrm{~K}$ for the temperate and 12 $\AA$ for the cutoff. The visual MD (VMD) suite was used to visualize and analyze the simulation data. The GridMAT-MD script was used to calculate the theoretical area per lipid ${ }^{63}$.

\section{Results and Discussion}

Peptide-lipid interactions examined with native MS. Studies have shown that the presence of the Nt17 flanking sequence in the htt protein is important for membrane interactions $14,21,30$. This association has been suggested to serve to anchor huntingtin protein (htt) to the membrane ${ }^{64}$. To investigate the peptide interactions with DOPC lipid, mass spectra were recorded for the sample in which the peptide was incubated with the DOPC vesicles. For these studies, experiments were conducted in duplicate.

Figure 3.1 shows a typical mass spectrum for the DOPC-peptide system. ESI produces ions of several different charge state for the Nt17-lipid system. In the mass spectrum, the doubly-charged monomer peptide ions $\left([\mathrm{M}+2 \mathrm{H}]^{2+}\right.$ at $\left.m / z \sim 988\right)$ predominate. Dimer, trimer and tetramer ions for the $\mathrm{Nt17}$ peptide are also observed at $m / z \sim 1317,1482,1580$, respectively. These species are of significantly lower abundance (Figure 3.1). 


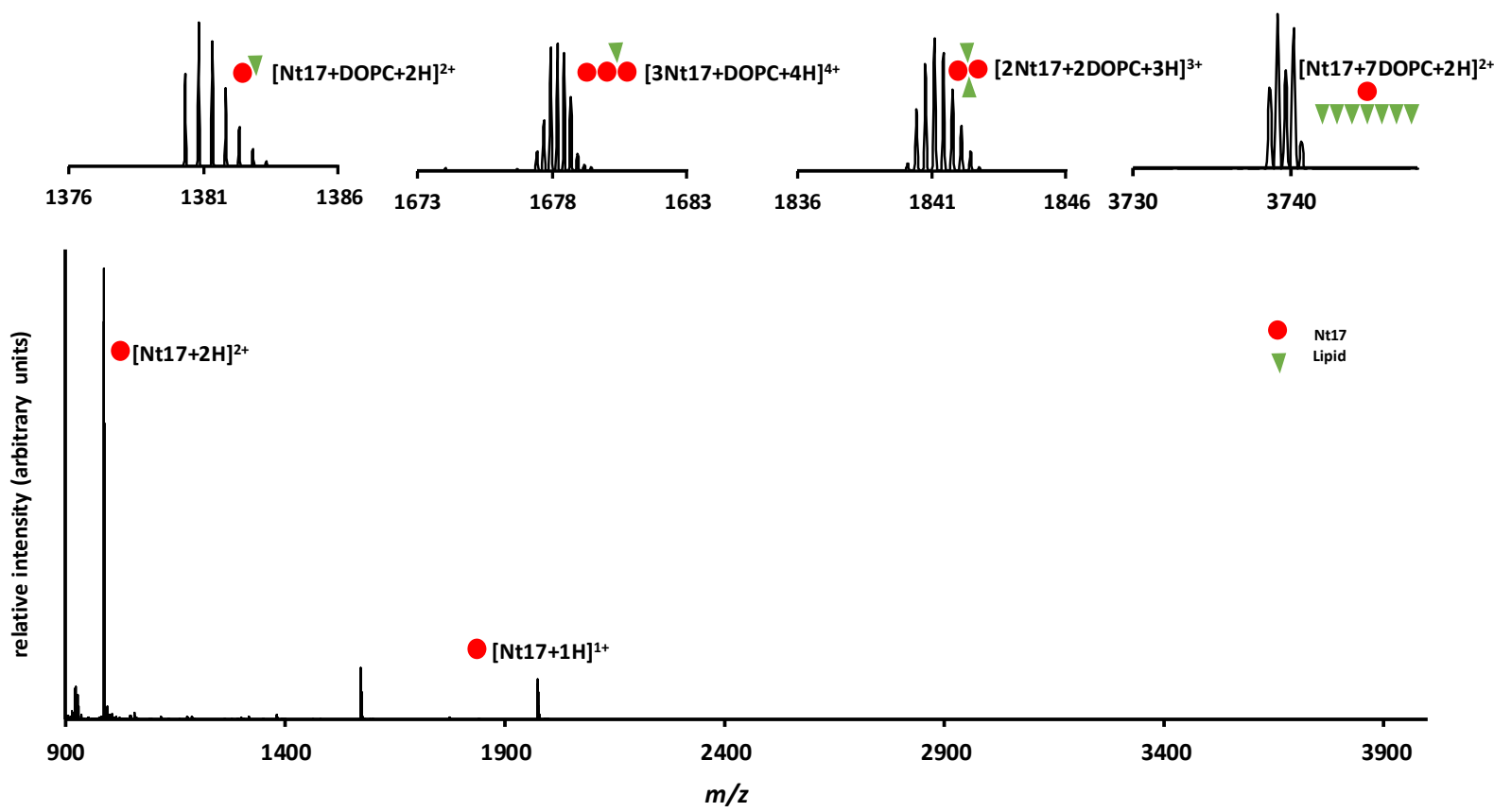

Figure 3. 1 Full range mass spectrum of the peptide-DOPC system.

For labeling purposes, a large red circle represents a single Nt17 peptide, a green triangle represents a single DOPC lipid molecule. Insets show expanded regions for a number of peptide-lipid complex ions. Ion identities are shown as symbol representations. Reproduced with permission from the Journal of Mass Spectrometry: Investigating the interactions of the first 17 amino acid residues of Huntingtin with lipid vesicles using mass spectrometry and molecular dynamics. Kiani Karanji, A., Beasley, M., Sharif, D., Ranjbaran A., Legleiter, J. and Valentine, S. J. Journal of Mass Spectrometry 55 (1), e4470. Copyright @ 2019 John Wiley \& Sons, Ltd. 
In addition to the ions described above, ions comprised of peptide-lipid complexes are also observed. These range in size from peptide monomer to tetramer ions. A complete list of the observed protein-lipid complex ions is presented in Table 3.1. Notably, monomer peptide ions with differing numbers of lipid adducts are observed. These range in size from 1 to 7 attached lipids. This relatively high number of adducts could, to some degree, indicate the propensity of the lipid to interact with this peptide (see discussion below). In addition to these ions, peptide-lipid complexes which include one lipid attached to dimer and trimer species are observed at $\mathrm{m} / \mathrm{z} \sim 1578$ and 1678 , respectively, Figure 3.1). Table 3.1 shows the multimeric peptide-lipid ions that have been observed in these studies. In general, the number of separate ions as well as the number of attached lipids decreases with higher order peptide multimer. For example, $>1$ lipid adduct is observed for all the peptide multimers except for the tetramer where only 1 lipid is attached (Table 3.1). 


\begin{tabular}{|c|c|c|c|c|c|c|c|c|}
\hline $\operatorname{lon}^{\mathrm{a}}$ & $m / z^{b}$ & $\begin{array}{c}\text { Charge } \\
\text { state }\end{array}$ & $\operatorname{lon}^{\mathrm{a}}$ & $m / z^{b}$ & $\begin{array}{c}\text { Charge } \\
\text { state }\end{array}$ & $\operatorname{Ion}^{\mathrm{a}}$ & $m / z^{b}$ & $\begin{array}{c}\text { Charge } \\
\text { state }\end{array}$ \\
\hline Nt17 + 1DOPC & 2760.64 & 1 & Nt17 + 1POPC & 2734.63 & 1 & Nt17 + 1POPE & 2692.59 & 1 \\
\hline Nt17 + 1DOPC & 1380.82 & 2 & Nt17 + 1POPC & 1367.81 & 2 & Nt17 + 1POPE & 1346.79 & 2 \\
\hline $1 \mathrm{Nt} 17+2 \mathrm{DOPC}$ & 3546.24 & 1 & $\mathrm{Nt17}+2 \mathrm{POPC}$ & 1747.61 & 2 & Nt17 + 2POPE & 1705.57 & 2 \\
\hline $\mathrm{Nt17}+2 \mathrm{DOPC}$ & 1773.62 & 2 & $\mathrm{Nt17}+3 \mathrm{POPC}$ & 2127.40 & 2 & Nt17 + 3POPE & 2064.34 & 2 \\
\hline Nt17 + 3DOPC & 2166.42 & 2 & $\mathrm{Nt17}+4 \mathrm{POPC}$ & 2507.2 & 2 & $\mathrm{Nt} 17+4 \mathrm{POPE}$ & 2423.12 & 2 \\
\hline $\mathrm{Nt} 17+4 \mathrm{DOPC}$ & 2559.22 & 2 & $\mathrm{Nt} 17+5 \mathrm{POPC}$ & 2886.99 & 2 & $\mathrm{Nt} 17+5 \mathrm{POPE}$ & 2781.89 & 2 \\
\hline $\mathrm{Nt17}+5 \mathrm{DOPC}$ & 2952.02 & 2 & $\mathrm{Nt} 17+6 \mathrm{POPC}$ & 3266.79 & 2 & $2 \mathrm{Nt} 17+1 \mathrm{POPE}$ & 2333.81 & 2 \\
\hline $\mathrm{Nt} 17+6 \mathrm{DOPC}$ & 3344.82 & 2 & $2 \mathrm{Nt} 17+1 \mathrm{POPC}$ & 2354.83 & 2 & $2 \mathrm{Nt} 17+1 \mathrm{POPE}$ & 1556.21 & 3 \\
\hline $\mathrm{Nt} 17+7 \mathrm{DOPC}$ & 3737.62 & 2 & $2 \mathrm{Nt} 17+1 \mathrm{POPC}$ & 1570.22 & 3 & $2 \mathrm{Nt} 17+2 \mathrm{POPE}$ & 1795.39 & 3 \\
\hline $2 \mathrm{Nt} 17+1 \mathrm{DOPC}$ & 2367.84 & 2 & $2 \mathrm{Nt} 17+2 \mathrm{POPC}$ & 1823.42 & 3 & $2 \mathrm{Nt} 17+3 \mathrm{POPE}$ & 2034.57 & 3 \\
\hline $2 \mathrm{Nt} 17+1 \mathrm{DOPC}$ & 1578.89 & 3 & $2 \mathrm{Nt} 17+3 \mathrm{POPC}$ & 2076.61 & 3 & & & \\
\hline $2 \mathrm{Nt} 17+2 \mathrm{DOPC}$ & 2760.84 & 2 & $2 \mathrm{Nt} 17+4 \mathrm{POPC}$ & 2329.81 & 3 & & & \\
\hline $2 \mathrm{Nt} 17+2 \mathrm{DOPC}$ & 1840.76 & 3 & $2 \mathrm{Nt} 17+5 \mathrm{POPC}$ & 2583.01 & 3 & & & \\
\hline $2 \mathrm{Nt} 17+3 \mathrm{DOPC}$ & 3153.44 & 2 & $3 \mathrm{Nt} 17+1 \mathrm{POPC}$ & 2228.23 & 3 & & & \\
\hline $2 \mathrm{Nt} 17+3 \mathrm{DOPC}$ & 2102.62 & 3 & $3 \mathrm{Nt} 17+2 \mathrm{POPC}$ & 2481.43 & 3 & & & \\
\hline $2 \mathrm{Nt} 17+4 \mathrm{DOPC}$ & 3546.24 & 2 & $3 \mathrm{Nt} 17+1 \mathrm{POPC}$ & 1671.42 & 4 & & & \\
\hline $2 \mathrm{Nt} 17+4 \mathrm{DOPC}$ & 2364.49 & 3 & $3 \mathrm{Nt} 17+2 \mathrm{POPC}$ & 1861.32 & 4 & & & \\
\hline $2 \mathrm{Nt} 17+5 \mathrm{DOPC}$ & 3939.04 & 2 & $4 \mathrm{Nt} 17+1 \mathrm{POPC}$ & 2164.93 & 4 & & & \\
\hline $2 \mathrm{Nt} 17+5 \mathrm{DOPC}$ & 2626.36 & 3 & & & & & & \\
\hline $2 \mathrm{Nt} 17+5 \mathrm{DOPC}$ & 1970.02 & 4 & & & & & & \\
\hline $2 \mathrm{Nt} 17+6 \mathrm{DOPC}$ & 2888.22 & 3 & & & & & & \\
\hline $2 \mathrm{Nt} 17+7 \mathrm{DOPC}$ & 3150.09 & 3 & & & & & & \\
\hline $2 \mathrm{Nt} 17+8 \mathrm{DOPC}$ & 3411.96 & 3 & & & & & & \\
\hline $3 \mathrm{Nt} 17+1 \mathrm{DOPC}$ & 3354.86 & 2 & & & & & & \\
\hline $3 \mathrm{Nt} 17+1 \mathrm{DOPC}$ & 2236.90 & 3 & & & & & & \\
\hline $3 \mathrm{Nt} 17+1 \mathrm{DOPC}$ & 1677.93 & 4 & & & & & & \\
\hline $3 \mathrm{Nt} 17+2 \mathrm{DOPC}$ & 2498.77 & 3 & & & & & & \\
\hline $3 \mathrm{Nt} 17+2 \mathrm{DOPC}$ & 1874.33 & 4 & & & & & & \\
\hline $3 \mathrm{Nt} 17+3 \mathrm{DOPC}$ & 2070.73 & 4 & & & & & & \\
\hline $3 \mathrm{Nt} 17+4 \mathrm{DOPC}$ & 3022.50 & 3 & & & & & & \\
\hline $3 \mathrm{Nt} 17+4 \mathrm{DOPC}$ & 2267.13 & 4 & & & & & & \\
\hline $3 \mathrm{Nt} 17+5 \mathrm{DOPC}$ & 3284.37 & 3 & & & & & & \\
\hline $3 \mathrm{Nt} 17+5 \mathrm{DOPC}$ & 2463.53 & 4 & & & & & & \\
\hline $3 \mathrm{Nt} 17+6 \mathrm{DOPC}$ & 2659.93 & 4 & & & & & & \\
\hline $3 \mathrm{Nt} 17+7 \mathrm{DOPC}$ & 2856.33 & 4 & & & & & & \\
\hline $3 \mathrm{Nt} 17+8 \mathrm{DOPC}$ & 3052.73 & 4 & & & & & & \\
\hline $4 \mathrm{Nt} 17+1 \mathrm{DOPC}$ & 2894.92 & 3 & & & & & & \\
\hline $4 \mathrm{Nt} 17+1 \mathrm{DOPC}$ & 2171.44 & 4 & & & & & & \\
\hline
\end{tabular}

${ }^{\text {a }}$ The following average masses has been used to assign the peaks: 1974.04 for Nt17 peptide, 785.60 for DOPC lipid and 717.54 for POPE lipid.

Table 3. 1 Assigned compounds and their corresponding mass to charge ratio 
Lipid preference of Nt17. The affinity of amphipathic a-helices for lipid bilayers has been shown to be dependent on the physicochemical characteristics of the membrane ${ }^{65-67}$. One characteristic showing importance is the packing defects of the membrane. Beyer et al. have suggested that a small radius of curvature is essential for increasing peptide-membrane interaction ${ }^{68}$. In this study it was suggested that peptide binding to a membrane depends on the presence of defects in the membrane structure at the membrane-water interface. These shortlived defects create temporary spacing between the lipid head groups which allows greater interaction between the lipid and the peptide. To investigate this phenomenon further by MS, experiments were also conducted using DOPC, POPC, and POPE lipid systems. All are zwitterionic lipids; however, the DOPC lipid contains a much bulkier head group which is expected to increase the overall area per lipid ${ }^{69}$. Additionally, DOPC and POPE systems have different lipid tails, that can impact their fluidity at given temperatures. DOPC, POPC, and POPE have respective phase transition temperatures of $-17,-2$ and $25^{\circ} \mathrm{C}$. Thus the DOPC bilayer system is more fluid under experimental conditions $\left(\sim 37^{\circ} \mathrm{C}\right)$ which can more readily create short-lived defects in the exposed membrane leaflet.

By comparing the mass spectral results from the Nt17-DOPC (Figure 3.1), and Nt-17POPC and Nt17-POPE (Figure S1 in the Supporting Information section (Appendix B)) systems, it may be possible to obtain some information about differences in types of interaction between the peptide and the respective lipid vesicles. The assigned peaks for the peptide-PC and peptide-PE complex ions are listed in Table 1. From Table 3.1, it is readily apparent that the most lipid adducts are observed in the DOPC system. For example, $1 \mathrm{Nt17}$ peptide is observed with up to 7 attached DOPC lipids. Conversely, in the POPE system, only up to 5 lipid adducts are observed for the monomer peptide. In comparison, this number is 6 for the POPC system.

The greater propensity of the peptide to bind with DOPC extends to the higher-order oligomers of Nt17. For the DOPC system up to 8 lipid adducts are observed with peptide 
dimers while this limit is 5 for POPC and 3 for POPE systems. There was no mass spectral feature corresponding to the peptide trimer and tetramer containing POPE lipid adduct but the peak for tetramer with one lipid adduct can be seen at $m / z \sim 2171.44$ for the DOPC system. Overall, 38 lipid adduct species are observed for the DOPC vesicle system while only 10 related peaks are observed for the POPE vesicle system suggesting the possibility of a different interaction type for the former lipid vesicles. Notably, the phenomenon of increased lipid adducts in the DOPC system is not a result of the relative number of peptide monomers or multimers believed to form in solution as these are observed in similar abundances for all peptide-lipid systems. Together these results suggest that differences in interaction between the Nt17 peptide individual lipid vesicles may be responsible for differences in the mass spectra.

Co-mixing Nt17 peptide and lipids without vesicle formation. To investigate the role of the ESI process on the numbers of $\mathrm{Nt17}$ peptide ions with lipid adducts, experiments were performed in which the peptide and lipid were co-mixed and, after a similar incubation time, electrosprayed into the mass spectrometer. More specifically, the Nt17 peptide and lipids were mixed without any preparation (without sonication and freeze drying) for 5 hours in $10 \mathrm{mM}$ ammonium acetate buffer. The assigned mass spectral peaks for these datasets are shown in bold in Table 1. Compared to the experiments in which lipid vesicles were generated before adding the peptide, in all cases, co-mixing peptide and lipid showed a significantly decreased number of assigned species. Only 4 ions $\left([\mathrm{Nt} 17+1 \mathrm{DOPC}+\mathrm{H}]^{+}, \quad[\mathrm{Nt} 17+1 \mathrm{DOPC}+2 \mathrm{H}]^{2+}\right.$, $[\mathrm{Nt} 17+2 \mathrm{DOPC}+2 \mathrm{H}]^{2+}$, and $[\mathrm{Nt} 17+3 \mathrm{DOPC}+2 \mathrm{H}]^{2+}$ at $\mathrm{m} / \mathrm{z} 2760.64,1380.82,1773.62$, and 2166.42 relatively) were assigned for the Nt17-DOPC system Table 3.1. For the Nt17-POPC and for the Nt17-POPE systems, only 6 and 3 species were observed, respectively. This indicates that vesicle formation is an important step to detect elevated peptide-lipid interactions. It also supports the premise that the mass spectral peak diversity is an indication of solution interaction as opposed to only processes occurring in the ESI droplets. 
As a final consideration for co-mixing experiments, it is noted that only 2 of 13 assigned peaks are for Nt17 dimers with lipid adducts. This could suggest that Nt17 requires a membrane interface to form increased numbers of oligomeric species and this is revealed in the MS data. This would be consistent with the concept of mode of action for amphipathic helices as well as proposed mechanisms of oligomer formation for $h t^{33}$. That said, caution must be used in interpreting the mass spectral data as the dataset features could arise in another manner. For example, an increased degree of interaction with the lipid membrane could translate into a large population of adsorbed monomer. During the ESI process, this increased likelihood of being located at the membrane surface could result in an increase in peptide numbers within ESI progeny droplets resulting in multimeric ions. Therefore, no conclusions can be drawn with regard to the degree of membrane-induced oligomerization. To disentangle the routes of formation for such a heterogenous mixture of oligomeric species is the subject of future, more extensive experiments employing a greater number of lipid systems as well as gasphase separations and reactivity studies ${ }^{26,70-73}$. This process will also be examined by MD simulations where multiple peptides are examined with the membrane systems. This preliminary study does, however, show that vesicle formation is required to produce a greater number of oligomer ions with lipids attached in the mass spectrum.

Consideration of peptide-lipid ion intensities. It is instructive to consider the ion intensities with regard to the degree of interaction between peptides and lipid vesicles. Figure S2 in the Supporting Information section (Appendix B) shows the relative intensities of the various ions observed for the three separate lipid systems. Notably, the ions with PE adducts are of higher intensity especially for those species containing 1 lipid adduct. The question arises as to the origin of this higher intensity in light of the observation that a greater number of diverse lipidated species is observed for DOPC and POPC systems. In other words, is this value reflective of greater interaction overall (independent of type) at the membrane surface? Here, a 
note of caution is raised based on a few observations. First, separate experiments have been performed on a LTQ mass spectrometer in which the $[\mathrm{Nt} 17+\mathrm{L}+2 \mathrm{H}]^{2+}$ (where $\mathrm{L}$ is DOPC or POPE) ions were isolated in the linear ion trap. Immediately upon isolation, the DOPCcontaining ions are observed to dissociate to some degree to yield $[\mathrm{Nt} 17+\mathrm{H}]^{+}$ions. This did not occur for the POPE-containing ions as shown in Figure S3 in the Supporting Information section (Appendix B). Based on this observation, it is possible that many ions with differently-oriented lipid adducts are very fragile and do not survive to the point of mass detection. Additionally, this could extend to the oligomeric species where a greater number of unique ions is observed for DOPC (Table 3.1). Due to such differences, it may not be possible to be fully determine whether or not ion intensity affords a clear indication of the degree of surface interaction. The results are presented here as being of interest and worthy of further MS and molecular modeling studies.

Molecular dynamics (MD) simulations: random coil interaction. To consider initial interactions between the Nt17 peptide and the lipid membrane, MD simulations were performed in which the initial peptide structure was random coil (see Experimental Section). To ensure that the proper parameters had been utilized for the simulations, the area per lipid was determined as a function of MD trajectory time. These results are shown for the DOPC, POPC, and POPE lipid systems in Figure 3.2. The average area per lipid values for the respective lipid systems are $68.6,65.1,57.3 \AA^{2}$ (Figure $3.2 A$ and Supplementary Table 1 (Appendix B)) This is consistent with the ordering and magnitudes of the reported areas per lipid and transition temperatures for DOPC ${ }^{74}, \mathrm{POPC}^{75}$, and POPE ${ }^{69}$. 

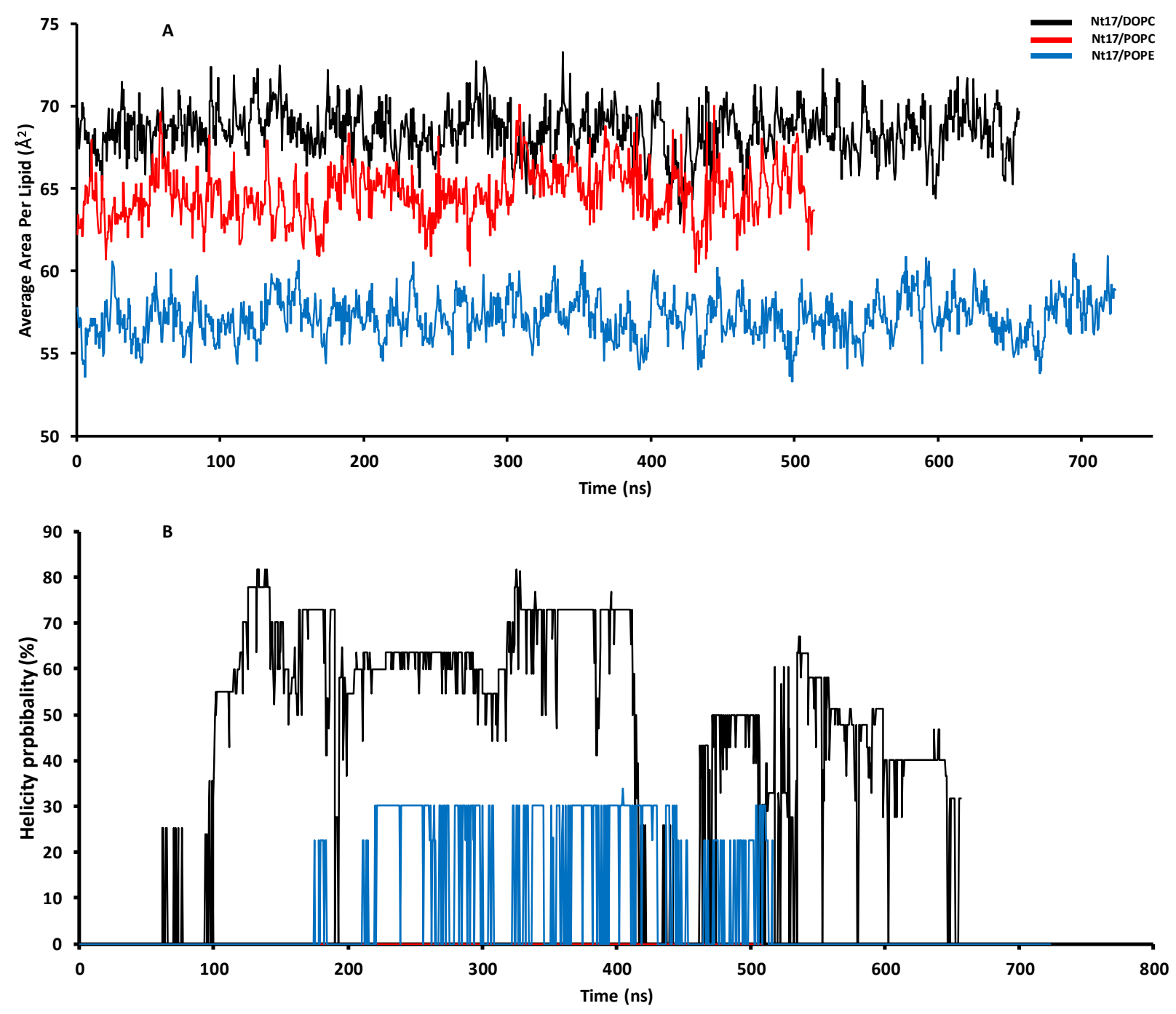

Figure 3. 2 Area per lipid calculations of the 100-member lipid bilayer for the DOPC, POPC and POPE and relative helical propensity for $\mathrm{Nt} 17$ peptide in the presence of these lipid vesicles.

A) Theoretical area per lipid calculations of the 100-member lipid bilayer for the DOPC, POPC and POPE. The linear relationship shows that the average area per lipid number for each DOPC, POPC, and POPE are 68.59, 65.12, 57.26 respectively. B) the relative degree of peptide helicity as a function of simulation time for all three lipid systems. Here the initial structure for the Nt17 peptide was completely random coil. Reproduced with permission from the Journal of Mass Spectrometry: Investigating the interactions of the first 17 amino acid residues of Huntingtin with lipid vesicles using mass spectrometry and molecular dynamics. Kiani Karanji, A., Beasley, M., Sharif, D., Ranjbaran A., Legleiter, J. and Valentine, S. J. Journal of Mass Spectrometry 55 (1), e4470. Copyright @ 2019 John Wiley \& Sons, Ltd. 
One measurement that provides some insight into interaction differences with the random coil peptide is the degree of helicity within the peptide as a function of MD trajectory time. Figure $3.2 \mathrm{~B}$ shows the results for the three different lipid systems. Of note is the increased degree of helicity achieved for the peptide in the DOPC system simulation. There are two major times in which extensive helix formation is observed occurring at $\sim 100$ and $\sim 470$ ns. It is instructive to observe how helix formation proceeds near these time points. Figure 3.3 shows this for the first time point region. In both instances, the $\mathrm{Nt} 17$ peptide is observed to interact with the membrane via the first 2 to 3 amino acid residues. Over the next few ns in the simulation, a single turn to two helical turns is observed. This rapidly propagates to extensive helical formation ( $50 \%$ in both cases) with concomitant detachment from the membrane surface. Notably, the degree of helix formation for the POPC and POPE lipid systems does not occur to the degree observed in the two instances for DOPC. 


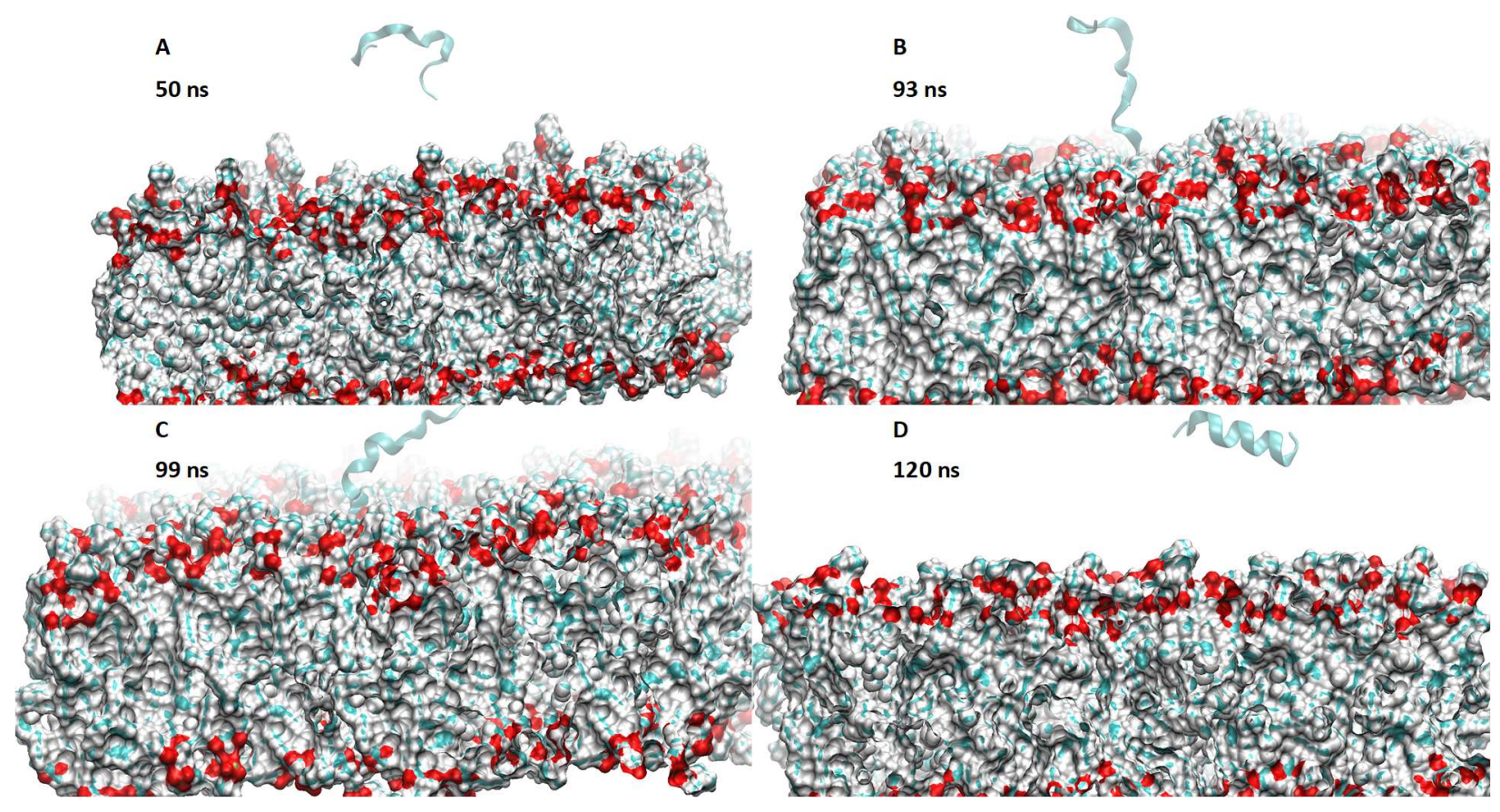

Figure 3. 3 Schematic representations of random coil Nt17 peptide interacting with the DOPC bilayer.

A) The Nt17 peptide in solution having no contact with the bilayer. B) The peptide interacting with the bilayer surface after 93 ns simulation time. C) DOPC bilayer inducing some peptide helical formation. D) The Nt17 peptide leaves the bilayer surface with $\sim 65 \%$ helicity at a time of 120 ns. The peptides and DOPC lipid bilayer are shown with the "new cartoon" and "surf" drawing models, respectively. Reproduced with permission from the Journal of Mass Spectrometry: Investigating the interactions of the first 17 amino acid residues of Huntingtin with lipid vesicles using mass spectrometry and molecular dynamics. Kiani Karanji, A., Beasley, M., Sharif, D., Ranjbaran A., Legleiter, J. and Valentine, S. J. Journal of Mass Spectrometry 55 (1), e4470. Copyright $\odot 2019$ John Wiley \& Sons, Ltd. 
Molecular dynamics (MD) simulations: helix-membrane compatibility. Although the simulations described above present an intriguing possibility regarding differences in helix initiation roles by different membrane types, it is possible that they do not fully represent the interactions expected for a system in which the peptide has been incubated extensively in the presence of the lipid vesicles. Because experimental evidence suggests that, at the membrane surface, the peptide is helical, MD simulations have also been performed in which the fully helical peptide is placed at the membrane surface. This was performed as the computational time required to observe full helix formation while at the membrane surface may be prohibitively long according to the random coil simulations.

For the helix-membrane studies, the peptide was placed equidistant (29 $\AA$ - center of the bilayer to peptide center of mass) above the three different lipid bilayer head groups. Figure 3.4A shows a heat map representing the distance between each residue in the peptide and the center of the lipid bilayer across the simulation time. For the peptide-DOPC system, the MD simulations suggest that the $\mathrm{N}$-terminus section of the peptide helps to maintain the entire peptide at the surface of the bilayer. This occurs during the first 50 nanoseconds of the simulation. The process initiates with the $\mathrm{N}$-terminal residues over this relatively short timescale. Specifically, the first three amino acid residues (M1, A2, and T3), are the first to locate closer to the lipid membrane (Figure 3.4A). 

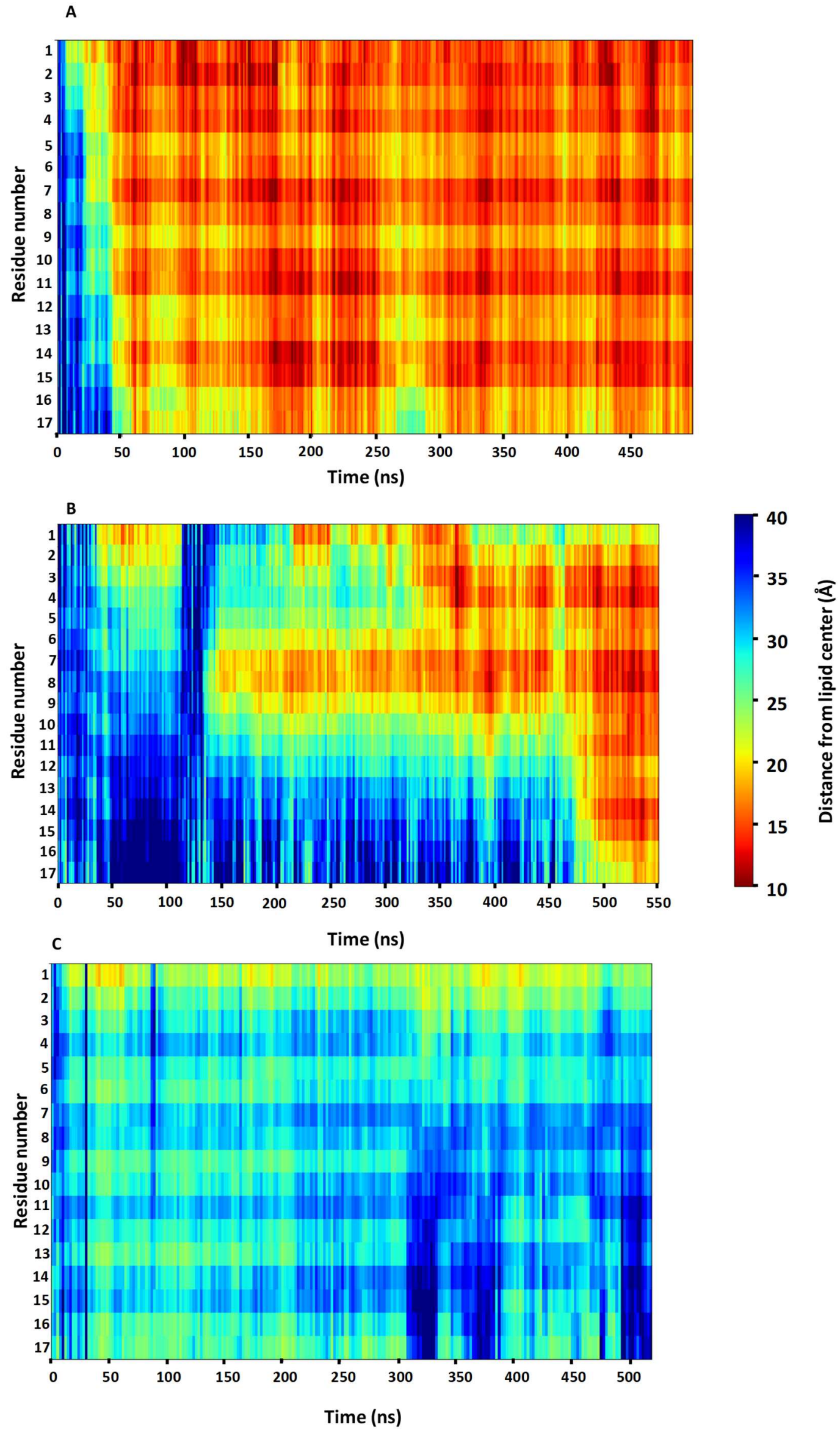
Figure 3. 4 Heat maps showing the distance between $\alpha$-carbons of each residue on the Nt17 peptide to the center of the bilayer as a function of MD simulation time.

Panels A, B and B show the results for the DOPC, POPC and the POPE bilayer systems, respectively. The legend in the figure shows the distance $(\AA)$ represented by each color. Reproduced with permission from the Journal of Mass Spectrometry: Investigating the interactions of the first 17 amino acid residues of Huntingtin with lipid vesicles using mass spectrometry and molecular dynamics. Kiani Karanji, A., Beasley, M., Sharif, D., Ranjbaran A., Legleiter, J. and Valentine, S. J. Journal of Mass Spectrometry 55 (1), e4470. Copyright (C 2019 John Wiley \& Sons, Ltd.

This is followed by positioning successive residues (moving toward the C-terminus) within increased proximity of the lipid membrane. Notably, the first three residues are not associated with the helical character of the peptide obtained from the protein databank. Figure 3.4A also provides information regarding the relative orientation of the peptide with respect to the DOPC lipid membrane. After 50 nanoseconds, distinct bands are observed in the heat map. This reveals increased proximity for specific regions of the peptide. For example, residues 1 through 4, 7 and 8, 10 and 11, and 14 and 15 are in much closer proximity to the lipid membrane. This result is consistent with the suggested structure and topology of the Nt17 peptide interacting with a lipid membrane from solid-state NMR (ssNMR) experiments ${ }^{33}$.

The MD simulations for the POPC lipid system (Figure 3.4B) show different behavior compared with the DOPC results. One similarity is that initial interaction at the N-terminus is observed for residues 1 and 2 after 50 ns. This, however, is shorter lived for the POPC system as it disappears after 50 ns. By 150 ns this initial interaction is replaced by primary interaction at residues 7 and 8 which persists for the remainder of the simulation. In a sense, this interaction anchors, to some degree, the peptide and allows other interactions to eventually occur. For example, the interaction for residue 1 and 2 is re-established at $\sim 220$ ns. Subsequent interactions for residues 3 and 4, 11, and 14 and 15 are established by $\sim 330,460$, 
and $490 \mathrm{~ns}$, respectively. The end result is the similar observance of bands associated with hydrophobic residues showing increased interaction; however, this association occurs at a much longer time point than that for the DOPC system.

The MD simulations for the POPE lipid membrane reveal a markedly different behavior regarding peptide-lipid interaction as shown in Figure 3.4C. As noted by the heat map, very little of the peptide is ever located within $20 \AA$ of the lipid membrane. As with the DOPC model, the first three residues are located closest to the lipid membrane across the simulation time frame. Indeed, periodically, the M1 residue is the only residue that locates within $20 \AA$ of the lipid membrane. Similar to the DOPC system, specific bands in the heat map (Figure 3.4C) reveal peptide residues exhibiting relatively increased proximity to the POPE lipid membrane; residues 1 and 2, 5 and 6,9 and 10, 12 and 13, and 16 and 17 are clearly located in increased proximity to the membrane across the simulation timescale. The clear differences between the modeled membrane systems are: 1) the residue numbers that are in the closest proximity; and, 2) the relative degree of proximity (observed to be much greater for the DOPC modeling).

Molecular Dynamics (MD) Simulations: peptide orientation. It is instructive to consider the origin of the bands presented in the heat maps in Figure 3.4. Notably, for all systems, the positioning of residues in closer proximity to the lipid membrane is $\sim 3$ to 4 residues apart. This distance in peptide primary sequence suggests a connection to helical structure. Additionally, the fact that different residues interact with the different lipid membranes suggests membrane-guided lipid orienting.

Because the Nt17 region is known to form an amphipathic helix ${ }^{76-77}$, it is instructive to examine the characteristics of the amino acid residues exhibiting greater lipid interaction. Figure 3.5A represents the nature of the peptide-lipid interaction for the DOPC system. Notably, because the same residues are involved for the POPC system (Figure 3.4), only the DOPC system is discussed here. As indicated by the heat map (Figure 3.4A), the peptide is noticeably 
close to the center of the bilayer. This can be observed pictorially in Figure 3.5A where the Nt17 peptide is located exactly at the membrane surface. At this moment in the simulation, the A2, L4, L7, F11 and L14 residues exhibit the greatest interaction with DOPC lipid molecules which matches with results from the previously performed ssNMR study ${ }^{33}$. Indeed, the general interaction is between these hydrophobic residues and the lipid tails (Figure $3.5 \mathrm{C}$ ). These interactions necessarily cause the more hydrophilic residues of the amphipathic helix to extend into the aqueous environment. This result is in very good agreement with previous simulations using this lipid system ${ }^{34}$.

Figure $3.5 \mathrm{~B}$ shows that the Nt17 peptide interacts more distantly with the POPE membrane surface. Figure $3.5 \mathrm{C}$ presents the interaction type between the peptide and one POPE lipid. This shows a simulation frame in which the peptide is located near its closest overall proximity to the lipid bilayer. Notably, the protonated K9 and K15 residues are shown to interact with the zwitterionic head groups. Indeed, the residues that exhibit the closest proximity to the lipid membrane across the dynamics run (Figure $3.4 \mathrm{C}$ ) are the hydrophilic K6, K9, S13, and $\mathrm{S} 16$ residues. It is noted that the $\mathrm{S} 16$ residue resides next to the protonated $\mathrm{K} 15$ residue. Overall, the peptide-lipid interactions are dominated by hydrophilic residues for the POPE system. 


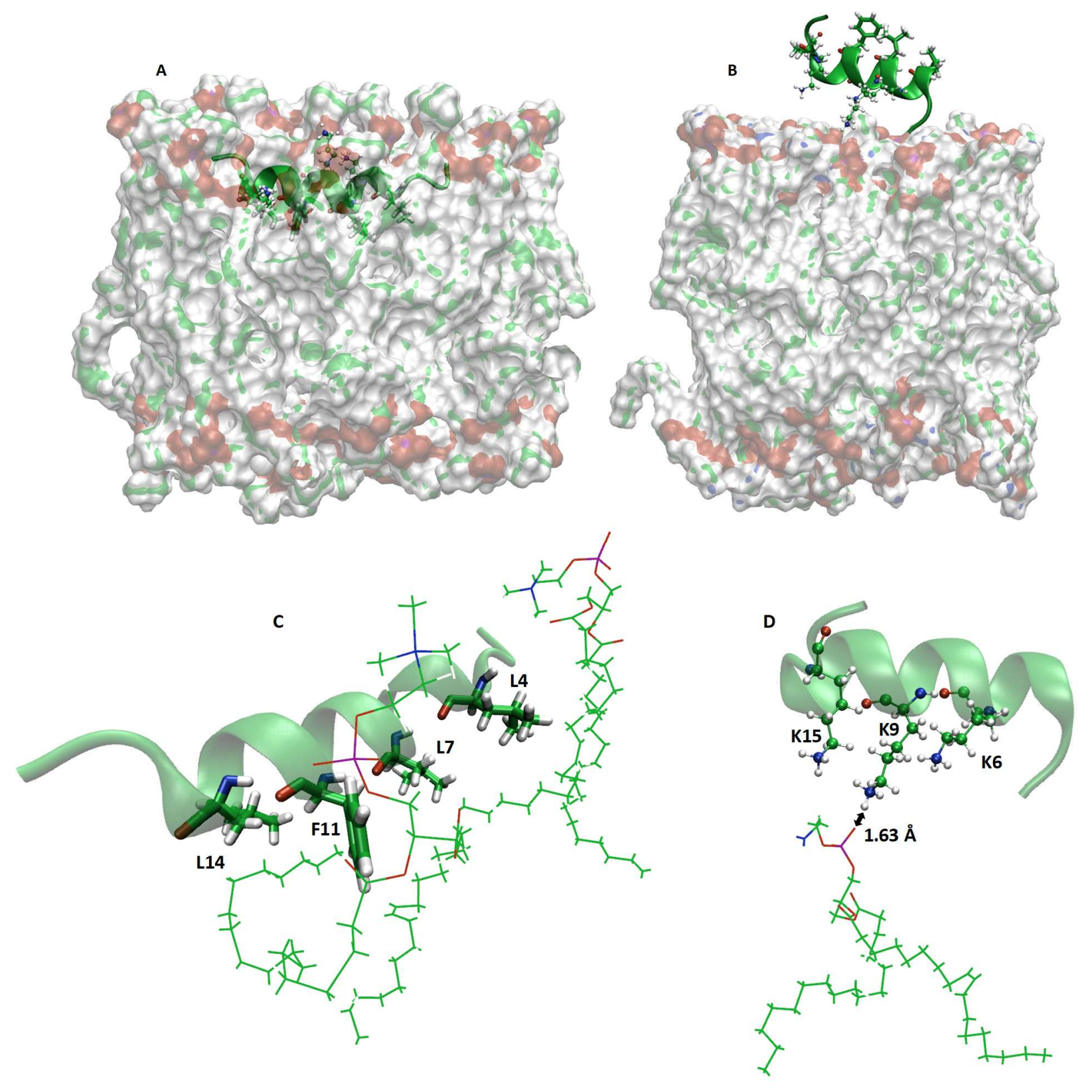

Figure 3. 5 Molecular representations of the position/orientation of the Nt17 peptide on the surface of the DOPC lipid bilayer, and the POPE lipid bilayer.

(A) the DOPC lipid bilayer, and (B) the POPE lipid bilayer. The peptides are shown with the ribbon rendering of the polypeptide backbone. Hydrophobic residues interacting with the DOPC lipids are rendered with the "licorice" drawing method and hydrophilic residues are represented by the "CPK" model. The lipid bilayer is depicted as an electron cloud rendering and the lipid head groups are emphasized. Panels $A$ and $B$ show results for the MD simulations for the DOPC and POPE lipid systems, respectively. Note that the DOPC system requires visualization 
from a top-down perspective because a side-on visualization does not reveal the peptide which is essentially interspersed within the lipid head groups. Schematic representations of the Nt17 peptide interacting with lipid molecules of the DOPC and POPE lipid systems are provided in panels $C$ and $D$, respectively. The peptides are shown with the ribbon rendering of the polypeptide backbone. Hydrophobic residues interacting with the DOPC lipids are rendered with the "licorice" drawing method and hydrophilic residues are represented by the "CPK" model. Interacting residues are labeled. A distance measurement is used to represent a hydrogen bond in the POPE system. Reproduced with permission from the Journal of Mass Spectrometry: Investigating the interactions of the first 17 amino acid residues of Huntingtin with lipid vesicles using mass spectrometry and molecular dynamics. Kiani Karanji, A., Beasley, M., Sharif, D., Ranjbaran A., Legleiter, J. and Valentine, S. J. Journal of Mass Spectrometry 55 (1), e4470. Copyright (C) 2019 John Wiley \& Sons, Ltd.

A question that arises is what is the net effect of the interactions described above on overall peptide orientation at the lipid membrane? Figure 3.5 helps to illustrate the net effect of these interactions. Overall, for the DOPC lipid system, the insertion of the hydrophobic residues to some degree anchors the peptide right at the lipid surface (i.e., among the head groups) as shown in Figure 3.5A. Therefore, there is greater disruption of the lipid bilayer at this location. Conversely, for the POPE system, the peptide is maintained at a distance from the lipid bilayer surface due to hydrogen bond interactions between charged residues and the head group constituents (Figure 3.5B). Therefore, the anchoring of the peptide occurs at several $\AA$ distance from the bilayer surface and the membrane integrity is largely preserved. It is important to note that the relative peptide orientation derived from the MD simulations (Figures 3.4 and 3.5) strongly supports the mass spectral data (Figure 3.1 and Table 3.1 ) discussed above. That is, the increased interaction with a greater number of lipid molecules for the DOPC system would suggest the observance of a greater number of lipid adducts ${ }^{36}$ as is observed in the mass spectra. Furthermore, the greater difficulty to establish the strongly adsorbed peptide for the 
POPC system (Figure 3.4B) could indicate the intermediate number of lipid adducts associated with this peptide-lipid system.

MD simulations: potential origin of interaction differences. Because the DOPC and the POPE lipids both contain zwitterionic head groups, it is instructive to consider differences that could account for the different predominant interactions observed with the MD simulations. One possibility is that the higher order of the POPE lipid bilayer disallows the interactions by the hydrophobic residues as are observed for the DOPC system. Here it is proposed that the POPE lipids can make strong intermolecular $\mathrm{H}$-bonds between the phosphate group on one lipid and the carbonyl oxygen atom of an adjacent lipid resulting in a higher ordering of the POPE bilayer. In contrast, the terminal methyl groups of the DOPC system disrupt such hydrogen bonding networks ${ }^{78}$ and, therefore, the head group spacing increases. As mentioned above, studies have hypothesized that the interaction between amphipathic $\alpha$-helices and membranes are influenced by short-lived defects created by membrane dynamics ${ }^{67-68,79}$. Thus, when a membrane is more fluid there will be more defects, and the peptide will have more opportunity to explore those defects leading to increased interactions with the lipid tails. Additionally, greater spacing between the headgroups resulting from membrane curvature may also lead to a higher propensity of peptide binding. ${ }^{30}$. Based on these hypotheses, it is instructive to compare the results with the average area per lipid calculations (Figure 3.2A). This analysis shows increasing numbers of lipidated species with increasing area per lipid. This supports the idea that the higher area per lipid value for the DOPC bilayer system could also provide more short-lived defects for the peptide interaction. The greater disorder (spacing) as well as the more fluid characteristics of the DOPC system would thus allow for increased interaction between the lipid tails and the hydrophobic residues as observed in Figure 3.5C.

MD simulations: peptide helical persistence. As mentioned above, the formation of an amphipathic $\alpha$-helix is hallmark of the $\mathrm{Nt17}$ tract ${ }^{80-84}$ in htt. Notably, it has previously been 
proposed that lipid interactions with the Nt17 amphipathic helix could occur precisely as suggested by the DOPC MD simulations. It has also been proposed that helix-helix interactions are required to form early-stage oligomers that ultimately lead to protein aggregation associated with $\mathrm{HD}^{81}$. It has also been suggested that peptide-lipid interaction could ultimately induce helix formation ${ }^{85}$. Therefore, it could be argued that suitable peptide-lipid interactions could favor the persistence of the amphipathic helix. The time-dependent helical character of the modeled Nt17 peptide has been assessed for the DOPC and POPE lipid systems investigated here. Figure S4 in the Supporting Information section (Appendix B) shows the helical content of the Nt17 peptide across the two different simulations. For the DOPC simulations, the peptide begins as relatively helical $(\sim 65 \%)$. The helicity is almost immediately disrupted within the first few nanoseconds and is reestablished at the $68 \%$ level for the first 70 ns before stabilizing at $\sim 75 \%$ for the remainder of the simulation. The initial collapse and the lower helicity correlate with the initial interaction of the $\mathrm{N}$-terminal region with the lipid bilayer (Figure 3.3A). The relatively stable region (sustained helicity) corresponds with increased interaction with the lipid. In comparison, for the POPE system, no initial collapse is evident, however, a greater number of collapse events is observed across the entire simulation. Additionally, the overall average level of helicity trends downward across the simulation and is ultimately observed to be well below that for the DOPC simulation. These observations are the same for the replicate analysis.

\section{Conclusions}

Electrospray ionization - mass spectrometry (ESI-MS) and molecular dynamics (MD) simulations have been used to study the lipid interaction properties of the first 17 amino acid residues of Huntingtin protein (htt). In this study, phosphatidylcholine (PC), the most common lipid in the eukaryotic membrane, as well as phosphatidylethanolamine (PE) lipids were used. 
From mass spectra data, a noticeable difference was observed in the number and type of peptide-lipid complex ions. The MS spectra indicate that the Nt17 peptide yields more adducts with the DOPC lipid in both monomeric and multimeric ion forms in comparison to the POPC and POPE lipids. MD simulations provide atomic insight into these interactions. The simulation showed that the residues of the Nt17 peptide were generally closer to the center of the DOPC bilayer throughout the simulation. The amphipathic peptide interacts with the DOPC bilayer with hydrophobic residues. However, when the POPE lipid bilayer was examined, the hydrophilic residues interact with the lipid headgroups. Further analysis utilizing the simulation data reveals that the area per lipid may play an important role in the Nt17-lipid interactions. The increased surface area for the DOPC bilayer results in a longer lasting interaction as the Nt17 peptide is imbedded within the lipid headgroups resulting in more effective interaction with the hydrophobic tails. The simulation also suggests that the increased interaction between the Nt17 peptide and the DOPC lipid bilayer leads to greater stabilization of helicity for the peptide. Conversely, as the peptide increases aqueous interactions as opposed to the lipid bilayer, it loses some helical character over time. As the Nt17 peptide is a lipid-binding domain, its interactions with the membrane can lead to the formation of helix bundles at the bilayer surface. Understanding the nature of these interactions is vital because they can trigger the aggregation process and ultimately result in disease.

\section{Acknowledgments}

We are grateful for financial support from the National Institutes of Health (R01GM114494). We are also grateful for stimulating conversations regarding molecular dynamics simulations with Professor Blake Mertz of West Virginia University. 


\section{References}

1. Persaud, R.; Boggs, J. M.; Wood, D. D.; Moscarello, M. A., Interaction of glycosylated human myelin basic protein with lipid bilayers. Biochemistry 1989, 28 (10), 4209-16.

2. Poklar, N.; Fritz, J.; Macek, P.; Vesnaver, G.; Chalikian, T. V., Interaction of the pore-forming protein equinatoxin II with model lipid membranes: A calorimetric and spectroscopic study. Biochemistry 1999, 38 (45), 14999-5008.

3. Shimizu, M.; Saito, M., Lipid-Protein Interaction at an Emulsified Oil Surface: Protein Structures and Their Roles in Lipid Binding. 1996, 650, 156-165.

4. Sanders, M. R.; Clifton, L. A.; Frazier, R. A.; Green, R. J., Role of Lipid Composition on the Interaction between a Tryptophan-Rich Protein and Model Bacterial Membranes. Langmuir 2016, 32 (8), 2050-7.

5. $\quad$ Nagy, L.; Milano, F.; Dorogi, M.; Agostiano, A.; Laczko, G.; Szebenyi, K.; Varo, G.; Trotta, M.; Maroti, P., Protein/lipid interaction in the bacterial photosynthetic reaction center: phosphatidylcholine and phosphatidylglycerol modify the free energy levels of the quinones. Biochemistry 2004, 43 (40), 12913-23.

6. Guigas, G.; Weiss, M., Effects of protein crowding on membrane systems. Biochim Biophys Acta 2016, 1858 (10), 2441-2450.

7. Han, S. K.; Kim, K. P.; Koduri, R.; Bittova, L.; Munoz, N. M.; Leff, A. R.; Wilton, D. C.; Gelb, M. H.; Cho, W. H., Roles of Trp(31) in high membrane binding and proinflammatory activity of human group $\mathrm{V}$ phospholipase A(2). Journal of Biological Chemistry 1999, 274 (17), 11881-11888.

8. Yang, J.; Bahreman, A.; Daudey, G.; Bussmann, J.; Olsthoorn, R. C.; Kros, A., Drug Delivery via Cell Membrane Fusion Using Lipopeptide Modified Liposomes. ACS Cent Sci 2016, 2 (9), 621-630.

9. Yin, H.; Flynn, A. D., Drugging Membrane Protein Interactions. Annu Rev Biomed Eng 2016, 18, 51-76.

10. Williams, A. F.; Barclay, A. N., The immunoglobulin superfamily--domains for cell surface recognition. Annu Rev Immunol 1988, 6, 381-405.

11. Simons, K.; Toomre, D., Lipid rafts and signal transduction. Nat Rev Mol Cell Biol 2000, 1 (1), 31-

9.

12. Resh, M. D., Membrane targeting of lipid modified signal transduction proteins. Subcell Biochem 2004, 37, 217-32.

13. Gorbenko, G. P.; Kinnunen, P. K., The role of lipid-protein interactions in amyloid-type protein fibril formation. Chem Phys Lipids 2006, 141 (1-2), 72-82.

14. Pandey, N. K.; Isas, J. M.; Rawat, A.; Lee, R. V.; Langen, J.; Pandey, P.; Langen, R., The 17-residuelong $\mathrm{N}$ terminus in huntingtin controls stepwise aggregation in solution and on membranes via different mechanisms. J Biol Chem 2018, 293 (7), 2597-2605.

15. Sparr, E.; Engel, M. F.; Sakharov, D. V.; Sprong, M.; Jacobs, J.; de Kruijff, B.; Hoppener, J. W.; Killian, J. A., Islet amyloid polypeptide-induced membrane leakage involves uptake of lipids by forming amyloid fibers. FEBS Lett 2004, 577 (1-2), 117-20.

16. Zhao, H.; Tuominen, E. K.; Kinnunen, P. K., Formation of amyloid fibers triggered by phosphatidylserine-containing membranes. Biochemistry 2004, 43 (32), 10302-7.

17. Avci, F. G.; Akbulut, B. S.; Ozkirimli, E., Membrane Active Peptides and Their Biophysical Characterization. Biomolecules 2018, 8 (3).

18. Sani, M. A.; Separovic, F., How Membrane-Active Peptides Get into Lipid Membranes. Acc Chem Res 2016, 49 (6), 1130-8. 
19. Gimenez-Andres, M.; Copic, A.; Antonny, B., The Many Faces of Amphipathic Helices. Biomolecules 2018, 8 (3).

20. Zhuchenko, O.; Bailey, J.; Bonnen, P.; Ashizawa, T.; Stockton, D. W.; Amos, C.; Dobyns, W. B.; Subramony, S. H.; Zoghbi, H. Y.; Lee, C. C., Autosomal dominant cerebellar ataxia (SCA6) associated with small polyglutamine expansions in the alpha 1A-voltage-dependent calcium channel. Nat Genet 1997, 15 (1), 62-9.

21. Rockabrand, E.; Slepko, N.; Pantalone, A.; Nukala, V. N.; Kazantsev, A.; Marsh, J. L.; Sullivan, P. G.; Steffan, J. S.; Sensi, S. L.; Thompson, L. M., The first 17 amino acids of Huntingtin modulate its subcellular localization, aggregation and effects on calcium homeostasis. Hum Mol Genet 2007, 16 (1), 6177.

22. Nozaki, K.; Onodera, O.; Takano, H.; Tsuji, S., Amino acid sequences flanking polyglutamine stretches influence their potential for aggregate formation. Neuroreport 2001, 12 (15), 3357-3364.

23. Wang, L.; Tsai, C. C., Atrophin proteins: an overview of a new class of nuclear receptor corepressors. Nucl Recept Signal 2008, 6, e009.

24. Panov, A. V.; Gutekunst, C. A.; Leavitt, B. R.; Hayden, M. R.; Burke, J. R.; Strittmatter, W. J.; Greenamyre, J. T., Early mitochondrial calcium defects in Huntington's disease are a direct effect of polyglutamines. Nat Neurosci 2002, 5 (8), 731-6.

25. Suopanki, J.; Gotz, C.; Lutsch, G.; Schiller, J.; Harjes, P.; Herrmann, A.; Wanker, E. E., Interaction of huntingtin fragments with brain membranes--clues to early dysfunction in Huntington's disease. $J$ Neurochem 2006, 96 (3), 870-84.

26. Arndt, J. R.; Kondalaji, S. G.; Maurer, M. M.; Parker, A.; Legleiter, J.; Valentine, S. J., Huntingtin NTerminal Monomeric and Multimeric Structures Destabilized by Covalent Modification of Heteroatomic Residues. Biochemistry 2015, 54 (28), 4285-96.

27. Cariulo, C.; Azzollini, L.; Verani, M.; Martufi, P.; Boggio, R.; Chiki, A.; Deguire, S. M.; Cherubini, M.; Gines, S.; Marsh, J. L.; Conforti, P.; Cattaneo, E.; Santimone, I.; Squitieri, F.; Lashuel, H. A.; Petricca, L.; Caricasole, A., Phosphorylation of huntingtin at residue T3 is decreased in Huntington's disease and modulates mutant huntingtin protein conformation. Proc Natl Acad Sci U S A 2017, 114 (50), E10809E10818.

28. Ehrnhoefer, D. E.; Sutton, L.; Hayden, M. R., Small changes, big impact: posttranslational modifications and function of huntingtin in Huntington disease. Neuroscientist 2011, 17 (5), 475-92.

29. Perutz, M. F.; Pope, B. J.; Owen, D.; Wanker, E. E.; Scherzinger, E., Aggregation of proteins with expanded glutamine and alanine repeats of the glutamine-rich and asparagine-rich domains of Sup35 and of the amyloid beta-peptide of amyloid plaques. Proc Natl Acad Sci U S A 2002, 99 (8), 5596-600.

30. Chaibva, M.; Burke, K. A.; Legleiter, J., Curvature enhances binding and aggregation of huntingtin at lipid membranes. Biochemistry 2014, 53 (14), 2355-65.

31. Chaibva, M.; Gao, X.; Jain, P.; Campbell, W. A. t.; Frey, S. L.; Legleiter, J., Sphingomyelin and GM1 Influence Huntingtin Binding to, Disruption of, and Aggregation on Lipid Membranes. ACS Omega 2018, $3(1), 273-285$.

32. Gao, X.; Campbell, W. A. t.; Chaibva, M.; Jain, P.; Leslie, A. E.; Frey, S. L.; Legleiter, J., Cholesterol Modifies Huntingtin Binding to, Disruption of, and Aggregation on Lipid Membranes. Biochemistry 2016, 55 (1), 92-102.

33. Michalek, M.; Salnikov, E. S.; Bechinger, B., Structure and topology of the huntingtin 1-17 membrane anchor by a combined solution and solid-state NMR approach. Biophys J 2013, 105 (3), 699710.

34. Cote, S.; Binette, V.; Salnikov, E. S.; Bechinger, B.; Mousseau, N., Probing the Huntingtin 1-17 membrane anchor on a phospholipid bilayer by using all-atom simulations. Biophys $J$ 2015, 108 (5), 1187-98. 
35. van den Heuvel, R. H.; Heck, A. J., Native protein mass spectrometry: from intact oligomers to functional machineries. Curr Opin Chem Biol 2004, 8 (5), 519-26.

36. Landreh, M.; Costeira-Paulo, J.; Gault, J.; Marklund, E. G.; Robinson, C. V., Effects of Detergent Micelles on Lipid Binding to Proteins in Electrospray lonization Mass Spectrometry. Anal Chem 2017, 89 (14), 7425-7430.

37. Walker, L. R.; Marzluff, E. M.; Townsend, J. A.; Resager, W. C.; Marty, M. T., Native Mass Spectrometry of Antimicrobial Peptides in Lipid Nanodiscs Elucidates Complex Assembly. Anal Chem 2019, 91 (14), 9284-9291.

38. Liu, Y.; Cong, X.; Liu, W.; Laganowsky, A., Characterization of Membrane Protein-Lipid Interactions by Mass Spectrometry Ion Mobility Mass Spectrometry. J Am Soc Mass Spectrom 2017, 28 (4), 579-586.

39. Marcoux, J.; Wang, S. C.; Politis, A.; Reading, E.; Ma, J.; Biggin, P. C.; Zhou, M.; Tao, H.; Zhang, Q.; Chang, G.; Morgner, N.; Robinson, C. V., Mass spectrometry reveals synergistic effects of nucleotides, lipids, and drugs binding to a multidrug resistance efflux pump. Proc Natl Acad Sci U S A 2013, 110 (24), 9704-9.

40. demmers, J. A.; Haverkamp, J.; Heck, A. J.; Koeppe, R. E., 2nd; Killian, J. A., Electrospray ionization mass spectrometry as a tool to analyze hydrogen deuterium exchange kinetics of transmembrane peptides in lipid bilayers. PNAS 2000, 97 (7), 3189-3194.

41. Patrick, J. W.; Gamez, R. C.; Russell, D. H., Elucidation of conformer preferences for a hydrophobic antimicrobial peptide by vesicle capture-freeze-drying: a preparatory method coupled to ion mobility-mass spectrometry. Anal Chem 2015, 87 (1), 578-83.

42. Scarff, C. A.; Ashcroft, A. E.; Radford, S. E., Characterization of Amyloid Oligomers by Electrospray Ionization-Ion Mobility Spectrometry-Mass Spectrometry (ESI-IMS-MS). In Protein Amyloid Aggregation: Methods and Protocols, Eliezer, D., Ed. Springer New York: New York, NY, 2016; pp 115132.

43. Ionut Iurascu, M.; Cozma, C.; Tomczyk, N.; Rontree, J.; Desor, M.; Drescher, M.; Przybylski, M., Structural characterization of beta-amyloid oligomer-aggregates by ion mobility mass spectrometry and electron spin resonance spectroscopy. Anal Bioanal Chem 2009, 395 (8), 2509-19.

44. Young, L. M.; Cao, P.; Raleigh, D. P.; Ashcroft, A. E.; Radford, S. E., Ion mobility spectrometrymass spectrometry defines the oligomeric intermediates in amylin amyloid formation and the mode of action of inhibitors. J Am Chem Soc 2014, 136 (2), 660-70.

45. Wang, J. S.; Whitehead, S. N.; Yeung, K. K., Detection of Amyloid Beta (Abeta) Oligomeric Composition Using Matrix-Assisted Laser Desorption Ionization Mass Spectrometry (MALDI MS). J Am Soc Mass Spectrom 2018, 29 (4), 786-795.

46. Bernstein, S. L.; Wyttenbach, T.; Baumketner, A.; Shea, J.-E.; Bitan, G.; Teplow, D. B.; Bowers, M. T., Amyloid $\beta$-Protein: Monomer Structure and Early Aggregation States of A $\beta 42$ and Its Pro19 Alloform. J Am Chem Soc 2005, 127 (7), 2075-2084.

47. Bernstein, S. L.; Dupuis, N. F.; Lazo, N. D.; Wyttenbach, T.; Condron, M. M.; Bitan, G.; Teplow, D. B.; Shea, J. E.; Ruotolo, B. T.; Robinson, C. V.; Bowers, M. T., Amyloid-beta protein oligomerization and the importance of tetramers and dodecamers in the aetiology of Alzheimer's disease. Nat Chem 2009, 1 (4), 326-31.

48. Do, T. D.; Economou, N. J.; Chamas, A.; Buratto, S. K.; Shea, J. E.; Bowers, M. T., Interactions between amyloid-beta and Tau fragments promote aberrant aggregates: implications for amyloid toxicity. J Phys Chem B 2014, 118 (38), 11220-30.

49. Pryor, N. E.; Moss, M. A.; Hestekin, C. N., Unraveling the early events of amyloid-beta protein (Abeta) aggregation: techniques for the determination of Abeta aggregate size. Int J Mol Sci 2012, 13 (3), 3038-72. 
50. Doherty, C. P. A.; Young, L. M.; Karamanos, T. K.; Smith, H. I.; Jackson, M. P.; Radford, S. E.; Brockwell, D. J., A peptide-display protein scaffold to facilitate single molecule force studies of aggregation-prone peptides. Protein Sci 2018, 27 (7), 1205-1217.

51. Osterlund, N.; Kulkarni, Y. S.; Misiaszek, A. D.; Wallin, C.; Kruger, D. M.; Liao, Q.; Mashayekhy Rad, F.; Jarvet, J.; Strodel, B.; Warmlander, S.; Ilag, L. L.; Kamerlin, S. C. L.; Graslund, A., Amyloid-beta Peptide Interactions with Amphiphilic Surfactants: Electrostatic and Hydrophobic Effects. ACS Chem Neurosci 2018, 9 (7), 1680-1692.

52. Osterlund, N.; Luo, J.; Warmlander, S.; Graslund, A., Membrane-mimetic systems for biophysical studies of the amyloid-beta peptide. Biochim Biophys Acta Proteins Proteom 2019, 1867 (5), 492-501.

53. Knight, P. D.; Karamanos, T. K.; Radford, S. E.; Ashcroft, A. E., Identification of a novel site of interaction between ataxin-3 and the amyloid aggregation inhibitor polyglutamine binding peptide 1. Eur J Mass Spectrom (Chichester) 2018, 24 (1), 129-140.

54. Chen, M.; Wolynes, P. G., Aggregation landscapes of Huntingtin exon 1 protein fragments and the critical repeat length for the onset of Huntington's disease. Proc Natl Acad Sci U S A 2017, 114 (17), 4406-4411.

55. Jayaraman, M.; Thakur, A. K.; Kar, K.; Kodali, R.; Wetzel, R., Assays for studying nucleated aggregation of polyglutamine proteins. Methods 2011, 53 (3), 246-54.

56. Sokolovski, M.; Sheynis, T.; Kolusheva, S.; Jelinek, R., Membrane interactions and lipid binding of casein oligomers and early aggregates. Biochim Biophys Acta 2008, 1778 (10), 2341-9.

57. Burke, K. A.; Kauffman, K. J.; Umbaugh, C. S.; Frey, S. L.; Legleiter, J., The interaction of polyglutamine peptides with lipid membranes is regulated by flanking sequences associated with huntingtin. J Biol Chem 2013, 288 (21), 14993-5005.

58. Hanwell, M. D.; Curtis, D. E.; Lonie, D. C.; Vandermeersch, T.; Zurek, E.; Hutchison, G. R., Avogadro: an advanced semantic chemical editor, visualization, and analysis platform. J Cheminform 2012, 4 (1), 17.

59. Jo, S.; Kim, T.; Iyer, V. G.; Im, W., CHARMM-GUI: a web-based graphical user interface for CHARMM. J Comput Chem 2008, 29 (11), 1859-65.

60. Phillips, J. C.; Braun, R.; Wang, W.; Gumbart, J.; Tajkhorshid, E.; Villa, E.; Chipot, C.; Skeel, R. D.; Kale, L.; Schulten, K., Scalable molecular dynamics with NAMD. J Comput Chem 2005, 26 (16), 1781-802.

61. Vanommeslaeghe, K.; Hatcher, E.; Acharya, C.; Kundu, S.; Zhong, S.; Shim, J.; Darian, E.; Guvench, O.; Lopes, P.; Vorobyov, I.; Mackerell, A. D., Jr., CHARMM general force field: A force field for drug-like molecules compatible with the CHARMM all-atom additive biological force fields. J Comput Chem 2010, 31 (4), 671-90.

62. Jo, S.; Kim, T.; Im, W., Automated builder and database of protein/membrane complexes for molecular dynamics simulations. PLoS One 2007, 2 (9), e880.

63. Allen, W. J.; Lemkul, J. A.; Bevan, D. R., GridMAT-MD: a grid-based membrane analysis tool for use with molecular dynamics. J Comput Chem 2009, 30 (12), 1952-8.

64. Kegel, K. B.; Sapp, E.; Yoder, J.; Cuiffo, B.; Sobin, L.; Kim, Y. J.; Qin, Z. H.; Hayden, M. R.; Aronin, N.; Scott, D. L.; Isenberg, G.; Goldmann, W. H.; DiFiglia, M., Huntingtin associates with acidic phospholipids at the plasma membrane. J Biol Chem 2005, 280 (43), 36464-73.

65. Drin, G.; Antonny, B., Amphipathic helices and membrane curvature. FEBS Lett 2010, 584 (9), 1840-7.

66. Hatzakis, N. S.; Bhatia, V. K.; Larsen, J.; Madsen, K. L.; Bolinger, P. Y.; Kunding, A. H.; Castillo, J.; Gether, U.; Hedegard, P.; Stamou, D., How curved membranes recruit amphipathic helices and protein anchoring motifs. Nat Chem Biol 2009, 5 (11), 835-41.

67. Cornell, R. B.; Taneva, S. G., Amphipathic helices as mediators of the membrane interaction of amphitropic proteins, and as modulators of bilayer physical properties. Current Protein \& Peptide Science 2006, 7 (6), 539-552. 
68. Nuscher, B.; Kamp, F.; Mehnert, T.; Odoy, S.; Haass, C.; Kahle, P. J.; Beyer, K., Alpha-synuclein has a high affinity for packing defects in a bilayer membrane: a thermodynamics study. $J$ Biol Chem 2004, 279 (21), 21966-75.

69. Jambeck, J. P.; Lyubartsev, A. P., An Extension and Further Validation of an All-Atomistic Force Field for Biological Membranes. J Chem Theory Comput 2012, 8 (8), 2938-48.

70. Khakinejad, M.; Ghassabi Kondalaji, S.; Tafreshian, A.; Valentine, S. J., Comprehensive Gas-Phase Peptide Ion Structure Studies Using Ion Mobility Techniques: Part 2. Gas-Phase Hydrogen/Deuterium Exchange for Ion Population Estimation. J Am Soc Mass Spectrom 2017, 28 (5), 960-970.

71. Ranganathan, N.; Li, C.; Suder, T.; Karanji, A. K.; Li, X.; He, Z.; Valentine, S. J.; Li, P., Capillary Vibrating Sharp-Edge Spray Ionization (cVSSI) for Voltage-Free Liquid Chromatography-Mass Spectrometry. J Am Soc Mass Spectrom 2019, 30 (5), 824-831.

72. Majuta, S. N.; Maleki, H.; Kiani Karanji, A.; Attanyake, K.; Loch, E.; Valentine, S. J., Magnifying ion mobility spectrometry-mass spectrometry measurements for biomolecular structure studies. Curr Opin Chem Biol 2018, 42, 101-110.

73. Ghassabi Kondalaji, S.; Khakinejad, M.; Tafreshian, A.; S, J. V., Comprehensive Peptide Ion Structure Studies Using Ion Mobility Techniques: Part 1. An Advanced Protocol for Molecular Dynamics Simulations and Collision Cross-Section Calculation. J Am Soc Mass Spectrom 2017, 28 (5), 947-959.

74. Kucerka, N.; Nagle, J. F.; Sachs, J. N.; Feller, S. E.; Pencer, J.; Jackson, A.; Katsaras, J., Lipid bilayer structure determined by the simultaneous analysis of neutron and X-ray scattering data. Biophys $J \mathbf{2 0 0 8}$, 95 (5), 2356-67.

75. Kucerka, N.; Nieh, M. P.; Katsaras, J., Fluid phase lipid areas and bilayer thicknesses of commonly used phosphatidylcholines as a function of temperature. Biochim Biophys Acta 2011, 1808 (11), 2761-71.

76. Adegbuyiro, A.; Sedighi, F.; Pilkington, A. W. t.; Groover, S.; Legleiter, J., Proteins Containing Expanded Polyglutamine Tracts and Neurodegenerative Disease. Biochemistry 2017, 56 (9), 1199-1217.

77. Burke, K. A.; Godbey, J.; Legleiter, J., Assessing mutant huntingtin fragment and polyglutamine aggregation by atomic force microscopy. Methods 2011, 53 (3), 275-84.

78. Murzyn, K.; Rog, T.; Pasenkiewicz-Gierula, M., Phosphatidylethanolamine-phosphatidylglycerol bilayer as a model of the inner bacterial membrane. Biophys $J$ 2005, 88 (2), 1091-103.

79. Drin, G.; Casella, J. F.; Gautier, R.; Boehmer, T.; Schwartz, T. U.; Antonny, B., A general amphipathic alpha-helical motif for sensing membrane curvature. Nat Struct Mol Biol 2007, 14 (2), 13846.

80. Bhattacharyya, A.; Thakur, A. K.; Chellgren, V. M.; Thiagarajan, G.; Williams, A. D.; Chellgren, B. W.; Creamer, T. P.; Wetzel, R., Oligoproline effects on polyglutamine conformation and aggregation. J Mol Biol 2006, 355 (3), 524-35.

81. Jayaraman, M.; Kodali, R.; Sahoo, B.; Thakur, A. K.; Mayasundari, A.; Mishra, R.; Peterson, C. B.; Wetzel, R., Slow amyloid nucleation via alpha-helix-rich oligomeric intermediates in short polyglutaminecontaining huntingtin fragments. J Mol Biol 2012, 415 (5), 881-99.

82. Jayaraman, M.; Mishra, R.; Kodali, R.; Thakur, A. K.; Koharudin, L. M.; Gronenborn, A. M.; Wetzel, R., Kinetically competing huntingtin aggregation pathways control amyloid polymorphism and properties. Biochemistry 2012, 51 (13), 2706-16.

83. Thakur, A. K.; Jayaraman, M.; Mishra, R.; Thakur, M.; Chellgren, V. M.; Byeon, I. J.; Anjum, D. H.; Kodali, R.; Creamer, T. P.; Conway, J. F.; Gronenborn, A. M.; Wetzel, R., Polyglutamine disruption of the huntingtin exon $1 \mathrm{~N}$ terminus triggers a complex aggregation mechanism. Nat Struct Mol Biol 2009, 16 (4), 380-9.

84. Tam, S.; Spiess, C.; Auyeung, W.; Joachimiak, L.; Chen, B.; Poirier, M. A.; Frydman, J., The chaperonin TRiC blocks a huntingtin sequence element that promotes the conformational switch to aggregation. Nat Struct Mol Biol 2009, 16 (12), 1279-85. 
85. Wieprecht, T.; Apostolov, O.; Beyermann, M.; Seelig, J., Interaction of a mitochondrial presequence with lipid membranes: role of helix formation for membrane binding and perturbation. Biochemistry 2000, 39 (50), 15297-305. 


\section{Molecular dynamics simulations of Proteorhodopsin oligomers in lipid bilayer and micellar systems}

\section{Introduction}

Proteorhodopsins (PRs) are microbial membrane proteins which exist as an oligomer (usually pentamer or hexamer) in their native form ${ }^{1}$. PRs are found in bacteria and they function as a proton pump. The ion pumping triggers with a photon absorbed by retinal and a series of events drive the proton to the outside of the bacteria Figure 4.1) ${ }^{2}$. The process starts with the retinol conformational change from all-trans isomer to 13-cis upon the photon absorption. This causes the formation of a photointermediate stat for the protein which is known as the $\mathrm{K}$ state. In the next step of the photocycle, D97 receives the proton from the protonated Schiff base (PSB), resulting in the formation of $\mathrm{M}$ state (Figure 4.1 and Figure 4.2). The PSB linkage is a result of the covalent binding between retinol and a lysine amino acid. A proton istransferred from the proton-donor E108 to the deprotonated SB, this step forms the $\mathrm{N}$ state. The proton lost by $\mathrm{E} 108$ is replaced by a proton from solvent. This state is known as the $\mathrm{O}$ state (Figure 4.1) ${ }^{3}$. As $\mathrm{H} 75$ has a hydrogen bond with the D97, this interaction tunes the pKa value for the proton acceptor $^{4}$. 


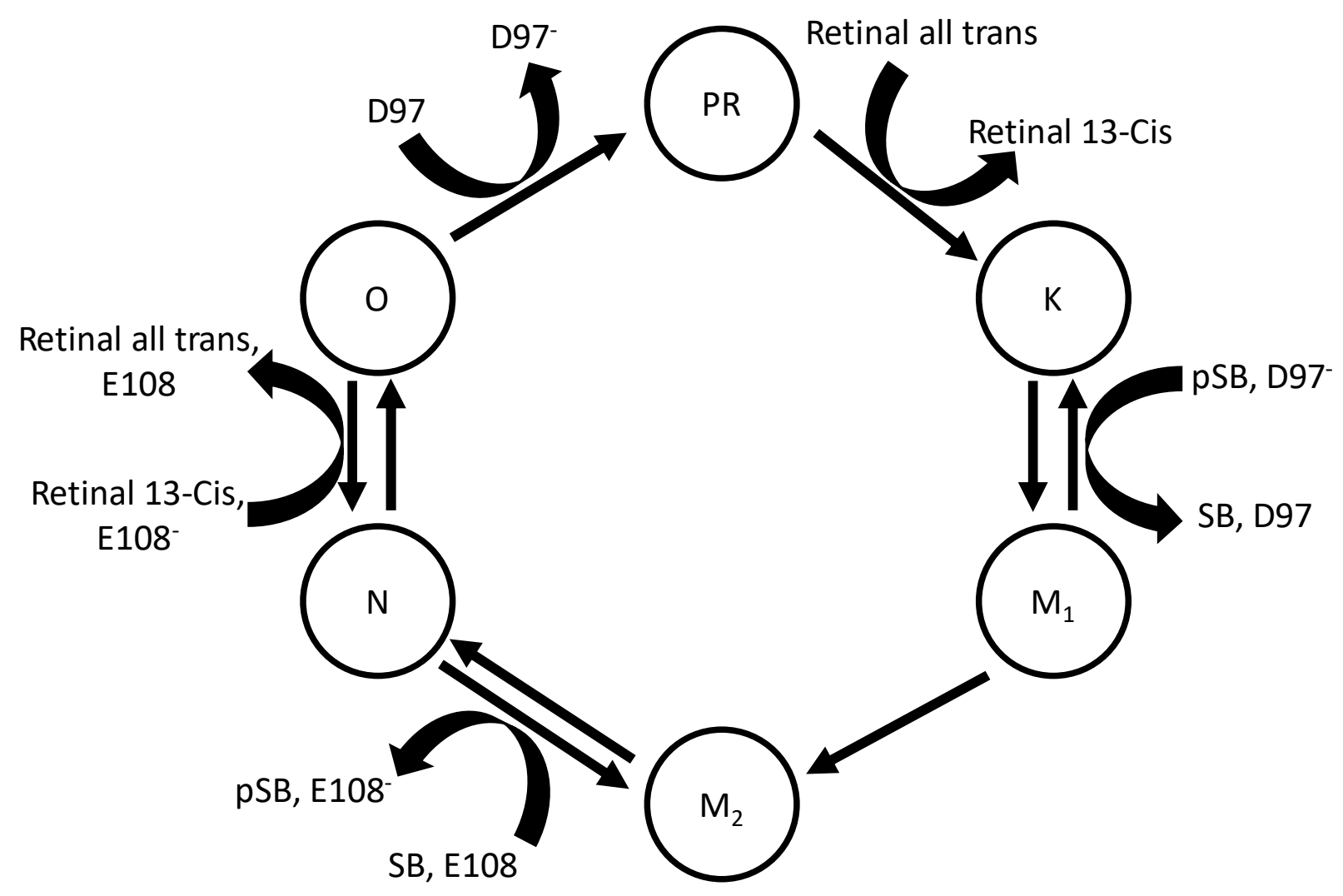

Figure 4. 1 Proteorhodopsin photocycle.

The photocycle starts with isomerization of the retinal molecule from all trans to 13-cis upon the absorption of a photon. The result of this photocycle is the transfer of a proton from the bacteria's cytoplasm to the periplasm.

PRs can be classified as green proteorhodopsin (GPR) ${ }^{5}$ or blue proteorhodopsin (BPR) according to the maximum light absorption ${ }^{6}$. PRs can form oligomeric states in order to increase the protein stability ${ }^{3-4,7-8}$. This oligomerization includes pentameric and hexametric forms of the protein ${ }^{9-11}$ (Figure 4.2). In the pentameric form, protein monomers such E50-R51 and D52-R51 salt bridges are specifically important for the protein stabilization ${ }^{7}$. In the hexameric form the corresponding residues in the BPR are E32-R33-S34-D35 in PDB 4JQ6. Maciejko, J. et al. have suggested that the presence of S34 will result in some degree of disruption in the R33-D35 salt bridge in the hexameric form therefore the pentameric oligomer is 
more favorable for the BPR ${ }^{7}$. The stability of a proteorhodopsin is also influenced by the membrane composition ${ }^{8,12}$. For example, the surface charge effect of the lipid bilayer on the recruitment as well as the orientation of PR in a detergent-mediated reconstitution procedure has been investigated by Noy and co-workers ${ }^{12}$. The effect of membrane system on the stability of oligomeric states of PR is also a subject of debate. It has been possible to capture the oligomeric PR within DDM ${ }^{10}$, and DPC ${ }^{8}$ micelles as well as various lipid bilayer systems ${ }^{7,}{ }^{138}$.

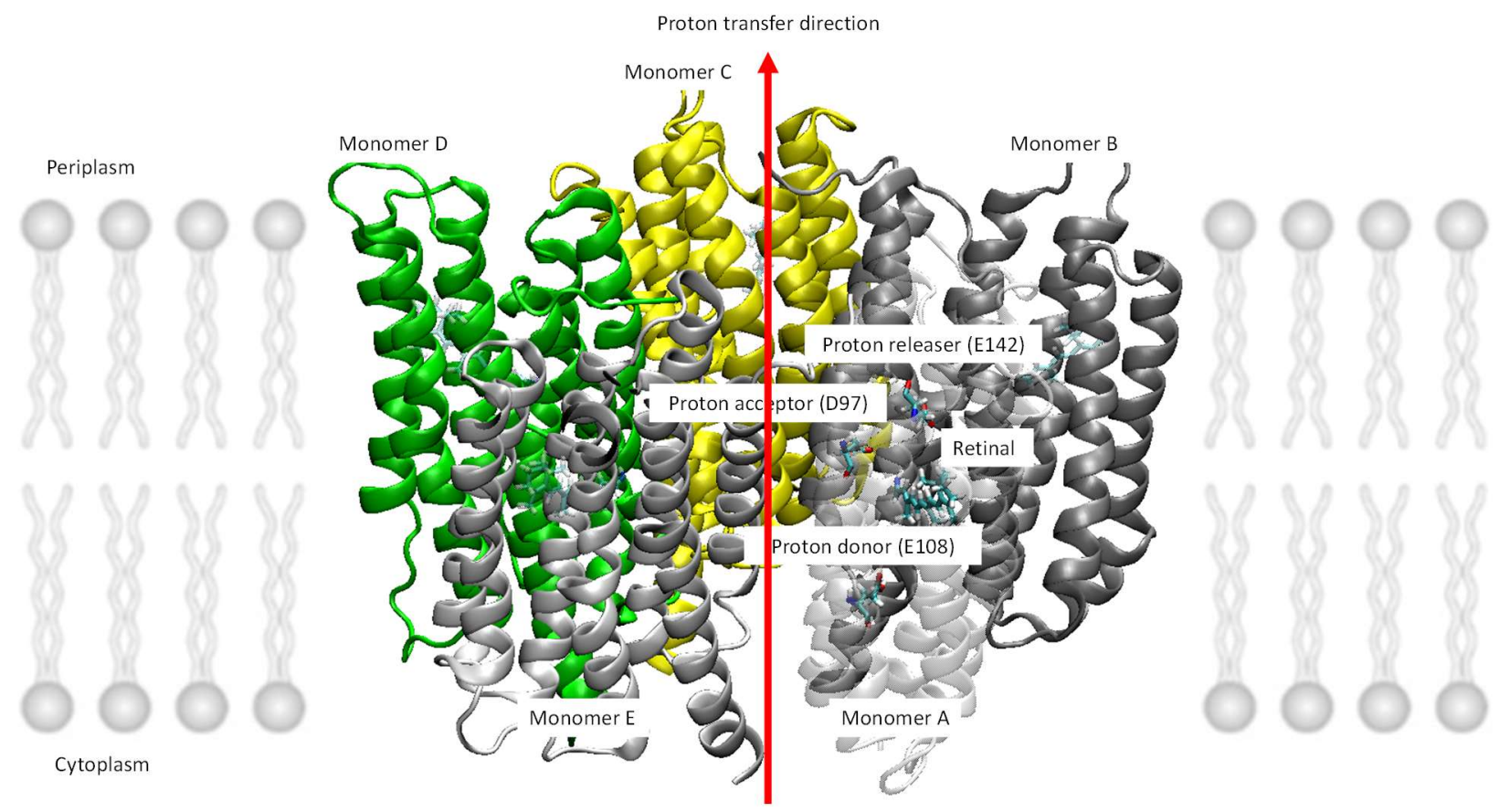

Figure 4. 2 Pentameric form of Proteorhodopsin.

The monomers are labeled as $A, B, C, D$ and $E$. The direction of the proton migration from cytoplasm to periplasm is shown with a red arrow. The proton donor (E108), retinal, proton acceptor (D97), and protein releaser (E142) residues are assigned only for the monomer A of the pentamer. The monomer $A$ is made transparent for better representation of the residues.

Our fundamental understanding of PR's structure and function is still not complete. The remaining questions include: 
1. How are the dynamic interactions between the monomers of an oligomeric PR?

2. What is the relation between the structural changes induced by variation of membrane environments on the PR interactions?

Molecular dynamics (MD) simulations can provide an atomistic insight into these events to reveal the details of the membrane composition effect on the photocycle as well as structural stability of PR.

Here we used Gaussian accelerated molecular dynamics (GaMD) ${ }^{14-15}$ simulations to investigate the effect of three detergent systems, n-dodecylphosphocholine (DPC), 1,2-dihexanoyl-sn-

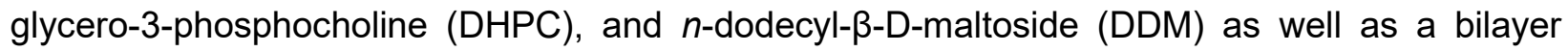
system 1-palmitoyl-2-oleoyl-sn-glycero-3-phosphoethanolamine (POPE): 1-palmitoyl-2-oleoylsn-glycero-3-phosphoglycerol (POPG) on the structure of PR in its oligomeric forms. Detergents and lipids can be classified as harsh and mild according to their ability to denature proteins ${ }^{16-17}$. In general, charged detergents with smaller head groups and shorter tails are classified as harsher detergents ${ }^{18}$. The important residues for oligomerization have also been monitored to study the oligomeric stability of PR.

\section{Computational Methods}

For the starting structure, the X-ray crystal structure of BPR from HOT75 and Med12 in the dark state (PDB 4KLY and 4JQ6) ${ }^{4}$ were used. Missing loops were modeled using MODELLER 19 based on the NMR structure of GPR (PDB 2L6X) ${ }^{20}$. The protonation states of the important residues in the dark sates are as follows: D97 deprotonated, E108 protonated, E142 protonated, H75 neutral with HSE protonated, all other titratable residues were in conventional protonation states. 
Details of the simulations can be found in Table 1. The CHARMM-GUI server (www.charmmgui.org) was used to create surfactant corona and lipid bilayer system around the protein ${ }^{21}$. In all cases we make sure that all the hydrophobic area of the protein is covered by detergents/lipid bilayer. The ionic strength of $100 \mathrm{mM} \mathrm{NaCl}$ was used in all the simulations.

All the systems were minimized for $1 \mathrm{ps}$, then heated up through 4 steps for 0.5 ns with 10 $\mathrm{kcal} / \mathrm{mol}$ constraints on the protein backbone. The minimization and heating were done with NAMD $2.13^{22}$ using CHARMM c36 force field for detergents/lipids and proteins ${ }^{23}$. After minimization, all the simulations were subjected to conventional molecular dynamics (cMD) simulation followed by Gaussian accelerated MD simulations implemented in NAMD ${ }^{15}$ with GPU acceleration and 2 fs time step. GaMD is a method that applies harmonic boost potential to enhance sampling by lowering the energy barriers in the $3 \mathrm{D}$ energy landscape of a biomolecule ${ }^{14-15,24}$. In GaMD there is no need to predefine collective variables (CVs) thus unconstrained enhanced sampling is achievable ${ }^{25}$. Smoothing the potential energy allows the MD simulation to cover the processing time of a biological system much faster. For example, a few hundred of nanosecond of a MD has been suggested to cover the biological processes that are in the millisecond time scale for membrane protein simulations ${ }^{14,26-28}$.

For the GaMD simulations, a dual boost potential was applied, to both the total potential energy and the dihedral terms. NPT ensemble was used for all the cMD and GaMD simulations at 310 $\mathrm{K}$ and 1 bar using a Langevin thermostat and a Berendsen barostat. Particle mesh Ewald (PME) summation was used to calculate the electrostatic interactions ${ }^{29}$. For non-bonded interactions, a force switching of $10 \AA$ and a cutoff of $12 \AA$ were utilized. Visual Molecular Dynamics (VMD) ${ }^{22}$ and LOOS ${ }^{30}$ were used to visualize and analyze the data. 
Table 4. 1 Simulation method and run times for all membrane systems.

\begin{tabular}{|c|c|c|c|c|c|c|c|c|}
\hline Membrane & Oligomer & \# atoms & $\begin{array}{c}\# \\
\text { detergents } \\
\text { or lipids }\end{array}$ & $\begin{array}{c}\text { lipid } \\
\text { (detergent) } \\
\text { per } \\
\text { monomer }\end{array}$ & $\begin{array}{l}\text { Waters / lipid } \\
\text { (detergent) }\end{array}$ & $\begin{array}{l}\text { CMD } \\
\text { (ns) }\end{array}$ & $\begin{array}{c}\text { GaMD } \\
\text { (ns) }\end{array}$ & $\begin{array}{l}\text { Total } \\
\text { (ns) }\end{array}$ \\
\hline \multirow{4}{*}{ DPC } & $P$ & 191917 & 380 & 76 & $50141 / 380=132$ & 77 & 320 & 397 \\
\hline & $P$ & 191917 & 380 & 76 & 132 & 99 & 328 & 429 \\
\hline & $\mathrm{H}$ & 326958 & 380 & 63 & $94050 / 380=247$ & 445 & 403 & 848 \\
\hline & $\mathrm{H}$ & 326958 & 380 & 63 & 247 & 140 & 342 & 482 \\
\hline \multirow{4}{*}{ DDM } & $P$ & 209823 & 350 & 70 & $54381 / 350=155$ & 54 & 320 & 374 \\
\hline & $P$ & 209823 & 350 & 70 & 155 & 57 & 346 & 403 \\
\hline & $\mathrm{H}$ & 357141 & 400 & 66 & $101029 / 400=252$ & 253 & 527 & 780 \\
\hline & $\mathrm{H}$ & 357141 & 400 & 66 & 252 & 362 & 306 & 668 \\
\hline \multirow{4}{*}{ DHPC } & $P$ & 196881 & 350 & 70 & $50655 / 350=144.7$ & 52 & 345 & 397 \\
\hline & $P$ & 196881 & 350 & 70 & 144.7 & 83 & 345 & 428 \\
\hline & $\mathrm{H}$ & 341878 & 380 & 63 & $97120 / 380=255$ & 414 & 308 & 722 \\
\hline & $\mathrm{H}$ & 341878 & 380 & 63 & 255 & 84 & 313 & 397 \\
\hline \multirow{8}{*}{ POPE:POPG } & $P$ & 244212 & 827 & 165 & $40644 / 827=49$ & 53 & 311 & 364 \\
\hline & $P$ & 244212 & 827 & 165 & 49 & 52 & 323 & 375 \\
\hline & D52A-P & 244202 & 827 & 165 & 49 & 37 & 117 & 154 \\
\hline & R51A-P & 244142 & 827 & 165 & 49 & 37 & 117 & 154 \\
\hline & $\mathrm{H}$ & 289929 & 808 & 134 & $55613 / 808=69$ & 353 & 1165 & 1518 \\
\hline & $\mathrm{H}$ & 289929 & 808 & 134 & 69 & 314 & 1019 & 1333 \\
\hline & D52A-H & 289905 & 808 & 134 & 69 & 33 & 102 & 135 \\
\hline & R51A-H & 289833 & 808 & 134 & 69 & 33 & 103 & 136 \\
\hline
\end{tabular}

For the simplicity, $\mathrm{H}$ and $\mathrm{P}$ were used as hexamer and pentamer respectively. Since each system were simulated in two copies, we were labeling them by 1 and 2 . In many cases terms like LB-H1 were used which means the lipid bilayer-hexamer system and copy 1 was the 
subject. As another example DDM-P2 means the environment is DDM, the protein is in pentameric form and the copy 2 was discussed.

\section{Results and Discussion}

Stability of PR oligomeric complexes. intra-protein contacts often lead to a more stable protein ${ }^{31}$. In the case of PR, it is suggested that forming oligomeric structure is intrinsic ${ }^{32}$ because the protein reconstitutes in different membrane environments ${ }^{33-34}$. Protein packing is also been suggested as another mechanism by which proteins gain stability ${ }^{35-38}$. For example, Fleming and co-workers showed that membrane proteins have higher packing values than soluble protein, indicating the helix-packing is an important factor for the stability of membrane proteins ${ }^{38}$. This packing is mainly due to the transmembrane (TM) helix-helix interfaces. This tool could be used to measure the efficiency of packing between monomers in an oligomeric complex. In the present work, the pentameric and hexameric forms of PR were used in different membrane environments (lipid bilayer, DDM, DHPC and DPC) in the dark sate. To investigate the oligomeric state stability of PR in different membrane systems, the packing score ${ }^{39}$ analysis was performed. The hypothesis is that denser TM bundle enabling PR to proceed more efficiently through photocycle. This can be explained by assuming that a closer interaction between the key residues helps the photocycle to perform faster by stabilizing the protein more efficiently. Figure 4.3 shows the packing score probability of hexameric and pentameric forms of PR scaled according to the environment composition. This figure shows that the protein is generally stable in the timeframe and all the environments that this study was performed in. 

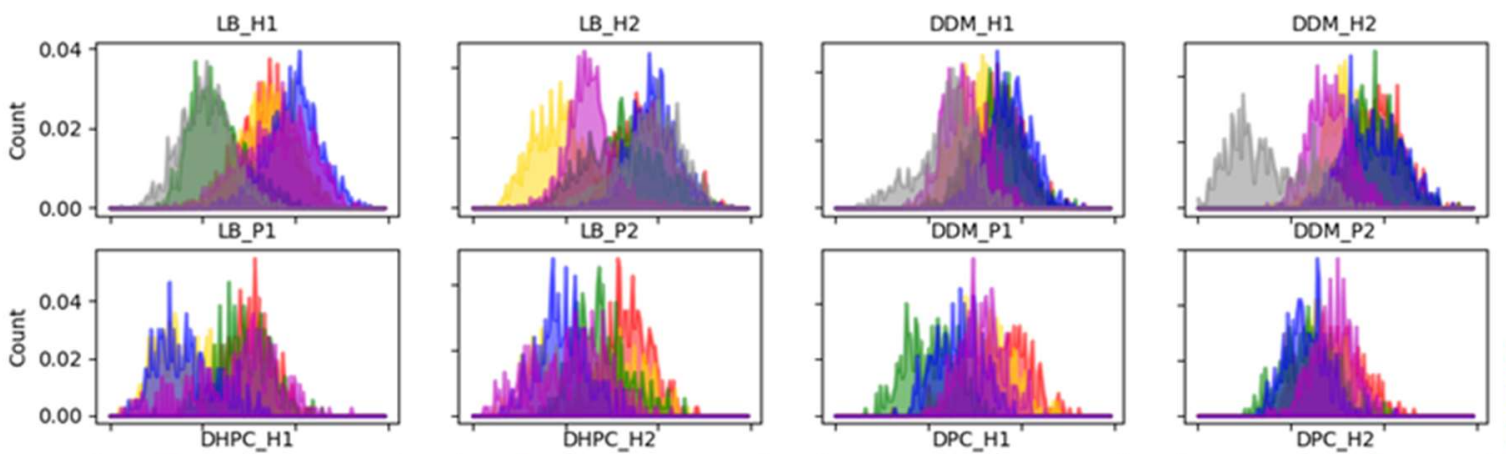

A-B
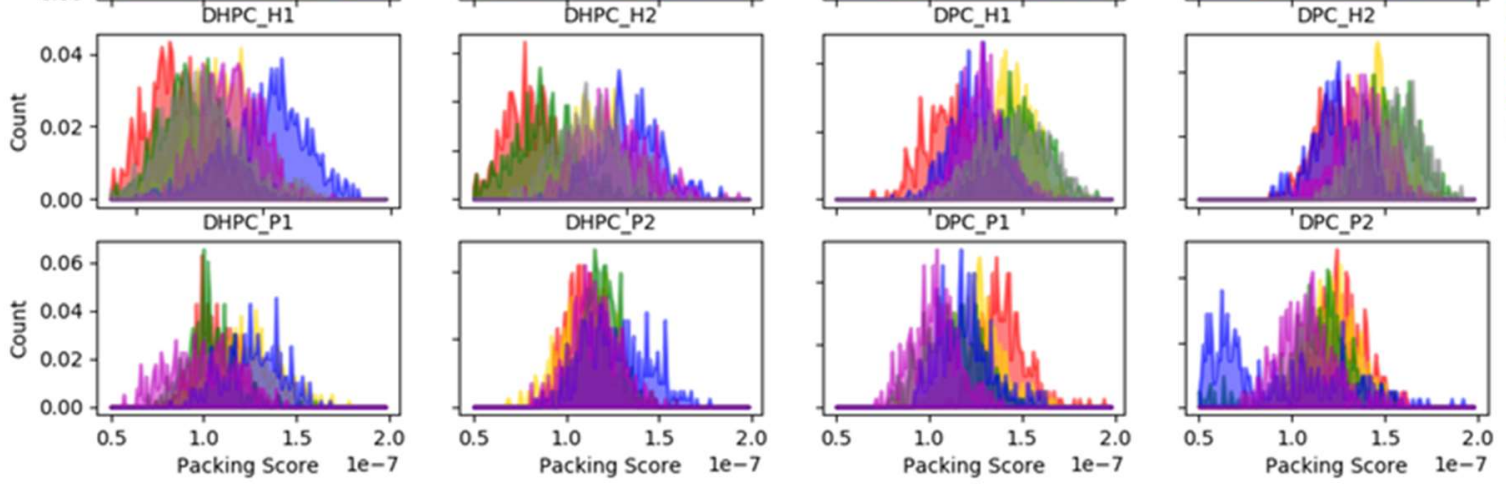

$B-C$
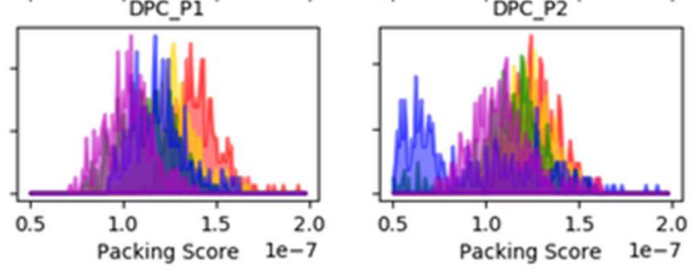

Figure 4. 3 Packing score as measured for all oligomeric systems.

LB = lipid bilayer; DDM = dodecyl maltoside; DHPC: diC7-PC; DPC: dodecylphosphocholine. $\mathrm{H}=\mathrm{Hexamer}$ and $\mathrm{P}=$ Pentamer. The number 1 and 2 after each system represents the two copy runs for each system. $\mathrm{A}-\mathrm{B}=$ monomer $\mathrm{A}-$ monomer $\mathrm{B}, \mathrm{B}-\mathrm{C}=$ monomer $\mathrm{B}-$ monomer $\mathrm{C}, \mathrm{C}-\mathrm{D}=$ monomer $\mathrm{C}-$ monomer $\mathrm{D}, \mathrm{D}-\mathrm{E}=$ monomer $\mathrm{D}-$ monomer $\mathrm{E}, \mathrm{E}-\mathrm{F}=$ monomer $\mathrm{E}-$ monomer $\mathrm{F}$, $\mathrm{F}-\mathrm{A}=$ monomer $\mathrm{F}-$ monomer $\mathrm{A}$. Worth to mention that in the pentameric system the magenta color is for $\mathrm{E}-\mathrm{A}=$ monomer $\mathrm{E}-$ monomer $\mathrm{A}$. The is no F-A interface in the pentameric systems.

However, hexameric form of the protein in the lipid bilayer is the only system that has 2 symmetric less-packed interfaces in both the copy runs. In LB-H1 system, the interfaces between C-D and F-A monomers are less packed in comparison to the other four interfaces. For LB-H2, there are a relatively less packing at the interfaces between B-C and E-F monomers. It can be inferred that according to the simulation data in the case of the dissociation of the hexameric system, there will be 2 trimer oligomers in the lipid bilayer environment. For the PRDHPC system, the pentameric form of the protein tend to be more uniformly grouped than 
hexamers. Nevertheless, the hexameric form have higher (more efficient) packing in the PRDPC system.

To further analyze the oligomeric tightness of PR protein, the distance between the center of masses of the adjacent heptameric TM bundles was measured. Figure 4.4 shows that there is a clear increase in the average distance (about 2-4 $\AA$ ) from hexameric to pentameric oligomer of PR. These results suggest that overall hexameric system pack more tightly than pentamers. This in general tracks the trends of the packing scores. Notably, for the lipid bilayer systems, the less packed interfaces have longer center of mass distances (C-D and F-A interfaces in LB-H1 and $\mathrm{B}-\mathrm{C}$ and $\mathrm{E}-\mathrm{F}$ interfaces in $\mathrm{LB}-\mathrm{H} 2)$. In another example, the F-A interface in the DDM-H2 system has the lowest packing score, that appears to have the longest center of mass distance. The same pattern can be applied for the D-E interfaces in DDM-P2 and DPC-P2 systems. It is essential to mention that the center of mass distance is not necessarily equivalent to the packing score. While the distance shows how far the center of masses are, the packing score reflects the short-distance interfaces (such as salt bridges) which by definition of packing score is a sum over a very short-ranged function $\left(\frac{1}{r^{6}}\right)$. As it is suggested by Glaubitz et al. ${ }^{7}$ the beltlike salt-bridges (D52-R51 and E50-R51), that are located at the top part of the cytoplasmicinteracting site of $\mathrm{PR}$, stabilize the oligomeric forms of PR. While the packing score analysis reflects the short distance interactions the movements of the rest of the protein causes the center of mass distances different from the packing score plots. 

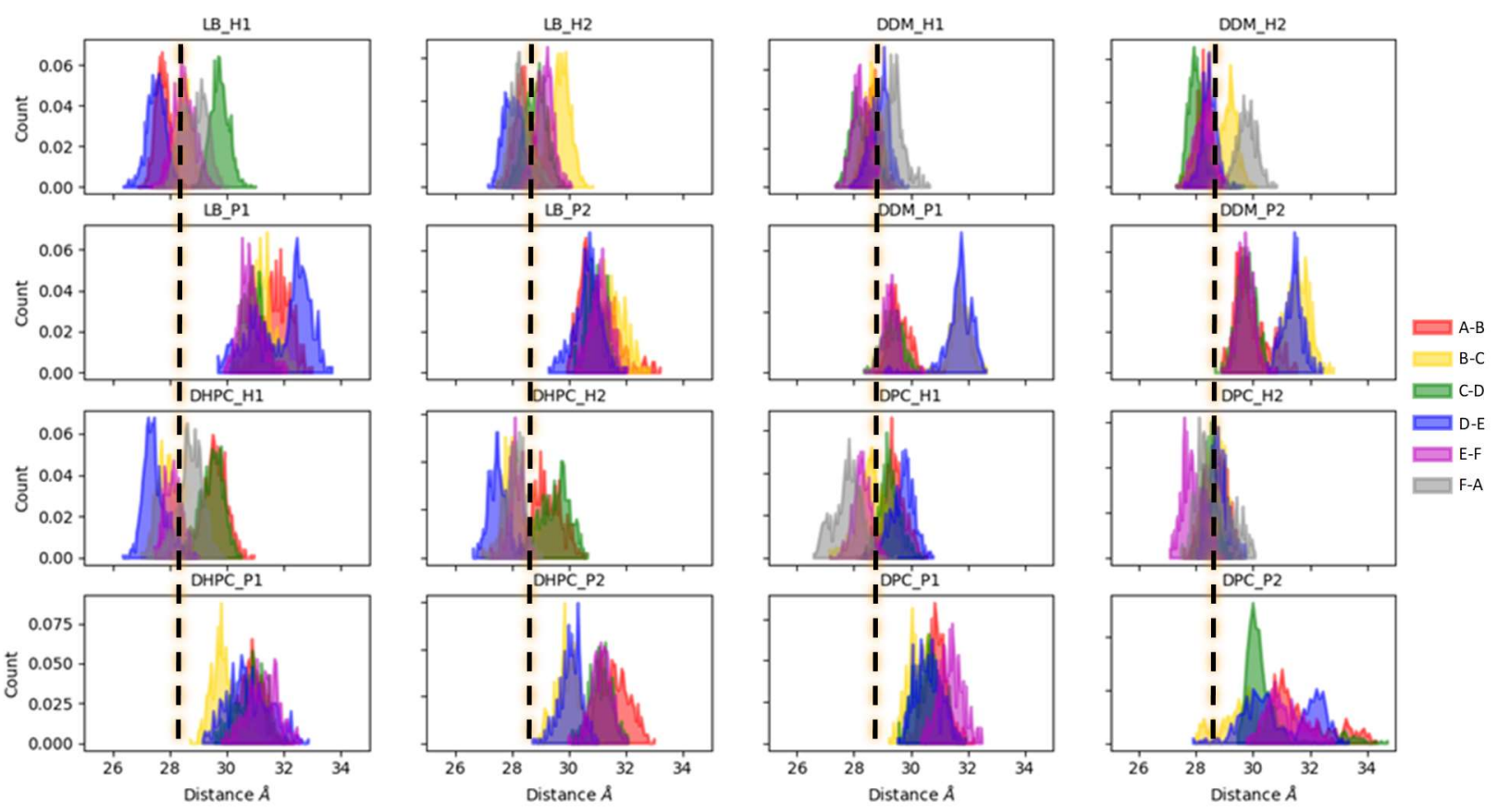

Figure 4. 4 Monomer-monomer distances between helix bundles: lipid bilayer vs. detergents.

Cross protomer interactions. As mentioned before E50-R51-D52 salt bridges play an important role in the oligomerization and stability of PR oligomers. It has been suggested that breaking the E50-R51 salt-bridge causes a "monomer switch" ${ }^{7}$ for PR. Figure 4.5 shows the distance probability of the interaction at this interface. The distance distribution is generally more stable for the hexamer than pentamer in all the four studied systems. The salt bridge distance is $\sim 4 \AA$, however, in some cases the salt bridge is broken and the distribution shifts to 6-7 A. For instance, the interface between monomer $E$ and $F$ and $B-C$ in $\mathrm{LB}-\mathrm{H} 2$ system is broken at some point. The probability of breaking this interaction is also noticeable in some pentamer systems such as in DDM-P and DPC-P. 

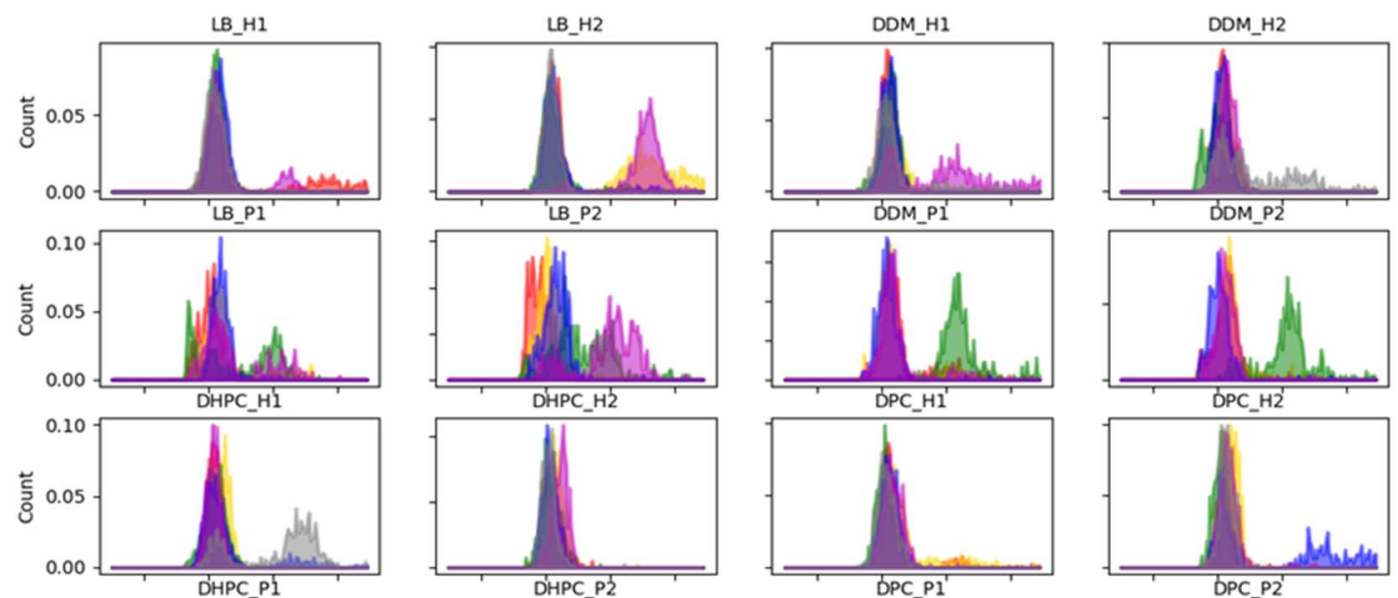

E50 A-R51 B E50 B-R51 C E50 C-R51 D

$\square$ E50 D-R51 E

$\square$ E50 E-R51 F
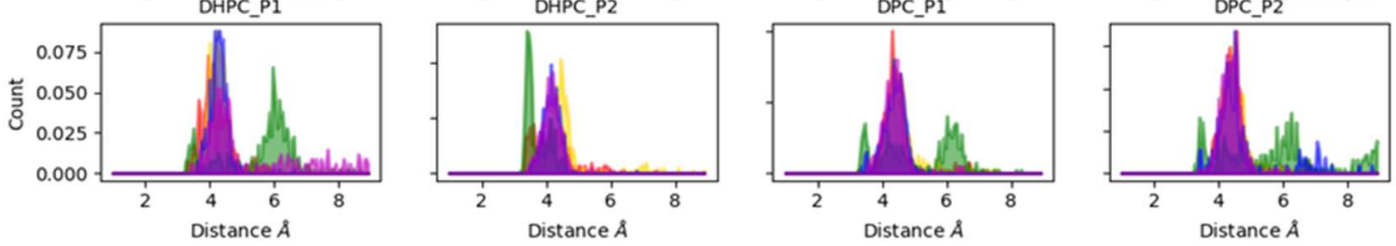

Figure 4. 5 Salt bridge distances between E50-R51.

Another important salt bridge is between D52 and R51. Figure 4.6 shows the distribution of this interaction for the PR protein in different environments. Compared to the E50-R51 salt bridge, D52-R51 interaction is more stable. Except for the LB-H system in which there is a notable higher distance for the other systems only a small tail was observed. Furthermore, a shorter distance between the D52-R51 than E50-R51 was observed especially for the pentameric systems.

Generally, it is essential to know which salt bridge is stronger in the PR to control the monomeroligomer population. A desired population of monomer can be obtained by mutating the correct residue in a pentameric or hexameric systems in different environments. This work can guide through new mutations in order to control the monomer-oligomer populations.

It worth mentioning that the salt bridges are fundamentally different in hexamer and pentamer forms of PR. While in the pentameric system form the sequence is regular E50-R51-D52, in the hexameric form there is an additional residue (serine) between R51 and D52 which affects the 
stability of the salt bridge triad in the hexamer. This is most likely why there is a longer distance between D52-R51 in hexamer compare to the same distance in pentamer.

Also, a 2D plot relation between the R51 X1 dihedral angle and D52-R51 and E50-R51 distances shows that weaker packing from detergent environments allows E50-R51-D52 to interact more closely, specifically in that +/- 60-degree orientation. As such, DHPC systems are the only ones that noticeably sample R51 dihedral angle at $+/-60$ that are combined with short enough distance to form a salt bridge. In comparison to hexameric system in DHPC (Figure S4.3 (Appendix C)), the pentameric system in DHPC (Figure S4.4 (Appendix C)) demonstrate a definite shift in the population of the R51 dihedral which causes this system to have much larger percentages of simulation time in +/- 60 deg orientation, coupled with tighter binding to E50/D52. Part of this is attributable to the additional residue in the hexamer system (as mentioned before). In this case, membrane environment is not as important, rather the oligomeric form and the position of salt bridge triad is critical. (compare Figure S4.3 (Appendix C) and Figure S4. 4 (Appendix C)).

To further investigate the effect of E50-R51-D52 salt-bridges on the protein packing score the mutation of D52 and R51 to alanine were performed separately for both the oligomer systems in the lipid bilayer environment. Figure S4. 1 (Appendix C) represents the packing score analysis for the wildtype PR in the lipid bilayer system versus mutated version of the protein in the same environment. The analysis reveals that the mutation of either D52 or R51 leads to weaker oligomeric bundle packing. This is more pronounced weakening of the interface for R51A. The center of mass analysis shows that the mutations lead to slight increase in hexamers but have no effect or decrease in pentameric systems (Figure S4. 2 (Appendix C)). 

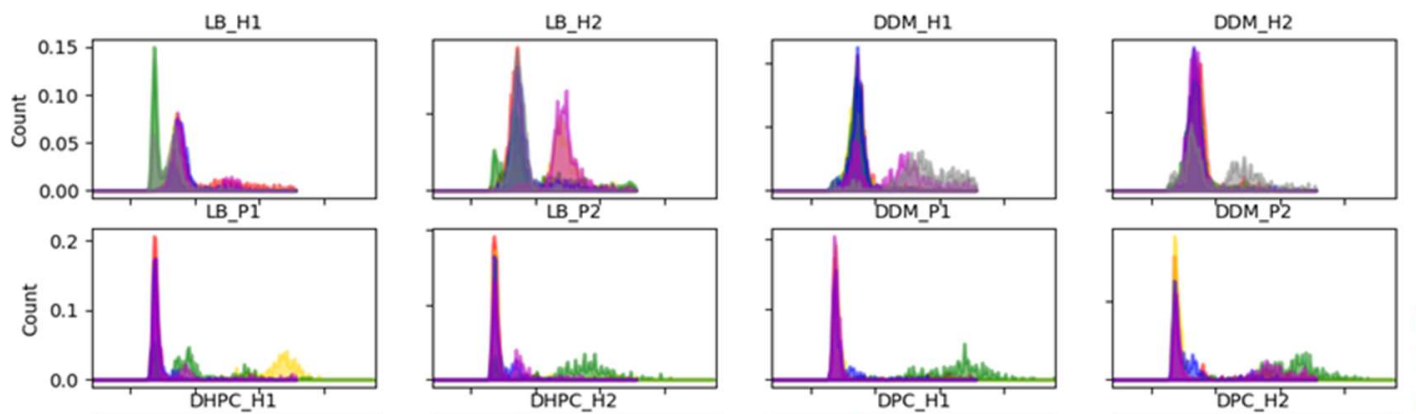

D52 A-R51B
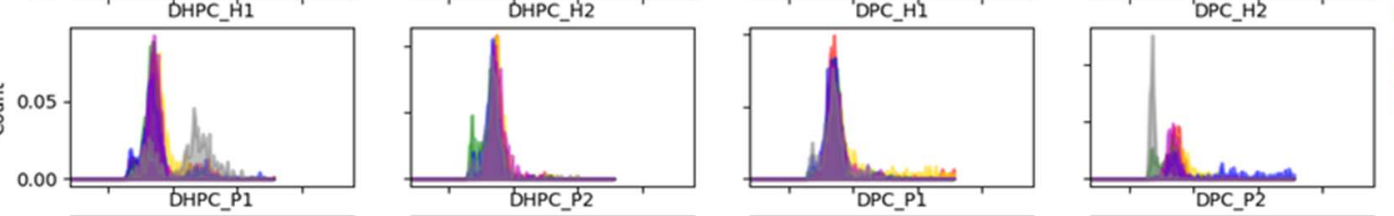

D52 B-R51 C D52 C-R51 D

$\square$ D52 D-R51 E

$\square$ D52 E-R51 F
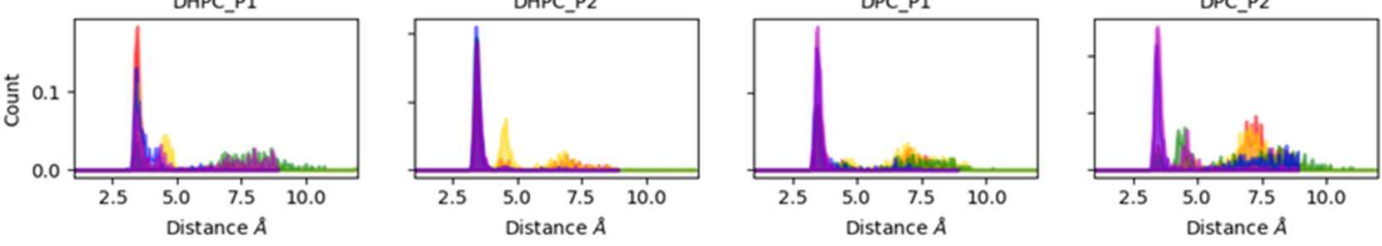

Figure 4. 6 Salt bridge distances between D52-R51.

Other than the two well known cross protomer interactions in the PR oligomers, the salt bridge between D52 and K59 at the ajdacent monomers are also important. Figure 4.7 depicts a strong indications between D52-K59 salt bridge. This interaction is more of a factor in stabilization of the hexamer vs. pentamer, regardless of membrane environment. In all lipid bilayer and detergent enviromets a close interaction between D52-K59 is centered around $<4 \AA$ for hexameric systems that is much more stable than the same interaction for the pentameric systems in which the distrubution is more expanded. In most of the cases in the pentamer systems there is at least one interface that are close to each aother (have a distance $<4 \AA$ ) but this not consitent for the other interfaces. For example the interface between monomers D-E in DDM-P, DHPC-P, and DPC-P is close but the other interfaces are not as close as the interface between $\mathrm{D}$ and $\mathrm{E}$. 

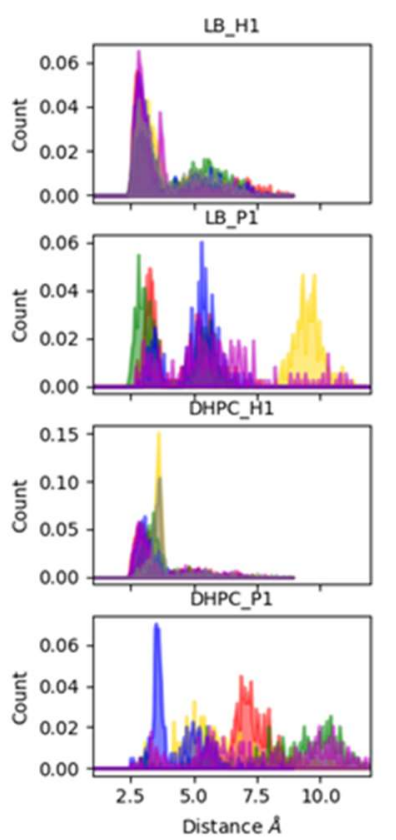

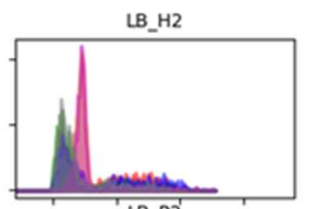

LB_P2'

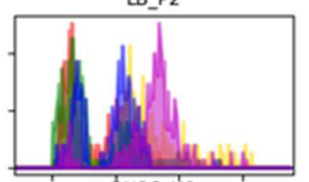

BHPC_H2
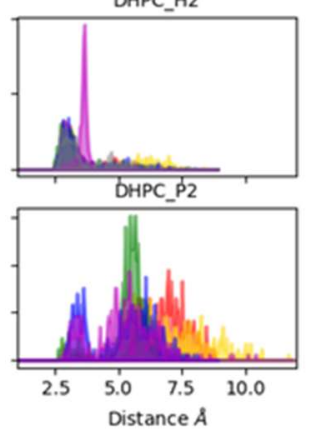

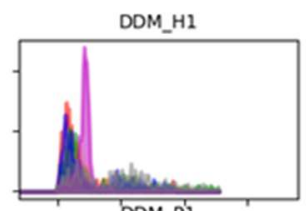

DDM_P1

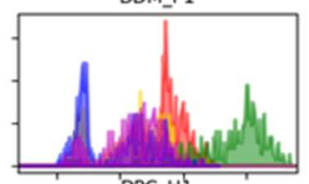

'DPC_Hl
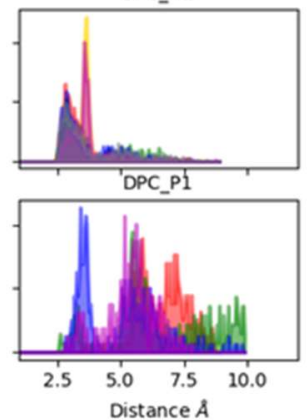

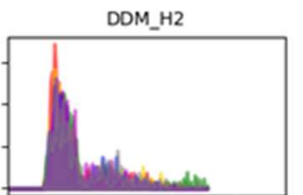

DDM_P2

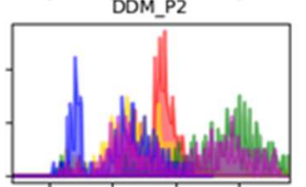

'DPC_H2
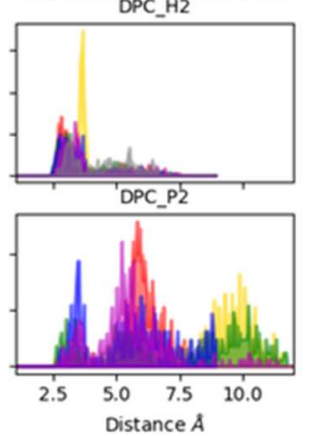

D52 B-K59 C D52 C-K59 D D52 D-K59 E D52 E-K59 F D52 F-K59 A

Figure 4. 7 Salt bridge distances between D52-K59.

W34-H75 interaction. Because of the effect of the $\mathrm{H} 75$ on the pKa value of the proton acceptor D97 investigating the hydrogen bond between W34 and H75 is important ${ }^{40-41}$. The $\mathrm{H} 75$ distance analysis reveals that while there is a distribution of distances from 2.5-7.5 $\AA$, regardless of the membrane system for H75-D97 interaction (Figure S4. 5 (Appendix C)), the W34-H75 distance is much more pronounced at extremely short distances at $<2.5 \AA$ (Figure 4. 8). Also, for all the studied systems there is a peak that is centered $\sim 4 \AA$ which is still a strong interaction between W34 and H75. In some cases, in some of the monomers the distance increases to $>5 \AA$ (Figure 4. 6). Furthermore, the $X^{1}$ dihedral angle analysis of $\mathrm{H} 75$ reveals that this residue adopts 2 conformations in the pentamer (-60 and 180) versus one conformation in the hexamer (-60), but not directly related to interactions with either D97 or W34 (Figure S4. 6 (Appendix C) and Figure S4. 7 (Appendix C)). As it is suggested by Luecke and coworkers 
W34D mutation causes a drastic alteration in the BPR function ${ }^{4}$ the close interaction of W34 and $\mathrm{H} 75$ is playing an important role in the photochemical activity of PR.
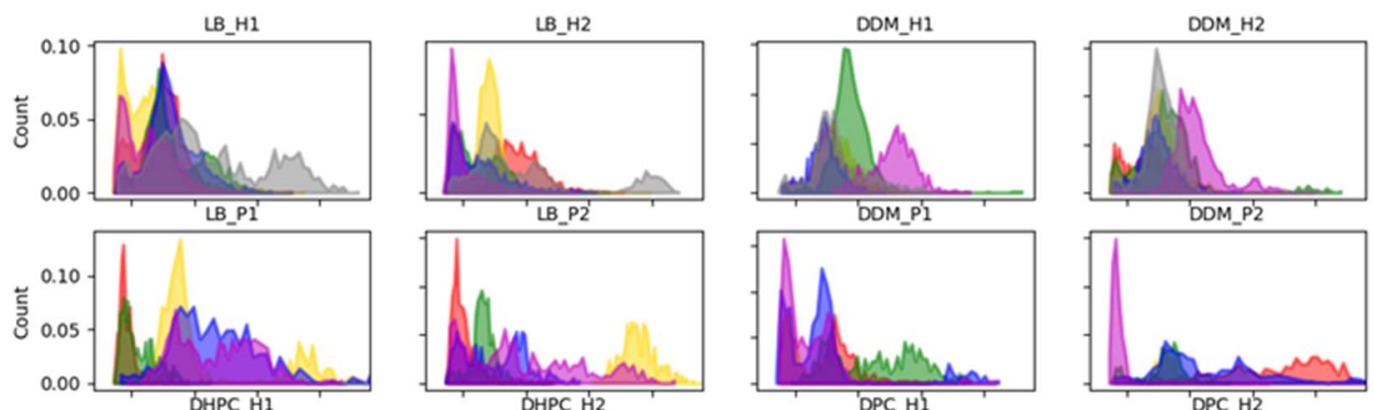

W34 A-H75B
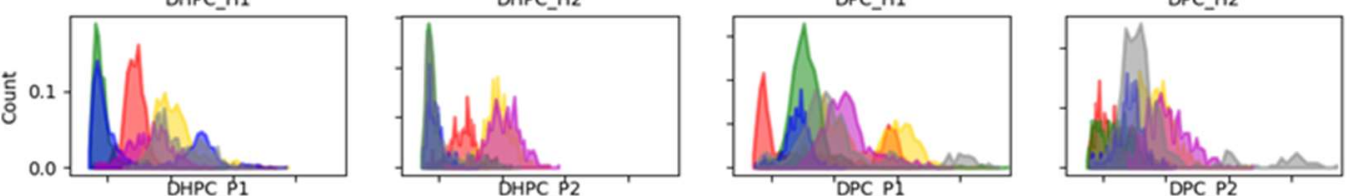

W34 B-H75 C
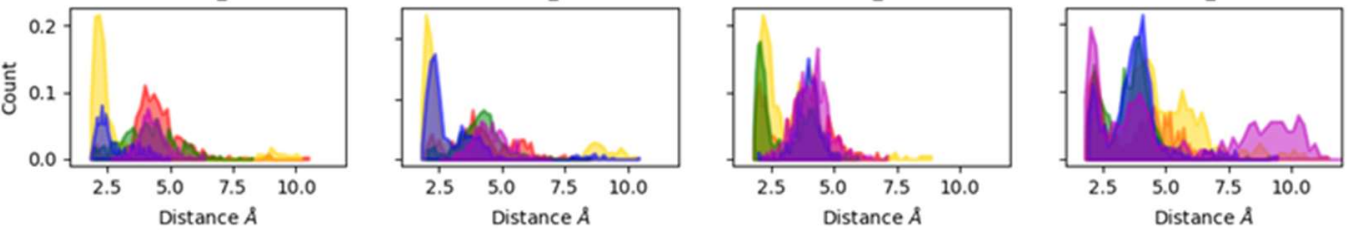

Figure 4. 8 Hydrogen bond between W34 and H75.

Retinal binding pocket. One initial step to help with photoisomerization is to transfer a $\mathrm{H}+$ from the protonated schiff base (PSB) on the retinal to the proton acceptor D97. The pKa value of the PSB is affected by the localized changes in the binding pocket. Two important residues that the PSB is interacting with are D97 and D227 that according to the x-ray structure of PR the D227 is in closer interaction with the $\mathrm{PSB}^{4}$. D227 is important because retinal photoisomerization selectivity is controlled by this residue ${ }^{42}$. Also, it is been shown that D227 has a critical role in modulating the pKa value for the PSB and therefore the rate of the photocycle of proteorhodopsin ${ }^{43}$. Figure 4. 9 and Figure 4. 10 show that in the hexamer system 
D227 is stabilizing the PSB in the all-trans conformation, while D97 is free to fluctuate. A close interaction ( $>4 \AA$ ) between the NZ atoms of the D227 with the NZ atom at the retinal in all the hexamer systems is shown in Figure 4. 9. The same analysis for the distance between D97 and retinal in Figure 4. 10 shows a broad distribution of probabilities for the D97-retinal distance in the hexamer systems. This is indicating that only after isomerization the D97 residue is close enough to the PSB to facilitate proton transfer characteristic of the $\mathrm{K}$ to $\mathrm{M}$ transition.

Conversely in the pentameric system, D97 is (potentially) bridged with water molecule to PSB, allowing it to be more stable (Figure 4. 10). In contrast, D227 is more distant, but only for a subset of monomers (Figure 4. 9).
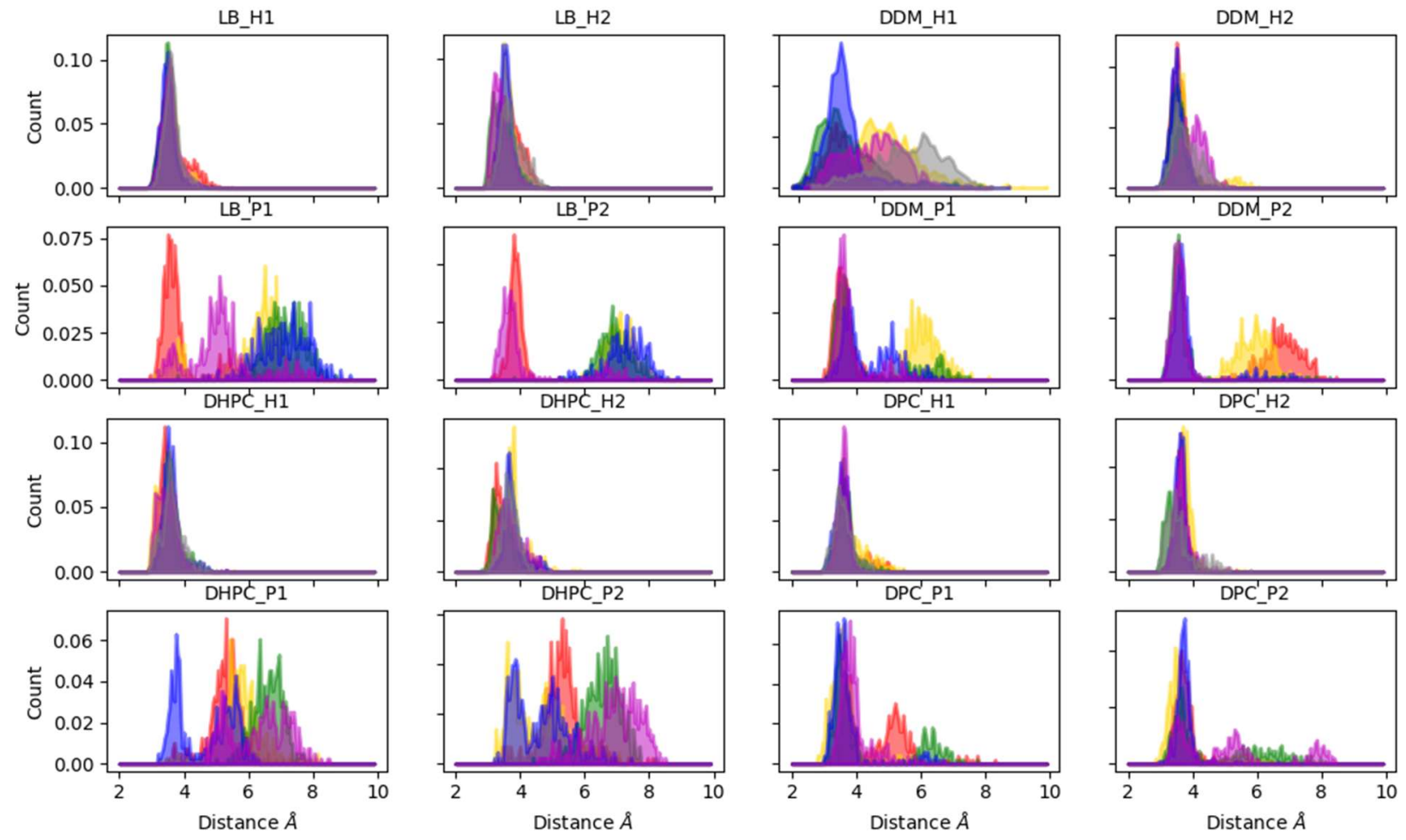

Figure 4. 9 Retinal binding pocket: Schiff base to D227. 

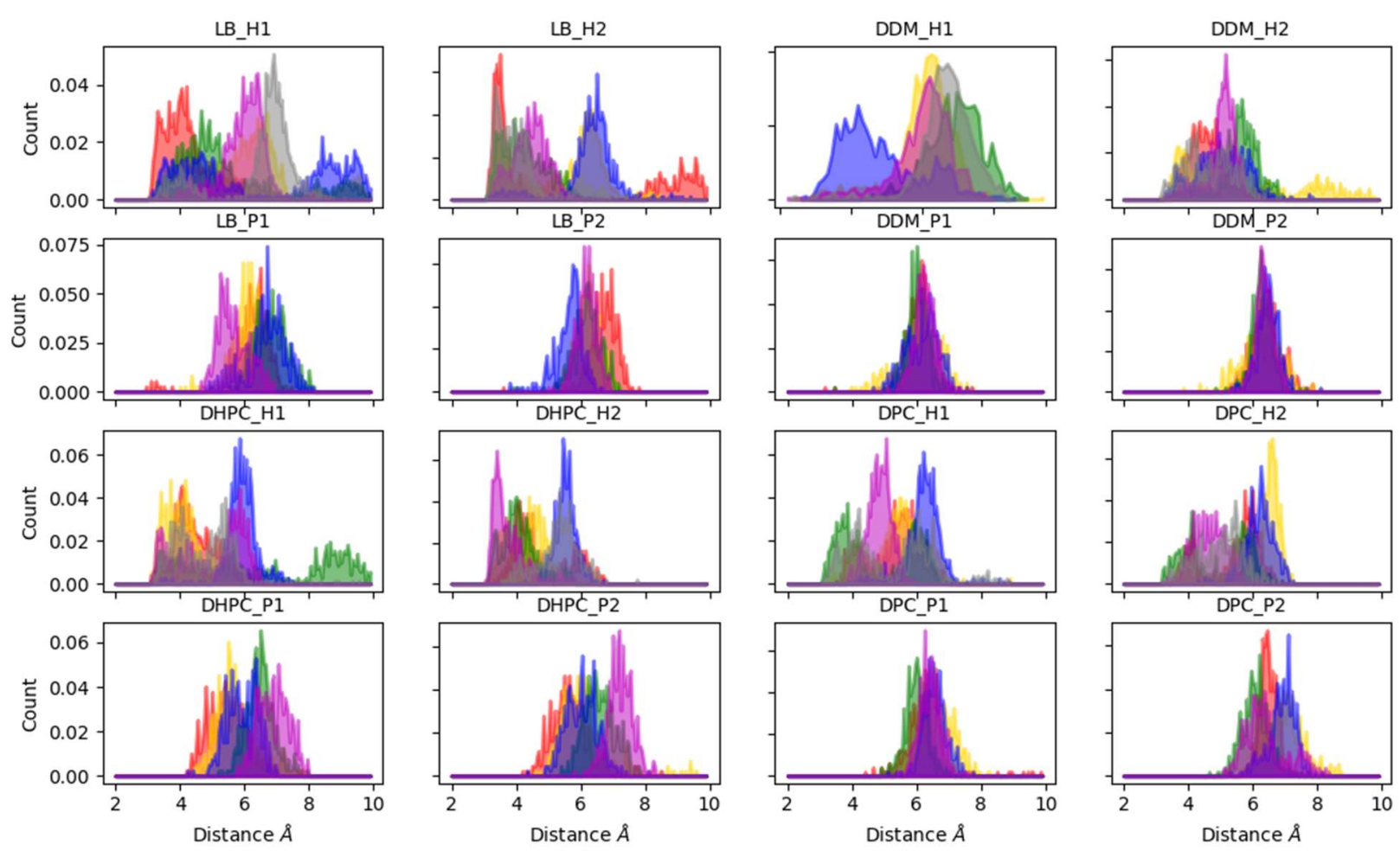

Figure 4. 10 Retinal binding pocket: Schiff base to D97.

\section{Conclusion}

Molecular dynamics simulations were used to study proteorhodopsin protein in its oligomeric (pentameric and hexameric) form in four different membrane environments. To study the stability of PR the packing score and salt bridge analysis were performed. The packing score and center of chain distance analysis showed that the PR is stable in both the pentameric and hexameric form in all four membrane systems in the simulation time scale. The distance analysis of important salt bridges also reveals that D52-R51 is especially important in the pentameric system. Another salt bridge that is obviously strong in the hexameric system and less pronounce in the pentameric system was the D52-K59 interaction. The W34 interactions were also studied to investigate the importance of the W34-H75 interaction in the photochemical 
activity of proteorhodopsin. Finally, the interaction between the PSB and close aspartic acid residues were studied.

\section{References}

1. Bamann, C.; Bamberg, E.; Wachtveitl, J.; Glaubitz, C., Proteorhodopsin. Biochim Biophys Acta 2014, 1837 (5), 614-25.

2. Friedrich, T.; Geibel, S.; Kalmbach, R.; Chizhov, I.; Ataka, K.; Heberle, J.; Engelhard, M.; Bamberg, E., Proteorhodopsin is a Light-driven Proton Pump with Variable Vectoriality. Journal of Molecular Biology 2002, 321 (5), 821-838.

3. Maciejko, J.; Kaur, J.; Becker-Baldus, J.; Glaubitz, C., Photocycle-dependent conformational changes in the proteorhodopsin cross-protomer Asp-His-Trp triad revealed by DNP-enhanced MASNMR. Proc Natl Acad Sci U S A 2019, 116 (17), 8342-8349.

4. Ran, T.; Ozorowski, G.; Gao, Y.; Sineshchekov, O. A.; Wang, W.; Spudich, J. L.; Luecke, H., Crossprotomer interaction with the photoactive site in oligomeric proteorhodopsin complexes. Acta Crystallogr D Biol Crystallogr 2013, 69 (Pt 10), 1965-80.

5. Albarracin, V. H.; Kraiselburd, I.; Bamann, C.; Wood, P. G.; Bamberg, E.; Farias, M. E.; Gartner, W., Functional Green-Tuned Proteorhodopsin from Modern Stromatolites. PLoS One 2016, 11 (5), e0154962.

6. Yamada, K.; Kawanabe, A.; Yoshizawa, S.; Inoue, K.; Kogure, K.; Kandori, H., Anomalous pH Effect of Blue Proteorhodopsin. J Phys Chem Lett 2012, 3 (7), 800-4.

7. Maciejko, J.; Mehler, M.; Kaur, J.; Lieblein, T.; Morgner, N.; Ouari, O.; Tordo, P.; Becker-Baldus, J.; Glaubitz, C., Visualizing Specific Cross-Protomer Interactions in the Homo-Oligomeric Membrane Protein Proteorhodopsin by Dynamic-Nuclear-Polarization-Enhanced Solid-State NMR. J Am Chem Soc 2015, 137 (28), 9032-43.

8. Hussain, S.; Kinnebrew, M.; Schonenbach, N. S.; Aye, E.; Han, S., Functional consequences of the oligomeric assembly of proteorhodopsin. J Mol Biol 2015, 427 (6 Pt B), 1278-1290.

9. Edwards, D. T.; Huber, T.; Hussain, S.; Stone, K. M.; Kinnebrew, M.; Kaminker, I.; Matalon, E.; Sherwin, M. S.; Goldfarb, D.; Han, S., Determining the oligomeric structure of proteorhodopsin by Gd3+ based pulsed dipolar spectroscopy of multiple distances. Structure 2014, 22 (11), 1677-86.

10. Stone, K. M.; Voska, J.; Kinnebrew, M.; Pavlova, A.; Junk, M. J.; Han, S., Structural insight into proteorhodopsin oligomers. Biophys J 2013, 104 (2), 472-81.

11. Gourdon, P.; Alfredsson, A.; Pedersen, A.; Malmerberg, E.; Nyblom, M.; Widell, M.; Berntsson, R.; Pinhassi, J.; Braiman, M.; Hansson, O.; Bonander, N.; Karlsson, G.; Neutze, R., Optimized in vitro and in vivo expression of proteorhodopsin: a seven-transmembrane proton pump. Protein Expr Purif 2008, $58(1), 103-13$.

12. Tunuguntla, R.; Bangar, M.; Kim, K.; Stroeve, P.; Ajo-Franklin, C. M.; Noy, A., Lipid bilayer composition can influence the orientation of proteorhodopsin in artificial membranes. Biophys $J$ 2013, 105 (6), 1388-96.

13. Lindholm, L.; Arioz, C.; Jawurek, M.; Liebau, J.; Maler, L.; Wieslander, A.; von Ballmoos, C.; Barth, A., Effect of lipid bilayer properties on the photocycle of green proteorhodopsin. Biochim Biophys Acta 2015, 1847 (8), 698-708. 
14. Miao, Y.; Feher, V. A.; McCammon, J. A., Gaussian Accelerated Molecular Dynamics: Unconstrained Enhanced Sampling and Free Energy Calculation. J Chem Theory Comput 2015, 11 (8), 3584-3595.

15. Pang, Y. T.; Miao, Y.; Wang, Y.; McCammon, J. A., Gaussian Accelerated Molecular Dynamics in NAMD. J Chem Theory Comput 2017, 13 (1), 9-19.

16. Prive, G. G., Detergents for the stabilization and crystallization of membrane proteins. Methods 2007, 41 (4), 388-97.

17. Tate, C. G., Practical considerations of membrane protein instability during purification and crystallisation. Methods Mol Biol 2010, 601, 187-203.

18. Yang, Z.; Wang, C.; Zhou, Q.; An, J.; Hildebrandt, E.; Aleksandrov, L. A.; Kappes, J. C.; DeLucas, L. J.; Riordan, J. R.; Urbatsch, I. L.; Hunt, J. F.; Brouillette, C. G., Membrane protein stability can be compromised by detergent interactions with the extramembranous soluble domains. Protein Sci 2014, 23 (6), 769-89.

19. Webb, B.; Sali, A., Comparative Protein Structure Modeling Using MODELLER. Curr Protoc Bioinformatics 2016, 54, 56 1-5 637.

20. Reckel, S.; Gottstein, D.; Stehle, J.; Lohr, F.; Verhoefen, M. K.; Takeda, M.; Silvers, R.; Kainosho, M.; Glaubitz, C.; Wachtveitl, J.; Bernhard, F.; Schwalbe, H.; Guntert, P.; Dotsch, V., Solution NMR structure of proteorhodopsin. Angew Chem Int Ed Engl 2011, 50 (50), 11942-6.

21. Jo, S.; Kim, T.; Iyer, V. G.; Im, W., CHARMM-GUI: a web-based graphical user interface for CHARMM. J Comput Chem 2008, 29 (11), 1859-65.

22. Phillips, J. C.; Braun, R.; Wang, W.; Gumbart, J.; Tajkhorshid, E.; Villa, E.; Chipot, C.; Skeel, R. D.; Kale, L.; Schulten, K., Scalable molecular dynamics with NAMD. J Comput Chem 2005, 26 (16), 1781-802.

23. Vanommeslaeghe, K.; Hatcher, E.; Acharya, C.; Kundu, S.; Zhong, S.; Shim, J.; Darian, E.; Guvench, O.; Lopes, P.; Vorobyov, I.; Mackerell, A. D., Jr., CHARMM general force field: A force field for drug-like molecules compatible with the CHARMM all-atom additive biological force fields. I Comput Chem 2010, 31 (4), 671-90.

24. Bhattarai, A.; Miao, Y., Gaussian accelerated molecular dynamics for elucidation of drug pathways. Expert Opin Drug Discov 2018, 13 (11), 1055-1065.

25. Markwick, P. R.; McCammon, J. A., Studying functional dynamics in bio-molecules using accelerated molecular dynamics. Phys Chem Chem Phys 2011, 13 (45), 20053-65.

26. Miao, Y.; Caliman, A. D.; McCammon, J. A., Allosteric effects of sodium ion binding on activation of the $\mathrm{m} 3$ muscarinic g-protein-coupled receptor. Biophys J 2015, 108 (7), 1796-1806.

27. Miao, Y.; Nichols, S. E.; McCammon, J. A., Free energy landscape of G-protein coupled receptors, explored by accelerated molecular dynamics. Phys Chem Chem Phys 2014, 16 (14), 6398-406.

28. Pierce, L. C.; Salomon-Ferrer, R.; Augusto, F. d. O. C.; McCammon, J. A.; Walker, R. C., Routine Access to Millisecond Time Scale Events with Accelerated Molecular Dynamics. J Chem Theory Comput 2012, 8 (9), 2997-3002.

29. Essmann, U.; Perera, L.; Berkowitz, M. L.; Darden, T.; Lee, H.; Pedersen, L. G., A smooth particle mesh Ewald method. The Journal of Chemical Physics 1995, 103 (19), 8577-8593.

30. Romo, T. D.; Grossfield, A., LOOS: an extensible platform for the structural analysis of simulations. Conf Proc IEEE Eng Med Biol Soc 2009, 2009, 2332-5.

31. Engelman, D. M., Membranes are more mosaic than fluid. Nature 2005, 438 (7068), 578-80.

32. Klyszejko, A. L.; Shastri, S.; Mari, S. A.; Grubmuller, H.; Muller, D. J.; Glaubitz, C., Folding and assembly of proteorhodopsin. J Mol Biol 2008, 376 (1), 35-41.

33. Shastri, S.; Vonck, J.; Pfleger, N.; Haase, W.; Kuehlbrandt, W.; Glaubitz, C., Proteorhodopsin: characterisation of 2D crystals by electron microscopy and solid state NMR. Biochim Biophys Acta 2007, 1768 (12), 3012-9. 
34. Ranaghan, M. J.; Schwall, C. T.; Alder, N. N.; Birge, R. R., Green proteorhodopsin reconstituted into nanoscale phospholipid bilayers (nanodiscs) as photoactive monomers. J Am Chem Soc 2011, 133 (45), 18318-27.

35. DeDecker, B. S.; O'Brien, R.; Fleming, P. J.; Geiger, J. H.; Jackson, S. P.; Sigler, P. B., The crystal structure of a hyperthermophilic archaeal TATA-box binding protein. J Mol Biol 1996, 264 (5), 1072-84.

36. Bisht, M.; Mondal, D.; Pereira, M. M.; Freire, M. G.; Venkatesu, P.; Coutinho, J. A. P., Long-term protein packaging in bio-ionic liquids: Improved catalytic activity and enhanced stability of cytochrome $\mathrm{C}$ against multiple stresses. Green Chem 2017, 19 (20), 4900-4911.

37. Leygonie, C.; Britz, T. J.; Hoffman, L. C., Protein and lipid oxidative stability of fresh ostrich M. Iliofibularis packaged under different modified atmospheric packaging conditions. Food Chemistry 2011, 127 (4), 1659-1667.

38. Eilers, M.; Shekar, S. C.; Shieh, T.; Smith, S. O.; Fleming, P. J., Internal packing of helical membrane proteins. Proc Natl Acad Sci U S A 2000, 97 (11), 5796-801.

39. Grossfield, A.; Feller, S. E.; Pitman, M. C., A role for direct interactions in the modulation of rhodopsin by omega-3 polyunsaturated lipids. Proc Natl Acad Sci U S A 2006, 103 (13), 4888-93.

40. Hempelmann, F.; Holper, S.; Verhoefen, M. K.; Woerner, A. C.; Kohler, T.; Fiedler, S. A.; Pfleger, N.; Wachtveitl, J.; Glaubitz, C., His75-Asp97 cluster in green proteorhodopsin. J Am Chem Soc 2011, 133 (12), 4645-54.

41. Balashov, S. P.; Petrovskaya, L. E.; Lukashev, E. P.; Imasheva, E. S.; Dioumaev, A. K.; Wang, J. M.; Sychev, S. V.; Dolgikh, D. A.; Rubin, A. B.; Kirpichnikov, M. P.; Lanyi, J. K., Aspartate-histidine interaction in the retinal schiff base counterion of the light-driven proton pump of Exiguobacterium sibiricum. Biochemistry 2012, 51 (29), 5748-62.

42. Imasheva, E. S.; Balashov, S. P.; Wang, J. M.; Dioumaev, A. K.; Lanyi, J. K., Selectivity of retinal photoisomerization in proteorhodopsin is controlled by aspartic acid 227. Biochemistry 2004, 43 (6), 1648-55.

43. Herz, J.; Verhoefen, M. K.; Weber, I.; Bamann, C.; Glaubitz, C.; Wachtveitl, J., Critical role of Asp227 in the photocycle of proteorhodopsin. Biochemistry 2012, 51 (28), 5589-600. 


\section{Future Directions: protein characterization using mass spectrometry and molecular dynamics simulations}

\section{Determine the solution structure of pHLIP in state I}

pHLIP is a negatively-charged, membrane-active peptide which potentially can be used to deliver drugs to the cellular environment ${ }^{1}$. Ongoing research is directed at determining the structure, interactions, and functional properties of this peptide. Researchers have divided the functional properties into three states ${ }^{2}$ : in state one the peptide is in solution and has no secondary structure; in state 2 the peptide is at the surface of a lipid bilayer and can bind to it under basic conditions while the structure is still random coil; and, finally, for the state 3 , by changing the $\mathrm{pH}$ to slightly acidic conditions which introduces positive charge to the acidic residues, pHLIP folds into an $\alpha$-helix and penetrates the bilayer ${ }^{3}$. The pHLIP structure in the solution phase (state I) is not completely understood. Identifying the helical region(s) of the pHLIP peptide at physiological and low $\mathrm{pH}$ is a crucial step to garner a better understanding of the peptide structure in state I. This could lead to more effective pHLIP targeting for tumor cells 4-6.

Different analytical tools such as circular dichroism (CD), NMR and fluorescence spectroscopy as well as other techniques like MD simulations have been utilized to characterize

pHLIP behavior in these states ${ }^{1,5,7-9}$. Online, solution hydrogen-deuterium exchange (HDX) can be used to identify the pHLIP structure when it is not interacting with lipids ${ }^{10}$. This approach can provide the amount of deuterium incorporation for each residue within the peptide. The amino acids that are more accessible (less involved in hydrogen bonding) to solution exchange will experience more deuterium uptake. That is, HDX can reveal the secondary structure of the peptide in solution at the amino acid residue level. This is accomplished with native MS and tandem mass spectrometry (MS/MS) employing electron transfer dissociation (ETD) ${ }^{11-12}$. 
Ion mobility spectrometry (IMS) coupled with gas-phase HDX (IMS-HDX) can also be used to determine the structural properties of the peptide ${ }^{13-15}$. Drift time measurements using IMS can provide information about the number of unique solution conformations that the peptide exhibits. By using ion mobility spectrometry- ion mobility spectrometry (IMS-IMS) we can select a specific conformer in the first IMS and introduce it to the second IMS in order to perform HDX. Recently, it has been shown that peptide ions in non-helical states have distinguished deuterium uptake compared to helical states using gas phase IMS and HDX ${ }^{16}$. Thus, a per-residue analysis such as that proposed here could reveal the location of helical portions of proteins/peptides. These techniques can be used in conjunction with the solution-phase HDX and MD simulations to reveal the location of helical turns in the pHLIP peptide as has been accomplished for model peptides ${ }^{17-22}$.

To confirm the structural changes when the $\mathrm{pH}$ is reduced from 7 to $2.5, \mathrm{CD}$ spectra were recorded for the wild type pHLIP peptide at the different $\mathrm{pH}$ values mentioned above. Fig. 5.1 shows that the helical propensity for the peptide increases from $\sim 2 \%$ to $10 \%$ when the $\mathrm{pH}$ is reduced from 7 to 2.5 . Finding the residues that are responsible for this helix formation is important. HDX coupled with an enzymatic digestion method can also be used to identify the helix portion ${ }^{14,23-24}$. Here, the ability of each fragment to uptake deuterium is calculated upon digestion of the peptide with the enzyme pepsin. 


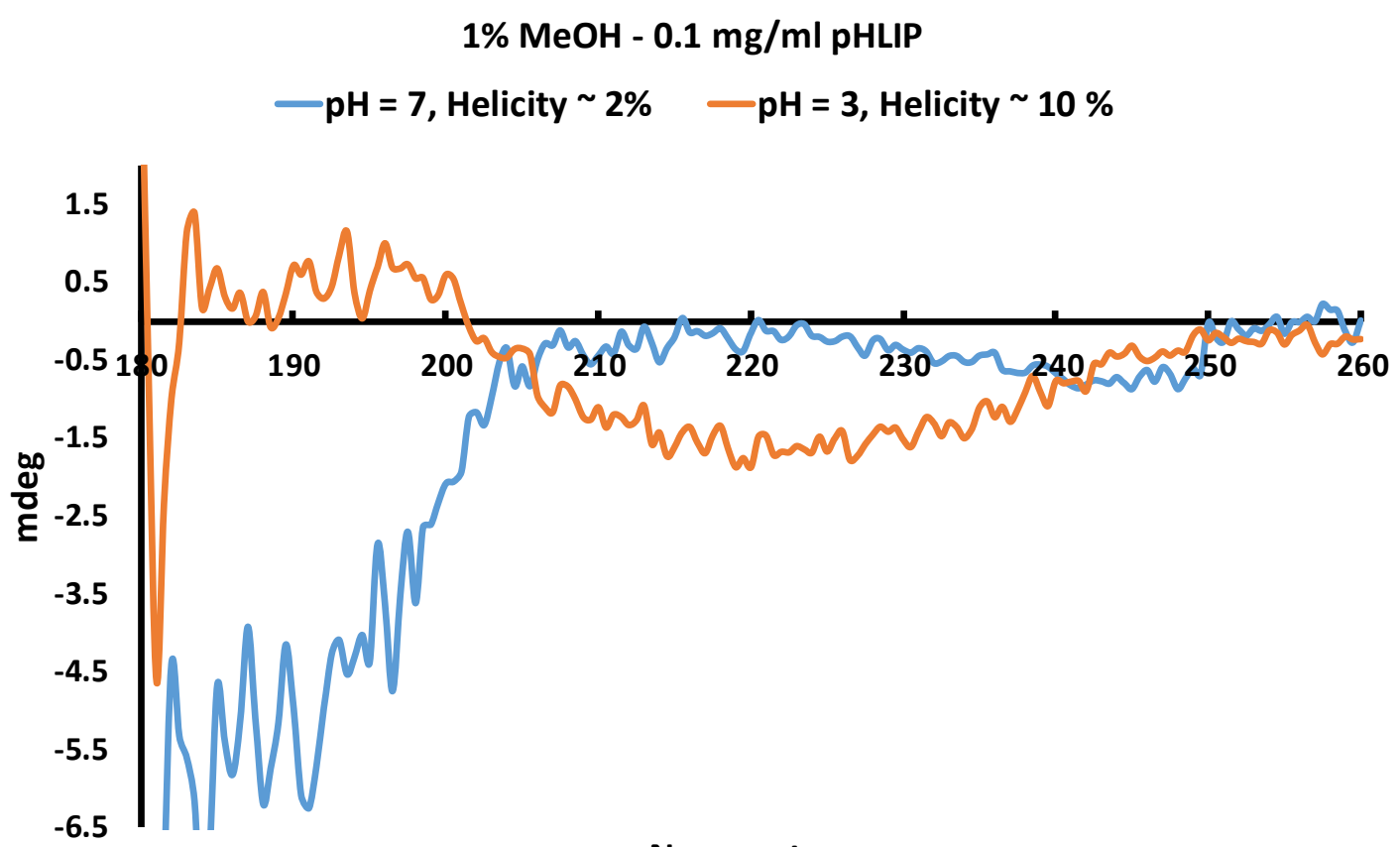

Nanometers

Figure 5. $1 \mathrm{CD}$ spectrum of pHLIP recorded at two different $\mathrm{pH}$ levels.

Figure 5.2 shows the setup that can be used in order to perform online, solution-phase hydrogen deuterium exchange ${ }^{10}$. In this setup the pHLIP peptide will interact with $\mathrm{D}_{2} \mathrm{O}$ for a select period of time based on solvent flow rates and capillary diameters. Subsequently the analyte solution will undergo online digestion using the enzyme pepsin. The digestion will also be conducted for a select period of time. The pHLIP: $\mathrm{D}_{2} \mathrm{O}$ ratio will be set to be 5:95. The flow rate for the pepsin is typically maintained at the flow rate for the peptide solution. Additionally, kinetics studies will be performed by changing the length of the reaction capillary and the flow rates of the solutions. For example, by changing the flow rate for the peptide solution to 2 $\mu \mathrm{L} / \mathrm{min}$ (Figure 5.2) and the flow rate for the $\mathrm{D}_{2} \mathrm{O}$ solution to $38 \mu \mathrm{L} / \mathrm{min}$, the HDX reaction time will increase to 10 seconds. 


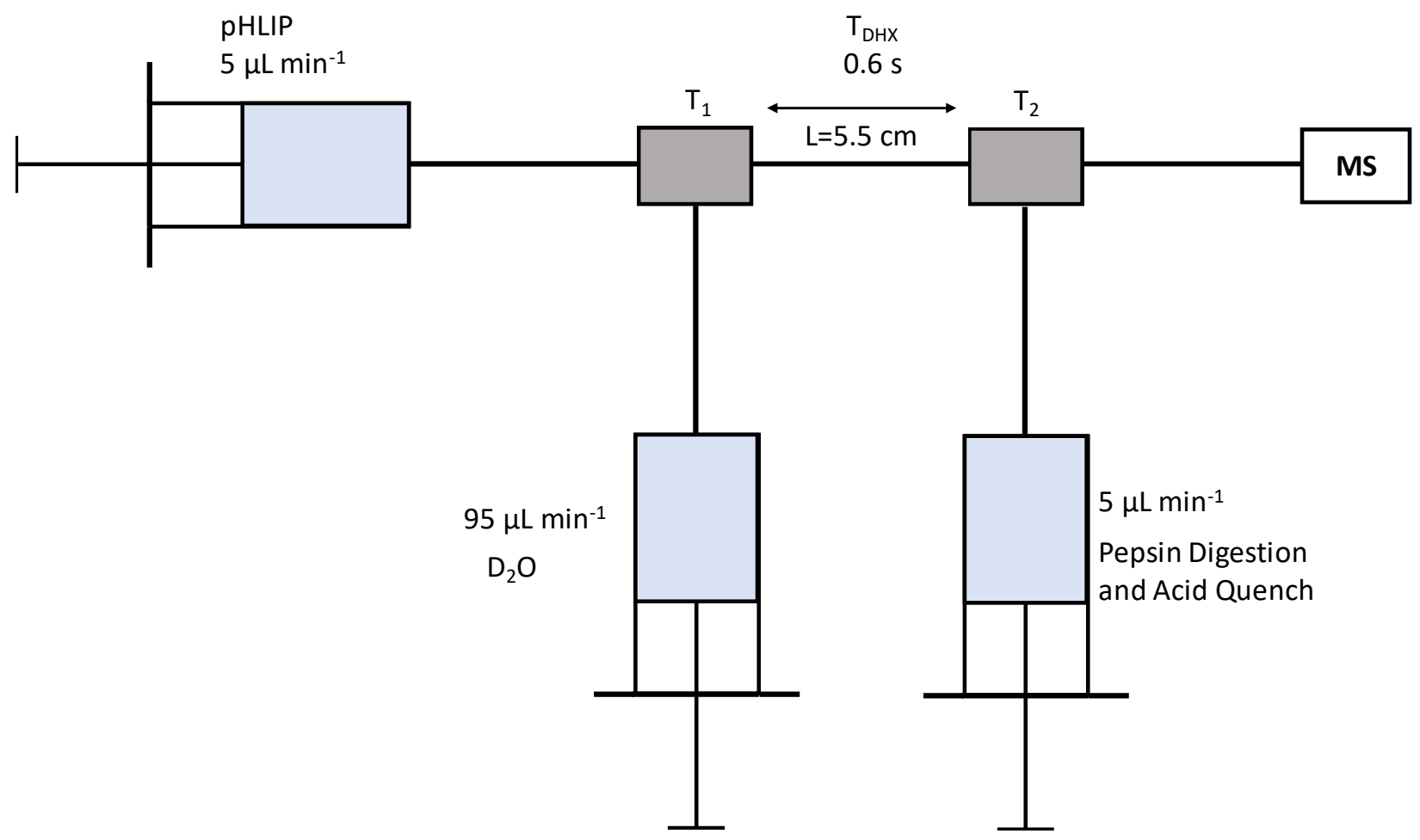

Figure 5. 2 Online hydrogen deuterium exchange apparatus. The peptide solution and water mixing occurs at T1 and HDX occurs along L. By changing this length, different exchange rates can be measured.

\section{Determine the effect of membrane composition on permeation of pHLIP in membrane}

For proteins that insert into the membrane, many contain mostly short peptide sequences that can penetrate the membrane and transport cargo or disrupt the function of the membrane upon insertion ${ }^{25-27}$. Studying these species and the method of insertion is important because it may permit, for example, the selection and optimization of drug delivery peptides based on their binding energies to the membrane. $\mathrm{pH}$ (low) insertion peptide (pHLIP) is a cell-active peptide. It is known that pHLIP inserts into the lipid bilayer membrane at $\mathrm{pH} \sim 6.1$ while a $\mathrm{pH}$ value around that associated with cancer cells is $\sim 6.8$. Therefore, there is a need to develop strategies to facilitate pHLIP insertion at higher $\mathrm{pH}$ values. A larger area per lipid might aid membrane-active 
peptide insertion. Differences in the lipid systems could play a role in the degree and type of peptide-lipid interactions. Membranes also have a capture and stabilization effect on the peptide. However, the insertion ability of this peptide requires the presence of both the membrane and a decreased $\mathrm{pH}$ value. The increase in area per lipid might reveal a threshold size for "turning on" peptide-lipid interaction (and thus insertion). That is, the peptide may be able to insert at a higher $\mathrm{pH}$ just by changing the area per lipid value of the membrane. The structural details of pHLIP (as a membrane-active peptide) including interactions with different lipid systems can be examined with native MS and MD simulations. Peptide-lipid bilayer interactions can be examined at neutral $\mathrm{pH}$ to study the effect of membrane composition. To obtain experimental information about the role of membrane composition on the lipid-binding ability of pHLIP, the insertion ability as a function of membrane (completely saturated as with DMPC to high area per lipid such as $4 \mathrm{ME} 16: 0 \mathrm{PC}$ ) can be investigated. It is expected that the peptide will insert more readily into the membrane with larger area per lipid. A better understanding of the conformational changes of the membrane-active peptide as the lipid system changes will provide a better understanding of how this peptide functions.

A recent NMR study shows that pHLIP in state II, when it is at the surface of a bilayer, exhibits an interaction with the POPC headgroups ${ }^{8}$. The study was performed at three different $\mathrm{pH}$ values. It was shown that, depending on the $\mathrm{pH}$, there is a distinct difference between the ability of pHLIP to get absorbed at the lipid surface. It was demonstrated that the peptide penetrates deeper into the membrane at $\mathrm{pH} \sim 6.4$. As a membrane with higher area per lipid value provides more spacing for the peptide to insert, it is interesting to study the interactions between the peptide with other PC lipids using native MS. Figure 5.3 represents the mass spectrum for the interaction between pHLIP and POPC lipid vesicle. 


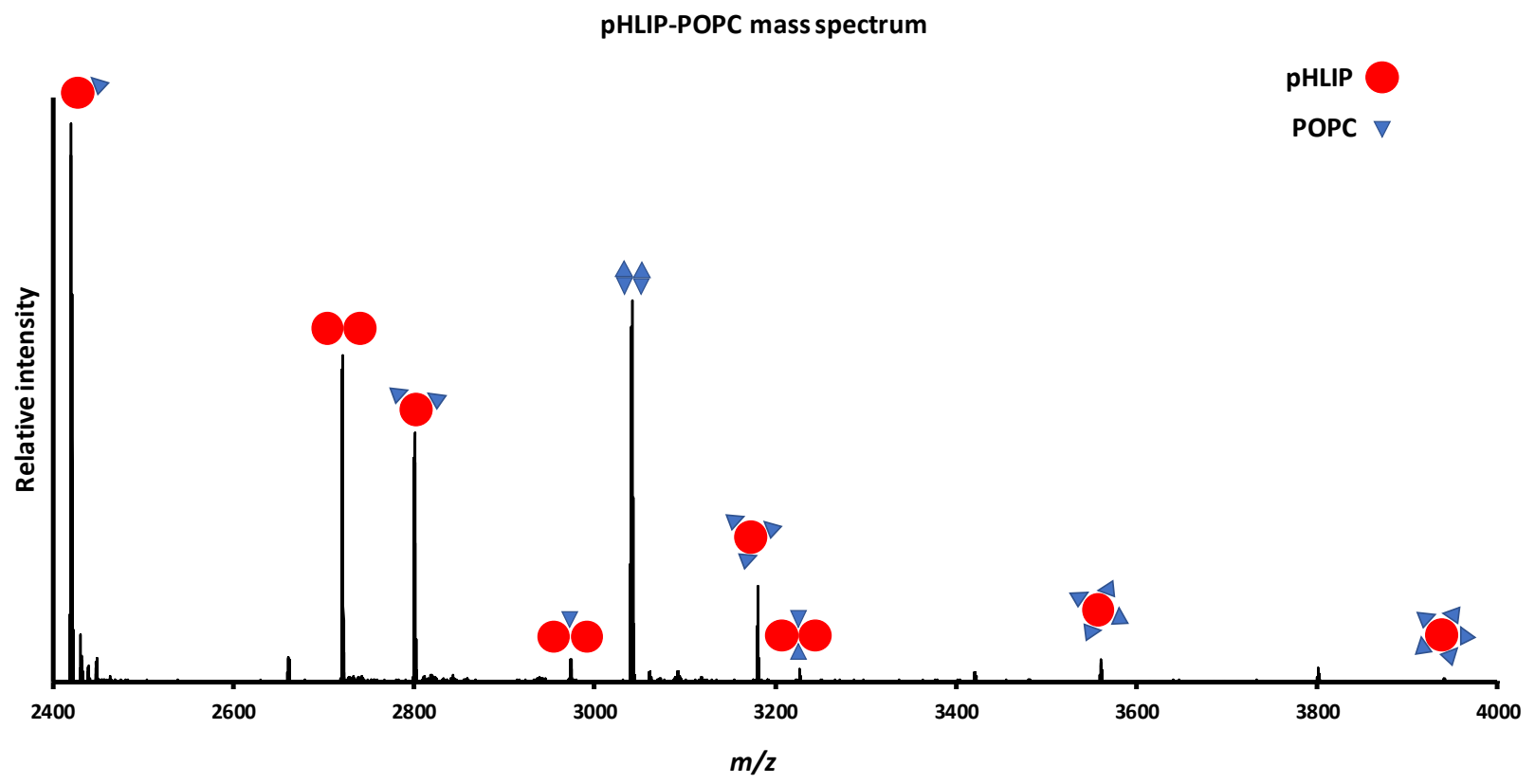

Figure 5. 3 The mass spectrum (ESI-MS) for a pHLIP-POPC system. Different monomeric and multimeric ions are labeled (see legend for details.

As mentioned above, because cancer cells exist in a slightly acidic medium, pHLIP can differentiate between a cancer cell and healthy cells ${ }^{2}$. Therefore, pHLIP insertion into a membrane thus makes it a good candidate for targeted therapy ${ }^{28}$. That said, initial peptide-lipid interactions can represent an essential step associated with a variety of membrane-active peptide functions or they can represent other serious disorders resulting from oligomerization of a non-penetrating peptide ${ }^{29-35}$. As such, many other physiological conditions are directly related to initial interactions between the peptide's residues and the hydrophilic head group or the hydrophobic tail of the membrane. Providing detailed knowledge of the peptide-lipid membrane interactions can ultimately result in the development of new treatment strategies for different diseases. 


\section{The strange case of leptin; an electrospray ionization study}

lonizing molecules is an obvious prerequisite step for mass spectrometry (MS) experiments ${ }^{36}$. Due to the softness of the method, the ability of providing multiply-charged ions, and the ease of coupling with analytical instruments, the most common technique to accomplish the ionization step in commercial MS devices is electrospray lonization (ESI) ${ }^{37}$. The importance of studying the mechanism of the ESI process is in intelligently designing better experiments using ESI-MS as well as for the construction of improved MS instrumentation. In ESI, a solution is infused through a needle that is biased relative to the mass spectrometer inlet. The applied electric field causes a destabilization of the liquid meniscus and the formation of a Tayler cone 36-39. This generates a stream that will disintegrate into micrometer-sized droplets with excess charge. These droplets will then undergo a series of solvent evaporation and fission events until the naked analyte ion is introduced into the mass spectrometer. The process of evaporationfission can happen several times up to a point such that the radius is small enough $(<10 \mathrm{~nm})$ for the molecule to leave the droplet ${ }^{37}$. It is possible to obtain different populations of ions having different numbers of protons attached and creating characteristic charge state distributions in the mass spectrum.

For small ions (low molecular weight such as sodium), the proposed mechanism is the ion evaporation model (IEM) ${ }^{40-41}$. In this mechanism small ions can eject from a droplet in the final stages of the ESI process. Conversely, for high molecular weight systems such as proteins, two mechanisms have been proposed including: the charged residue model (CRM) ${ }^{42-44}$ and the chain ejection model (CEM) ${ }^{36}$.

Notably, the charge state distribution (CSD) of proteins in the mass spectrum is significantly different for folded and unfolded proteins which suggests that the ionization process is different for these two states; CEM is suggested for proteins in their folded state and the CRM is for unfolded proteins ${ }^{45}$. In CEM hydrophobic and electrostatic forces drive the protein to the 
surface of the droplet and when the protein is expelled from the droplet, some charges at the surface of the droplet will be transferred to the protein ${ }^{45}$. In CRM, the evaporation continues until the solvent is completely evaporated and the remaining charge is transferred to the protein 44, 46. For proteins with a mixture of secondary structure and unstructured regions, the mechanism might be different. For example, leptin is a globular protein which is responsible for normalizing body weight ${ }^{47-49}$. A recently acquired ESI-MS spectrum for this protein is shown in Figure 5.4 (B). Here the protein was electrosprayed using native MS solvent conditions. Noticeably the spectrum consists of two regions: one region with low CSD centered at +8 and a second region with high CSD centered at +11 . For comparison purposes, a spectrum for the globular protein myoglobin conducted under the same conditions as those for the leptin protein was collected (Figure 5.4). For myoglobin, the overall number of separate charge states is reduced, and they are centered around +8 . In addition, the signal level for the leptin protein was about an order of magnitude larger than that of myoglobin. Taken together, this indicates that the ionization method could be very different for leptin; notably, as a compact globular protein, myoglobin may be expected to follow the traditional CRM process. Such a difference may resul;t from the unstructured region of the leptin protein which results in the bi-model CSD. Analyzing the crystal structure of leptin ${ }^{47}$ reveals that $35 \%$ of the residues comprise random coil regions which mostly range from Ser25 to Gly38 (14 residues) and Leu39 to Thr 50 (11 residues) and Ser95 to Leu107 (12 residues). This unstructured region comprises almost an entire face of the protein. The same analysis for myoglobin ${ }^{50}$ shows that only $20 \%$ of the residues are in the random coil regions and the longest stretch of random coil residues ranges from Phe43 to Thr 51 (9 residues).

To further investigate the electrospray ionization behavior of these two proteins we utilized molecular dynamics (MD) simulations. Most of the mechanistic studies for the last stages of the ESI process have been performed by MD simulations. Two water droplet systems 
with $\sim 4.5 \mathrm{~nm}$ radius and a protein in their center have been initially modeled. The droplet charge for each system is assigned to be lower than the Rayleigh limit. Figure 5.5 depicts the movements of each protein within its respective droplet. The MD simulations show that the leptin protein tends to present near the surface of the droplet while the Myoglobin remains closer to the droplet center. The snapshots of these movements show that the random coil regions of the leptin protein play an important role in driving the protein to the droplet surface. Clearly, repeated studies must be conducted to conclude that such behavior is affected by these regions and this is not just Brownian motion. Additionally, a longer simulation time is required to see the ejection of the protein from the droplet. Konermann and co-workers have already shown that for a completely denatured protein the ejection happens in $<10$ ns time frame ${ }^{45}$. A difference is the overall size of the water droplet. The larger size was selected to better track the interaction with the droplet surface over time. 
(A) Myoglobin, normalized level: 3.03E3
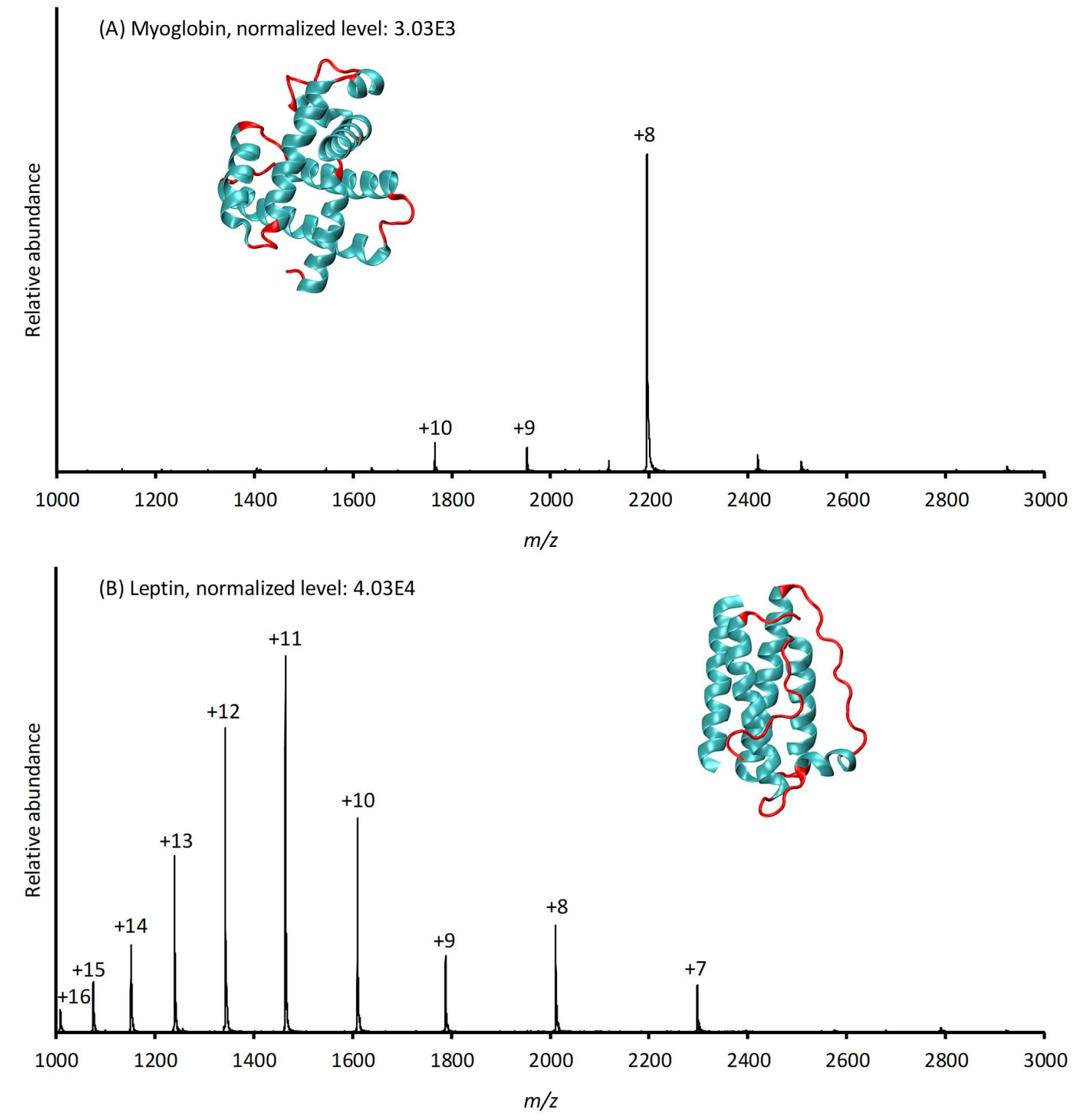

Figure 5. 4 Mass spectra for for A) Myoglobin and B) leptin generated by native MS. Here the proteins were electrosprayed from buffered (100 mM ammonium acetate) solutions. Charge states are labeled for each protein. Insets show the structures of the two different proteins. Note the long, unstructured region for the protein leptin which comprises an entire face of the protein. 


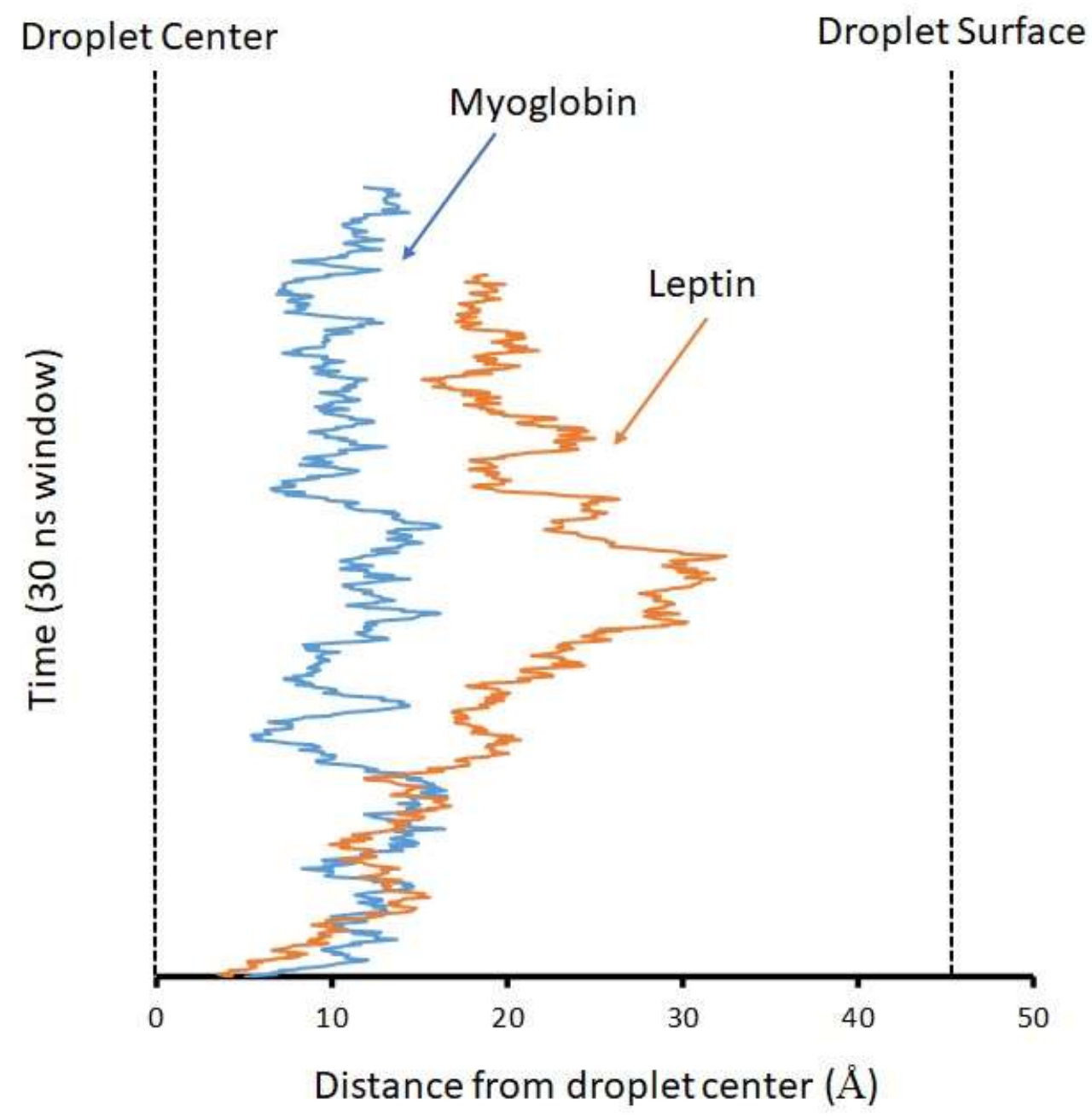

Figure 5. 5 The distance profile of the center of mass for myoglobin and leptin proteins to the water droplet center of mass. The distance to the droplet surface is provided. The two different traces are labeled for the respective proteins.

Modeling the CRM behavior of proteins will require a substantially a longer time ${ }^{39,51-52}$. Future studies should be conducted in which the droplet drying is allowed to proceed to the point of ion ejection or ion formation via CRM. From multiple analyses, it may be possible to deduce whether or not it will be possible to form leptin ions via the CEM process. Other experiments can be conducted in which proteins of varying degrees of unstructured region are examined to observe whether or not the CEM holds for those of higher unstructured state. To these studies, 
ion mobility spectrometry investigations can be added. For example, with IMS it will be possible to determine whether or not the protein ions exhibit a greater change in conformational size as evidenced by comparisons of ion cross sections with those calculated for the native state using the crystal structure coordinates. These same experiments can also be conducted for the similar proteins with long unstructured regions comprised of $>30 \%$ random coil residues.

\section{References}

1. Gupta, C.; Mertz, B., Protonation Enhances the Inherent Helix-Forming Propensity of pHLIP. ACS Omega 2017, 2 (11), 8536-8542.

2. Gupta, C.; Ren, Y.; Mertz, B., Cooperative Nonbonded Forces Control Membrane Binding of the pH-Low Insertion Peptide pHLIP. Biophys J 2018, 115 (12), 2403-2412.

3. Reshetnyak, Y. K.; Segala, M.; Andreev, O. A.; Engelman, D. M., A monomeric membrane peptide that lives in three worlds: in solution, attached to, and inserted across lipid bilayers. Biophys $J$ 2007, 93 (7), 2363-72.

4. $\quad$ Rinaldi, F.; Hanieh, P. N.; Del Favero, E.; Rondelli, V.; Brocca, P.; Pereira, M. C.; Andreev, O. A.; Reshetnyak, Y. K.; Marianecci, C.; Carafa, M., Decoration of Nanovesicles with pH (Low) Insertion Peptide (pHLIP) for Targeted Delivery. Nanoscale Res Lett 2018, 13 (1), 391.

5. Wyatt, L. C.; Moshnikova, A.; Crawford, T.; Engelman, D. M.; Andreev, O. A.; Reshetnyak, Y. K., Peptides of pHLIP family for targeted intracellular and extracellular delivery of cargo molecules to tumors. Proc Natl Acad Sci U S A 2018, 115 (12), E2811-E2818.

6. Vasquez-Montes, V.; Gerhart, J.; Thevenin, D.; Ladokhin, A. S., Divalent Cations and Lipid Composition Modulate Membrane Insertion and Cancer-Targeting Action of pHLIP. J Mol Biol 2019, 431 (24), 5004-5018.

7. Hanz, S. Z.; Shu, N. S.; Qian, J.; Christman, N.; Kranz, P.; An, M.; Grewer, C.; Qiang, W., Protonation-Driven Membrane Insertion of a pH-Low Insertion Peptide. Angew Chem Int Ed Engl 2016, 55 (40), 12376-81.

8. Shu, N. S.; Chung, M. S.; Yao, L.; An, M.; Qiang, W., Residue-specific structures and membrane locations of pH-low insertion peptide by solid-state nuclear magnetic resonance. Nat Commun 2015, 6, 7787.

9. Segala, J.; Engelman, D. M.; Reshetnyak, Y. K.; Andreev, O. A., Accurate analysis of tumor margins using a fluorescent pH Low Insertion Peptide (pHLIP). Int J Mol Sci 2009, 10 (8), 3478-87.

10. Donohoe, G. C.; Arndt, J. R.; Valentine, S. J., Online deuterium hydrogen exchange and protein digestion coupled with ion mobility spectrometry and tandem mass spectrometry. Anal Chem 2015, 87 (10), 5247-54.

11. Huang, R. Y.; Garai, K.; Frieden, C.; Gross, M. L., Hydrogen/deuterium exchange and electrontransfer dissociation mass spectrometry determine the interface and dynamics of apolipoprotein $\mathrm{E}$ oligomerization. Biochemistry 2011, 50 (43), 9273-82.

12. Syka, J. E. P.; Coon, J. J.; Schroeder, M. J.; Shabanowitz, J.; Hunt, D. F., Peptide and protein sequence analysis by electron transfer dissociation mass spectrometry. Proc. Natl. Acad. Sci. U. S. A. 2004, 101 (26), 9528-9533. 
13. Mistarz, U. H.; Brown, J. M.; Haselmann, K. F.; Rand, K. D., Simple Setup for Gas-Phase H/D Exchange Mass Spectrometry Coupled to Electron Transfer Dissociation and Ion Mobility for Analysis of Polypeptide Structure on a Liquid Chromatographic Time Scale. Anal. Chem. 2014, 86 (23), 11868-11876. 14. Rand, K. D.; Pringle, S. D.; Morris, M.; Brown, J. M., Site-Specific Analysis of Gas-Phase Hydrogen/Deuterium Exchange of Peptides and Proteins by Electron Transfer Dissociation. Anal. Chem. 2012, 84 (4), 1931-1940.

15. Zehl, M.; Rand, K. D.; Jensen, O. N.; Jorgensen, T. J., Electron transfer dissociation facilitates the measurement of deuterium incorporation into selectively labeled peptides with single residue resolution. J Am Chem Soc 2008, 130 (51), 17453-9.

16. Majuta, S. N.; Maleki, H.; Kiani Karanji, A.; Attanyake, K.; Loch, E.; Valentine, S. J., Magnifying ion mobility spectrometry-mass spectrometry measurements for biomolecular structure studies. Curr Opin Chem Biol 2018, 42, 101-110.

17. Khakinejad, M.; Kondalaji, S. G.; Maleki, H.; Arndt, J. R.; Donohoe, G. C.; Valentine, S. J., Combining ion mobility spectrometry with hydrogen-deuterium exchange and top-down MS for peptide ion structure analysis. J Am Soc Mass Spectrom 2014, 25 (12), 2103-15.

18. Donohoe, G. C.; Khakinejad, M.; Valentine, S. J., lon mobility spectrometry-hydrogen deuterium exchange mass spectrometry of anions: part 1. Peptides to proteins. J Am Soc Mass Spectrom 2015, 26 (4), 564-76.

19. Khakinejad, M.; Kondalaji, S. G.; Tafreshian, A.; Valentine, S. J., Gas-Phase Hydrogen-Deuterium Exchange Labeling of Select Peptide Ion Conformer Types: a Per-Residue Kinetics Analysis. J Am Soc Mass Spectrom 2015.

20. Khakinejad, M.; Ghassabi Kondalaji, S.; Tafreshian, A.; Valentine, S. J., Comprehensive Gas-Phase Peptide Ion Structure Studies Using Ion Mobility Techniques: Part 2. Gas-Phase Hydrogen/Deuterium Exchange for Ion Population Estimation. J Am Soc Mass Spectrom 2017, 28 (5), 960-970.

21. Ghassabi Kondalaji, S.; Khakinejad, M.; Tafreshian, A.; S, J. V., Comprehensive Peptide Ion Structure Studies Using Ion Mobility Techniques: Part 1. An Advanced Protocol for Molecular Dynamics Simulations and Collision Cross-Section Calculation. J Am Soc Mass Spectrom 2017, 28 (5), 947-959.

22. Karanji, A. K.; Khakinejad, M.; Kondalaji, S. G.; Majuta, S. N.; Attanayake, K.; Valentine, S. J., Comparison of Peptide Ion Conformers Arising from Non-Helical and Helical Peptides Using Ion Mobility Spectrometry and Gas-Phase Hydrogen/Deuterium Exchange. J Am Soc Mass Spectrom 2018, 29 (12), 2402-2412.

23. Karch, K. R.; Coradin, M.; Zandarashvili, L.; Kan, Z. Y.; Gerace, M.; Englander, S. W.; Black, B. E.; Garcia, B. A., Hydrogen-Deuterium Exchange Coupled to Top- and Middle-Down Mass Spectrometry Reveals Histone Tail Dynamics before and after Nucleosome Assembly. Structure 2018, 26 (12), 16511663 e3.

24. Rand, K. D.; Zehl, M.; Jensen, O. N.; Jorgensen, T. J., Loss of ammonia during electron-transfer dissociation of deuterated peptides as an inherent gauge of gas-phase hydrogen scrambling. Anal Chem 2010, 82 (23), 9755-62.

25. Plank, C.; Zauner, W.; Wagner, E., Application of membrane-active peptides for drug and gene delivery across cellular membranes. Advanced Drug Delivery Reviews 1998, 34 (1), 21-35.

26. Sani, M. A.; Separovic, F., How Membrane-Active Peptides Get into Lipid Membranes. Acc Chem Res 2016, 49 (6), 1130-8.

27. Wagner, E., Application of membrane-active peptides for nonviral gene delivery. Advanced Drug Delivery Reviews 1999, 38 (3), 279-289.

28. An, M.; Wijesinghe, D.; Andreev, O. A.; Reshetnyak, Y. K.; Engelman, D. M., pH-(low)-insertionpeptide (pHLIP) translocation of membrane impermeable phalloidin toxin inhibits cancer cell proliferation. Proc Natl Acad Sci U S A 2010, 107 (47), 20246-50. 
29. Ambroggio, E. E.; Separovic, F.; Bowie, J.; Fidelio, G. D., Surface behaviour and peptide-lipid interactions of the antibiotic peptides, Maculatin and Citropin. Biochim Biophys Acta 2004, 1664 (1), 317.

30. Batenburg, A. M.; de Kruijff, B., Modulation of membrane surface curvature by peptide-lipid interactions. Biosci Rep 1988, 8 (4), 299-307.

31. Bonev, B.; Watts, A.; Bokvist, M.; Gröbner, G., Electrostatic peptide-lipid interactions of amyloid- $\beta$ peptide and pentalysine with membrane surfaces monitored by 31P MAS NMR. Physical Chemistry Chemical Physics 2001, 3 (14), 2904-2910.

32. Bruni, R.; Taeusch, H. W.; Waring, A. J., Surfactant protein B: lipid interactions of synthetic peptides representing the amino-terminal amphipathic domain. Proc Natl Acad Sci U S A 1991, 88 (16), 7451-5.

33. Maler, L., Solution NMR studies of peptide-lipid interactions in model membranes. Mol Membr Biol 2012, 29 (5), 155-76.

34. Matsuzaki, K., Why and how are peptide-lipid interactions utilized for self-defense? Magainins and tachyplesins as archetypes. Biochimica et Biophysica Acta (BBA) - Biomembranes 1999, 1462 (1-2), 1-10.

35. Sanderson, J. M., Peptide-lipid interactions: insights and perspectives. Org Biomol Chem 2005, 3 (2), 201-12.

36. Konermann, L.; Ahadi, E.; Rodriguez, A. D.; Vahidi, S., Unraveling the mechanism of electrospray ionization. Anal Chem 2013, 85 (1), 2-9.

37. Kebarle, P.; Verkerk, U. H., Electrospray: from ions in solution to ions in the gas phase, what we know now. Mass Spectrom Rev 2009, 28 (6), 898-917.

38. Fenn, J. B.; Mann, M.; Meng, C. K.; Wong, S. F.; Whitehouse, C. M., Electrospray ionization for mass spectrometry of large biomolecules. Science 1989, 246 (4926), 64-71.

39. McAllister, R. G.; Metwally, H.; Sun, Y.; Konermann, L., Release of Native-like Gaseous Proteins from Electrospray Droplets via the Charged Residue Mechanism: Insights from Molecular Dynamics Simulations. J Am Chem Soc 2015, 137 (39), 12667-76.

40. Labowsky, M.; Fenn, J. B.; Fernandez de la Mora, J., A continuum model for ion evaporation from a drop: effect of curvature and charge on ion solvation energy. Analytica Chimica Acta 2000, 406 (1), 105-118.

41. Wang, G.; Cole, R. B., Charged residue versus ion evaporation for formation of alkali metal halide cluster ions in ESI. Analytica Chimica Acta 2000, 406 (1), 53-65.

42. Li, J.; Santambrogio, C.; Brocca, S.; Rossetti, G.; Carloni, P.; Grandori, R., Conformational effects in protein electrospray-ionization mass spectrometry. Mass Spectrom Rev 2016, 35 (1), 111-22.

43. Fernandez de la Mora, J., Electrospray ionization of large multiply charged species proceeds via Dole's charged residue mechanism. Analytica Chimica Acta 2000, 406 (1), 93-104.

44. Hogan, C. J., Jr.; Carroll, J. A.; Rohrs, H. W.; Biswas, P.; Gross, M. L., Combined charged residuefield emission model of macromolecular electrospray ionization. Anal Chem 2009, 81 (1), 369-77.

45. Metwally, H.; Duez, Q.; Konermann, L., Chain Ejection Model for Electrospray Ionization of Unfolded Proteins: Evidence from Atomistic Simulations and Ion Mobility Spectrometry. Anal Chem 2018, 90 (16), 10069-10077.

46. Felitsyn, N.; Peschke, M.; Kebarle, P., Origin and number of charges observed on multiplyprotonated native proteins produced by ESI. International Journal of Mass Spectrometry 2002, 219 (1), 39-62.

47. Zhang, F.; Basinski, M. B.; Beals, J. M.; Briggs, S. L.; Churgay, L. M.; Clawson, D. K.; DiMarchi, R. D.; Furman, T. C.; Hale, J. E.; Hsiung, H. M.; Schoner, B. E.; Smith, D. P.; Zhang, X. Y.; Wery, J. P.; Schevitz, R. W., Crystal structure of the obese protein leptin-E100. Nature 1997, 387 (6629), 206-9. 
48. Prokop, J. W.; Duff, R. J.; Ball, H. C.; Copeland, D. L.; Londraville, R. L., Leptin and leptin receptor: analysis of a structure to function relationship in interaction and evolution from humans to fish. Peptides 2012, 38 (2), 326-36.

49. Munzberg, H.; Morrison, C. D., Structure, production and signaling of leptin. Metabolism 2015, 64 (1), 13-23.

50. Maurus, R.; Overall, C. M.; Bogumil, R.; Luo, Y.; Mauk, A. G.; Smith, M.; Brayer, G. D., A myoglobin variant with a polar substitution in a conserved hydrophobic cluster in the heme binding pocket. Biochimica et Biophysica Acta (BBA) - Protein Structure and Molecular Enzymology 1997, 1341 (1), 1-13.

51. Ghassabi Kondalaji, S. K., M.; Valentine, S. J., Comprehensive Peptide Ion Structure Studies Using Ion Mobility Techniques: Part 3. Relating Solution-phase to Gas-phase Structures. Journal of the American Society for Mass Spectrometry (accepted) 2018.

52. Kim, D.; Wagner, N.; Wooding, K.; Clemmer, D. E.; Russell, D. H., lons from Solution to the Gas Phase: A Molecular Dynamics Simulation of the Structural Evolution of Substance P during Desolvation of Charged Nanodroplets Generated by Electrospray lonization. J Am Chem Soc 2017, 139 (8), 29812988.

53. Kucerka, N.; Nagle, J. F.; Sachs, J. N.; Feller, S. E.; Pencer, J.; Jackson, A.; Katsaras, J., Lipid bilayer structure determined by the simultaneous analysis of neutron and X-ray scattering data. Biophys J 2008, 95 (5), 2356-67.

54. Kucerka, N.; Nieh, M. P.; Katsaras, J., Fluid phase lipid areas and bilayer thicknesses of commonly used phosphatidylcholines as a function of temperature. Biochim Biophys Acta 2011, 1808 (11), 2761-71. 55. Jambeck, J. P.; Lyubartsev, A. P., An Extension and Further Validation of an All-Atomistic Force Field for Biological Membranes. J Chem Theory Comput 2012, 8 (8), 2938-48. 


\section{Appendix A}

\section{Figure Captions}

Figure S2. 1. CD spectroscopy of both model peptides PAAAAKAAAAKAAAAKAAAAK and PSSSSKSSSSKSSSSKSSSSK. The alanine sequence is showing a high-degree of helical character as indicated by the negative bands at $\sim 207$ and $223 \mathrm{~nm}$ as well as the positive band at $\sim 190 \mathrm{~nm}$. For the serine sequence peptide, the helicity is missing and a broad band at lower wavelengths is indicative of more random coil character.

Figure S2. 2. Conformational space sampling of 40-ps and 1200-ps SA for each conformer arrangement. The structures with the collision cross section within $\pm 2 \%$ are in red dash line section.

Figure S2. 3. An example of very compact ion conformer type with charge configuration of K6_K11_K21 and theoretical CCS value of $348 \AA^{2}$ Calculated by Mobcal software. This structure has a significant charge solvation of the charge sites by the serine residues.

Figure S2. 4. Schematic of S4 and S5 is explaining the increase in the predicted deuterium content for the conformer with high accessibility at the terminal peptide portions rather than the interior residues. These residues are close to the carbonyl groups showed by stars.

Figure S2. 5. A) One structure with a wrong CCS value but showing a high D incorporation value for S20. Hydroxyl and amine groups are bending back to the structure while for S18 these functional groups are facing outside. B) Visualizing the same structure on another view is showing that S2 is bending inside the structure and S4 is bending outside.

\section{Table Captions}

Table S2. 1. Deuterium uptake values calculation for serine residues in the model peptide. 


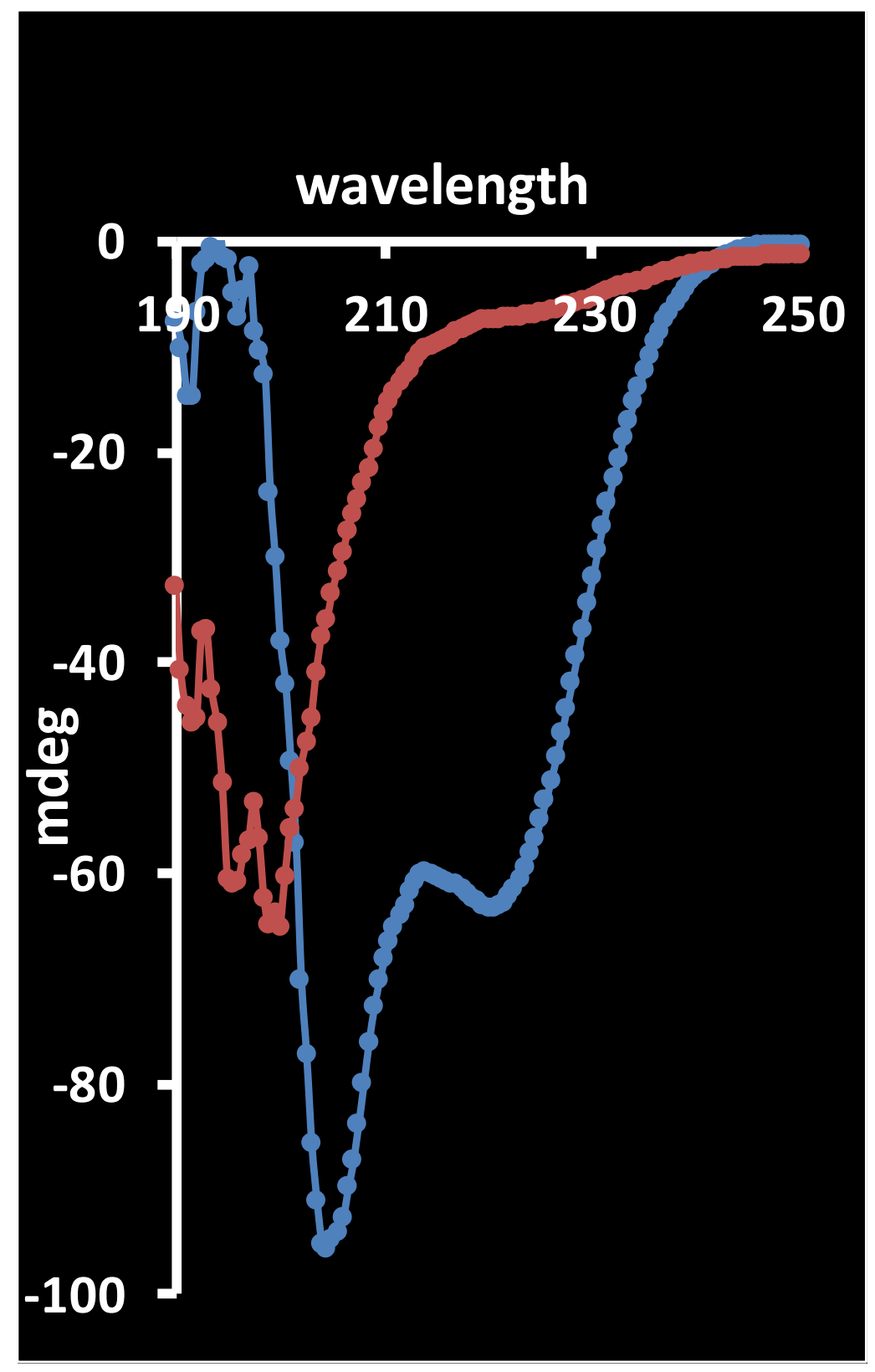

Figure S2. 1 CD spectroscopy of both model peptides PAAAAKAAAAKAAAAKAAAAK and PSSSSKSSSSKSSSSKSSSSK. 


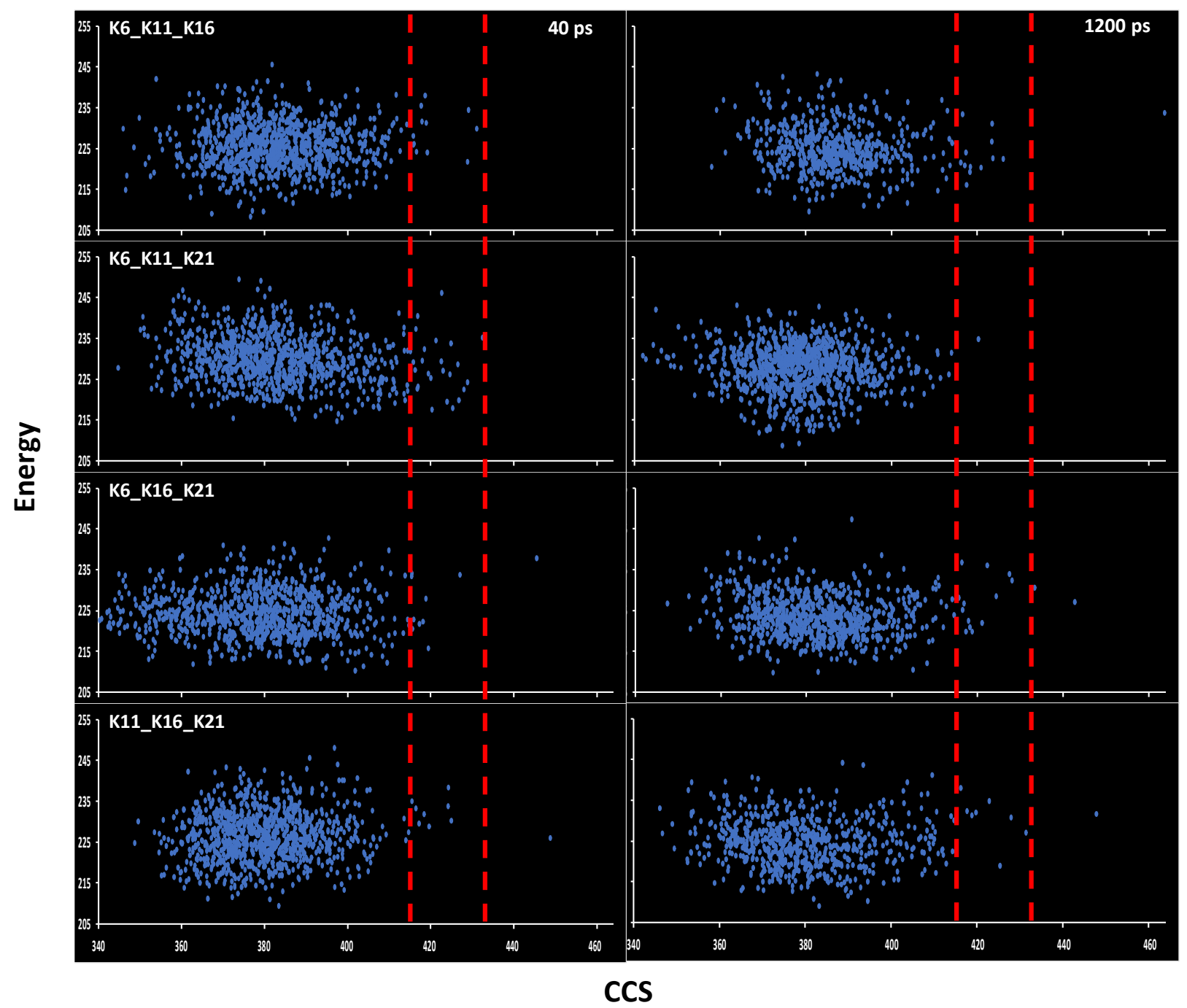

Figure S2. 2 Conformational space sampling of 40-ps and 1200-ps SA for each conformer arrangement. 


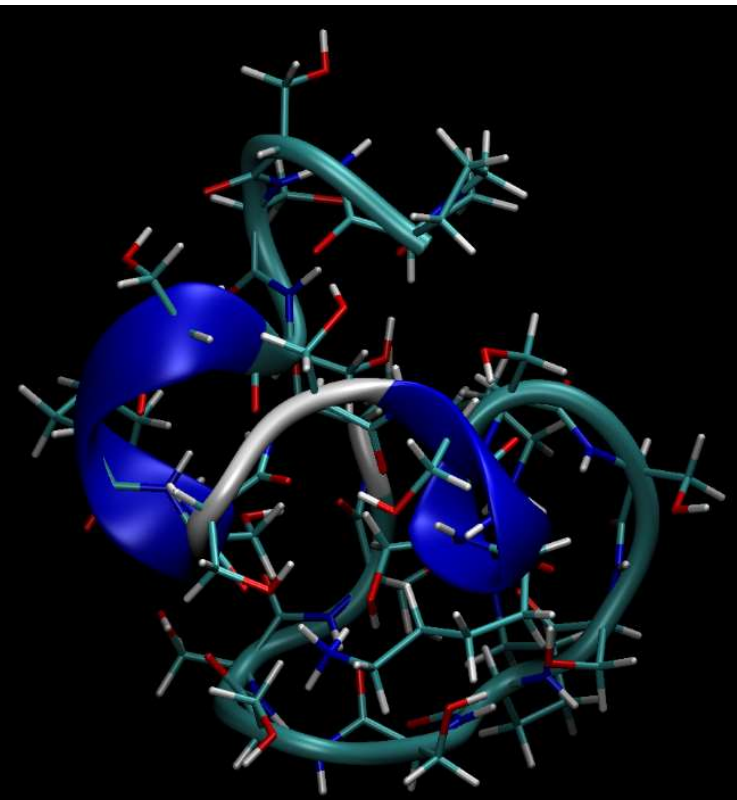

Figure S2. 3 An example of very compact ion conformer type with charge configuration of K6_K11_K21 and theoretical CCS value of 348 A2 Calculated by Mobcal software.

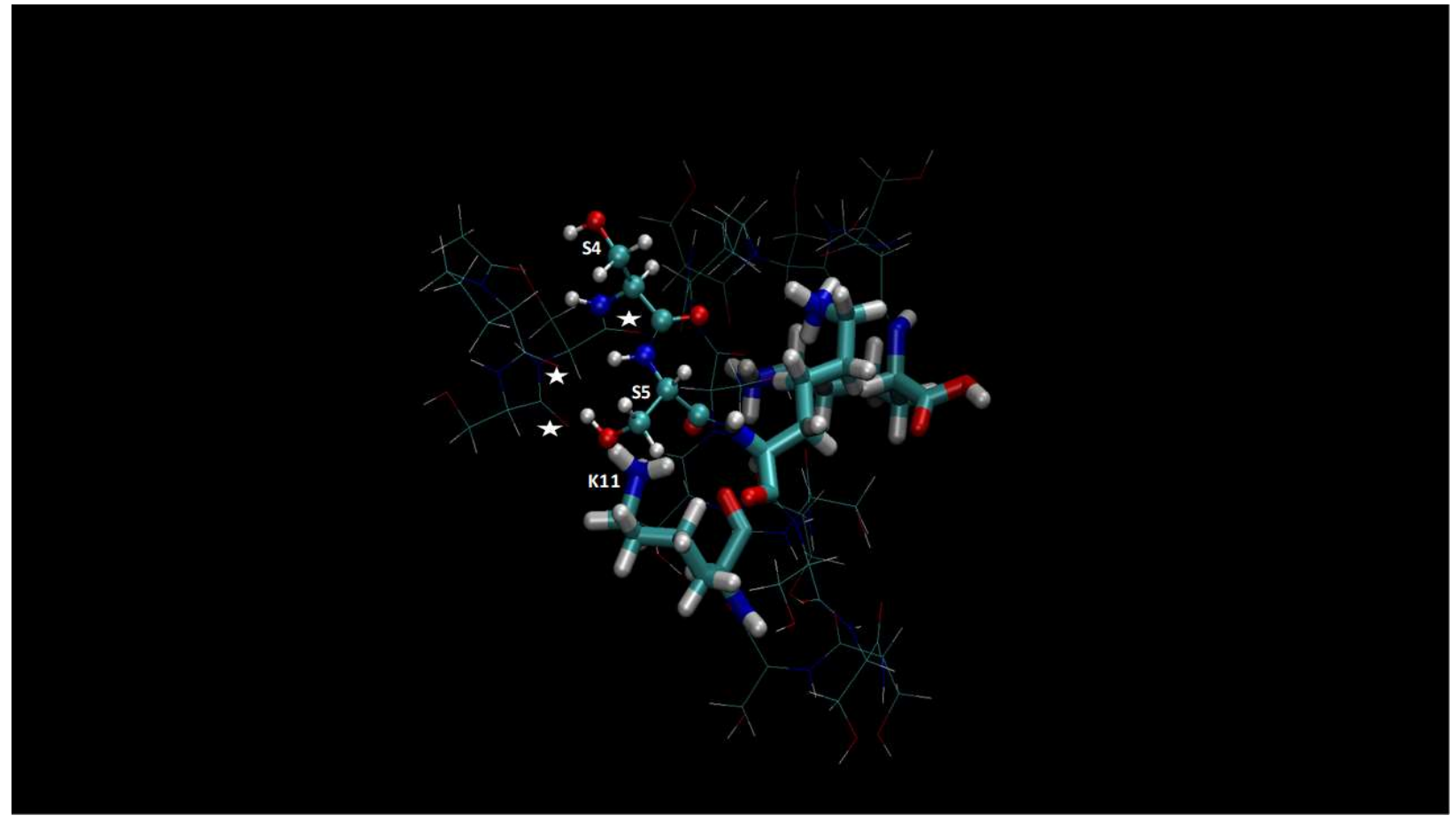

Figure S2. 4 The increase in the predicted deuterium content for the conformer with high accessibility at the terminal peptide portions rather than the interior residues. 
A

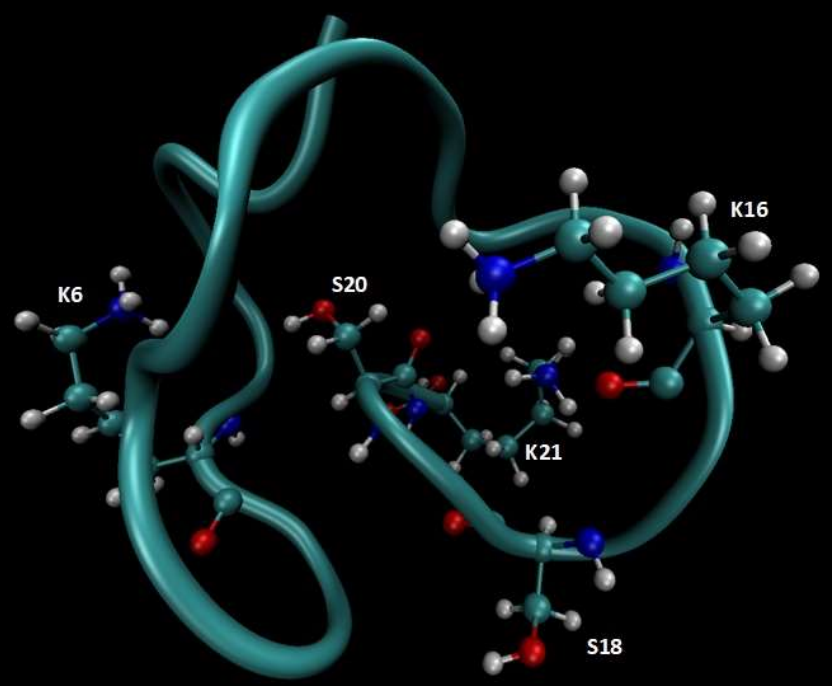

B

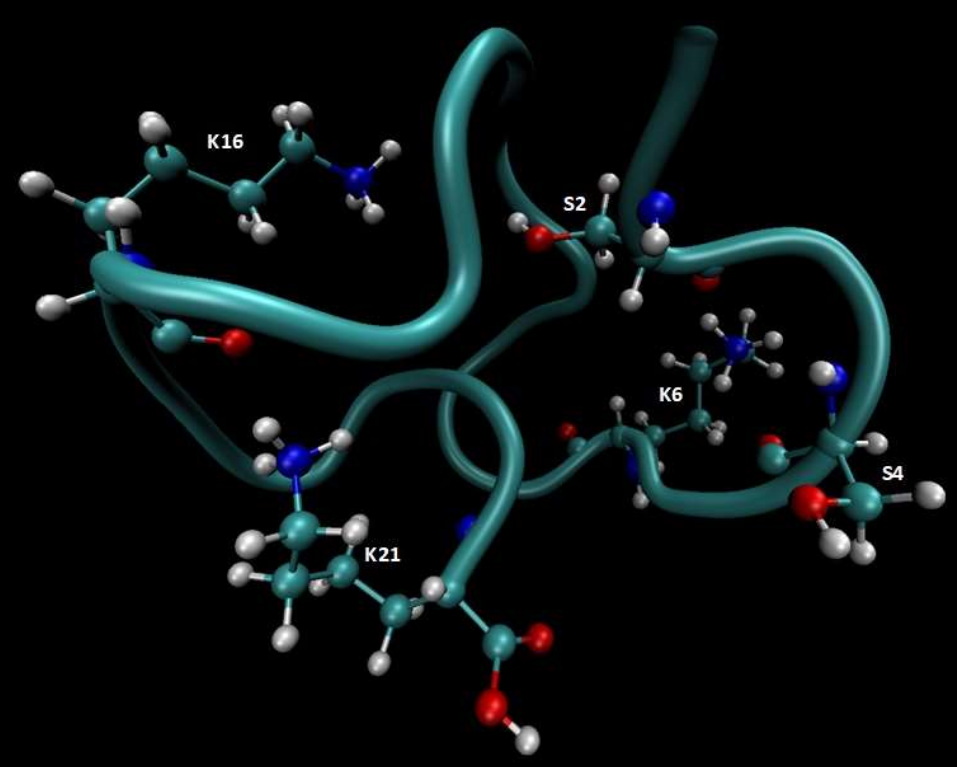

Figure S2. 5 An example of a structure with a wrong CCS value but showing a high D incorporation value for S20. 


\begin{tabular}{|c|c|c|c|c|}
\hline Residue Number & Fragments & $\mathrm{m} / \mathbf{z}^{\mathrm{a}}$ & $\mathrm{m} / \mathbf{z} \mathrm{D}_{2} \mathrm{O}$ & D uptake ${ }^{b}$ \\
\hline S2 & $z_{19}$ & 1821.88 & 1839.86 & 0.79 \\
\hline S3 & $\mathbf{z}_{18}$ & 1734.80 & 1752.52 & 0.26 \\
\hline S4 & $\mathbf{z}_{17}$ & 1647.72 & 1665.13 & 0.31 \\
\hline S5 & $\mathbf{z}_{16}$ & 1560.64 & 1577.47 & 0.58 \\
\hline s7 & $\mathbf{z}_{14}$ & 1345.39 & 1357.91 & 0.58 \\
\hline S8 & $z_{13}$ & 1258.31 & 1270.36 & 0.47 \\
\hline s9 & $z_{12}$ & 1171.23 & 1182.63 & 0.65 \\
\hline $\mathbf{S 1 0}$ & $z_{11}$ & 1084.15 & 1094.79 & 0.76 \\
\hline $\mathrm{S} 12$ & $\mathbf{Z}_{9}$ & 868.90 & 876.53 & 0.55 \\
\hline $\mathbf{s 1 3}$ & $\mathbf{z}_{8}$ & 781.82 & 788.94 & 0.51 \\
\hline S15 & $\mathbf{z}_{6}$ & 607.66 & 613.65 & 0.76 \\
\hline s7 & $\mathbf{C}_{7}$ & 720.76 & 726.59 & 0.75 \\
\hline S8 & $\mathrm{C}_{8}$ & 807.84 & 814.11 & 0.44 \\
\hline s9 & $C_{9}$ & 894.91 & 901.83 & 0.64 \\
\hline $\mathbf{S 1 0}$ & $c_{10}$ & 981.99 & 989.67 & 0.76 \\
\hline $\mathrm{S} 12$ & $\mathbf{c}_{12}$ & 1197.25 & 1207.91 & 0.56 \\
\hline $\mathrm{s} 13$ & $c_{13}$ & 1284.33 & 1295.47 & 0.48 \\
\hline $\mathrm{S} 14$ & $c_{14}$ & 1371.40 & 1383.14 & 0.59 \\
\hline S15 & $c_{15}$ & 1458.48 & 1470.92 & 0.70 \\
\hline S17 & $c_{17}$ & 1673.74 & 1689.80 & 0.60 \\
\hline S18 & $\mathrm{C}_{18}$ & 1760.82 & 1777.09 & 0.21 \\
\hline S19 & $c_{19}$ & 1847.89 & 1864.65 & 0.48 \\
\hline $\mathrm{S} 20$ & $c_{20}$ & 1934.97 & 1952.82 & 1.09 \\
\hline s2 & $z_{19}$ & 1821.88 & 1839.82 & 0.95 \\
\hline s3 & $z_{18}$ & 1734.80 & 1752.50 & 0.24 \\
\hline S4 & $\mathbf{z}_{17}$ & 1647.72 & 1665.16 & 0.26 \\
\hline S5 & $z_{16}$ & 1560.64 & 1577.51 & 0.57 \\
\hline S7 & $z_{14}$ & 1345.39 & 1358.06 & 0.78 \\
\hline S8 & $z_{13}$ & 1258.31 & 1270.42 & 0.56 \\
\hline s9 & $z_{12}$ & 1171.23 & 1182.71 & 0.63 \\
\hline $\mathbf{S 1 0}$ & $z_{11}$ & 1084.15 & 1094.84 & 0.79 \\
\hline S12 & $\mathbf{Z}_{9}$ & 868.90 & 876.53 & 0.58 \\
\hline $\mathrm{S} 13$ & $\mathbf{Z}_{8}$ & 781.82 & 789.00 & 0.45 \\
\hline S15 & $z_{6}$ & 607.66 & 613.62 & 0.81 \\
\hline s7 & $C_{7}$ & 720.76 & 726.40 & 0.67 \\
\hline S8 & $\mathrm{C}_{8}$ & 807.84 & 813.97 & 0.49 \\
\hline s9 & $C_{9}$ & 894.91 & 901.62 & 0.57 \\
\hline$S 10$ & $c_{10}$ & 981.99 & 989.59 & 0.89 \\
\hline $\mathrm{S} 12$ & $c_{12}$ & 1197.25 & 1208.01 & 0.67 \\
\hline $\mathrm{s} 13$ & $c_{13}$ & 1284.33 & 1295.54 & 0.45 \\
\hline $\mathrm{S} 14$ & $c_{14}$ & 1371.40 & 1383.10 & 0.48 \\
\hline S15 & $C_{15}$ & 1458.48 & 1470.88 & 0.70 \\
\hline $\mathrm{S} 17$ & $C_{17}$ & 1673.74 & 1689.94 & 0.69 \\
\hline
\end{tabular}




\begin{tabular}{|c|c|c|c|c|}
\hline Residue Number & Fragments & $\mathrm{m} / \mathbf{z}^{\mathrm{a}}$ & $\mathrm{m} / \mathbf{z} \mathrm{D}_{2} \mathrm{O}$ & D uptake ${ }^{b}$ \\
\hline S18 & $\mathrm{C}_{18}$ & 1760.82 & 1777.26 & 0.24 \\
\hline S19 & $C_{19}$ & 1847.89 & 1864.83 & 0.49 \\
\hline S20 & $\mathrm{C}_{20}$ & 1934.97 & 1952.80 & 0.89 \\
\hline S2 & $\mathbf{z}_{19}$ & 1821.88 & 1838.79 & 0.82 \\
\hline S3 & $\mathbf{z}_{18}$ & 1734.80 & 1751.25 & 0.46 \\
\hline S4 & $z_{17}$ & 1647.72 & 1663.77 & 0.40 \\
\hline S5 & $\mathbf{z}_{16}$ & 1560.64 & 1576.02 & 0.67 \\
\hline S7 & $z_{14}$ & 1345.39 & 1356.59 & 0.71 \\
\hline S8 & $\mathbf{z}_{13}$ & 1258.31 & 1269.03 & 0.48 \\
\hline s9 & $z_{12}$ & 1171.23 & 1181.28 & 0.67 \\
\hline S10 & $z_{11}$ & 1084.15 & 1093.51 & 0.69 \\
\hline S12 & $\mathbf{Z}_{9}$ & 868.90 & 875.23 & 0.56 \\
\hline S13 & $\mathbf{z}_{8}$ & 781.82 & 787.71 & 0.44 \\
\hline S15 & $\mathbf{z}_{6}$ & 607.66 & 612.50 & 1.05 \\
\hline S7 & $\mathbf{C}_{7}$ & 720.76 & 724.95 & 0.67 \\
\hline s9 & $C_{9}$ & 894.91 & 900.16 & 0.58 \\
\hline S10 & $c_{10}$ & 981.99 & 987.95 & 0.71 \\
\hline S13 & $c_{13}$ & 1284.33 & 1293.91 & 0.50 \\
\hline S14 & $c_{14}$ & 1371.40 & 1381.42 & 0.43 \\
\hline S15 & $C_{15}$ & 1458.48 & 1469.14 & 0.64 \\
\hline $\mathrm{S} 17$ & $C_{17}$ & 1673.74 & 1688.66 & 0.77 \\
\hline S18 & $\mathrm{C}_{18}$ & 1760.82 & 1776.17 & 0.43 \\
\hline S19 & $C_{19}$ & 1847.89 & 1863.71 & 0.46 \\
\hline $\mathrm{S} 20$ & $c_{20}$ & 1934.97 & 1951.83 & 1.04 \\
\hline
\end{tabular}

Table S2. 1 Deuterium uptake values calculation for serine residues in the model peptide.

$\mathrm{m} / \mathrm{z}^{\mathrm{a}}$ recorded for lipid sequence without $\mathrm{D}_{2} \mathrm{O}$

D uptake ${ }^{b}$ calculated for each residue 


\section{Appendix B}

Figure S3. 1. Mass spectrum showing the ions obtained upon ESI of the Nt17-POPC and Nt17POPE vesicle systems (compare with Figure 1 in the manuscript). For labeling purposes, a large red circle represents a single Nt17 peptide, a green triangle represents a single lipid molecule. Insets show expanded regions in which specific ions would be present. Notably the Nt17+7POPC, Nt17+6POPE and Nt17+7POPE ion species are not observed although their counterparts for the DOPC system are present in relatively high abundance (Table 1 in the manuscript).

Figure S3. 2. Relative intensities of lipid-bound peptide peaks in mass spectra for Nt17-DOPC (A) Nt17-POPC (B) and Nt17-POPE (C) systems. In each case $\left[\mathrm{Nt} 17+2 \mathrm{H}^{2+}\right.$ has been chosen as the reference for scaling (ratioing) the intensities. Lipids bound to monomer, dimer, trimer and tetramer are shown in blue, orange, yellow and gray, respectively.

Figure S3. 3. Isolation of $[\mathrm{Nt} 17+1 \mathrm{~L}+2 \mathrm{H}]^{2+}$ ions for DOPC $(\mathrm{A})$ and POPE $(\mathrm{B})$ in the linear ion trap mass spectrometer. For labeling purposes, a large red circle represents a single Nt17 peptide and a green triangle represents a single lipid molecule.

Figure S3. 4. The relative degree of peptide helicity as a function of simulation time for the three lipid systems. Here the initial structures for the Nt17 peptide exhibit $\sim 70 \%$ helicity. 
Table S1. Structural properties of the modeled lipid membranes.

\begin{tabular}{ccccc}
\hline Lipid bilayer & $\begin{array}{c}\text { Area per lipid from } \\
\text { Experiment }\left(\AA^{2}\right)\end{array}$ & $\begin{array}{c}\text { Calculated average } \\
\text { area per lipid }\left(\AA^{2}\right)\end{array}$ & $\begin{array}{c}\text { Calculated } \\
\text { Thickness }(\mathrm{nm})\end{array}$ & $\begin{array}{c}\text { Calculated radius } \\
\text { of gyration }\end{array}$ \\
\hline DOPC & $67.4^{53}$ & $68.59 \pm 1.02$ & $38.63 \pm 0.03$ & $27.42 \pm 0.01$ \\
POPC & $64.3^{54}$ & $65.12 \pm 1.01$ & $39.03 \pm 0.15$ & $26.91 \pm 0.03$ \\
& & & \\
POPE & $56.6^{55}$ & $57.26 \pm 1.01$ & $42.27 \pm 0.45$ & $25.75 \pm 0.16$ \\
\hline
\end{tabular}

Table S3. 1 Structural properties of the modeled lipid membranes. 

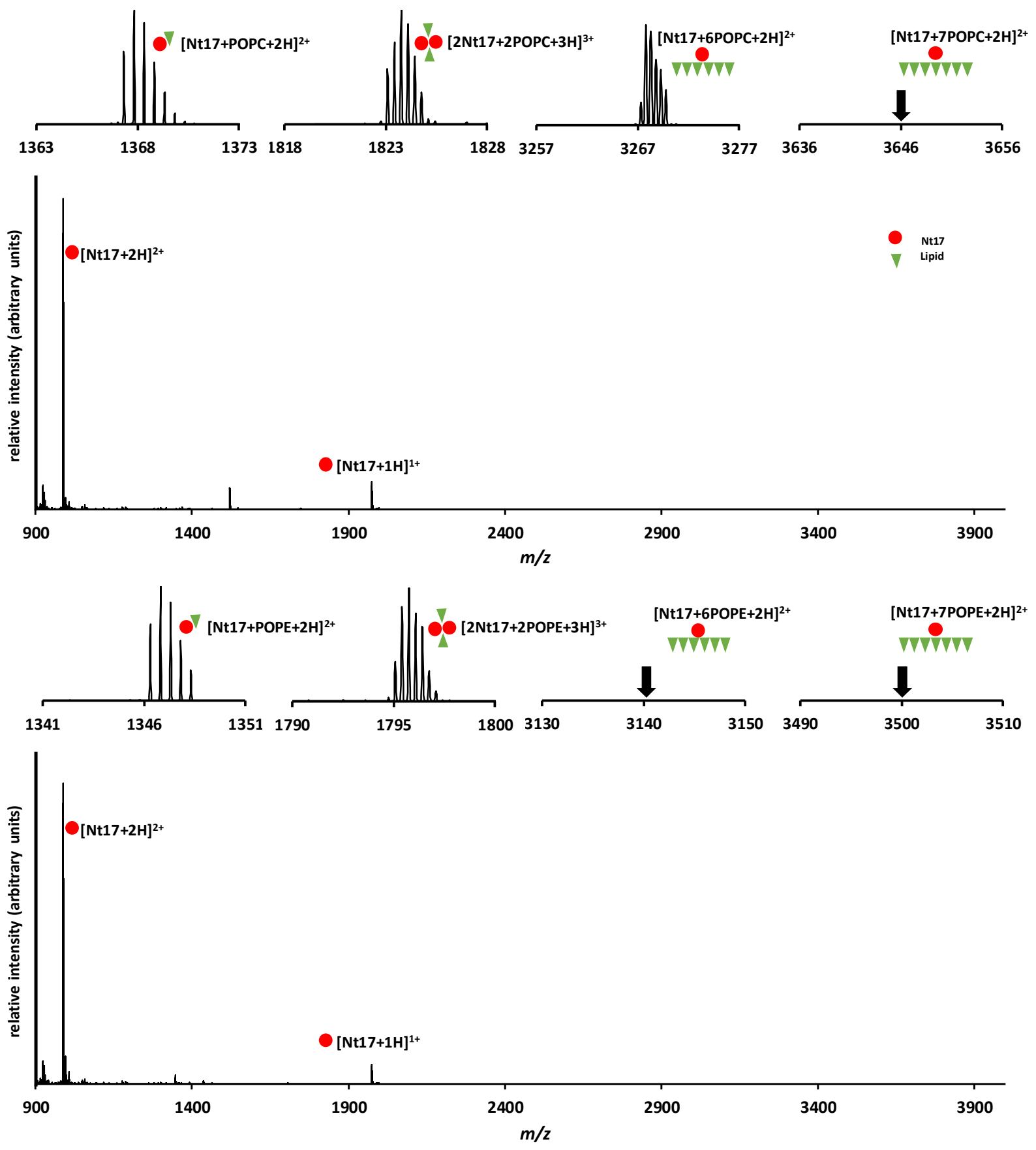

Figure S3. 1 Mass spectrum showing the ions obtained upon ESI of the Nt17-POPC and Nt17POPE vesicle systems.

Reproduced with permission from the Journal of Mass Spectrometry: Investigating the interactions of the first 17 amino acid residues of Huntingtin with lipid vesicles using mass spectrometry and molecular dynamics. Kiani Karanji, A., Beasley, M., Sharif, D., Ranjbaran A., Legleiter, J. and Valentine, S. J. Journal of Mass Spectrometry 55 (1), e4470. Copyright (C 2019 John Wiley \& Sons, Ltd. 


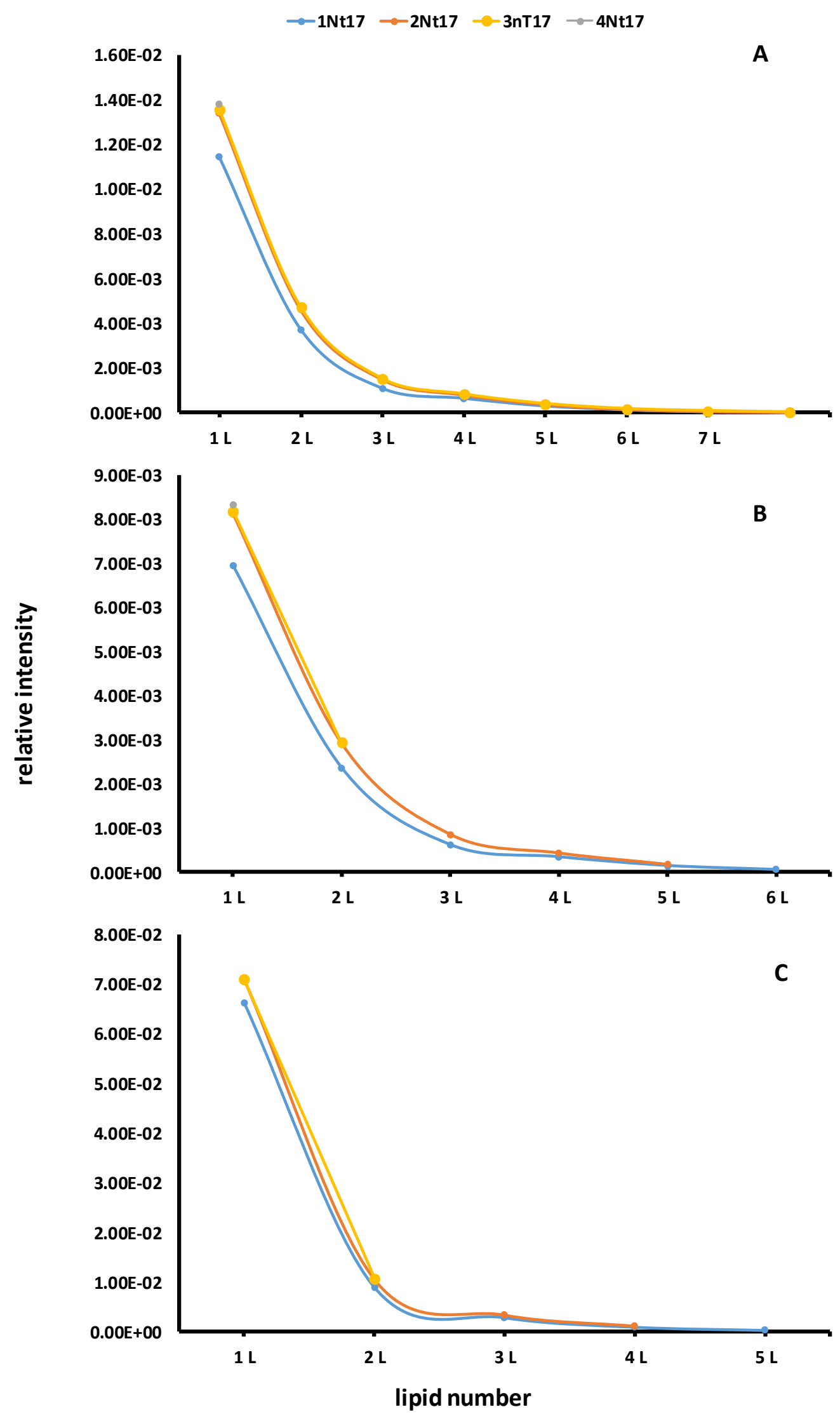


Figure S3. 2 Relative intensities of lipid-bound peptide peaks in mass spectra for Nt17-lipid vesicles.

Reproduced with permission from the Journal of Mass Spectrometry: Investigating the interactions of the first 17 amino acid residues of Huntingtin with lipid vesicles using mass spectrometry and molecular dynamics. Kiani Karanji, A., Beasley, M., Sharif, D., Ranjbaran A., Legleiter, J. and Valentine, S. J. Journal of Mass Spectrometry 55 (1), e4470. Copyright (c 2019 John Wiley \& Sons, Ltd.

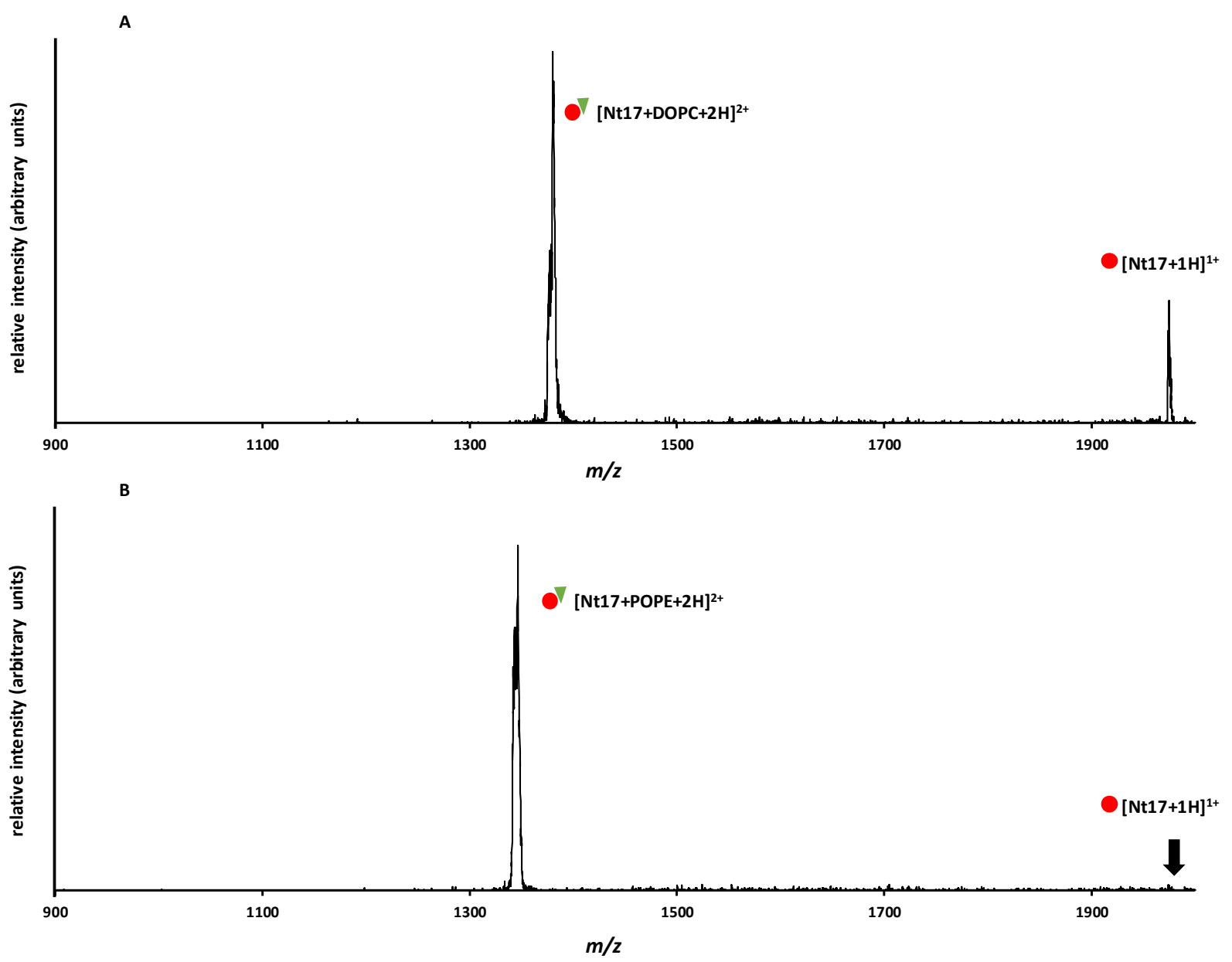

Figure S3. 3 Isolation of $[\mathrm{Nt} 17+1 \mathrm{~L}+2 \mathrm{H}] 2+$ ions for DOPC $(\mathrm{A})$ and POPE $(\mathrm{B})$ in the linear ion trap mass spectrometer.

Reproduced with permission from the Journal of Mass Spectrometry: Investigating the interactions of the first 17 amino acid residues of Huntingtin with lipid vesicles using mass spectrometry and molecular dynamics. Kiani Karanji, A., Beasley, M., Sharif, D., Ranjbaran A., Legleiter, J. and Valentine, S. J. Journal of Mass Spectrometry 55 (1), e4470. Copyright (C 2019 John Wiley \& Sons, Ltd. 


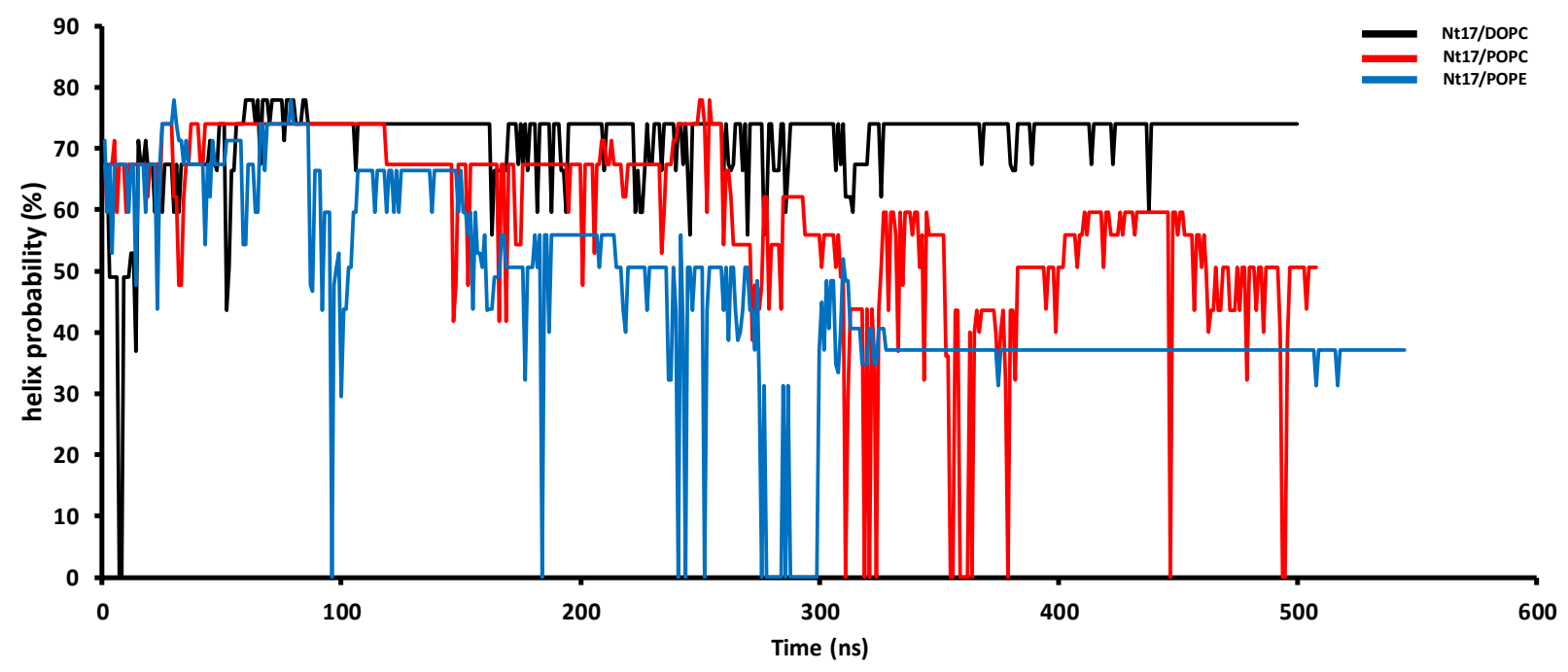

Figure S3. 4 The relative degree of peptide helicity as a function of simulation time for the three lipid systems.

Reproduced with permission from the Journal of Mass Spectrometry: Investigating the interactions of the first 17 amino acid residues of Huntingtin with lipid vesicles using mass spectrometry and molecular dynamics. Kiani Karanji, A., Beasley, M., Sharif, D., Ranjbaran A., Legleiter, J. and Valentine, S. J. Journal of Mass Spectrometry 55 (1), e4470. Copyright (C 2019 John Wiley \& Sons, Ltd. 


\section{Appendix C}
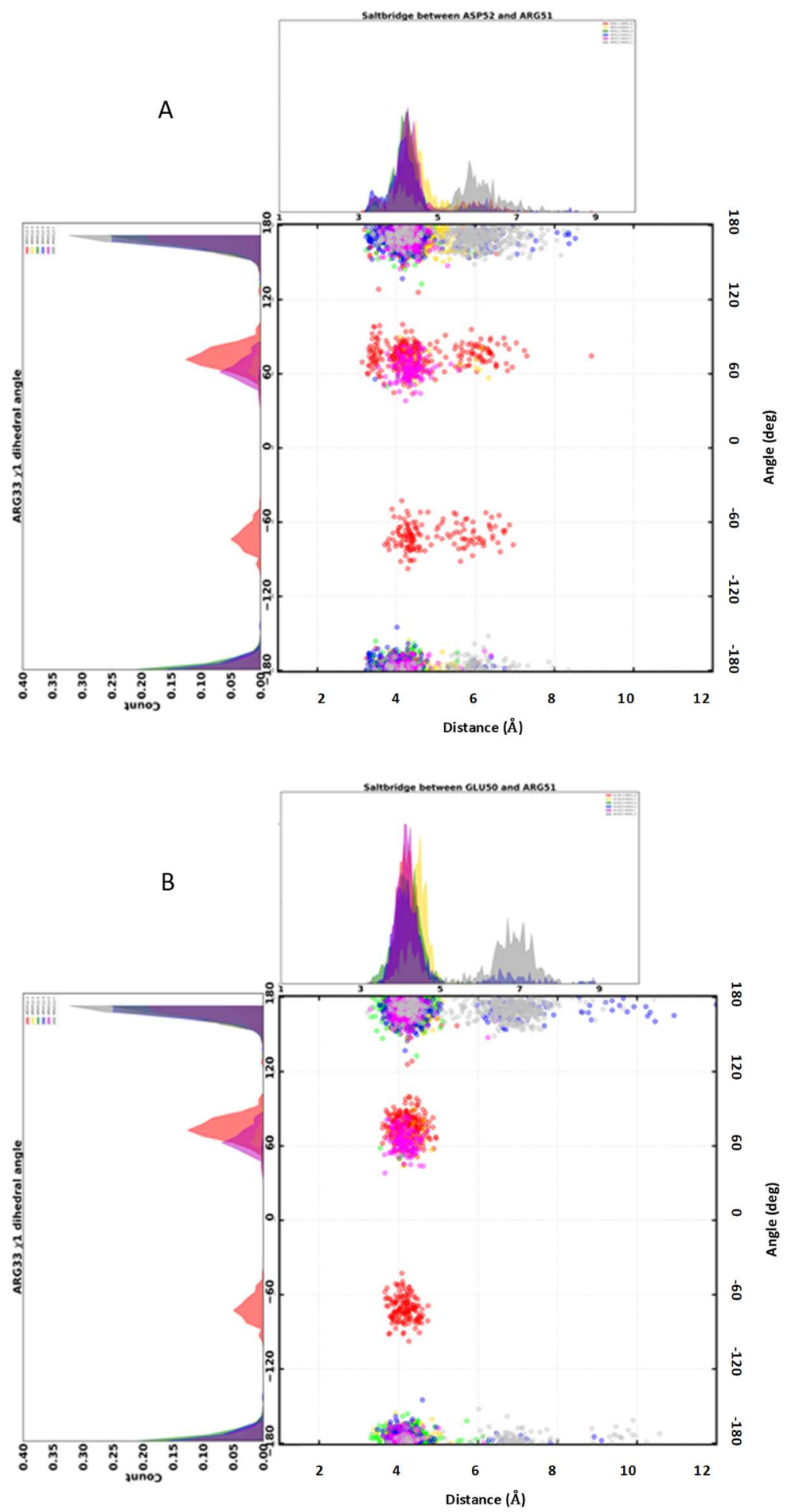

Figure S4. 1 R51 dihedral angle in DHPC-H. A) D52-R51 distance, B) E50-R51 distance. 

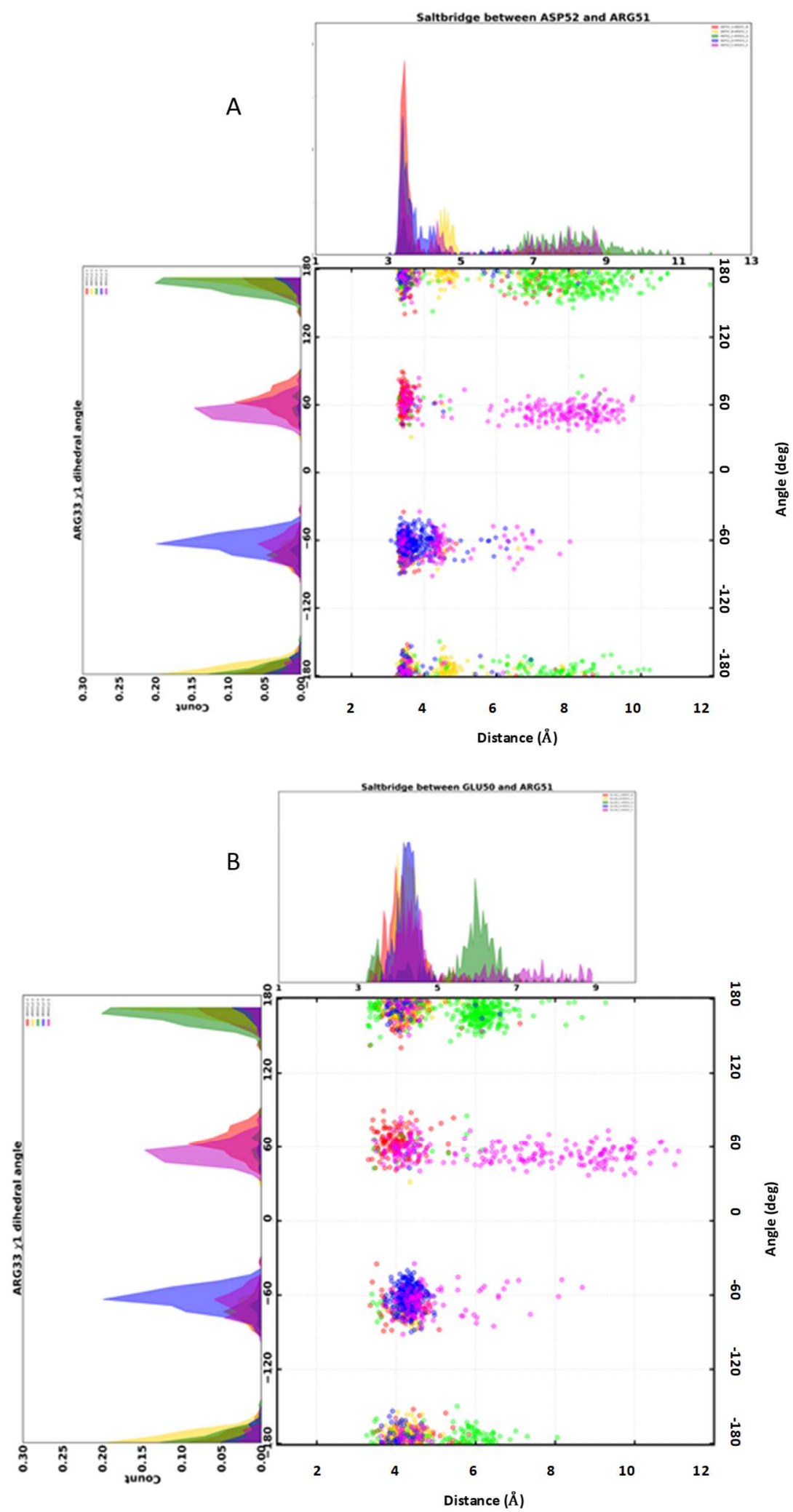

Figure S4. 2 R51 dihedral angle in DHPC-P. A) D52-R51 distance, B) E50-R51 distance. 

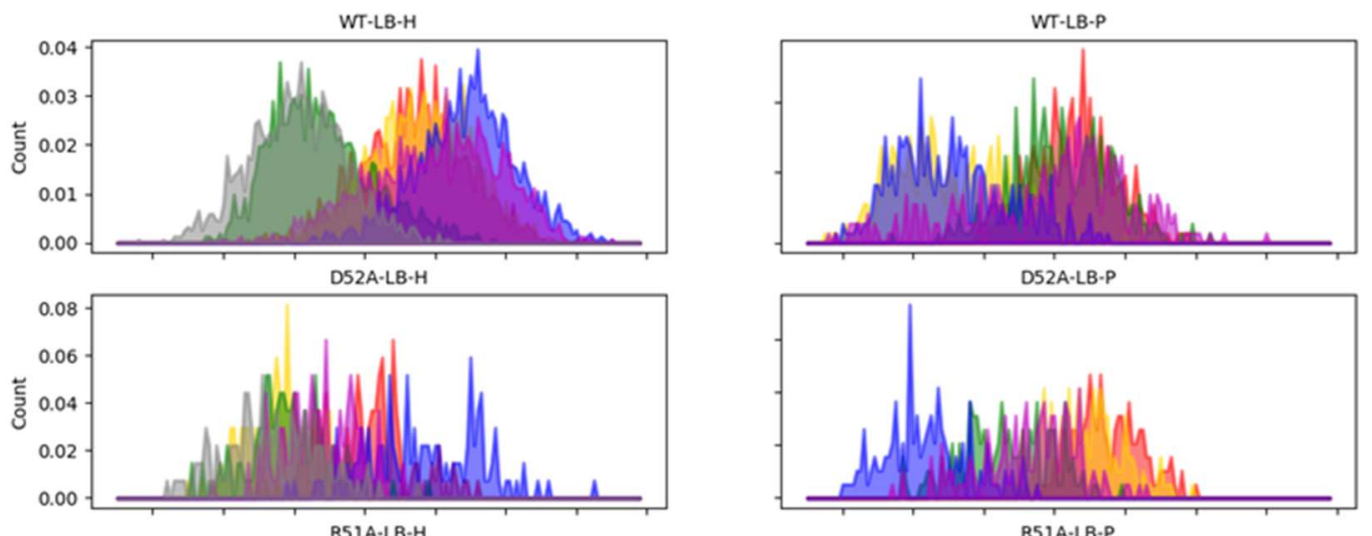

$\square$ A-B

$B-C$

$\square C-D$

$\square$ D-E

RS1A-LB-H

$\square \mathrm{E}-\mathrm{F}$
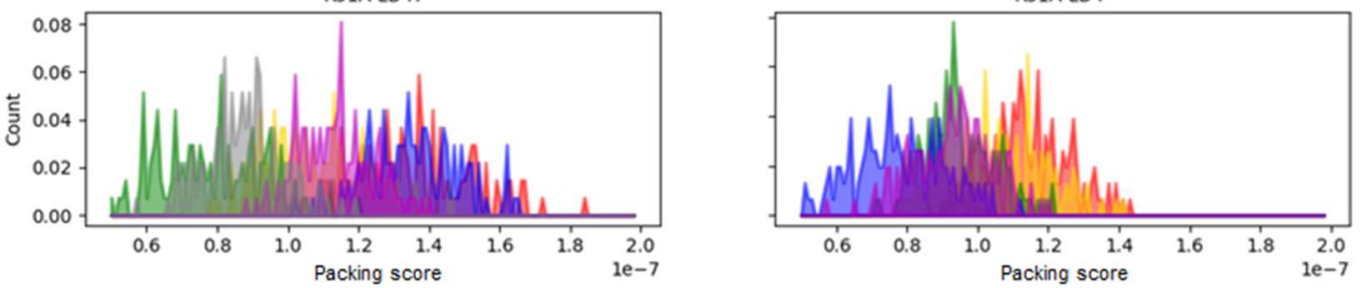

$\square$ F-A

Figure S4. 3 Packing score for WT vs mutations.
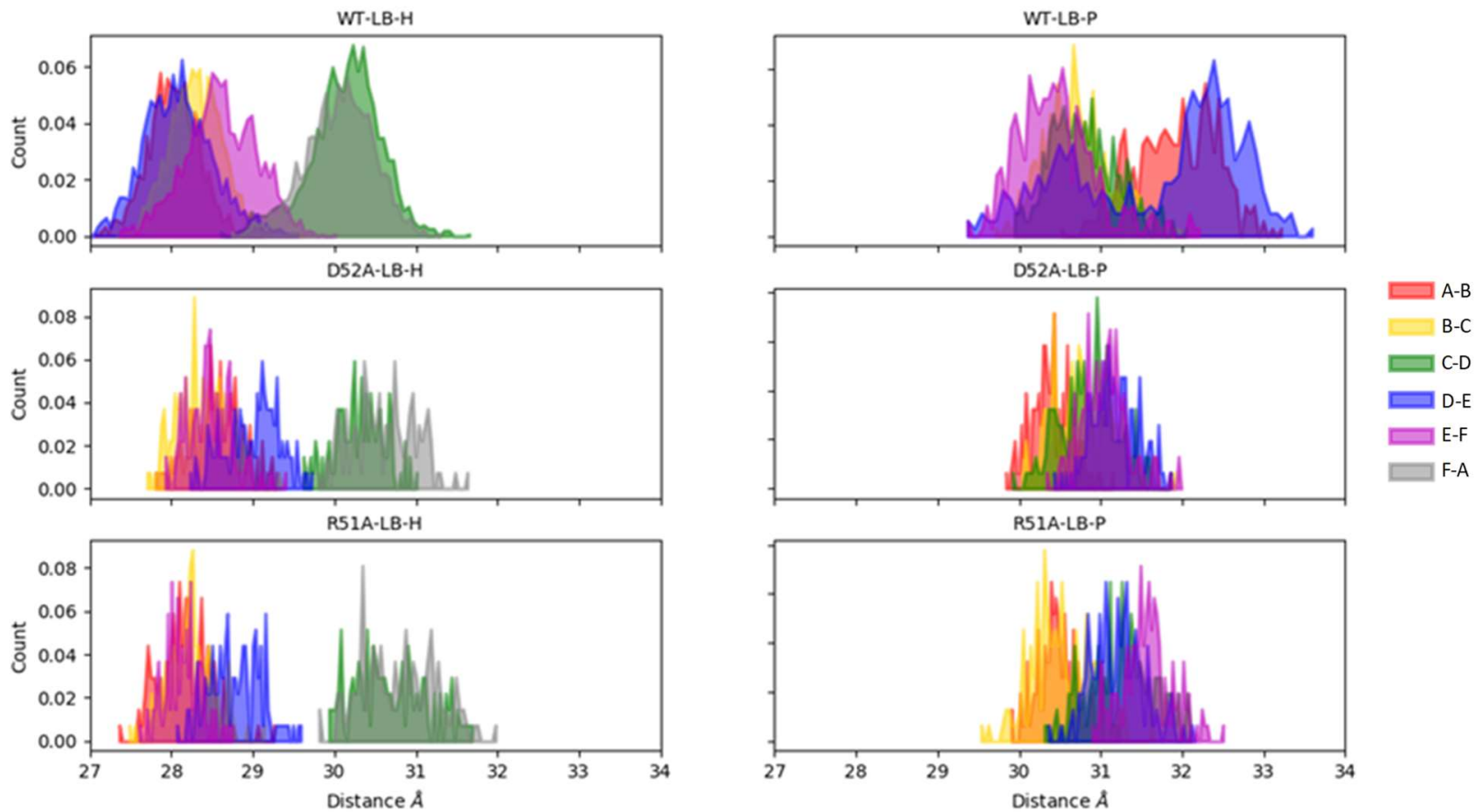

Figure S4. 4 Distance between the center of monomers, WT vs mutations. 

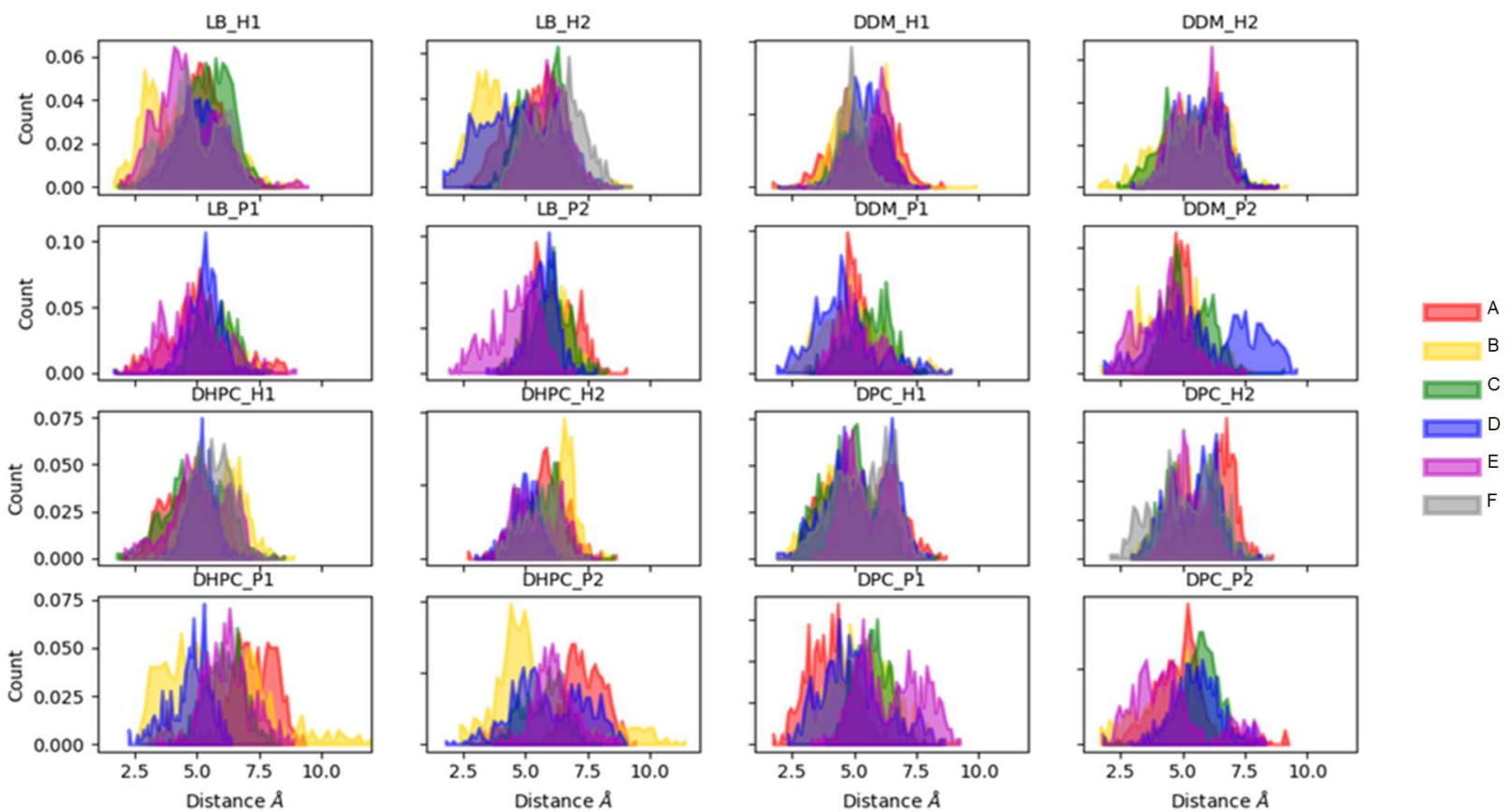

Figure S4. 5 Distance between H75 and D97. 

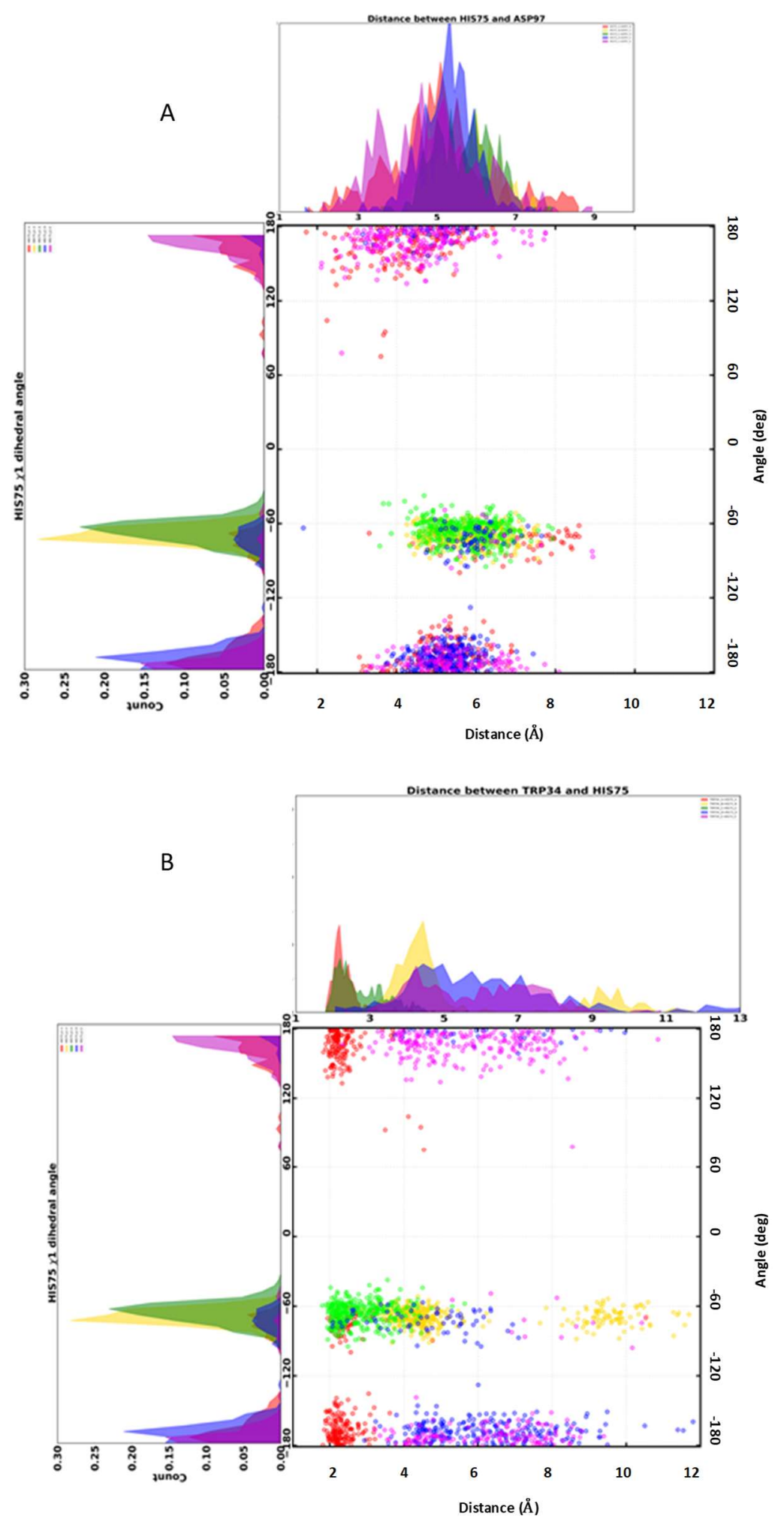

Figure S4. 6 H75 dihedral angle. A) H75-D97 B) W34-H75 distances in LB-Pentamer system. 

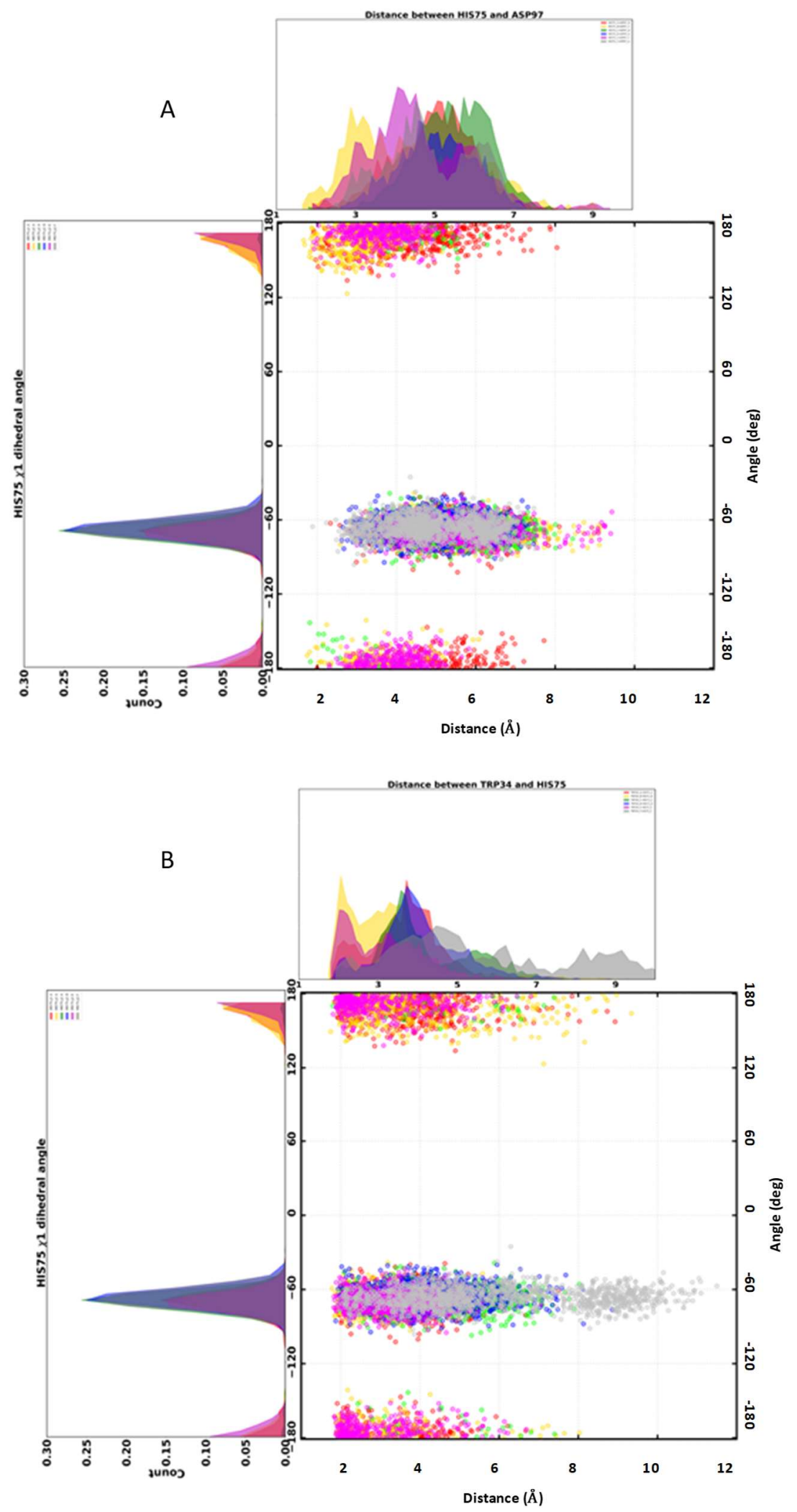

Figure S4. 7 H75 dihedral angle. A) H75-D97 B) W34-H75 distances in LB-Hexamer system. 